\title{
Assessment of Cerebral Blood Flow Changes Following Concussion in Collegiate and High School Athletes
}

\author{
A Dissertation \\ Presented to \\ The Faculty of the Curry School of Education \\ University of Virginia
}

\author{
In Partial Fulfillment \\ of the Requirement for the Degree \\ Doctor of Philosophy
}

by

Shellie Acocello

April 1, 2014 
(C) Copyright by Shellie Acocello

All Rights Reserved May 2014 


\begin{abstract}
Current recommendations for diagnosis and management of sport related concussion include patient-reported symptom inventories, computerized neurocognitive testing, and assessment of balance. There is a dire need for tools that are easily accessible and able to objectively measure physiological, not just functional, changes associated with concussion. The Brain Acoustic Monitor may offer a solution through examination of alterations in arterial functioning post-concussion. In this study, our purpose was to evaluate the potential usefulness of the Brain Acoustic Monitor in detecting changes in cerebral blood flow via measures of arterial stiffness in concussed individuals. In manuscript one, we sought to describe the test-retest reliability of Brain Acoustic Monitor measures and our results indicated moderate reliability over a 24 to 48 hour time period. Very low reliability was seen, however, in one of the eight measures, left systolic interval, suggesting potential sensor malfunction or inconsistency in processing methods. In manuscript two, we sought to describe post-concussion changes in arterial stiffness at 24 to 48 hours post-injury, resolution of symptoms, and return-to-activity. No significant differences in these three time points were found within our concussed sample; however, the concussed group displayed significantly higher right systolic interval at initial assessment and resolution of symptoms compared to a group of healthy age, gender, and sport-matched controls. These findings suggest the need for baseline measurements in future studies as well as a re-examination of processing procedures as there was large variability in some measures. In the third manuscript, we sought to determine how measures of arterial stiffness relate to current standards of concussion assessment,
\end{abstract}


particularly computerized neurocognitive test performance. The results of this study suggested that improvement in arterial stiffness, shown by an increase in left peak interval, resulted in a worsening, or increase, in processing speed performance on the Concussion Resolution Index neurocognitive test battery. While this relationship was not expected, it was observed that the arterial stiffness waveforms of many of the concussed sample displayed an anomalous second reflection. This additional reflection could, in theory, be due to poor or lack of standardized placement of sensors or the result of a wave reflection from secondary sites within the arterial tree. Based on these findings, it seems that the Brain Acoustic Monitor is not a conclusively strong addition to current concussion assessment practices and management of concussion should remain reliant upon a multifactorial approach including symptom inventory, balance assessment, and neurocognitive test performance 


\section{ACKNOWLEDGMENTS}

First and foremost I want to thank my Lord and Savior, Jesus Christ, for giving me life and blessing me with the many opportunities I've had along my educational career. I am nothing without You and all my endeavors would have been in vain without You guiding the way. Thank You, God, for giving me the most important gift of all.

Thank you to Active Signal Technologies, Inc. and Arthur Cooke for the opportunity to work with the Brain Acoustic Monitor. Thank you for your constant availability and guidance throughout the last four years as I've learned more about the device and its applicability to sports concussion. I look forward to a continued relationship in our endeavor to provide an objective measure to the concussion management tool box.

Thank you to my dissertation committee, Joe Hart, Jay Hertel, Donna Broshek, and Susan Saliba, for your encouragement, guidance, and support during my time at UVA, not just in my professional career but in many facets of life. It has been a great honor to learn from the best and I hope to one day have a similar impact on my own students as you've had on me.

I especially need to thank my academic advisor and dissertation chair, Susan Saliba, for her continuous understanding and support as I have developed as a student, educator, and researcher. I could not have gotten through many obstacles without your guidance, patience, and encouragement. You've taught me that the important things in life are what you make them and the rest falls into place. I am so grateful for this perspective and to have had such a wonderful model as a mentor and advisor. I also would like to thank Donna Broshek for her mentorship and encouragement throughout the last four years. I've truly learned so much from you and have enjoyed collaborating on various research studies. I thank you for your patience and overwhelming kindness as we've worked together. You have been a great inspiration to me as I've developed my research interest and I hope to continue to work with you throughout my career.

I would also like to mention fellow doctoral students in the Sports Medicine program. It has been a great journey with many joys and not-so-fun moments and you all were there each step of the way as support, for advice, and just as friends. I am so grateful for the friendships I've gained along the way. I also want to thank my friend Sonia from the "other side" of the school of education. I am so blessed to have met you and found such a great friend both in the academic world and in life. I greatly appreciate the encouragement and advice you have given me the last few years. I'll miss our Starbucks study dates more than you know!

Lastly, but definitely not least, I want to thank my family. Mom and Dad, you have been so supportive and pushed me to do my best in everything I do. This quality has enabled me to do things I never dreamt of and I hope to guide my own kids the way you have---always in love and wanting the best for me. To Mike, you have no idea how much you mean to me. Things have definitely not been easy over the last two years and you have always stepped up to take care of me and Kynleigh so that I could focus on finishing my education. I could not have done it without you. 
TABLE OF CONTENTS

\section{SECTION I: FRONT MATTER}

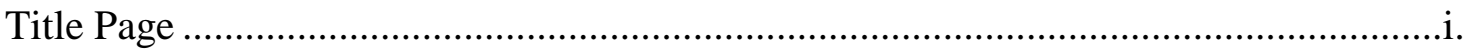

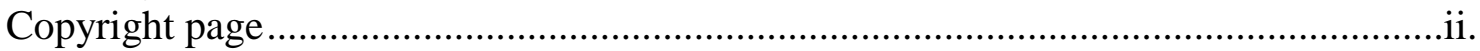

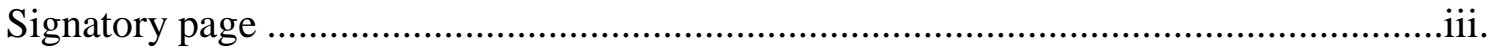

Acknowledgements ....................................................................................................iv.

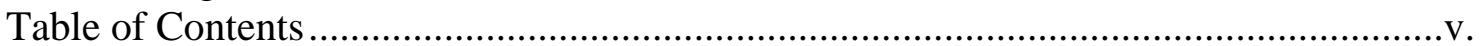

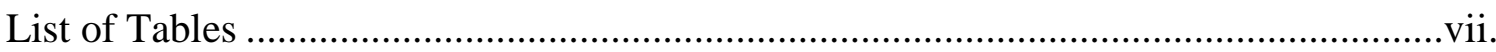

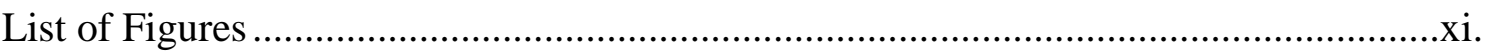

\section{SECTION II: MANUSCRIPTS}

\section{MANUSCRIPT I}

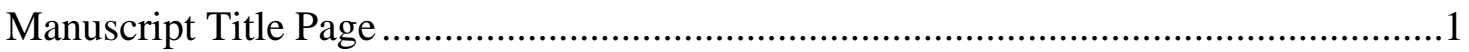

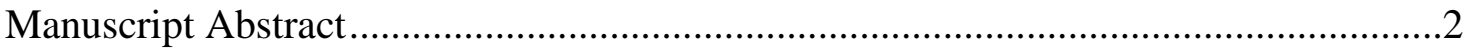

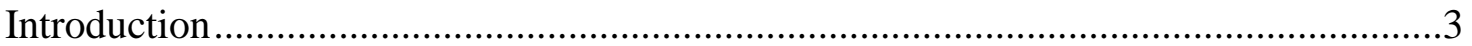

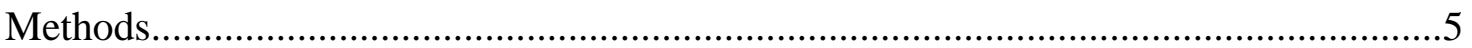

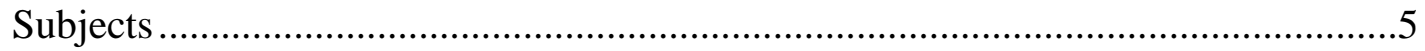

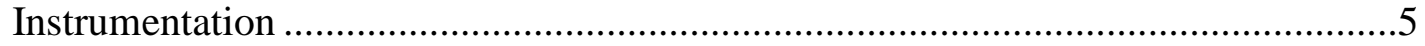

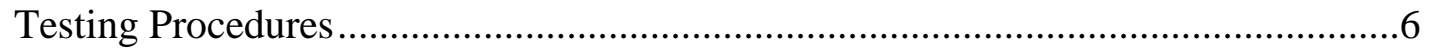

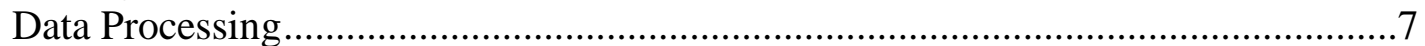

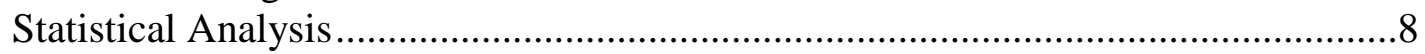

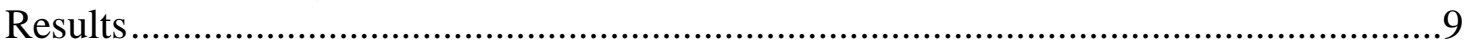

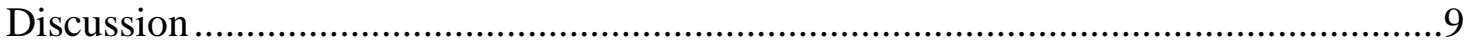

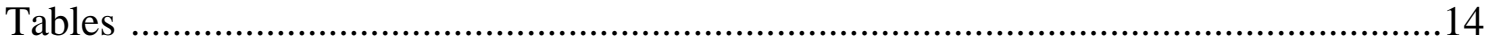

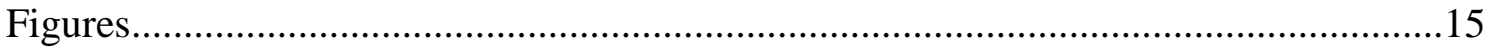

\section{MANUSCRIPT II}

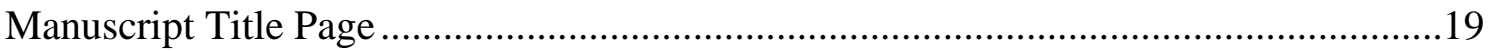

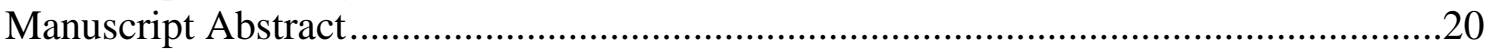

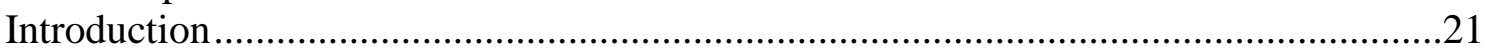

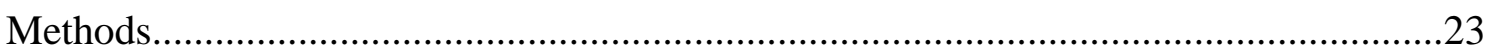

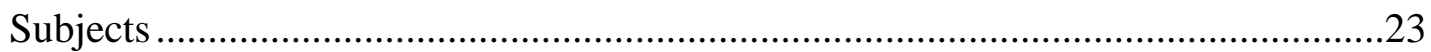

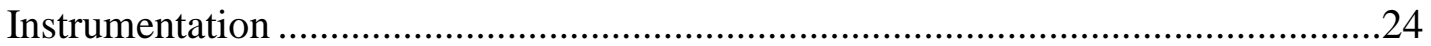

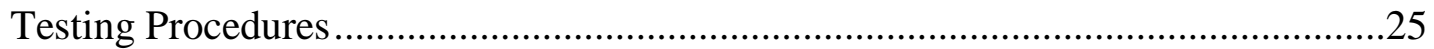

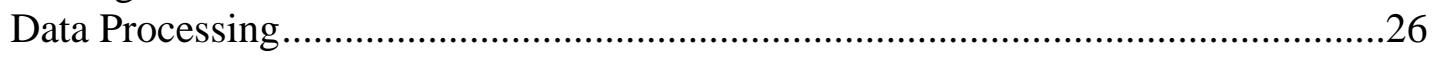

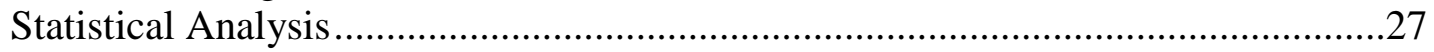

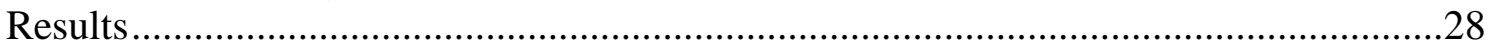

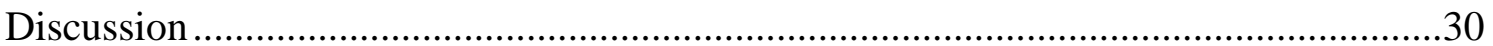

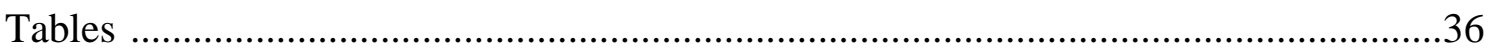

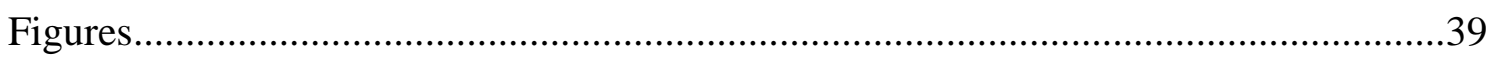




\section{MANUSCRIPT III:}

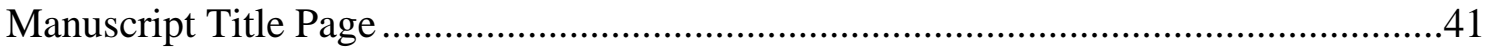

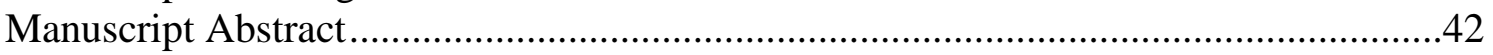

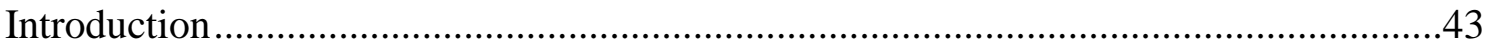

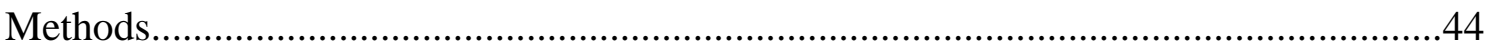

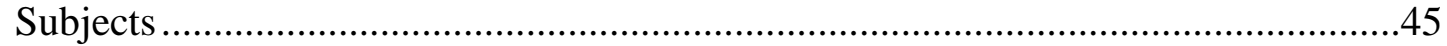

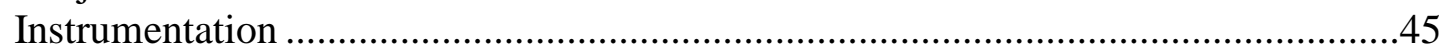

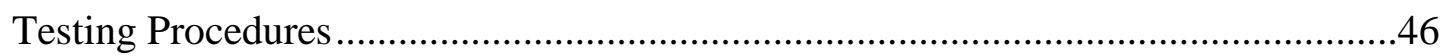

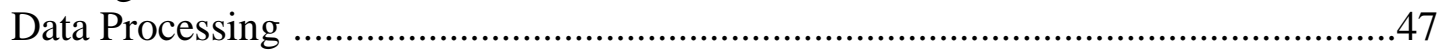

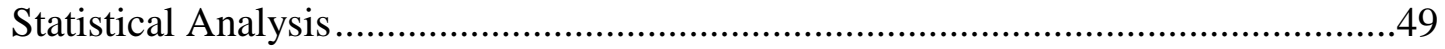

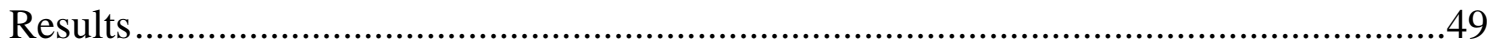

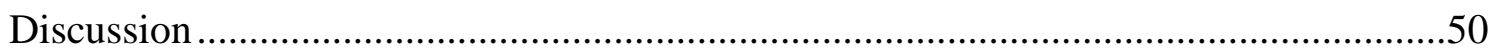

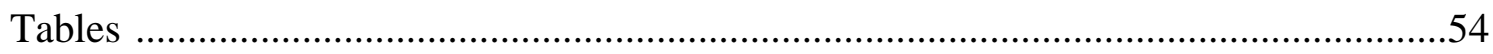

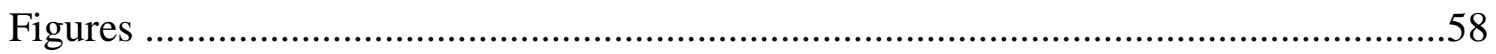

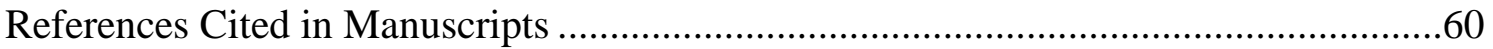

\section{SECTION III: APPENDICES}

\section{APPENDIX A: THE PROBLEM}

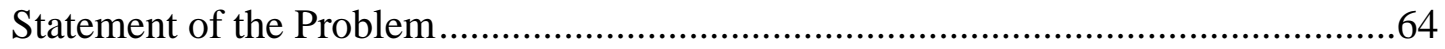

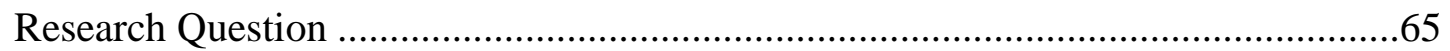

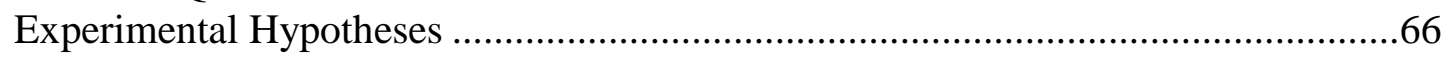

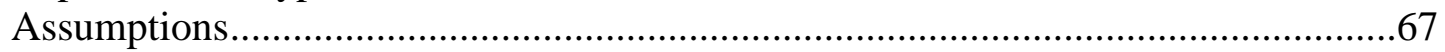

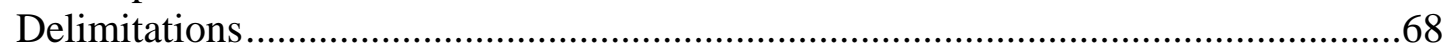

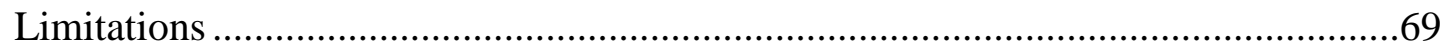

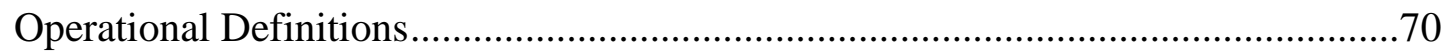

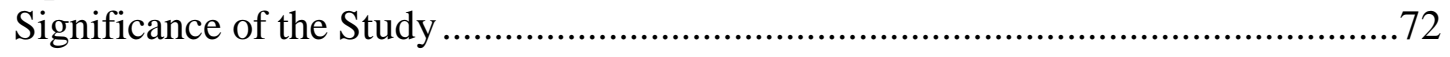

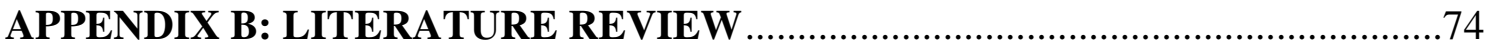

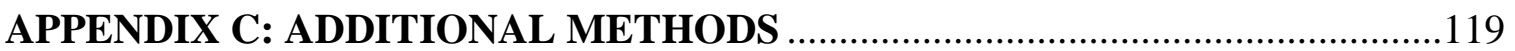

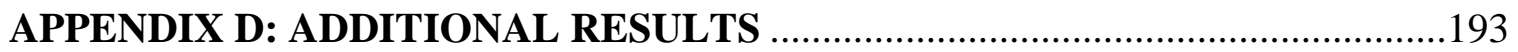

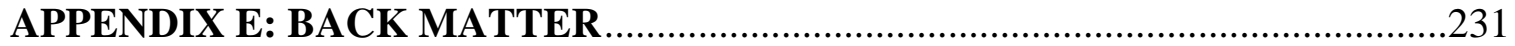

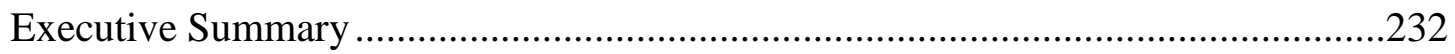

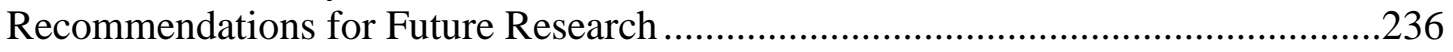

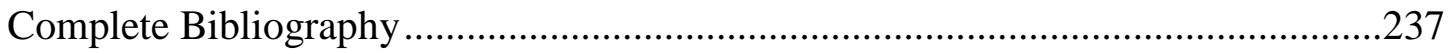




\section{LIST OF TABLES}

Table 1 Test-retest intra-class correlations for left and right BAM variables .14

Table 2 Subject Demographics, Sport Type, and Concussion History for Concussed and Healthy Groups. Time to First Assessment, Symptom Resolution, and Return-to-Play for Concussed Group.

Table 3 Concussed and healthy group scores for Standardized Assessment of Concussion, Modified Balance Error Scoring System, total symptoms, and symptom severity.

Table 4 Concussed and healthy group scores for left and right systolic interval, steepest rise gradient, peak interval, and augmentation index ........................38

Table 5 Subject demographics, sport type, and concussion history for concussed and

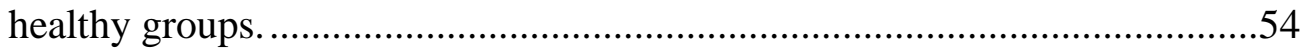

Table 6 Concussed and healthy group means, standard deviations, medians, and interquartile ranges for BAM variables .......................................................55

Table 7 Neurocognitive test scores and SCAT3 outcomes for concussed and healthy

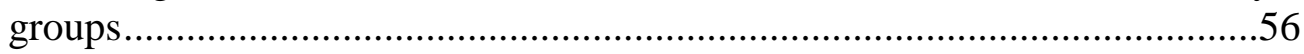

Table 8 Spearman's Rho correlations between neurocognitive assessments and BAM outcomes ...........................................................................................57

Table B1 Frequency of head impacts sustained by position in 2005 and 2006 football

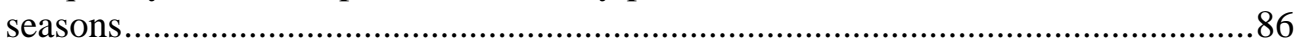

Table C1 University of Virginia Institutional Review Board Approved Protocol (\#16857)

Table C2 University of Virginia Institutional Review Board Approved Consent Form (\#16857) ... .153

Table C3 University of Virginia Institutional Review Board Approved Assent Form ............162

Table C4 Patient Medical History Form for Reliability Study (Manuscript I) ........................164

Table C5 Exercise History Questionnaire for Reliability Study (Manuscript I) ………….......166

Table C6 Data Collection Sheet for Reliability Study ……………………………………....168

Table C7 Data Collection Sheet for Concussion Study …………………………………....170

Table C8 Sideline Concussion Assessment Tool, version 3(SCAT3) …………………….......171

Table C9 Process for creating patient file on BAM ...........................................................175

Table C10 Process for obtaining BAM readings ...................................................................177

Table C11 SCAT3 Testing Procedures …………………………………………….......180

Table C12 Administration of the Concussion Resolution Index................................................182

Table C13 Data processing procedures for BAM data ……………………………………....185

Table C14 Extraction of CRI indices ..............................................................................191

Table D1 Descriptive statistics for subject demographics and gender frequency .....................193

Table D2 Descriptive statistics for left and right systolic interval, steepest rise gradient, peak interval, and augmentation index for Day 1 and Day 2 measurements ...........193

Table D3 Wilcoxon Signed-Rank Test comparing Day 1 and Day 2 measures of left systolic interval, steepest rise gradient, peak interval, and augmentation index .......194

Table D4 Intraclass Correlation Coefficient for Day 1 and Day 2 left systolic interval ...........195

Table D5 Intraclass Correlation Coefficient for Day 1 and Day 2 left steepest rise gradient..195

Table D6 Intraclass Correlation Coefficient for Day 1 and Day 2 left peak interval. 
Table D7 Intraclass Correlation Coefficient for Day 1 and Day 2 left augmentation index ....196

Table D8 Intraclass Correlation Coefficient for Day 1 and Day 2 right systolic interval ........196

Table D9 Intraclass Correlation Coefficient for Day 1 and Day 2 right systolic interval ........196

Table D10 Intraclass Correlation Coefficient for Day 1 and Day 2 right systolic interval ........196

Table D11 Intraclass Correlation Coefficient for Day 1 and Day 2 right systolic interval........197

Table D12 Spearman's Rho Correlations between Day 1 and Day 2 measures for Left BAM variables

Table D13 Spearman's Rho Correlations between Day 1 and Day 2 measures for Right BAM

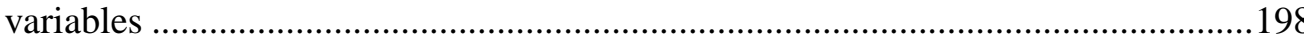

Table D14 Descriptive statistics for subject demographics, sport type, history of previous concussion, and number of previous concussion for Concussed Group ..................198

Table D15 Distribution (frequency) of genders, sport type, and number of previous concussion among Concussed Group....

Table D16 Descriptive statistics and tests of normality for Concussed Group (n=17) SAC, BESS, Total Symptoms, and Symptom Severity at 24-48 hours post-injury, Symptom resolution, and Return-to-Play .200

Table D17 Descriptive statistics for Concussed Group $(n=17)$ time of first assessment, tim to symptom resolution, and time to return-to-play .200

Table D18 Descriptive statistics for Concussed Group ( $n=17)$ Augmentation Index, Peak Interval, Systolic Interval, and Steepest Rise Gradient at 24-48 hours post-injury, Symptom resolution, and Return-to-Play. 201

Table D19 Friedman's Rank Test - Left Systolic Interval (Concussed Group) differences at 24-48 hrs post-injury, symptom resolution, and return-to-activity..... .201

Table D20 Friedman's Rank Test - Left Steepest Rise Gradient (Concussed Group) differences at $24-48 \mathrm{hrs}$ post-injury, symptom resolution, and return-to-activity ....202

Table D21 Friedman's Rank Test - Left Peak Interval (Concussed Group) differences at 24-48 hrs post-injury, symptom resolution, and return-to-activity. .202

Table D22 Friedman's Rank Test - Left Augmentation Index (Concussed Group) differences at 24-48 hrs post-injury, symptom resolution, and return-to-activity....202

Table D23 Friedman's Rank Test - Right Systolic Interval (Concussed Group) differences at 24-48 hrs post-injury, symptom resolution, and return-to-activity..... .202

Table D24 Friedman's Rank Test - Right Steepest Rise Gradient (Concussed Group) differences at $24-48 \mathrm{hrs}$ post-injury, symptom resolution, and return-to-activity ....203

Table D25 Friedman's Rank Test - Right Pulse Interval (Concussed Group) differences at 24-48 hrs post-injury, symptom resolution, and return-to-activity....

Table D26 Friedman's Ranke Test - Right Augmentation Index (Concussed Group) differences at $24-48 \mathrm{hrs}$ post-injury, symptom resolution, and return-to-activity ....203

Table D27 Friedman's Rank Test - Standardized Assessment of Concussion (Concussed Group) differences at 24-48 hrs post-injury, symptom resolution, and return-to-activity

Table D28 Wilkcoxon Signed-Rank Test for Related Samples - Standardized Assessment of Concussion (Concussed Group) differences at 24-48 hrs post-injury, symptom resolution, and return-to-activity

Table D29 Friedman's Rank Test - Modified Balance Error Scoring System (Concussed Group) differences at 24-48 hrs post-injury, symptom resolution, and return-toactivity

Table D30 Friedman's Rank Test - Total Symptom (Concussed Group) differences at 24-48 hrs post-injury, symptom resolution, and return-to-activity

viii 
Table D31 Wilkcoxon Signed-Rank Test for Related Samples - Total Symptom (Concussed Group) differences at 24-48 hrs post-injury, symptom resolution, and return-to-activity...

Table D32 Friedman's Rank Test - Symptom Severity (Concussed Group) differences at 24-48 hrs post-injury, symptom resolution, and return-to-activity..... .205

Table D33 Wilcoxon Signed-Rank Test for Related Samples - Total Symptom (Concussed Group) differences at 24-48 hrs post-injury, symptom resolution, and return-toactivity .206

Table D34 Spearman's Rho Correlations between Time to Resolution of Symptoms and Left and Right Systolic Interval, Steepest Rise Gradient, Peak Interval, and Augmentation Index

Table D35 Spearman's Rho Correlations between Time to Return to Play and Left and Right Systolic Interval, Steepest Rise Gradient, Peak Interval, and Augmentation Index

Table D36 Descriptive statistics for subject demographics, sport type, concussion history, and number of prior concussions for Concussed and Healthy Groups.....

Table D37 Distribution (frequency) of genders, sport type, and number of previous concussion among Concussed Group. .209

Table D38 Descriptive statistics for Left Systolic Interval, Steepest Rise Gradient, Peak Interval, and Augmentation Index for Concussed Group at 24-48 hours post-injury, symptom resolution, and Return-to-Play and the Healthy group at baseline

Table D39 Mann-Whitney U Test - Left Systolic Interval differences between Concussed and Healthy Groups

Table D40 Mann-Whitney U Test - Left Steepest Rise Gradient differences between Concussed and Healthy Groups

Table D41 Mann-Whitney U Test - Left Peak Interval differences between Concussed and

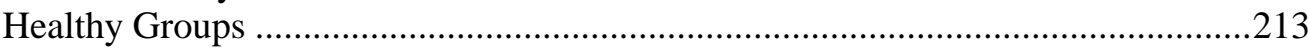

Table D42 Mann-Whitney U Test - Left Augmentation Index differences between Concussed and Healthy Groups

Table D43 Descriptive statistics for Right Systolic Interval for Concussed Group at 24-48 hours post-injury, symptom resolution, and Return-to-Play and Healthy group at baseline

Table D44 Mann-Whitney U Test - Right Systolic Interval differences between Concussed and Healthy Groups.

Table D45 Mann-Whitney U Test - Right Steepest Rise Gradient differences between Concussed and Healthy Groups

Table D46 Mann-Whitney U Test - Right Peak Interval differences between Concussed and Healthy Groups

Table D47 Mann-Whitney U Test - Right Augmentation Index differences between Concussed and Healthy Groups

Table D48 Descriptive statistics for Standardized Assessment of Concussion, Modified Balance Error Scoring System, Total Symptoms, and Symptom Severity for Concussed Group at 24-48 hours post-injury, symptom resolution, and Returnto-Play and for Healthy group at baseline

Table D49 Mann-Whitney U Test - Standardized Assessment of Concussion differences between Concussed and Healthy Groups 
Table D50 Mann-Whitney U Test - Modified Balance Error Scoring System differences between Concussed and Healthy Groups

Table D51 Mann-Whitney U Test - Total symptom differences between Concussed and Healthy Groups

Table D52 Mann-Whitney U Test - Symptom Severity differences between Concussed and Healthy Groups

Table D53 Descriptive statistics for subject demographics, sport type, and concussion history for concussed and healthy groups

Table D54 Distribution (frequency) of genders, sport type, and number of previous concussion among Concussed Group

Table D55 Distribution (frequency) of genders, sport type, and number of previous concussion among Healthy Group ....

Table D56 Descriptive statistics for Right and Left Systolic Interval, Steepest Rise Gradient, Peak Interval, and Augmentation Index for Concussed and Healthy Groups

Table D57 Mann-Whitney U Test - Comparison of Systolic Interval, Steepest Rise Gradient, Peak Interval, and Augmentation Index between Concussed and Healthy Groups.

Table D58 Descriptive statistics for concussed and healthy group processing speed, complex reaction time, and simple reaction time.

Table D59 Descriptive statistics for concussed and healthy groups for SAC, BESS, total symptoms, and symptom severity

Table D60 Mann-Whitney U Test of Independent Means-Comparison of healthy and concussed group processing speed, complex reaction time, and simple reaction time .226

Table D61 Mann-Whitney U Test of Independent Means-Comparison of healthy and concussed group SAC, BESS, Total Symptoms, and Symptom Severity ................227

Table D62 Spearman Rho correlations between CRI and BAM variables ................................228

Table D63 Spearman Rho correlations between SAC, BESS, Total Symptoms, and Symptom Severity and BAM variables

Table D64 Step-wise Linear Regression Model - Prediction of Processing Speed by left and right augmentation index and peak interval

Table D65 Descriptive statistics for time to assessment for concussed group 


\section{LIST OF FIGURES}

Figure 1 BAM laptop, analog-to-digital convertor and sensors ...................................15

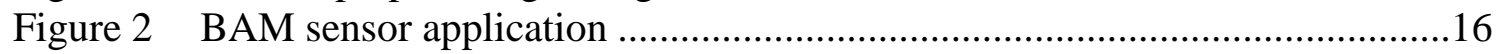

Figure 3 Example arterial pressure waveform ……….............................................16

Figure 4 Comparison of radial arterial pressure wave and BAM forehead signals .....17

Figure 5 Individual subject values for left systolic interval on Day 1 and Day 2 ........18

Figure 6 BAM laptop, signal processing box, and sensors ..........................................39

Figure 7 Example arterial pressure waveform .......................................................39

Figure 8 Example waveforms with abnormal wave reflections ...................................40

Figure 9 BAM sensors applied to forehead and thumb..............................................58

Figure 10 Example arterial pressure waveform …………........................................59

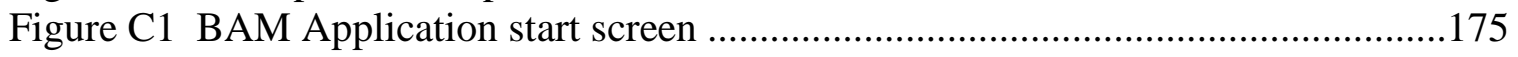

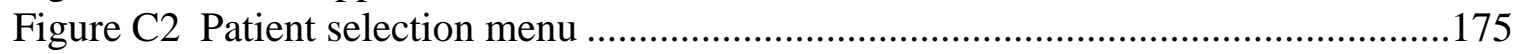

Figure C3 Screen for entering patient identifier ........................................................176

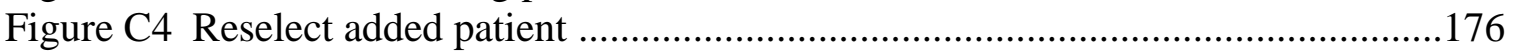

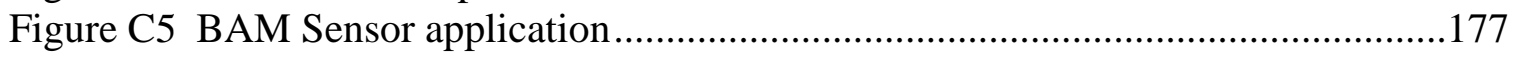

Figure C6 Patient position for BAM readings ………..............................................177

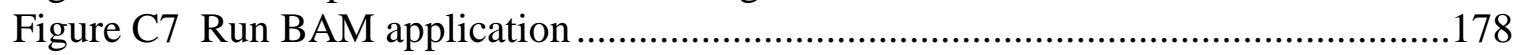

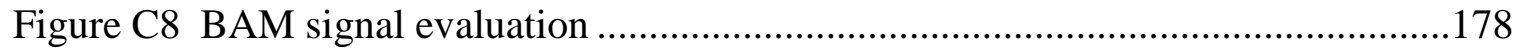

Figure C9 Choose "Analyze" or "Reject Data" ..........................................................179

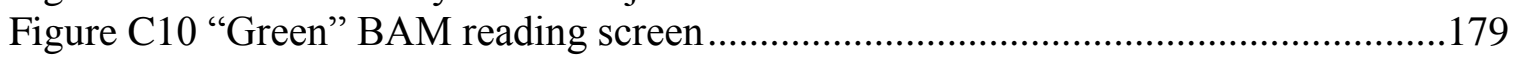

Figure C11 "Red" BAM reading screen ..................................................................180

Figure C12 Select "Tests" to search for test takers..........................................................182

Figure C13 Search profiles and select desired test taker ..............................................183

Figure C14 Set-up new baseline .................................................................................183

Figure C15 Select "baseline" icon to start new baseline ..................................................184

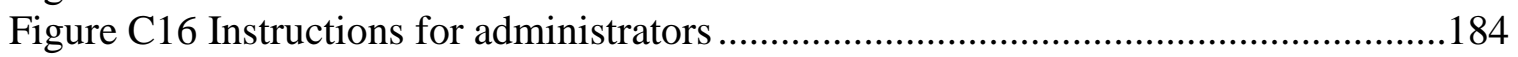

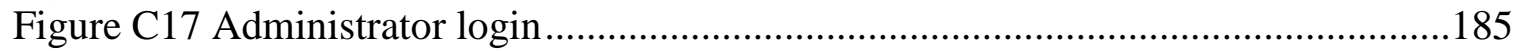

Figure C18 Validation of examination ……………............................................185

Figure C19 Location of "My Documents" folder ........................................................186

Figure C20 Patient file selection for export.................................................................186

Figure C21 Select files with “.STP" extension for export and conversion.......................187

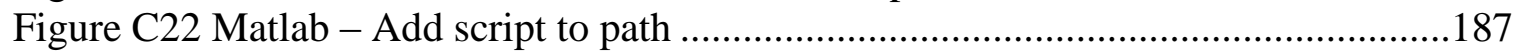

Figure C23 Matlab - Select "BAM.m” script..............................................................188

Figure C24 Enter desired output file name ................................................................188

Figure C25 Select file selection method .................................................................189

Figure C26 Select processes to run .........................................................................190

Figure C27 Select file containing converted BAM files...............................................190

Figure C28 Run BAM application .............................................................................191

Figure C29 Select desired test to create report ............................................................191

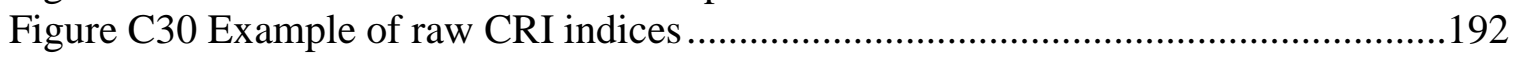


Section II

Manuscript I

Test-Retest Reliability of Brain Acoustic Monitor Outcomes 


\begin{abstract}
Context: A device called the Brain Acoustic Monitor (BAM) has been developed to detect cerebral blood flow changes resulting from mild and severe traumatic brain injury by amplification and comparison of sound waves created by cerebral arteries and a reference artery. Data processing techniques have been developed that allow, via transfer function, creation of an arterial pressure waveform. Prior measures have shown moderate to good reliability; however, newer outcomes have not been assessed. It is necessary to establish their reliability prior to initiating studies using serial assessment. Objective: To determine the test-retest reliability of arterial stiffness measures obtained by the BAM. Design: repeated measures Setting: Laboratory Patients or other participants: 19 healthy, recreationally active individuals (11 females, 8 males; age: $24.2 \pm 3.9$ years; height: $172.9 \pm 8.4 \mathrm{~cm}$; mass: $74.6 \pm 13.8 \mathrm{~kg}$ ). Intervention(s): All subjects were assessed with the BAM at 2 time points separated by at least 24 hours. Main outcome measures: Arterial pressure waveforms were created retrospectively for each BAM reading. Dependent variables calculated using these waveforms included systolic interval, steepest rise gradient, peak interval, and augmentation index. Three waves were selected and averaged for all analyses. Due to the non-normal distribution of our data, Wilcoxon Signed-Rank tests for independent means were used to compare differences between Day 1 and Day 2 measures. Intra-class correlation coefficients (ICCs), Spearman's Rho correlations, and standard error of measurement were calculated. Significance was set to $\mathrm{p} \leq 0.05$. Results: A significant difference was found in measures of left peak interval between day 1 and day 2 measures [medians: Day $1=0.12$ (IQR: 0.11, 0.15); Day $2=$ 0.13 (IQR: 0.12, 0.15); $\mathrm{Z}=-1.972, \mathrm{p}=0.049$ ]. ICCs ranged from 0.02 [Left systolic interval, Day $1=0.12(\mathrm{IQR}=0.11,0.15)$, Day $2=0.13(\mathrm{IQR}=0.11,0.23)]$ to 0.773 [Right steepest rise gradient, Day 1=3.3 (IQR=1.8, 4.7), Day 2=2.8 $(2.2,3.8)]$, however the majority of the ICCs for BAM outcomes fell between 0.65 [Right systolic interval, Day $1=.13(\mathrm{IQR}=0.10,0.23)$, Day $2=0.17(0.11,0.24)$ ] to 0.74 [Left peak interval, Day $1=0.12(0.11,0.15)$, Day $2=0.13(0.12,0.15)]$, indicating moderate reliability. Spearman's rho correlations were significant for four of the eight outcomes including left augmentation index and right systolic interval, steepest rise gradient, and augmentation index. The rho correlations ranged from 0.041 (left systolic interval) to 0.64 (right augmentation index). Conclusion: Reliability of the BAM measures are poor to moderate, with systolic interval and steepest rise gradient displaying more favorable reliability coefficients. There is a great degree of variability in augmentation index and peak interval measures likely due to the presence of an anomalous second wave reflection. Once better understanding is held concerning the role of each wave reflection within the arterial pressure waveform obtained by the BAM, reliability should be reassessed. These reliability values, excluding that found with left systolic interval, are comparable to current assessment techniques such as computerized neurocognitive testing.
\end{abstract}

\title{
Key Words: concussion; mTBI; mild head injury; assessment
}




\section{INTRODUCTION}

Sports concussions have become a major concern in the realm of sports medicine over the last decade. As many as 3.8 million concussions occur annually ${ }^{1}$, emphasizing the need for effective methods of diagnosis and development of individualized management protocols. While the most frequent methods of concussion assessment have used symptom reporting, pre- and post-injury neuropsychological testing and balance assessment,$^{2-5}$ none provide truly objective measurements of the physiological changes that occur following concussion. It is known that drastic ionic imbalances, reduced cerebral blood flow, and immediate increase in glycolytic activity result in a subsequent mismatch of oxygen supply and demand in the cerebrum. ${ }^{6-9}$ This imbalance then leads to chronic hypoglycolysis and reduced cerebral blood flow days to weeks following injury—well after resolution of symptoms and return of neuropsychological function. ${ }^{6-11}$

The Brain Acoustic Monitor (BAM) is a noninvasive device that detects changes in cerebral arterial function, which is common following both concussion and traumatic brain injury. ${ }^{12-15}$ This portable device detects, amplifies, and displays sound waves that are emitted through predictable deformations in the skull in response to each arterial pulse. The BAM measures and displays the real-time amplitude and frequency distributions of these intracranial pulse signals from either side of the skull and compares them to a reference arterial signal (typically a digital artery). In early studies using this device, outcomes were used that described the erraticism of the real time signal and differences in frequency distribution between each forehead sensor and the reference signal. These studies have shown that the BAM was highly sensitive to patients with 
abnormal findings of CT scan after TBI but the data were not highly specific ${ }^{13}$. In a study of mTBI, 369 patients were assessed using CT, symptom reporting, a BAM assessment, and expert panel adjudication. Over two-thirds of the population was redflagged by the BAM prior to final adjudication of mTBI diagnosis by an expert panel. ${ }^{13}$ These results give some indication that the tool may be useful in concussion diagnosis and further investigation of the device in less severely injured populations is warranted More recently, developments have been made in BAM signal processing methods that allow for calculation of an arterial pressure waveform. Using a custom transformation algorithm, the time-domain signal is converted such that systolic pressure waves and wave reflections can be identified. Using these markers, inferences about arterial stiffness can be made. In particular, by looking at the time interval between systolic waves and wave reflections, pulse wave velocity can be indirectly evaluated. ${ }^{16}$ Additionally, by looking at the magnitude of the wave reflection compared to that of the systolic pressure wave, we can measure how well cerebral arteries are able to attenuate pressure which is, in theory, indicative of their viscoelastic properties. ${ }^{16}$ It would be of interest to examine these newer outcomes in head injured populations as they better describe physiological changes in cerebral arterial function that are likely seen following injury.

Before studies in head injured populations begin, especially those using serial assessment to identify recovery to baseline levels, clinicians need to have an understanding of how BAM outputs change on a daily basis. Therefore, the purpose of 
this study was to examine test-retest reliability associated with the BAM in healthy individuals.

\section{METHODS}

A descriptive laboratory study was performed using repeated measures design. There was a single dependent variable (time) for intra-rater reliability at 2 levels: day 1 and day 2. There were eight dependent variables that included left and right systolic interval, steepest rise gradient, peak interval, and augmentation index.

\section{Subjects}

A total of 19 healthy, recreationally active males and females participated in this study ( 8 males, 11 females; age: $24.1 \pm 3.9$ years, height: $172.9 \pm 8.4 \mathrm{~cm}$, mass: $74.6 \pm$ $13.8 \mathrm{~kg}$ ). Participants were excluded if they had a history of unresolved injury or surgery to the lower extremities, neck or spine, a known history of concussion or other head injury within the previous six months, or a known history of seizures. The university's Human Subjects Research Investigational Review Board approved this study and all participants provided informed consent prior to enrollment.

\section{Instrumentation}

The BAM (Acoustic Signal Technologies, Inc., Baltimore, MD) was used to assess global cerebral perfusion. It consists of two 2-cm circular sensors that are held tightly against the skin of the forehead using an elastic band, connected to a signal conditioning box and laptop computer (Figure 1). The two sensors placed on the skull are termed "right" and "left" and provide broader monitoring throughout the skull. The sensors are not specific to perfusion characteristics of each hemisphere of the brain; they 
permit a stereophonic representation of the acoustical signal from two distinct areas and following retrospective processing via a custom transfer function, an arterial pressure waveform results. A single BAM recording requires 10-20 seconds of monitoring time and less than 10 minutes of operator effort.

\section{Procedures}

Participants reported to the Exercise and Sport Injury Laboratory for two study sessions on separate days. Upon arriving, subjects were asked to complete a general medical history questionnaire to assess eligibility. Following a 5 minute bout of quiet sitting, an elastic strap was secured snugly around the skull so that it would lie just above the participant's eyebrows and below the hairline. The two circular sensors were then applied along the lateral border of the eyebrows by slipping them under the elastic band (Figure 2). Some variation in placement did occur based on differences in participants' skull shape and forehead size as well as the quality of the BAM signal achieved. A third sensor was placed on the participants' left thumb using a finger clip so that a reference arterial signal could be obtained. All sensor application and analysis was performed by the same examiner.

Once sensors were in place, participants were asked to sit quietly with their hands at their sides. The examiner assessed the BAM signals during the first 3 to 5 recordings and adjusted the head and finger sensors as needed to improve the quality of the signal using amplitude values and absence of aberrant noise as criteria. Once satisfied with sensor placement, approximately 5 readings were taken and saved to the BAM computer 
hard drive. The same procedures were followed during the second session, which occurred at least 24 hours following the first (mean: 1.6 days).

\section{Data Processing}

Retrospective processing using a custom transfer function occurred after completion of study procedures. These data were imported into Matlab for calculation of pressure pulse waves. Important points on each waveform, which are described in Figure \#, were used for calculation of 4 outcome variables that included systolic interval, peak interval, steepest rise gradient, and augmentation index. Specifically, the systolic peak (P1), the inflection point or peak of the reflected wave (P2), and systolic onset (SO) were identified. The time that lapsed between systolic onset and the first peak was deemed systolic interval and the time that lapsed between the first peak and the peak of the reflected wave was deemed peak interval. Both of these measures indicate the arteriole wall's ability to attenuate pressure, whether it is the result of the initial systolic contraction of the heart or from the rebounded pressure experienced when blood reaches bifurcations in the arteries. The closer these points are to one another, the higher the pulse wave velocity is within the arteriole. These peaks are described in an example in Figure

3. For steepest rise gradient, the highest slope of the initial peak was calculated using a 10 data point window. This measure is directly related to the systolic interval in that, the higher the slope is between systolic onset and the systolic peak, the higher the rate that pressure is being applied to the arterioles. Lastly, the magnitude of the reflected wave in relation to the systolic peak is represented by augmentation index and the smaller this 
value is, the greater the reflected wave. Equations for each of these outcomes, excluding steepest rise gradient, are as follows:

$$
\begin{gathered}
\text { Systolic Interval }(S I)=\text { Time }_{P 1}-\text { Time }_{S O} \\
\text { Peak Interval }=\text { Time }_{P 2}-\text { Time }_{P 1} \\
\text { Augmentation Index }=\frac{\text { Amplitude }_{P 1}-\text { Amplitude }_{P 2}}{\text { Amplitude }_{P 1}} \times 100
\end{gathered}
$$

Following processing, 3 of the 5 readings taken during each testing session were selected and averaged for use in statistical analysis.

\section{Statistical Analysis}

Using three trials each from day 1 and day 2 testing sessions, means and standard deviations were calculated for all dependent variables. Tests of normality revealed a nonnormal distribution in our data, therefore non-parametric tests were used to compare Day 1 and Day 2 values and for calculation of correlation coefficients. Initially, Wilcoxon Signed-Rank tests for related means were used to compare values obtained by each assessor and by a single assessor on day 1 vs. day 2. Secondly, Spearman's Rho correlations were calculated to assess the strength of the relationship between values obtained by each assessor and between day 1 and day 2 measures. Test-retest reliability was quantified using intra-class correlation coefficients $(2, \mathrm{k})$ and interpreted as follows: less than 0.74 was considered poor; 0.75 to 0.89 , good; and 0.90 to 1.00 , excellent. ${ }^{17}$ Standard error of measurement was also calculated as the standard deviation multiplied by the square root of one minus the correlation coefficient ${ }^{18}$. Significance was set at 
$\mathrm{p} \leq 0.05$ and all statistical procedures were carried out using SPSS (version 20.0; SPSS, Inc., Chicago, IL).

\section{RESULTS}

Table 1 contains means, standard deviations, Intra-class Correlations Coefficients, Spearman's Rho correlations, and standard error of measurement for Day 1 and Day 2 measurements. A significant difference was found in measures of left peak interval between day 1 and day 2 measures [medians: Day $1=0.12$ (interquartile range: 0.11 , 0.15); Day $2=0.13$ (interquartile range: $0.12,0.15$ ); $\mathrm{Z}=-1.972, \mathrm{p}=0.049$ ). Intra-class correlations ranged from 0.02 (Left systolic interval) to 0.773 (Right steepest rise gradient), however the majority of the intra-class correlations for BAM outcomes were between 0.65 to 0.74 , indicating poor to moderate reliability. Spearman's rho correlations were significant for four of the eight outcomes including left augmentation index and right systolic interval, steepest rise gradient, and augmentation index. The rho correlations ranged from 0.041 (left systolic interval) to 0.64 (right augmentation index).

\section{DISCUSSION}

The purpose of this study was to examine day to day variability in BAM measures of arterial stiffness to determine its potential utility in serial assessment of cerebral blood flow. Our findings indicate moderate reliability in half of the BAM outcomes, particularly in systolic interval and steepest rise gradient variables. Peak interval and augmentation index intra-class correlations ranged from 0.48 to 0.74 indicating more variability in those measures. The implications of these findings are that the day-to-day variability, while acceptable in some measures, is too large in others and further 
development of the BAM data processing method is needed. The variables that are of most interest, augmentation index and peak interval, displayed the poorest range of reliability, excluding left systolic interval. While these measures afford the most theoretical knowledge regarding changes in arterial stiffness, it is mandatory that we gain a better understanding before research with this device moves forward into a patient population.

Interestingly, left systolic interval had an unarguably poor reliability with an intraclass correlation coefficient of 0.02 , a spearman's rho correlation of 0.041 , and an associated standard error of measurement that was nearly twice the mean of the Day 1 and Day 2 measures in our group. Upon further inspection of this outcome using a subject-by-subject inspection of our data (Figure 5), it seems that there was no systematic differences present within our sample. There were no outliers that significantly altered the reliability of this finding. It is possible that there was a malfunction in the left forehead sensor, however because left side measures of steepest rise gradient and peak interval were acceptable, this is unlikely. Further, the method by which the raw acoustic signal is spliced into individual pulses during processing used the systolic peak as a marker of alignment rather than systolic onset. This technique likely introduced a larger magnitude of variability in identification of systolic onset, which is integral in calculation of systolic interval.

During data processing, each waveform was inspected post-transformation to assess the quality of the raw signal. During this inspection, study investigators were seeking to identify a larger first peak, i.e. the systolic peak, and a smaller wave reflection. 
In a large number of subjects, it seems that two wave reflections were present and the presence of a second reflection has likely influenced the variability of these measures in our sample. While not every subject had two wave reflections, those that did were consistent between days. This is potentially due to anatomical variations in an individual's cerebrovascular anatomy or in the amount of pressure present in their cerebral arteries. One theory regarding the second reflected wave, which appeared to arrive earlier than the larger reflected wave, is that it is the reflection of pressure from a closer bifurcation in the arterial tree, likely the Circle of Willis. For those individuals whose BAM readings did not have two reflected waves, it is possible that the earlier wave reflection superimposed into the second reflected wave.

To explain more clearly, wave reflections as seen in traditional arterial pressure waves obtained via the radial pulse are the result of a pressure reflection when the descending aorta splits into the left and right iliac arteries in the abdomen. The arterial pressure waveform (Figure 4a) will display a single systolic peak and a single reflected wave, the latter of these resulting from a pressure wave that has traveled from the bifurcation of the descending aorta. In the cerebrum, the reflected wave reaches the BAM sensors more quickly because the distance from the location of the bifurcation of the descending aorta is approximately 1 meter shorter than that to the radial pulse site (Figure $4 b)$. The second reflected wave that we often saw in our subjects, which arrived between the systolic peak and the wave reflection from the aortic bifurcation, is potentially caused by blood flowing through the Circle of Willis (Figure 4c). Following the arterial tree, blood will ascend from the aortic arch into the carotids and then proceed through the 
Circle of Willis. Much of the flow then continues on to the BAM sensors through the anterior cerebral arteries while the rest is directed to other parts of the brain through the posterior and middle cerebral arteries. Some of the flow will remain in the Circle of Willis and, theoretically, get reflected off of major junctions, such as that of the anterior cerebral arteries and the internal carotids. The reflections from this bifurcation only travel, at most, 3 or $4 \mathrm{~cm}$ further than the systolic pulse and therefore arrive at the BAM sensor very close to the first peak and prior to the reflection from the aortic bifurcation.

With regard to our measures, augmentation index and peak interval rely on appropriate identification of the wave reflection and systolic peaks. For those subjects that had a second reflection present, it is possible that the first reflected wave was variably higher than the systolic peak, causing our program to identify the reflection as the systolic peak in some cases and not in others. Either way, better identification of these peaks is necessary to ensure reliability with these measures. Applying these findings to clinical cases of sport related concussion may be possible using presence and absence of the primary and secondary reflections compared to a baseline measure. For instance, if an individual does not show signs of two reflections at baselines, suggesting superposition of the primary and secondary reflections, but two reflections are present at post-injury testing, it is possible that arterial stiffening has caused the primary reflection to reflect early. Alternatively, if two reflections are present at baseline but not at post-injury assessment, it is possible that the same phenomenon has caused the primary reflection to superimpose onto the systolic peak. In either case, it is of utmost importance that baseline data be obtained for these measures to provide potentially useful information. 


\section{Conclusions}

Reliability of the BAM measures are poor to moderate, with systolic interval and steepest rise gradient displaying more favorable reliability coefficients. There is a great degree of variability in augmentation index and peak interval measures likely due to the presence of an anomalous second wave reflection. Once better understanding is held concerning the role of each wave reflection within the arterial pressure waveform obtained by the BAM, reliability should be reassessed. The use of this device in assessing concussion is not supported by these results. 
Table 1. Test-retest Intra-Class Correlations for Left and Right BAM Variables.

\begin{tabular}{|c|c|c|c|c|c|c|}
\hline & \multicolumn{2}{|l|}{ Mean \pm SD } & \multirow[b]{2}{*}{ ICC $(95 \% \mathrm{CI})$} & \multirow[b]{2}{*}{ Spearman's $\rho$} & \multicolumn{2}{|l|}{ SEM } \\
\hline & Day 1 & Day 2 & & & Day 1 & Day 2 \\
\hline Left SI & $0.14 \pm 0.05$ & $0.16 \pm 0.07$ & $0.02(-1.66,0.61)$ & 0.041 & 0.05 & 0.07 \\
\hline Right SI & $0.16 \pm 0.07$ & $0.17 \pm 0.06$ & $0.73(0.30,0.90)$ & $0.548^{* *}$ & 0.04 & 0.03 \\
\hline Left SRG & $3.14 \pm 1.6$ & $3.01 \pm 1.22$ & $0.74(0.33,0.90)$ & 0.279 & 0.82 & 0.62 \\
\hline Right SRG & $3.32 \pm 1.52$ & $3.28 \pm 1.75$ & $0.77(0.41,0.91)$ & $0.558^{* *}$ & 0.73 & 0.59 \\
\hline Left PI & $0.14 \pm 0.05$ & $0.15 \pm 0.04^{*}$ & $0.74(0.32,0.90)$ & 0.243 & 0.03 & 0.02 \\
\hline Right PI & $0.14 \pm 0.06$ & $0.15 \pm 0.05$ & $0.65(0.09,0.87)$ & 0.406 & 0.04 & 0.03 \\
\hline Left AIx & $10.0 \pm 15.8$ & $17.2 \pm 14.8$ & $0.48(-0.36,0.80)$ & $0.494^{* *}$ & 11.4 & 10.7 \\
\hline Right AIx & $12.8 \pm 9.65$ & $12.0 \pm 9.4$ & $0.69(0.19,0.88)$ & $0.640^{* *}$ & 5.37 & 5.23 \\
\hline
\end{tabular}

SI: Systolic Interval; SRG: Steepest Rise Gradient; PI: Peak Interval; aix: Augmentation Index; ICC: Intra-class Correlation Coefficient; SEM: Standard Error of Measurement; *indicates significant difference from Day 1 measure, $\mathrm{p} \leq 0.05$. **indicates significant correlation coefficient, $\mathrm{p} \leq 0.05$. 


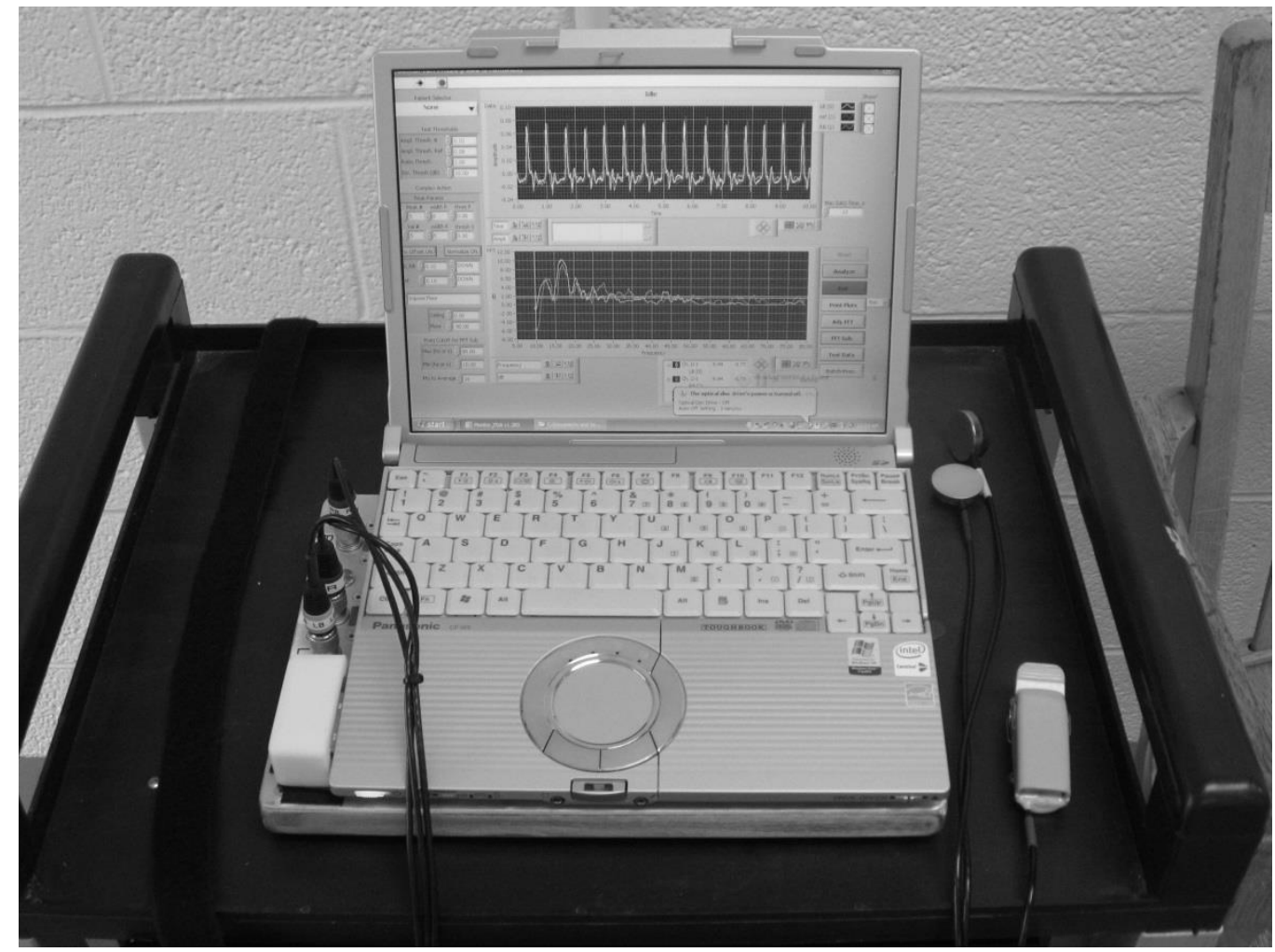

Figure 1. Brain Acoustic Monitor laptop and analog-to-digital convertor with sensors attached. 


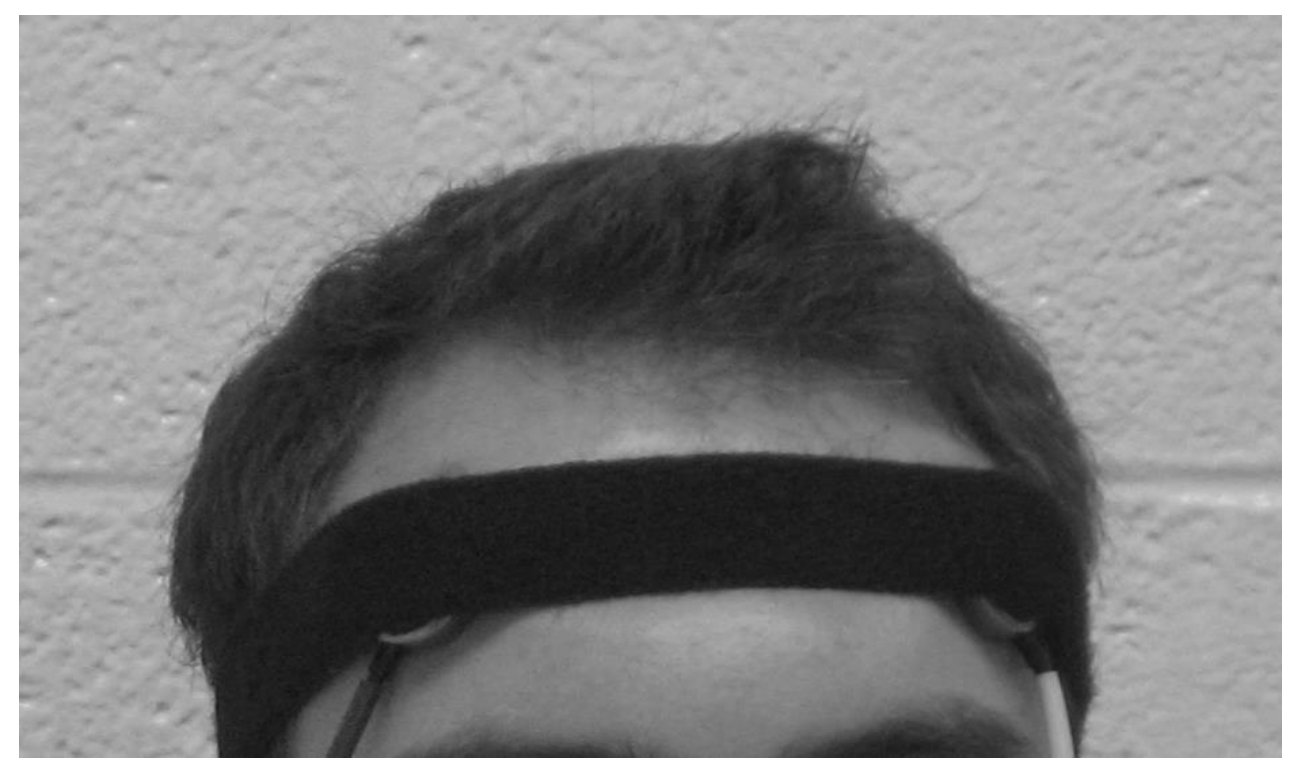

Figure 2. BAM sensors applied to the forehead.

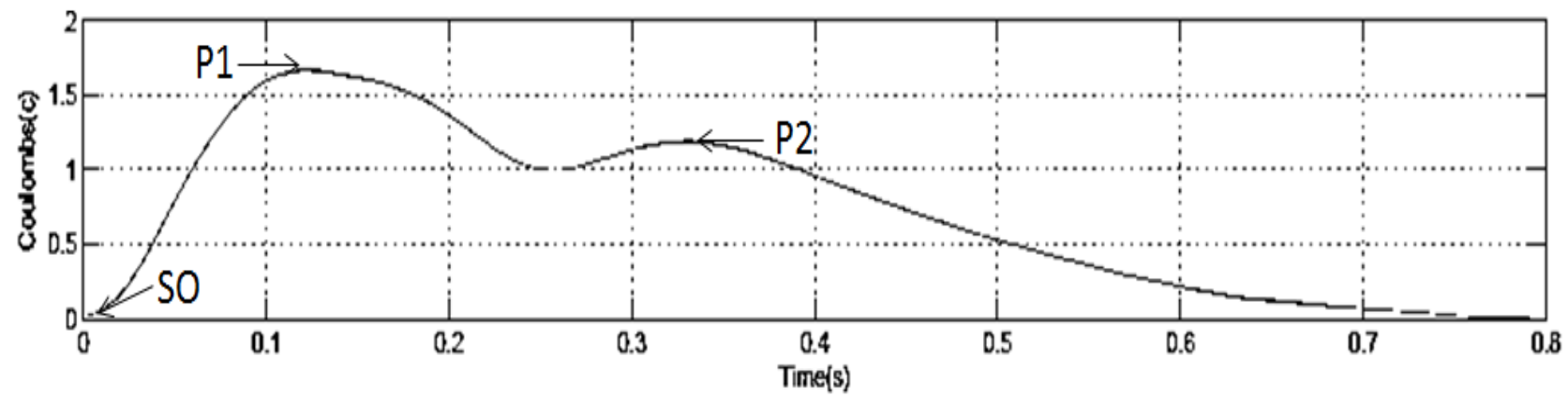

Figure 3. Example arterial pulse wave. $\mathrm{P} 1=$ Peak systolic pressure; $\mathrm{P} 2=$ peak of reflected wave; $\mathrm{SO}=$ systolic onset. 

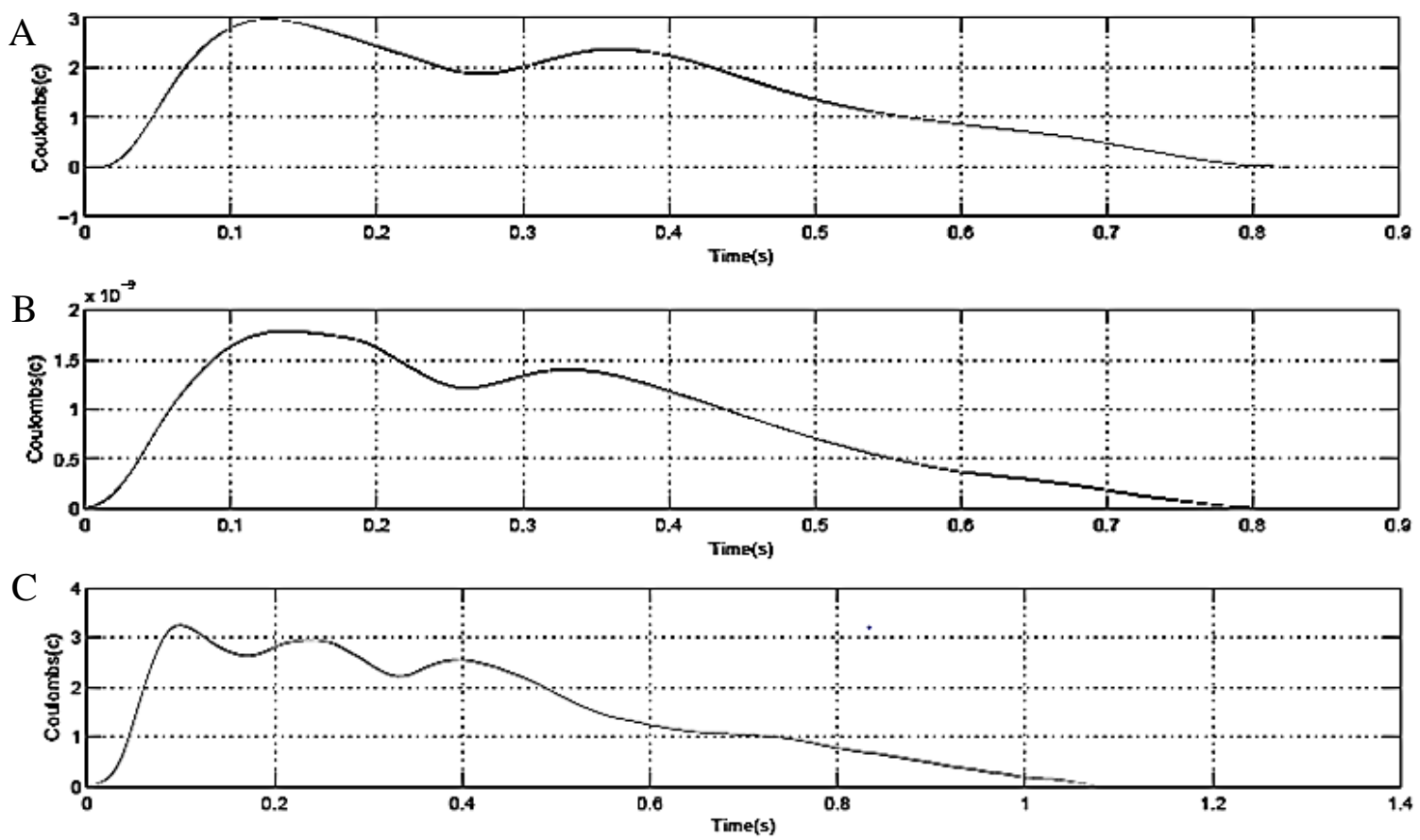

Figure 4. Comparison of radial arterial pressure wave and BAM forehead signals. (A) Normal arterial pressure waveform from radial artery. (B) Expected pressure wave obtained from forehead signals. (C) Pressure wave containing double wave reflections. 


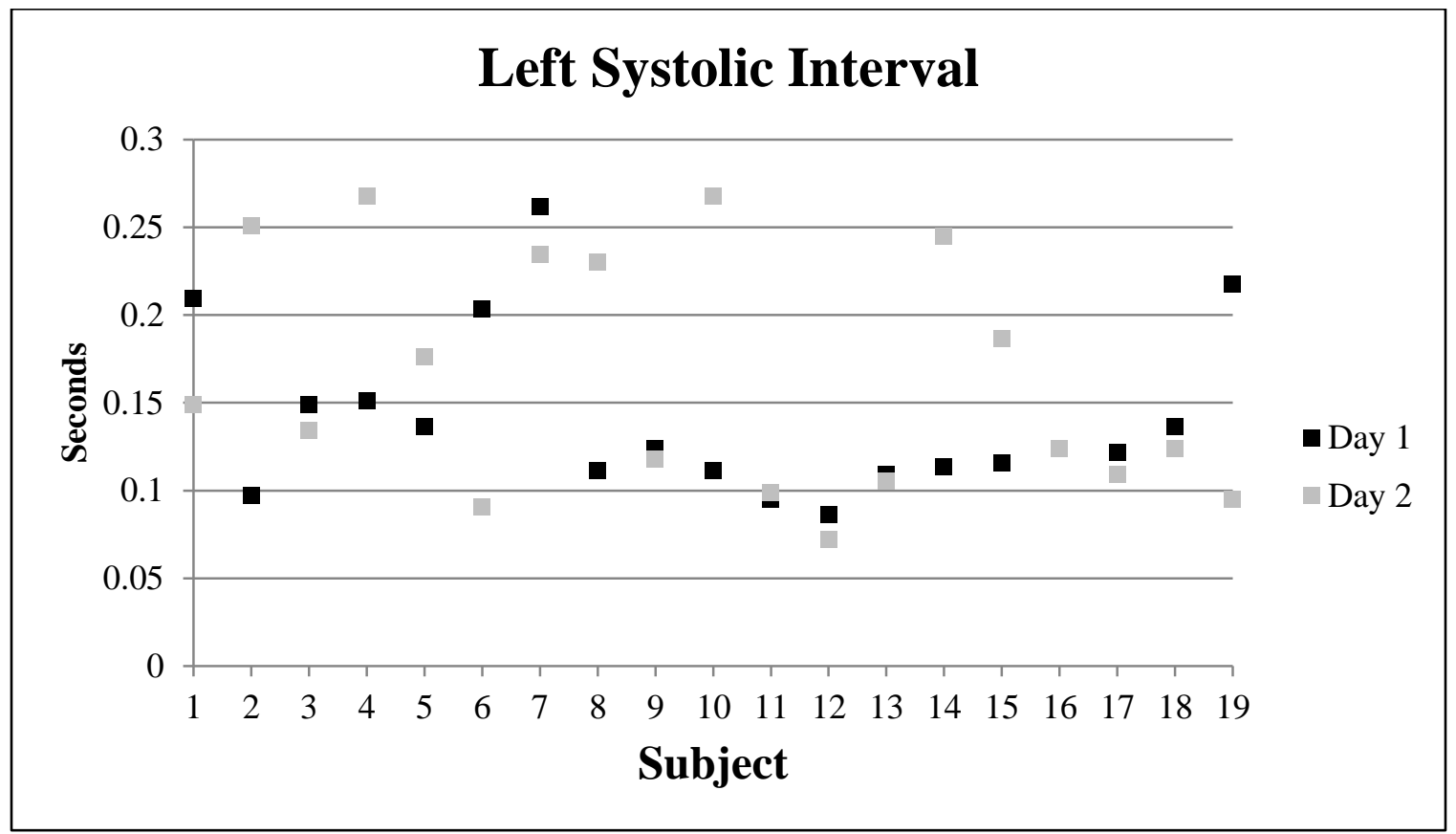

Figure 5. Individual subject values for left systolic interval values on day 1 (black markers) and day 2 (gray markers). 


\section{Section II}

\section{Manuscript II}

Natural History of Cerebral Blood Flow Changes Following Sport-Related Concussion in High School and Collegiate Athletes Using the Brain Acoustic Monitor. 


\begin{abstract}
Context: A need exists for non-invasive tools of objective physiological diagnosis of concussion that can be used in the field. The Brain Acoustic Monitor, or BAM, may offer a solution through detection of changes in the acoustic signals emitted by the cerebral vasculature. Objective: To determine if changes in cerebral blood flow can be detected using the BAM and if these changes persist at resolution of symptoms and return-toactivity. Design: Case-control, repeated measures. Setting: Sports Medicine Clinic. Patients or Other Participants: 17 concussed collegiate and high school athletes (10 males, 7 females; age: $18.8 \pm 2.1$ years) were recruited and matched to 17 healthy controls by age, gender, and sport contact category (age: $18.9 \pm 1.8$ years).

Intervention(s): Concussed subjects were recruited within 24-48 hours of their injury (average $=\mathrm{X}$ ) and SCAT3 and BAM testing were performed at 3 time points: 24-48 hours post-injury, resolution of symptoms, and return-to-play as determined by the team athletic trainer or physician. Healthy controls were assessed with the BAM and SCAT3 prior to the athletic season. Main Outcome Measures: The BAM records acoustic signals emitted by the cerebral vasculature and displays an arterial pressure wave. Outcomes extracted included systolic interval(SI), steepest rise gradient(SRG), peak interval(PKInt), and augmentation index(AIx). Subcomponents of the SCAT3 that were used for comparison included the Standardized Assessment of Concussion(SAC), Modified Balance Error Scoring System (BESS), total symptoms reported(TS), and symptom severity(SS). Within-subject comparisons were made for the concussed group between each time point. These values were also compared to healthy group values. Because data were non-normally distributed, Friedman's Rank test and follow-up Wilcoxon Signed-Rank tests were used for within-group comparisons and Mann-Whitney $\mathrm{U}$ tests were used for between-group comparisons. Significance was set a priori to $\mathrm{P} \leq$ 0.05. Results: No significant differences were found between any time points for all BAM outcomes within the concussed group (Left SI: $p=0.32, \chi^{2}=2.303$; Left SRG: $\mathrm{p}=0.39, \chi^{2}=2.235$; Left PKInt: $\mathrm{p}=0.465, \chi^{2}=1.529$; Left AIx: $\mathrm{p}=0.662, \chi^{2}=0.824$; Right SI: $\mathrm{p}=0.293, \chi^{2}=2.455$; Right SRG: $\mathrm{p}=0.390, \chi^{2}=1.882$; Right PKInt: $\mathrm{p}=0.685, \chi^{2}=0.758$; Right AIx: $p=0.465, \chi^{2}=1.529$ ). Right SI was significantly higher in the concussed group at initial assessment $(\mathrm{p}=0.042, \mathrm{Z}=-2.035)$ and symptom resolution $(\mathrm{p}=0.016, \mathrm{Z}=-2.415)$ than the healthy group (mean rank difference: 6.94 at initial assessment; 8.24 at symptom resolution). These findings were repeated for the BESS at initial assessment with the concussed group displaying significantly greater errors $(\mathrm{p}=0.011, \mathrm{Z}=-2.553$; Concussed: 25.5 \pm 3.0 errors; Healthy: 27.9 \pm 2.2 errors). No differences were found between groups for SAC scores at initial assessment or symptom resolution, but the concussed group performed significantly better than the healthy controls at return-to-activity $(\mathrm{p}=0.035$, $\mathrm{Z}=-2.121$; Concussed: 28.2 \pm 3.0 ; Healthy: 26.5 \pm 2.2 ). Conclusions: This study suggests limited utility of the BAM in concussion management. Because minor differences were found compared to healthy individuals, it is likely the device is not sensitive enough to detect changes following concussion.
\end{abstract}

Key Words: recovery, mild traumatic brain injury, diagnosis, assessment 


\section{INTRODUCTION}

Sports concussions have become a prominent focus in the media as recognition of the injury has increased through athlete and parent educational programming in schools and athletic leagues. This increased recognition has been accompanied by a similar increase in the realm of sports medicine research on various methods of concussion diagnosis and management. With as many as 3.8 million sports-related head injuries occurring each year, there is an obvious need for such a response in the sports medicine community. ${ }^{1}$

Concussion leads to a well-documented cascade of neurometabolic events that result in the presentation of clinical signs and symptoms, such as headache, dizziness, amnesia, and difficulty concentrating. ${ }^{6,8,9,19}$ Current diagnostic methods use a multifaceted approach including symptom scales, pre- and post-injury neuropsychological testing, and balance assessment. ${ }^{3-5}$ The only truly objective measure of physiological function used currently occurs through neuroimaging, and, most often, findings are negative. One area of physiological change following concussion that has not received much attention in the way of diagnosis and management is that of cerebral blood flow. Alterations in the ability of the cerebral arteries to respond appropriately to changes in concentrations of carbon dioxide have been documented following concussion beyond resolution of baseline neurocognitive functioning..$^{20,21}$ This phenomenon, known as cerebrovascular reactivity, is important for maintenance of an adequate supply of oxygen to the recovering brain tissue following injury and provides adequate evidence to suggest a need for gradual return to play. Additionally, research in pediatric populations has 
shown reductions in cerebral blood flow with only $27 \%$ of patients reaching control levels 2 weeks after injury and $60 \%$ of patients reaching control levels 30 days after injury. ${ }^{22}$ Potential mechanisms for these alterations in cerebral blood flow have been suggested to include interruptions in cerebral autoregulation, arterial vasospasm (increased arterial stiffness), and/or extensive regional perfusion perturbations. ${ }^{20,23-29}$ Methods to address these potential pathomechanisms immediately after injury and serially during recovery are needed.

The Brain Acoustic Monitor, or BAM, is an investigational device that may provide a method of monitoring arterial stiffness after concussion. By quantifying arterial perfusion pressure through the acoustic signals emitted by the arteries in the cerebrum, it creates arterial pressure waveforms, similar to that given by an arterial line. Characteristic changes in arterial pressure waves have been identified in various pathologies, namely cardiovascular disease and hypertension. ${ }^{16,30,31}$ Analysis of the pulse contour has become of interest in these populations and measures of diastolic and systolic function have been developed for identification of risk, though none have been examined following concussive injury. ${ }^{16,30,32,33}$ With the thought that changes seen in cerebral blood flow are potentially caused by vasospasm and increased stiffness of the arteries in the brain, use of similar outcomes in concussion diagnosis and management may prove useful.

The BAM was developed at the R Adams Cowley Shock Trauma Center of the University of Maryland and has mainly been studied in emergency room settings with a severely head injured patient population. ${ }^{12,14,15}$ One documented study has been performed in mild traumatic brain injured patients, again in an emergency room setting, 
and the researchers merely correlated BAM findings with computed tomography and expert panel adjudication. ${ }^{13}$ This study used a set of outcomes that dichotomized subject diagnosis as "concussed" or "healthy" by setting thresholds for measures of the raw signal's erraticism and frequency distribution. This study did not use a pressure wave analysis approach and did not quantify changes in blood flow beyond the immediate postinjury assessment. Therefore, the purpose of this study was to use the updated BAM signal processing methods to characterize changes in arterial stiffness following concussion at important clinical time points, such as symptom resolution and return-toactivity, and secondarily, to determine if these values differ from that obtained from healthy controls. We hypothesized that concussed individuals would exhibit increased arterial stiffness that resolved over the recovery period.

\section{METHODS}

A repeated measures case-control design was used to determine how cerebral blood flow measures change following concussion. There was one within-subjects factor at three levels (time: baseline, immediate post-injury, and 24 hours post-injury) and one between-subjects factor at two levels (group: concussed vs. healthy).

\section{Subjects}

Thirty-four high school and collegiate athletes were recruited from two universities and a single private high school. Upon diagnosis by a certified athletic trainer or team physician according to the 2008 International Committee on Concussion in Sport Consensus guidelines, study investigators were contacted and an initial assessment scheduled. Concussed individuals were matched to healthy controls by sport contact 
category (collision, contact, limited-contact, or noncontact), age, and gender. To be included, all subjects must have been participating in varsity or junior varsity athletics at their respective institutions and undertaken baseline neurocognitive testing prior to the athletic season. Subjects were excluded if they reported history of head injury within the past 6 months, if they had known neurological or learning disorders that would affect balance or cognition, or if they had a history of seizures. This study was approved by the university's human subjects research Institutional Review Board (IRB HSR\#16857) and appropriate consent and assent forms were signed by all subjects and parents, as applicable, prior to beginning study procedures.

\section{Instruments}

The BAM (Active Signal Technologies, Inc.; Baltimore, MD) consists of two small circular sensors that are affixed to the forehead and a reference sensor embedded in a finger clip that attaches to the thumb or index finger (Figure 6). Through these sensors, the BAM is able to measure and display the amplitude and frequency distributions of the intracranial pulse signals compared to the reference arterial signal. Readings with the BAM last approximately 10 seconds and are appended in individual files for later processing using a customized transfer function.

Athletes were also administered the Sideline Concussion Assessment Tool version 3 (SCAT3), which was developed by the Concussion In Sport Group in 2004 and updated to its third edition at the 2012 International Consensus Conference on Concussion in Sport, held in Zurich. ${ }^{6}$ This test is designed to be used as a sideline assessment tool and consists of a 22-item graded symptom assessment, a series of 
objective questions regarding physical signs associated with concussion, the GlasgowComa Scale (GCS), Maddocks et al. questions to assess orientation, the Standard Assessment of Concussion (SAC) to assess orientation, immediate memory, concentration, and delayed recall, the modified BESS to assess postural control, and a coordination examination. Administration of this test takes approximately 5 minutes.

\section{Testing procedures}

All subjects presented to their institution's athletic training room for all SCAT3 and BAM testing. Baseline SCAT3 assessments were performed in an isolated location by a certified athletic trainer experienced in its administration and scoring. For concussed athletes, BAM and SCAT3 testing occurred at three time points. The initial assessment occurred within 48 hours of the injury (mean time: $1.2 \pm 0.4$ days). Second and third assessments were performed at symptom resolution and return-to-play. Return-to-play was determined by the athlete's athletic trainer or team physician and occurred at the conclusion of the graded return-to-play protocol. For healthy controls, SCAT3 and BAM testing occurred during their preseason training.

For BAM testing, the BAM sensors were first applied to the forehead, along the lateral border of the eyebrows midway between the eyebrows and hairline. Subjects were asked to close their eyes and to remain as still as possible during testing. During the first 3-5 readings, the right and left sensors were adjusted for optimal placement based on wave amplitude (greater than $0.1 \mathrm{~V}$ ) and absence of aberrant noise or movement artifact. Once proper placement was found, 10 trials were taken for later processing. 
For SCAT3 testing, Maddock's et al. questions, physical signs, and the Glascow Coma subsections were not addressed as these are intended for on-field assessment immediately following concussive injury. The symptom inventory section was filled out by each athlete individually and a clinician reviewed any excessive ratings with the athlete to ensure that they were not the result of comorbidities. Administration of the SCAT3 and BAM measurements took approximately 15 minutes.

\section{Data Processing}

An individual file was created for each 10 second BAM reading. Three of the ten readings were selected for further analysis using wave amplitude and lack of aberrant noise or movement artifact as selection criteria. Data were imported into MatLAB and processed using a custom transformation algorithm to allow for calculation of pressure pulse waves. An averaged pulse wave was created for each of the three selected trials and our dependent variables were calculated by manually identifying the systolic peak (P1), the inflection point or peak of the reflected wave (P2), and systolic onset (SO). These peaks are described in an example in Figure 7. Outcomes used for comparison withinand between-group comparisons included systolic interval, steepest rise gradient, peak interval, and augmentation index. Equations for calculation of systolic interval, peak interval, and augmentation index are presented below:

$$
\begin{gathered}
\text { Systolic Interval }(S I)=\text { Time }_{P 1}-\text { Time }_{S O} \\
\text { Peak Interval }=\text { Time }_{P 2}-\text { Time }_{P 1} \\
\text { Augmentation Index }=\frac{\text { Amplitude }_{P 1}-\text { Amplitude }_{P 2}}{\text { Amplitude }_{P 1}} \times 100
\end{gathered}
$$


Systolic interval represents the time between systolic onset and the systolic peak. As such, a decrease in systolic interval would indicate increased arterial stiffness. Similarly, peak interval is represented by the time between the systolic peak (P1) and the reflected wave (P2). Theoretically, a decrease in time between these two peaks indicates increased pulse wave velocity, an indirect marker of increased arterial stiffness. The magnitude of the reflected wave is represented by augmentation index and increases in this measure (indicated by a decreased augmentation index) represent worsening arterial stiffness and greater pressure within the arteries. Finally, steepest rise gradient represents the greatest slope between systolic onset (SO) and systolic peak (P1).

Scores for the Standardized Assessment of Concussion (SAC), modified Balance Error Scoring System (BESS), Total Symptoms, and Symptom Severity portions of the SCAT3 were calculated and used for within- and between-group comparisons. For the SAC and BESS, subjects could score a maximum of 30 points with a higher score reflecting better performance. For Total Symptoms, the total number of symptoms endorsed was used as a score with a maximum of 22 points possible and lower values indicating fewer symptoms. Subjects rated each symptom on a 0 to 6 scale and these ratings were summed for all reported symptoms as a measure of symptom severity. The total possible points for symptom severity was 132 with a lower value indicating lower symptom severity.

\section{Statistical analysis}

Following testing of normality for BAM and SCAT3 outcomes, a non-normal distribution was identified and we chose to use non-parametric statistical tests to compare 
within- and between-group differences. Means, medians, and interquartile ranges were calculated for the concussed group at 24-48 hours post-injury, symptom resolution, and return-to-activity, and for the healthy group at baseline. Comparison of within-subject differences in BAM and SCAT3 outcomes was performed using Friedman's Rank Test with post-hoc Wilcoxon Signed-Rank tests as indicated. Between-group differences were examined using Mann-Whitney U Tests. Significance was set a priori to $\mathrm{p} \leq 0.05$ and all statistical analyses were performed using IBM SPSS Statistical Software v.20 (IBM SPSS, Inc., Chicago, IL).

\section{RESULTS}

Subject demographics, breakdown by sport contact category, and concussion history data are presented in Table 1 . No significant differences were found between concussed and healthy individuals for age, height, or weight (age: $\mathrm{t}=-0.18, \mathrm{p}=0.86$; height: $\mathrm{t}=0.84, \mathrm{p}=0.41$; weight: $\mathrm{t}=0.16, \mathrm{p}=0.88$ ).

\section{SCAT3 Outcomes}

The concussed group performed significantly worse on the SAC at initial assessment than at symptom resolution $(\mathrm{Z}=-2.136, \mathrm{p}=0.033)$ and return-to-play $(\mathrm{Z}=-$ 2.533, $\mathrm{p}=0.011$ ). Similar findings were seen for total symptoms and symptom severity, with significantly greater symptoms and greater severity reported at initial assessment than symptom resolution (total symptoms: $\mathrm{Z}=-3.185, \mathrm{p}=0.001$; symptom severity: $\mathrm{Z}=-$ 3.18, $\mathrm{p}=0.001$ ) and return-to-play (total symptoms: $\mathrm{Z}=-3.184, \mathrm{p}=0.001$; symptom severity: $Z=-3.182, p=0.001)$. Differences were also seen with these outcomes between symptom resolution and return-to-activity (total symptoms: $\mathrm{Z}=02.214, \mathrm{p}=0.027$; symptom 
severity: $Z=-2.226, p=0.026)$. No differences were found between any time point for the $\operatorname{BESS}\left(\chi^{2}=5.81, \mathrm{p}=0.055\right)$.

Between group comparisons revealed a significantly greater number and more severe symptoms and a greater number of BESS errors at initial assessment in the concussed group compared to the healthy group at baseline (total symptoms: $Z=-3.423$, $\mathrm{p}=0.001$; symptom severity: $\mathrm{Z}=-3.646, \mathrm{p}<0.001 ; \mathrm{BESS}: \mathrm{Z}=-2.553, \mathrm{p}=0.011)$.

Interestingly, the concussed group displayed better SAC performance at return-to-play compared to healthy controls $(Z=-2.121, p=0.035)$, but no differences were found at other time points (initial assessment: $\mathrm{Z}=-1.283, \mathrm{p}=0.211$; symptom resolution: $\mathrm{Z}=-0.517$, $\mathrm{p}=0.628$ ). Means, medians, and interquartile ranges are presented in Table 2.

\section{BAM Outcomes}

No differences were found within the concussed group between initial assessment, symptom resolution, and return-to-play for any BAM outcomes. Means, medians, and interquartile ranges are presented for left and right systolic interval, steepest rise gradient, peak interval, and augmentation index for the concussed group in Table 3. When comparing concussed group values to healthy controls, a significant difference was found in right systolic interval at initial assessment $(\mathrm{Z}=-2.035, \mathrm{p}=0.042)$ and symptom resolution $(\mathrm{Z}=-2.415, \mathrm{p}=0.016)$. Specifically, the concussed group had a longer systolic interval at initial assessment and symptom resolution than the healthy controls. No other differences were found between groups. 


\section{DISCUSSION}

The primary findings of this study were that there were no significant changes in cerebral perfusion pressure in concussed individuals at 24 to 48 hours post-injury, symptom resolution, or return-to-activity. Concussed individuals did, however, differ from healthy controls when comparing right systolic interval values at initial assessment and symptom resolution. Specifically, concussed individuals displayed significantly longer systolic intervals than healthy controls at both time points, potentially suggesting persisting changes in arterial stiffening at resolution of symptoms. It should be noted, however, that a decrease in systolic interval was expected in response to concussion and the opposite phenomenon was seen. That is, systolic interval was greater in concussed individuals compared to healthy controls, indicating a greater time for the systolic peak to occur and theoretically symbolizing a lower level of arterial stiffness and decreased pulse wave velocity. This does not support our hypothesis that concussion would result in increased arterial stiffness that recover by the return to play assessment.

No differences were found within the concussed group at any of the three time points presented here. One explanation is that the blood flow alterations and any abnormal response of the blood vessels within the cerebrum may resolve more quickly than expected. That is, prior research has shown alteration in cerebral blood flow, via volume changes, well beyond the time line presented here. Maugans et al. performed a study in pediatric patients using magnetic resonance phase contrast angiography, which

uses contrasts between flowing blood and stationary tissues to assess flow volume rate. ${ }^{22}$ While these outcomes are both likely altered following concussion, flow volume rate is 
not assessed by the BAM and it is possible the changes seen following concussion are too small to be detected acoustically. Additionally, the BAM strives to assess minute physiologic phenomena regarding stiffness of the arterioles in the cerebrum that should result in increased pulse wave velocity and reduced flow. It is possible that the changes in arterial diameter needed to show changes using BAM outcomes are too small to be detected acoustically and the resultant changes in blood flow rate and volume are more easily detected following injury.

Previous research has suggested presence of reduced blood vessel response to alterations in systemic blood pressure following mild head injury. ${ }^{26,34-37}$ A study by Junger et al. examined the response of the cerebral vasculature in mildly head injured patients to mechanically induced reductions in systemic blood flow. ${ }^{34}$ Large blood pressure cuffs were placed around both thighs and inflated to 20 to $40 \mathrm{mmHg}$ above the systolic pressure for 3 minutes. Releasing the cuffs caused a sharp but moderate transient drop in systemic blood pressure and an accompanied a drop in cerebral blood flow. In normal individuals this drop should be remedied by rapid reduction in cerebrovascular resistance to restore blood flow to the resting levels. These authors found a reduced response in 8 of their mildly head-injured patients and this difference was correlated with reduced cerebral perfusion pressure. It is possible, in relation to our findings, that there were no significant differences between groups or time points because we did not induce any alteration in systemic blood pressure to truly warrant a response by the cerebrovasculature to restore normal blood flow in the brain. 
Continuing to examine normal cerebrovascular reactions to changes in systemic and local physiology, it is also possible that alterations in the partial pressure of $\mathrm{CO}_{2}$ may have been needed to detect a deficit in the ability of the arterioles in the cerebrum to respond. A study by Len et al. used repeated breath holding and hyperventilation to elicit changes in PACO2 levels in a group of concussed athletes an average of 4.5 days postinjury and compared the response to that obtained in healthy controls. ${ }^{20,38}$ They found similar resting values between groups but the velocity of blood flow in the middle cerebral artery (vMCA), measured by transcranial Doppler ultrasonography, was significantly reduced in concussed individuals compared to healthy controls after the second of five periods of hyperventilation. Also, they found that the concussed group was unable to return to baseline vMCA after the third, fourth, and fifth periods of breath holding. The authors attributed these differences to alterations in cerebrovascular reactivity.

Similar findings have been reported in concussed athletes who have become neurocognitively recovered. A study of junior ice hockey players who sustained a concussion and became asymptomatic in approximately 2 days found no significant differences in resting heart rate compared to healthy individuals, but elicited abnormal heart rate variability in response to submaximal exercise. ${ }^{39}$ The shift from dominating parasympathetic control of heart rate at rest to sympathetic control during exercise suggests that since differences are seen following exertion, a disruption or uncoupling of the sympathetic response of the autonomic system and cardiovascular response is present. This may have implications for concussed individuals entering the return to play protocol 
after becoming asymptomatic when, in fact, alterations or uncoupling of these systems has been shown to last 10 days post-injury.

The premise behind the changes we expected to see in this study are based on former studies of more severe brain-injured patients, which has shown cerebral vasospasm to be a significant predictor of clinical outcome, independent of severity upon admission and age. ${ }^{40}$ We chose not to induce any systemic blood pressure or hyper/hypocapnic stresses on our concussed subjects because we wanted to address the question of whether or not cerebral vasospasm might be occurring following concussion at rest and, if so, did these changes outlast other measures of recovery such as symptomology and neurocognitive function. While it is possible that alterations may have been seen were we to compare to a baseline measure, the lack of consistent differences in reference to healthy subjects suggests that perhaps no arterial vasospasm or stiffening occurs following concussion. This is potentially explained by the sample of concussed individuals assessed in this study. The return to activity ranged from 5 days to three weeks, largely showing an "uncomplicated" sample of concussed athletes. The BAM may have more utility in identifying individuals who are at increased risk of prolonged recovery due to comorbidities such as migraine, depression, and prior history of concussion.

Prior studies using the BAM have relied on more basic metrics of the raw acoustic signal and have not focused on sport-related concussion. ${ }^{12-15}$ As such, the data processing methods used to calculate the outcomes used in this study are novel. Criteria for acceptance of a signal as satisfactory were based on metrics of the raw signal and the 
arterial pressure waveforms obtained post-processing rely on accurate acceptance of the raw signal during real-time data collection. Consequently, it may be that the investigator training regarding recognition of a clean signal was not sufficient. As stated in the "procedures" section, study investigators performed a "trial and error" session using the first 3 to 5 readings such that an adequate signal amplitude was obtained and minimal noise was experienced. It may be that there are other forms of signal examination we are not yet aware of that need to be judged for a signal to be ideal for application of the transfer function. Post-processing waveforms were inspected for uniformity and the three cleanest waveforms were selected for analysis, however, some waveforms had additional reflected waves or minimal difference between reflected wave peak and systolic peak (examples depicted in Figure 8). These differences were seen in both concussed and healthy groups.

Future research might include alternative sensor placement sites with hope of finding a cleaner acoustic signal. Studies using transcranial Doppler ultrasonography have focused on basilar arteries by measuring over the temporal region of the skull. ${ }^{41}$ Our sensor placement varied based on skull size and shape as well as signal quality. Interestingly, some individuals displayed cleaner acoustic signals more anteriorly than laterally while others were recorded in the temporal window just above the zygomatic arch. For the device to become useful in a clinical setting, guidelines will be needed regarding variation in skull size and shape such that sensor application is quick and easy and minimal time for trial and error is needed. Comparison of signal quality using 
different BAM sensor placements and transcranial Doppler ultrasound for validation may be necessary.

\section{Conclusions}

The results of this study suggest no presence of arterial stiffness in concussed individuals following concussion. However, it is likely that introduction of systemic blood pressure alterations or variations in partial arterial pressure of carbon dioxide may highlight dysfunction in arterial reactivity or cerebral autoregulation despite lack of differences at rest. The use of the BAM as part of a multifactorial concussion management approach is not yet warranted. 
Table 2. Subject Demographics, Sport Type, and Concussion History for Concussed and Healthy Groups. Time to First Assessment, Symptom Resolution, and Return-to-Play for Concussed Group.

\begin{tabular}{lll}
\hline & \multicolumn{2}{c}{ Group } \\
\cline { 2 - 3 } Variable & Concussed & Healthy \\
\hline Age (years) & $18.8 \pm 2.1$ & $18.8 \pm 1.8$ \\
Height $(\mathrm{cm})$ & $182.1 \pm 25.6$ & $176.5 \pm 11.3$ \\
Weight $(\mathrm{Kg})$ & $77.1 \pm 23.2$ & $75.9 \pm 20.6$ \\
Gender (M:F) & $10: 7$ & $10: 7$ \\
Sport type: & & \\
$\quad$ Collision & $9(52.9 \%)$ & $10(58.8 \%)$ \\
$\quad$ Contact & $6(35.3 \%)$ & $7(41.2 \%)$ \\
$\quad$ Limited Contact & $1(5.9 \%)$ & -- \\
$\quad$ Noncontact & $1(5.9 \%)$ & $11.8 \%$ \\
Concussion History (\%) & $100 \%$ & $15(88.2 \%)$ \\
No. of Past Concussions & -- & $1(5.9 \%)$ \\
$\quad 0$ & $13(76.5 \%)$ & $1(5.9 \%)$ \\
1 & $3(17.6 \%)$ & -- \\
2 & $1(5.9 \%)$ & -- \\
3 & & -- \\
Time to Assessment (days): & $1.2 \pm 0.4$ & -- \\
First Assessment & $5.1 \pm 3.4$ & \\
Symptom Resolution & $10.2 \pm 4.9$ & \\
Return-to-Play & &
\end{tabular}


Table 3. Concussed and Healthy Group scores for Standardized Assessment of Concussion, Modified Balance Error Scoring System, Total Symptoms, and Symptom Severity.

\begin{tabular}{llc}
\hline & & Group \\
\cline { 2 - 3 } Variable & Concussed & Healthy (baseline) \\
\hline SAC & $25.1 \pm 3.1$ & $26.5 \pm 2.2$ \\
$\quad$ Initial Assessment & $27.1 \pm 1.62^{*}$ & \\
$\quad$ Symptom Resolution & $28.2 \pm 2.2^{* *}$ & \\
$\quad$ Return-to-Play & & \\
mBESS & $25.5 \pm 3.0 \ddagger$ & \\
$\quad$ Initial Assessment & $26.4 \pm 2.7$ & \\
$\quad$ Symptom Resolution & $28.4 \pm 1.5$ & \\
$\quad$ Return-to-Play & & \\
Total Symptoms & $8.9 \pm 6.8^{*}$ & \\
$\quad$ Initial Assessment & $1.8 \pm 3.5^{*}$ & \\
$\quad$ Symptom Resolution & $0.29 \pm 0.6^{* \dagger}$ & \\
$\quad$ Return-to-Play & & \\
Symptom Severity & $22.6 \pm 20.9^{*}$ & \\
$\quad$ Initial Assessment & $2.9 \pm 6.1^{*}$ & \\
$\quad$ Symptom Resolution & $0.29 \pm 0.6^{* \dagger}$ & \\
Return-to-Play & indicates significant difference from initial assessment \\
†indicates significant difference from symptom resolution & \\
${ }^{\ddagger}$ indicates significant difference from healthy group &
\end{tabular}


Table 4. Concussed and Healthy Group scores (mean \pm SD) for Left and Right Systolic Interval, Steepest Rise Gradient, Peak Interval, and Augmentation Index.

\begin{tabular}{|c|c|c|}
\hline \multirow[b]{2}{*}{ Variable } & \multicolumn{2}{|c|}{ Group } \\
\hline & Concussed & Healthy (baseline) \\
\hline \multicolumn{3}{|l|}{ Left Systolic Interval } \\
\hline Initial Assessment & $0.13 \pm 0.04$ & $0.11 \pm 0.03$ \\
\hline Symptom Resolution & $0.11 \pm 0.05$ & \\
\hline Return-to-Play & $0.13 \pm 0.05$ & \\
\hline \multicolumn{3}{|l|}{ Right Systolic Interval } \\
\hline Initial Assessment & $0.13 \pm 0.05^{\ddagger}$ & $0.11 \pm 0.04$ \\
\hline Symptom Resolution & $0.12 \pm 0.04^{\ddagger}$ & \\
\hline Return-to-Play & $0.13 \pm 0.04$ & \\
\hline \multicolumn{3}{|c|}{ Left Steepest Rise Gradient } \\
\hline Initial Assessment & $3.6 \pm 1.7$ & $3.5 \pm 2.8$ \\
\hline Symptom Resolution & $3.8 \pm 2.4$ & \\
\hline Return-to-Play & $2.7 \pm 1.3$ & \\
\hline \multicolumn{3}{|c|}{ Right Steepest Rise Gradient } \\
\hline Initial Assessment & $3.13 \pm 2.6$ & $3.0 \pm 1.8$ \\
\hline Symptom Resolution & $3.5 \pm 2.6$ & \\
\hline Return-to-Play & $3.0 \pm 2.0$ & \\
\hline \multicolumn{3}{|l|}{ Left Peak Interval } \\
\hline Initial Assessment & $0.18 \pm 0.07$ & $0.17 \pm 0.6$ \\
\hline Symptom Resolution & $0.18 \pm 0.08$ & \\
\hline Return-to-Play & $0.17 \pm 0.08$ & \\
\hline \multicolumn{3}{|l|}{ Right Peak Interval } \\
\hline Initial Assessment & $0.18 \pm 0.08$ & $0.19 \pm 0.07$ \\
\hline Symptom Resolution & $0.18 \pm 0.09$ & \\
\hline Return-to-Play & $0.20 \pm 0.06$ & \\
\hline \multicolumn{3}{|l|}{ Left Augmentation Index } \\
\hline Initial Assessment & $21.9 \pm 13.8$ & $26.3 \pm 19.8$ \\
\hline Symptom Resolution & $23.5 \pm 18.0$ & \\
\hline Return-to-Play & $22.3 \pm 17.5$ & \\
\hline \multicolumn{3}{|l|}{ Right Augmentation Index } \\
\hline Initial Assessment & $20.0 \pm 18.7$ & $27.7 \pm 16.9$ \\
\hline Symptom Resolution & $27.8 \pm 22.8$ & \\
\hline Return-to-Play & $28.7 \pm 19.7$ & \\
\hline
\end{tabular}




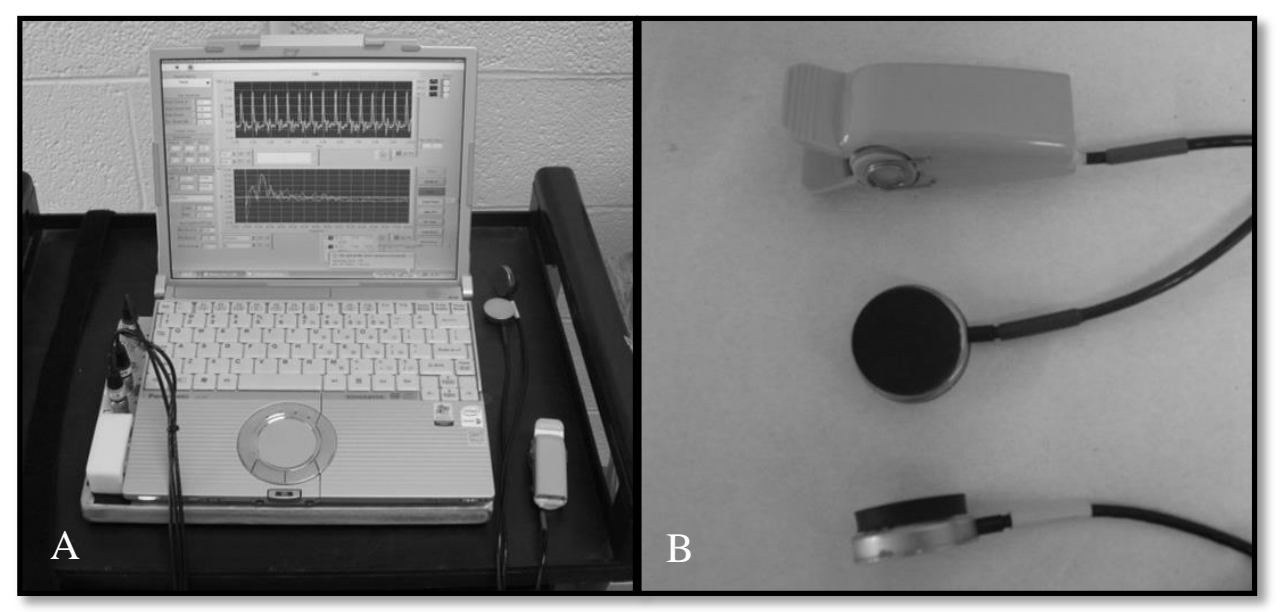

Figure 6. Brain Acoustic Monitor laptop and analog-to-digital convertor (A). Right and left forehead sensors (round sensors) and finger clip used to attach finger sensor (B).

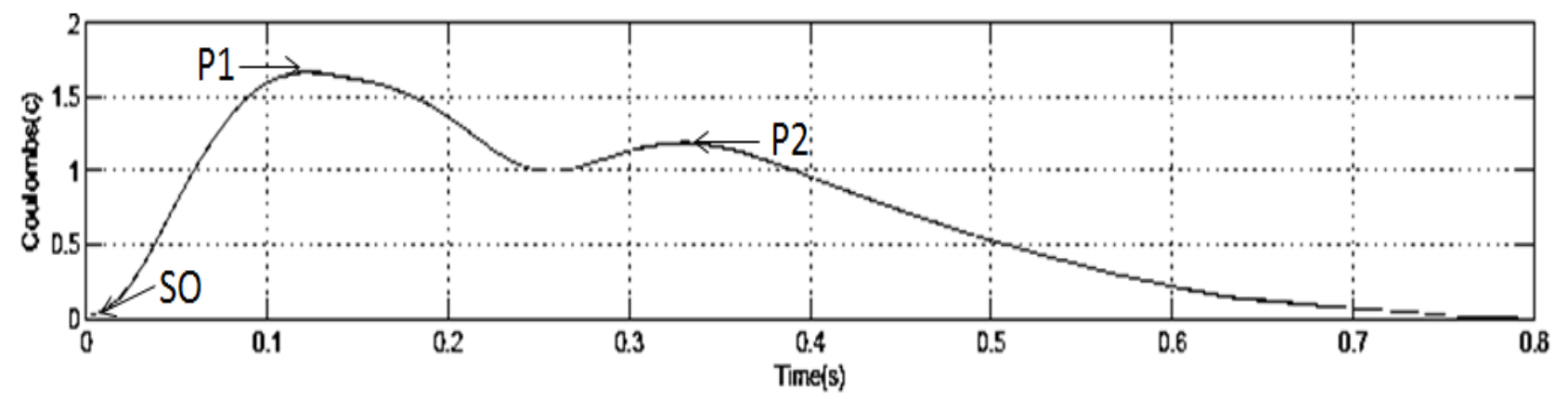

Figure 7. Example arterial pulse wave. $\mathrm{P} 1=$ peak systolic pressure; $\mathrm{P} 2=$ peak of reflected wave; $\mathrm{SO}=$ systolic onset. 

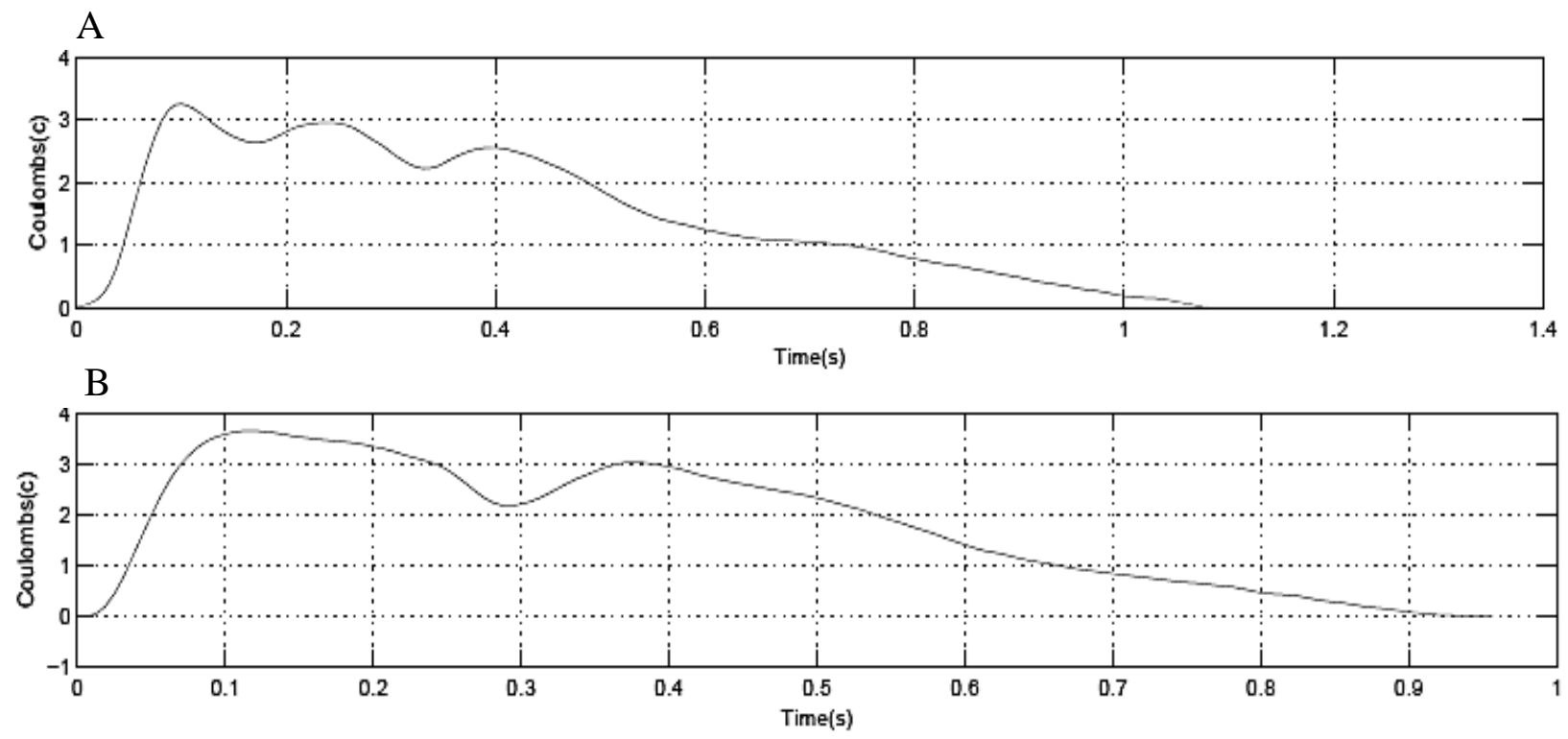

Figure 8. Example waveforms of additional reflected wave (a) or minimal difference between reflected wave and systolic peak (b). 


\section{Section II}

\section{Manuscript III}

Relationship Between Characteristics of Cerebral Blood Flow as Measured by the Brain Acoustic Monitor and Neurocognitive Performance in Healthy and Concussed Athletes. 


\begin{abstract}
Context: It is known that the molecular and metabolic changes associated with concussive injury, such as decreased cerebral blood flow, often exist past resolution of symptoms and recovery of neurocognitive function. It is unknown whether such changes in blood flow are associated with alterations in neurocognitive performance. Objective: To determine whether measures of cerebral perfusion obtained using the Brain Acoustic Monitor are associated with alterations in neurocognitive function. Design: Case-control design. Setting: Athletic Training Room Patients or Other Participants: 16 concussed intercollegiate and high school athletes (10 males, 6 females; age: $20.0 \pm 1.4$ years, height: $175.3 \pm 15.2 \mathrm{~cm}$; weight: $85.4 \pm 31.5 \mathrm{~kg}$ ) and 22 age, gender, and sport-matched healthy controls participated (age: $19.8 \pm 0.9$ years; height: $177.8 \pm 11.9 \mathrm{~cm}$; weight: 82.1 $\pm 22.3 \mathrm{~kg}$ ). Concussed individuals were included after diagnosis by their team athletic trainer or physician. Intervention(s): BAM measurements were taken on all subjects. For concussed individuals, BAM readings coincided with administration of a post-injury CRI (average time of assessment: $4.5 \pm 3.3$ days). Subjects also completed the SCAT3. Main Outcome Measures: Using the mean of three BAM trials, arterial pressure waveforms were created. Dependent variables calculated using these waveforms included systolic interval, steepest rise gradient, peak interval, and augmentation index. Outcomes exported from the CRI included processing speed, complex reaction time, and simple reaction time. Due to the non-normal distribution of our data, Mann-Whitney U tests for independent means was used to compare differences between groups. Spearman's Rho correlations were used to assess relationships between BAM outcomes and neurocognitive test outcomes (z-scores). Those correlations whose associated p-value was less than or equal to 0.10 were entered into a step-wise linear regression model to determine if BAM outcomes were significant predictors of neurocognitive test outcomes. Results: Concussed individuals had a higher right systolic interval compared to healthy controls $(0.12 \pm 0.04$ vs. $0.09 \pm 0.02 ; \mathrm{Z}=-3.0, \mathrm{P}=0.002)$ No other BAM outcomes were different between groups. Right and Left augmentation index (right: $\rho=0.334, P=0.06$; left: $\rho=0.335, P=0.06$ ) and peak interval (right: $\rho=0.349, P=0.05$; left: $\rho=0.381, P=0.032$ ) were entered into a step-wise linear regression model using processing speed as the dependent variable. Left systolic interval was a significant predictor of processing speed $\left(\mathrm{R}^{2}=0.147, \beta=1.788, \mathrm{p}=0.031\right)$. Conclusions: The BAM outcomes used in this study were unable to find consistent differences between healthy and concussed subjects at initiation of graded return to activity. While this may indicate resolution of any blood flow changes that might have occurred following concussion, certain outcomes might be useful in determining if an individual is more susceptible to concussive injury.
\end{abstract}

Key Words: cerebral blood flow, concussion, mild traumatic brain injury 


\section{INTRODUCTION}

Concussion, or mild traumatic brain injury, results in a complex pathophysiological process affecting the brain, consequentially leading to onset of shortlived impairment of neurological function that resolves spontaneously. ${ }^{4,5}$ Involved in this pathophysiological process are a number of metabolic and molecular sequelae including abnormal depolarization of neurons, increased release of excitatory neurotransmitters, ionic imbalances, and hyperglycolysis. ${ }^{6-9,19}$ While these changes are most often transient, the challenge for sports medicine clinicians is to determine when full recovery occurs in an effort to prevent recurrent and/or late-life cognitive deficits.

Current methods that are used for assessing the clinical changes resultant from these metabolic and molecular deficits include symptom reporting, neuropsychological testing, and balance assessment. ${ }^{4,5}$ While these are good clinical indicators of recovery, evidence exists confirming prolonged alterations in brain function at the cellular level well after symptomology, cognitive function, and balance return to baseline levels. ${ }^{10,11,42-}$ ${ }^{46}$ Some forms of neuroimaging have been developed with hope to address these issues, but they do not offer practical applications for sports medicine professionals "in the field" and often expose injured patients to unnecessary radiation.

The Brain Acoustic Monitor, or BAM, is a tool that may offer a solution in the form of a portable measurement of cerebral blood flow. It has been documented that changes in blood flow velocity and alterations in cerebrovascular autoregulation and reactivity are present following concussion. ${ }^{20,22,47-49}$ Reductions in cerebral blood flow up to $50 \%$ have been seen in laboratory models of concussion using rodents ${ }^{50-52}$ and have 
been supported by research in humans with recovery in a large majority of patients occurring greater than 2 weeks post-injury. ${ }^{22}$ These findings suggest a potential relationship between alterations in the metabolic processes in the brain and decreases in cerebral blood flow. Since recovery of these deficits appear to outlast the molecular imbalances associated with concussion, an assessment of cerebral blood flow could offer clinicians a reliable tool for measuring true recovery beyond clinical signs and symptoms.

The BAM was developed at the R Adams Cowley Shock Trauma Center of the University of Maryland and has been studied in various hospital environments including trauma/triage centers, operating rooms, and intensive care units. ${ }^{12-15}$ It has shown excellent sensitivity in diagnosing severe head injuries, justified by computed tomography. ${ }^{14,15}$ However, these findings do not directly transfer to sport-related concussion as the severity and type of brain injury are at opposite ends of the brain injury spectrum. Additionally, recent developments in BAM signal processing methods warrant further examination in this population. Therefore, our purpose was to determine how BAM measures of cerebral perfusion in concussed individuals compare to healthy controls in the sub-acute phase of recovery and whether any differences that do exist correlate with changes on other forms of assessment such as neurocognitive and balance testing.

\section{METHODS}

A case-control design was used to determine if concussed and healthy age and gender-matched collegiate athletes differ in measures of cerebral blood flow as measured 
by the BAM. There was one between-group factor (group: healthy vs. concussed) and no within-group factors.

\section{Subjects}

Subjects were recruited from three universities and a single private high school. 16 concussed individuals (age: $20.0 \pm 1.4$ years; height: $175.3 \pm 15.2 \mathrm{~cm}$; weight: $85.4 \pm$ $31.5 \mathrm{~kg}$ ) were included following diagnosis by their team athletic trainer or team physician, according to guidelines set forth in the 2012 International Consensus Conference on Concussion in Sport. ${ }^{4}$ Healthy, age- and gender-matched controls (height: $177.8 \pm 11.9 \mathrm{~cm}$; weight: $82.1 \pm 22.3 \mathrm{~kg}$ ) were recruited for comparison and excluded if they had experienced a concussion or other head injury in the previous six months. All subjects were excluded if they had not undergone baseline neurocognitive testing prior to athletic participation, if they had a known neurological or learning disorder that might affect balance or cognition, or if they had a history of seizures. This study was approved by the university's human subjects research Institutional Review Board (IRBHSR \#16857) and all subjects, and parents where applicable, gave informed consent/assent prior to beginning study procedures.

\section{Instruments}

The BAM (Active Signal Technologies, Inc.; Baltimore, MD) consists of two small circular sensors that are affixed to the forehead and a reference sensor is embedded in a finger clip that attaches to the thumb or index finger. Through these sensors, the BAM piezoelectrically measures and displays the amplitude and frequency distributions of the intracranial pulse signals and compares them to the reference arterial signal. 
Readings with the BAM lasts approximately 10 seconds and are appended in individual files for later reference. The raw time and frequency domains are retrospectively analyzed to obtain arterial pressure waveforms following transformation using a customized MatLAB routine.

A battery of neurocognitive tests was also used to assess changes in neurocognitive function following concussion. As part of each university's annual preseason testing regime, all athletes were administered the Cognitive Resolution Index (CRI) (Headminder, Inc., New York, NY. This test is a web-based computerized neurocognitive testing battery consisting of multiple subtest modules designed to measure areas of cognitive function including reaction time and processing speed. Administration of the CRI takes approximate 25 minutes. Other tests included in the protocol include the Standardized Concussion Assessment Tool - version 3 (SCAT3), which consists of a 22item symptom scale, a 2-item physical signs score, the Glascow Coma Scale, the Standardized Assessment of Concussion (SAC), modified Balance Error Scoring System (BESS), and a coordination examination.

\section{Testing procedures}

All testing was performed in a quiet, isolated environment located in the university athletic training room. The athletic trainer of the subject's respective team notified study investigators within 24 hours of the injury and assessments of concussed individuals occurred at the time of post-injury neurocognitive testing (average 4.5 days post-concussion). Computerized neuropsychological testing occurred first, followed by BAM testing and administration of the SCAT3. For BAM testing, an elastic strap was 
used to secure each sensor to the right and left forehead, midway between the hairline and eyebrows, along the lateral border of the eyebrows. The reference sensor was applied to the left thumb or index finger (Figure 9). Signal quality was assessed through analysis of 3-5 trials of 10 second BAM readings and sensors were adjusted as needed. Once optimal sensor placement was obtained using wave amplitude (greater than $0.1 \mathrm{~V}$ ) and absence of aberrant noise as acceptance criteria, ten 10 second readings were taken for later processing.

Following BAM assessment, the SCAT3 was administered. Standardized instructions were given to each subject according to the testing guidelines given within the SCAT3 form by a certified athletic trainer experienced in its administration and scoring. Total testing time was approximately one hour.

\section{Data Processing}

Raw acoustic signals obtained by the BAM were recorded and a file for each 10 second reading was created. Three trials were selected for use in data analysis, again using wave amplitude (greater than $0.1 \mathrm{~V}$ ) and absence of aberrant noise or movement artifact as selection criteria. These data were then imported into Matlab for further processing using a custom transfer function. The average of 3 individual waveforms was used for calculation of systolic interval, steepest rise gradient, peak interval, and augmentation index using specific points (SO, P1, and P2) identified in the arterial pressure waveform (Figure 10). Systolic Interval was defined as the time interval between the first peak (P1) and systolic onset (SO). Similarly, peak interval was defined as the time interval between the peak of the reflected wave (P2) and the systolic peak 
(P2). Physiologically, a decrease in both of these measures would indicate increased arterial stiffness. For steepest rise gradient, the highest slope over 10 data points was identified for the segment of the waveform between systolic onset (SO) and the systolic peak (P1). A higher slope would indicate increased arterial pressure applied over a smaller time period, as would be expected following brain injury. Finally, for augmentation index we calculated the difference in magnitude between the systolic peak (P1) and the reflected wave (P2) expressed as a percentage of the systolic peak. Theoretically, as arterial stiffness increases, the pressure attenuated by the arterioles in the cerebrum would be reduced, resulting in a higher reflected wave magnitude. This would be evidenced by a reduction in augmentation index. Equations for the calculation of systolic and peak interval and augmentation index are shown below:

$$
\begin{aligned}
& \text { Systolic Interval }(S I)=\text { Time }_{P 1}-\text { Time }_{S O} \\
& \text { Peak Interval }=\text { Time }_{P 2}-\text { Time }_{P 1} \\
& \text { Augmentation Index }=\frac{\text { Amplitude }_{P 1}-\text { Amplitude }_{P 2}}{\text { Amplitude }_{P 1}} \times 100
\end{aligned}
$$

Using the SCAT3 outcomes, scores for the SAC, BESS, total symptoms, and symptom severity portions of the SCAT3 were calculated. For the SAC and BESS, subjects could score a maximum of 30 points with a higher score reflecting better performance. Errors sustained during the BESS portion were subtracted from 30, with 10 possible errors applicable to each stance of the test. For total symptoms, the total number of symptoms endorsed was used as a score with a maximum of 22 points possible and lower values indicating fewer symptoms. Subjects also rated each symptom on a 0 to 6 
scale and these ratings were summed for all reported symptoms as a measure of symptom severity. The total possible points for symptom severity was 132 with a lower value indicating lower symptom severity.

Extraction of the dependent variables associated with the CRI was performed retrospectively. These variables included complex reaction time, simple reaction time, and processing speed. A lower score for each variable indicates better performance.

\section{Statistical analysis}

Examination of the normality of BAM and neurocognitive test data revealed a non-normal distribution. Therefore, non-parametric tests were used to assess group differences. Between-group comparisons were performed using Mann-Whitney U tests for independent means to indicate differences in BAM outcomes (systolic interval, steepest rise gradient, peak interval, and augmentation index), SCAT3 outcomes (SAC, BESS, total symptoms, and symptom severity), and neurocognitive test outcomes (processing speed, complex reaction time, and simple reaction time) between groups. Significance was set a priori at $\mathrm{p} \leq 0.05$. Spearman's Rho correlations were also calculated between BAM outcomes and pre-existing measures of concussion assessment including SAC, BESS, processing speed, and complex and simple reaction time. All calculations were performed using IBM SPSS Statistical Software v.20 (IBM SPSS, Inc., Chicago, IL).

\section{RESULTS}

Subject demographics, breakdown by sport-contact category, and concussion history are presented in Table 5. No differences were found between the concussed and 
healthy groups for height or weight. The concussed group was assessed an average of 4.5 days post-concussion with a range of 2 to 12 days. Between-group comparisons revealed a significantly greater right systolic interval in the concussed group compared to the healthy controls $(\mathrm{Z}=-3.0, \mathrm{p}=0.003)$. No other differences in $\mathrm{BAM}$ variables were found between groups (Table 6). No significant differences were seen in SAC total score, BESS errors, or total symptoms and symptom severity between groups. These findings were repeated in a comparison of processing speed, complex reaction time, and simple reaction time z-scores (Table 7).

Correlation analyses revealed significant correlations at $p \leq 0.10$ between processing speed and right and left peak interval and augmentation index. No correlations were seen between SAC total score, BESS errors, Total Symptoms, or Symptom Severity and any BAM variables (Table 8). Step-wise linear regression revealed that left peak interval was a significant predictor of processing speed $\left(\mathrm{R}^{2}=0.147, \beta=1.788, \mathrm{p}=0.031\right)$. The three other variables, left augmentation index and right peak interval and augmentation index, were not significant predictors.

\section{DISCUSSION}

The principle purpose of this study was to determine if concussed and healthy individuals differed in measures of arterial stiffness as measured by the BAM at resolution of symptoms. We sought to secondarily assess whether BAM measures predicted poorer neurocognitive function. Our results show a single difference in right systolic interval between concussed and healthy individuals at the clinical time point chosen and no other consistent differences. A significant relationship between left peak 
interval and the processing speed index, as obtained by the CRI, was seen. However, it appears that left peak interval improved with decreased processing speed performance. This would not support our hypothesis that poorer measures of arterial stiffness would predict poor neurocognitive function.

We chose to use measures obtained at symptom resolution because this is the clinical time point at which a graduated return to activity is often initiated and it would be beneficial to know if blood flow differences still exist at this time point. Additionally, according to the university and high school concussion policies used for the subjects included in this study, neurocognitive testing was not performed until symptom resolution. While this is in accordance with recommendations made by the National Athletic Trainers' Association and the most recent consensus statement issued by the International Committee on Concussion in Sport, ${ }^{4,5}$ it did not afford much in the way neurocognitive deficit in our sample and may have been too late after concussive injury to identify differences in arterial function. In other words, it is likely that resolution of arterial vasospasm had occurred prior to our assessment. Additionally, the sample size we used for assessing the predictive value of arterial stiffness measures may not have provided adequate power to accurately reflect the true relationship between BAM measures and neurocognitive test scores.

Regardless of the timing and sample size constraints, we were able to find a positive association between left peak interval and processing speed, but the relationship was not as expected. That is, as peak interval increased, which would indicate improved arterial stiffness by a reduction in pulse wave velocity, processing speed also increased, 
indicating poorer neurocognitive performance. Consequently, these results are in contrast to previous studies examining the relationship between arterial stiffness and neurocognitive function. Pase et al. examined the relationship between arterial stiffness and specific domains of cognitive function in a healthy middle-aged sample and found pulse pressure to be a significant predictor of secondary episodic memory (delayed recall) and both pulse pressure and augmentation index as significant predictors of speed of memory retrieval. ${ }^{53}$ However, an important methodological difference between the current study and that used by these authors was how arterial stiffness measures were obtained. Most studies referring to arterial stiffness to-date use invasive means of pressure recording using a central line or noninvasively via applanation tonometry at the radial or other peripheral arterial site, which involves a strain gauge pressure sensor. Additionally, the BAM measures pressure waveforms more directly at the cerebrum whereas applanation tonometry is used at a peripheral arterial pulse site and is most often mathematically manipulated for derivation of central (aortic) pressure. As such, the authors of the aforementioned study were seeking to describe the influence of systemic arterial stiffness rather than local stiffness, as is described in our study. Therefore, it is likely our results are not directly comparable.

Despite these methodological differences, the theories behind the mechanisms of cognitive decline in the presence of arterial stiffness are similar. Because the brain is continually and passively perfused at high-volume flow, increases in arterial stiffness lead to augmented pressure in the smaller vessels within the brain unlike the main arterial tree where pulsatile energy or pressure is isolated to major arteries. ${ }^{31}$ This increased 
pressure within the small vessels is thought to cause progressive microvascular disease, specifically in areas of the brain that are implicated in memory and learning processes. ${ }^{54-}$ ${ }^{56}$ As such, most studies using systemic measures of arterial stiffness are describing structural and functional changes of vessels that occur with aging. ${ }^{57}$ This was not the expected mechanism of arterial stiffness for the population used in this study. Rather, we sought to describe the relationship between cognitive ability and arterial stiffness that might result in response to concussion. It is known that concussion is largely a diffuse brain injury resulting in shearing and tensile forces within white and gray matter. We suspected microvascular damage resulting in arterial stiffening like that previously described, but with a more acute onset as a result of concussive injury. It is possible, however, that subconcussive blows experienced over an athletic career result in similar microvascular damage and thus explain why no differences between our concussed and healthy groups was found. Further research is warranted to examine the effect of subconcussive blows on arterial function, however, before this hypothesis is confirmed.

\section{Conclusion}

Concussed and healthy subjects did not differ in measures of arterial stiffness as obtained by the BAM. Also, neurocognitive test scores were not significantly correlated with BAM outcomes, indicating a limited influence of arterial stiffness of neurocognitive function. Given these findings, it seems that current recommendations regarding initiation of a graded return to play protocol once symptoms resolve are sound and continued adherence to these guidelines warranted. 
Table 5. Subject Demographics, Sport Type, and Concussion History for Concussed and Healthy Groups.

\begin{tabular}{lll}
\hline \multirow{2}{*}{ Variable } & Group & Healthy \\
\cline { 2 - 3 } Age (years) & $20.0 \pm 1.4$ & $19.8 \pm 0.9$ \\
Height (cm) & $175.3 \pm 15.2$ & $177.8 \pm 11.9$ \\
Weight (Kg) & $85.4 \pm 31.5$ & $82.1 \pm 22.3$ \\
Gender (M:F) & $10: 6$ & $10: 6$ \\
Sport type: & $10(62.5 \%)$ & \\
$\quad$ Collision & $4(25.0 \%)$ & $9(56.3 \%)$ \\
$\quad$ Contact & $3(12.5 \%)$ & $7(43.8 \%)$ \\
$\quad$ Limited Contact & -- & -- \\
$\quad$ Noncontact & $12(75 \%)$ & $3(18.8 \%)$ \\
Concussion History $(\%)$ & & $13(81.3 \%)$ \\
No. of Past Concussions & $10(62.5 \%)$ & $2(12.5 \%)$ \\
0 (first time) & $3(18.8 \%)$ & -- \\
1 & $2(12.5 \%)$ & $1(6.3 \%)$ \\
2 & $1(6.3 \%)$ & -- \\
3 & $4.5 \pm 3.3$ days & \\
Time of assessment & &
\end{tabular}


Table 6. Concussed and Healthy group means, standard deviations, medians, and interquartile ranges for BAM variables.

\begin{tabular}{lll}
\hline Variable & Mean $\pm \mathrm{SD}$ & Median $\left(1^{\text {st }}, 3^{\text {rd }}\right.$ quartiles $)$ \\
\hline $\begin{array}{l}\text { Left Systolic Interval } \\
\text { Concussed }\end{array}$ & $0.12 \pm 0.05$ & $0.12(0.08,0.14)$ \\
$\quad$ Healthy & $0.11 \pm 0.03$ & $0.11(0.09,0.12)$ \\
Right Systolic Interval & & \\
$\quad$ Concussed & $0.12 \pm 0.04^{*}$ & $0.11(0.10,0.14)^{*}$ \\
$\quad$ Healthy & $0.10 \pm 0.02$ & $0.10(0.08,0.11)$ \\
Left Steepest Rise Gradient & & \\
$\quad$ Concussed & $3.2 \pm 1.6$ & $3.12(1.6,4.8)$ \\
$\quad$ Healthy & $3.8 \pm 2.8$ & $2.86(1.8,4.1)$ \\
Right Steepest Rise Gradient & & \\
$\quad$ Concussed & $3.6 \pm 2.6$ & $2.96(2.0,4.3)$ \\
$\quad$ Healthy & $3.6 \pm 1.5$ & $3.37(2.6,4.2)$ \\
Left Peak Interval & & $0.18(0.12,0.25)$ \\
$\quad$ Concussed & $0.18 \pm 0.07$ & $0.16(0.13,0.24)$ \\
$\quad$ Healthy & $0.17 \pm 0.06$ & \\
Right Peak Interval & & $0.23(0.11,0.25)$ \\
$\quad$ Concussed & $0.20 \pm 0.08$ & $0.21(0.15,0.25)$ \\
$\quad$ Healthy & $0.19 \pm 0.07$ & $14.5(6.0,39.4)$ \\
Left Augmentation Index & & $31.6(10.3,44.5)$ \\
$\quad$ Concussed & $20.6 \pm 18.2$ & $30.1(11.1,50.2)$ \\
$\quad$ Healthy & $29.5 \pm 21.5$ & $23.6(12.3,42.0)$ \\
Right Augmentation Index & $30.0 \pm 21.5$ & \\
$\quad$ Concussed & $27.2 \pm 16.5$ & \\
Healthy & &
\end{tabular}

$*$ indicates significant difference from healthy controls $(\mathrm{p} \leq 0.05)$ 
Table 7. Neurocognitive test scores and SAC, BESS, Total Symptoms, and Symptom Severity for Concussed and Health Group

\begin{tabular}{lllll}
\hline \multirow{2}{*}{ Variable } & \multicolumn{2}{c}{ Concussed } & \multicolumn{2}{c}{ Healthy } \\
\cline { 5 - 5 } Processing Speed & $2.46 \pm 0.3$ & $2.49(2.2,2.7)$ & $2.62 \pm 0.3$ & $2.6(2.4,2.8)$ \\
Complex Reaction Time & $0.76 \pm 0.19$ & $0.72(0.61,0.83)$ & $0.74 \pm 0.08$ & $0.72(0.67,0.84)$ \\
Simple Reaction Time & $0.45 \pm 0.11$ & $0.43(0.38,0.47)$ & $0.39 \pm 0.06$ & $0.39(0.33,0.44)$ \\
SAC & $27.3 \pm 1.5$ & $27.5(26.0,28.0)$ & $27.1 \pm 2.0$ & $27.0(26.0,29.0)$ \\
BESS & $27.0 \pm 2.3$ & $27.5(25.0,29.0)$ & $27.3 \pm 2.1$ & $28.0(26.3,29.0)$ \\
Total Symptoms & $4.3 \pm 7.6$ & $0.5(0.0,7.5)$ & $2.1 \pm 2.9$ & $0.5(0.0,3.0)$ \\
Symptom Severity & $8.3 \pm 15.7$ & $0.5(0.0,12.6)$ & $3.3 \pm 4.1$ & $1.0(0.0,6.0)$
\end{tabular}

SAC: Standardized Assessment of Concussion; BESS: Balance Error Scoring Systom 
Table 8. Spearman's Rho Correlations between Neurocognitive Assessments and BAM Outcomes.

\begin{tabular}{lllllll}
\hline Variable & \multicolumn{2}{c}{ Processing Speed } & \multicolumn{2}{c}{ Complex RT } & \multicolumn{2}{c}{ Simple RT } \\
& Rho & p-value & Rho & p-value & Rho & p-value \\
\hline Left Systolic Interval & 0.03 & 0.86 & $0.33^{*}$ & 0.067 & -0.05 & 0.79 \\
Right Systolic Interval & -0.09 & 0.62 & 0.13 & 0.49 & $-0.32^{*}$ & 0.07 \\
Left Steepest Rise Gradient & 0.24 & 0.19 & 0.23 & 0.21 & 0.07 & 0.71 \\
Right Steepest Rise Gradient & 0.18 & 0.32 & 0.18 & 0.33 & -0.07 & 0.71 \\
Left Peak Interval & $0.38^{*}$ & 0.03 & 0.24 & 0.18 & -0.007 & 0.97 \\
Right Peak Interval & $0.35^{*}$ & 0.05 & 0.21 & 0.26 & -0.07 & 0.71 \\
Left Augmentation Index & $0.34^{*}$ & 0.06 & 0.12 & 0.52 & -0.002 & 0.99 \\
Right Augmentation Index & $0.33^{*}$ & 0.06 & 0.18 & 0.32 & -0.10 & 0.57 \\
\hline
\end{tabular}

*Indicates significant correlation with $\mathrm{p} \leq 0.10$ 


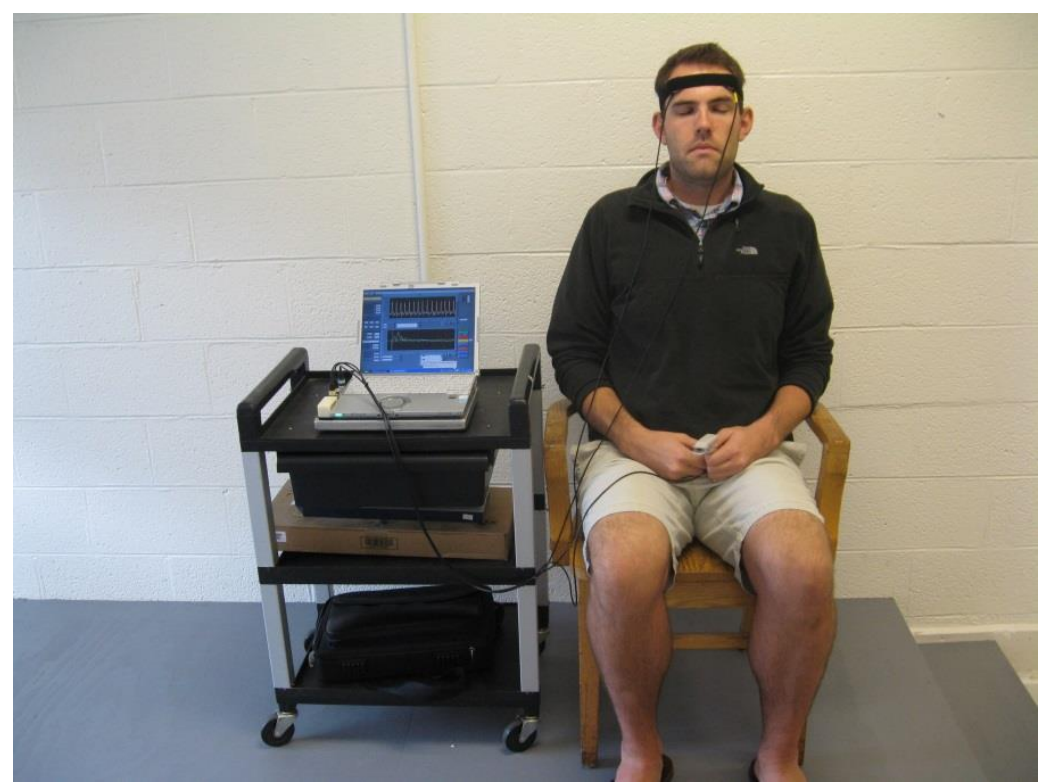

Figure 9. BAM sensors applied to forehead (beneath elastic strap) and thumb.

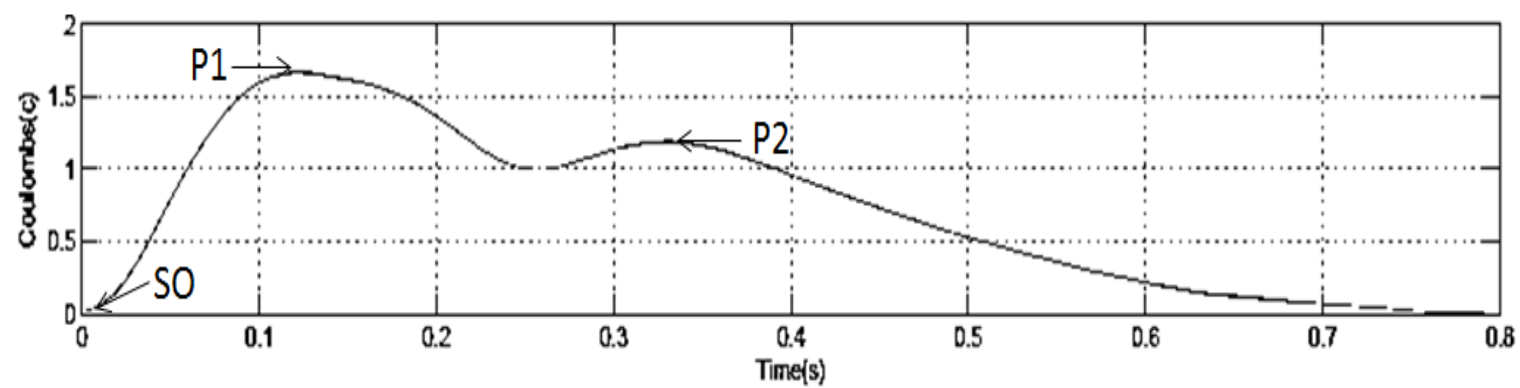

Figure 10. Example arterial pulse wave. $\mathrm{P} 1=$ peak systolic pressure; $\mathrm{P} 2=$ peak of reflected wave; $\mathrm{SO}=$ systolic onset. 


\section{REFERENCES}

1. Langlois JAs, Rutland-Brown W, Wald MA. The epidemiology and impact of traumatic brain injury: A brief overview. Journal of Head Trauma Rehabilitation. 2006;21(5):375-376-378.

2. McCrory P, Johnston K, Meeuwisse WH, et al. Summary and agreement statement of the 2nd international conference on concussion in sport, prague 2004. Br J Sports Med. 2005;39:196-204.

3. McCrory P, Meeuwise W, Johnston K, et al. Consensus statement on concussion in sport - the 3rd international conference on concussion in sport held in zurich, november 2008. Clin J Sports Med. 2009;19(3):185-200.

4. McCrory P, Meeuwisse WH, Aubry M, et al. Consensus statement on concussion in sport: The 4th international conference on concussion in sport held in zurich, november 2012. British journal of sports medicine. 2013;47:250.

5. Guskiewicz KM, Bruce SL, Cantu RC, et al. <br />National athletic trainers' association position statement: Management of sport-related concussion. Journal of athletic training. 2004;9(3):280-281-297.

6. Giza CC, Difiori JP. Pathophysiology of sports-related concussion: An update on basic science and translational research. Sports Health. 2011;3(1):46-51. doi:

$10.1177 / 1941738110391732$.

7. Barkhoudarian G, Hovda DA, Giza CC. The molecular pathophysiology of concussive brain injury. Clin Sports Med. 2011;30:33-48.

8. Werner C, Engelhard K. Pathophysiology of traumatic brain injury. $\mathrm{Br} J$ Anaesthesia. 2007;1:4.

9. Bigler ED, Maxwell WL. Neuropathology of mild traumatic brain injury: Relationship to neuroimaging findings. Brain Imaging Behav. 2012. www.refworks.com. doi: 10.1007/s11682-011-9145-0.

10. Livingston SC, Goodkin HP, Hertel JN, Saliba EN, Barth JT, Ingersoll CD. Differential rates of recovery after acute sport-related concussion: Electrophysiologic, symptomatic, and neurocognitive indices. J Clin Neurophys. 2012;29(1):23.

11. Henry LC, Tremblay S, Leclerc S, et al. Metabolic changes in concussed american football players during the acute and chronic post-injury phases. BMC Neurology. 2011;11:105. 
12. LaMonte MP, Sewell J, Bahouth MN, Sewell C. A noninvasive portable acoustic diagnostic system to differentiate ischemic from hemorrhagic stroke. J Neuroimaging. 2005;15:57.

13. Dutton RP, Prior K, Cohen R, et al. Diagnosing mild traumatic brain injury: Where are we now? $\langle\mathrm{br} />$. Journal of Trauma Injury, Infection, and Critical Care. 2011;70(3):554-555-559.

14. Dutton RP, Sewell J, Aarabi B, Scalea TM. Preliminary trial of a noninvasive brain acoustic monitor in trauma patients with sever closed head injury. Journal of Trauma. 2002;53:857-858-863.

15. Dutton RP, Van der Heijden MS, Aarabi B, Sewell J, Scalea TM. Screening TBI patients with the brain acoustic monitor: Association with CT scan findings and neurologic status at hospital discharge. $\langle b r /\rangle$. Clinical Intensive Care. 2005;16(2):9798-105.

16. Laurent S, Cockcroft J, Van Bortel L, et al. Expert consensus document on arterial stiffness: Methodological issues and clinical applications. European Heart Journal. 2006;27:2588.

17. Shrout PE, Fleiss JL. Intraclass correlations: Uses in assessing rater reliability. Psychological Bulletin. 1979;86(2):420.

18. Atkinson G, Nevill AM. Statistical methods for assessing measurement error (reliability) in variables relevant to sports medicine. Sport Med. 1998;26(4):217.

19. Giza CC, Hovda DA. The neurometabolic cascade of concussion. J Athl Train. 2001;36(3):228.

20. Len TK, Neary P, Asmundson GJG, Goodman DG, Bjornson B, Bhambhani YN. Cerebrovascular reactivity impairment after sports-induced concussion. Medicine \& Science in Sport \& Exercise. 2011:2241.

21. Tegeler C, Kim J, Collins G, et al. Dynamic vascular assessment of brain circulation for sports-related concussion. European Society of Neurosonology and Cerebral Hemodynamics, Wetzlar, Germany. 2004:1-38.

22. Maugans TA, Farley C, Altaye M, Leach J, Cecil KM. Pediatric sports-related concussion produces cerebral blood flow alterations. Pediatrics. 2012;129:28.

23. Bouma GJ, Muizelaar JP. Cerebral blood flow in severe clinical head injury. New Horiz. 1995;3(3):384. 
24. Hlatky R, Contant CF, Diaz-Marchan P, Valadka AB, Robertson CS. Significance of reduced cerebral blood flow during the first 12 hours after traumatic brain injury. Neurocrit Care. 2004;1(1):69.

25. Zwienenberg M, Muizelaar JP. Cerebral perfusion and blood flow in neurotrauma. Neurol Res. 2001;23(2-3):167.

26. Schramm P, Klein KU, Pape M, et al. Serial measurement of static and dynamic cerebrovascular autoregulation after brain injury. J Neurosurg Anesthesiol. 2011;23(1):41-44.

27. Gergont A, Krol-Jawien W, Werynski P, Herman-Sucharska I, Zajac A, Glen A. Results of cerebral angiography performed after neuroradiological indications in children with CNS diseases. Przegl Lek. 2007;64(11):937.

28. Nowak A, Gergont A, Steczkowska M. Assessment of cerebral blood flow after visual stimulation in children with a migraine and chronic tension-type headache-preliminary reports. Prezgl Lek. 2008;65(11):777.

29. Roach ES, Stump DA. Regional cerebral blood flow in childhood headache. Headache. 1989;29(6):379.

30. Duprez DA, Kaiser DR, Whitwam W, et al. Determinants of radial artery pulse wave analysis in asymptomatic individuals. American Journal of Hypertension. 2004;17(8):647.

31. O'Rourke MF, Safar ME. Relationship between aortic stiffening and microvascular disease in brain and kidney. Hypertension. 2005;46:200.

32. Scuteri A, Tesauro M, Appolloni S, Preziosi F, Brancati AM, Volpe M. Arterial stiffness as an independent predictor of longitudinal changes in cognitive function in the older individual. J Hypertension. 2007;25:1035.

33. Nichols WW. Clinical measurement of arterial stiffness obtained from noninvasive pressure waveforms. Am J Hypertens. 2005;18:3S.

34. Junger EC, Ghatan S, Douville CM, Lam AM, Aaslid R, Winn HR. Cerebral autoregulation following minor head injury. N Neurosurg. 1997;86:425-432.

35. Rangel-Castilla L, Gasco J, Nauta HJ, Okonkwo DO, Robertson CS. Cerebral pressure autoregulation in traumatic brain injury. Neurosurg Focus. 2008;25:E7.

36. Strebel S, Lam AM, Matta BF, Newell DW. Impaired cerebral autoregulation after mild brain injury. Surg Neurol. 1997;47:128. 
37. Golding EM, Robertson CS, Bryan RM. The consequences of traumatic brain injury on cerebral blood flow and autoregulation: A review. Clin Exp Hypertens. 1999;21:299.

38. Len TK, Neary JP, Goodman DG, Asmundson GJG. Effect of mild traumatic brain injury on cerebrovascular response. Br J Sports Med. 2009;43 (Suppl):i103-i104.

39. Gall B, Parkhouse W, Goodman D. Heart rate variability of recently concussed athletes at rest and exercise. Med Sci Sports Exerc. 2004;36(8):1269-1274.

40. Oertel M, Boscardin WJ, Obrist WD, et al. Posttraumatic vasospasm: The epidemiology, severity, and time course of an underestimated phenomenon: A prospective study performed in 299 patients. J Neurosurg. 2005;103:812-824.

41. Markus HS. Transcranial doppler ultrasound. British Medical Bulletin. 2000;56(2):378-388.

42. Breedlove EL, Robinson M, Talavage TM, et al. Biomechanical correlates of symptomatic and asymptomatic neurophysiological impairment in high school football. J Biomechanics. 2012;X(XX):XXX.

43. Blumbergs P, Scott G, Manavis J, Wainwright H, Simpson DA, McLean AJ. Staining of amyloid precursor protein to study axonal damage in mild head injury. The Lancet. 194;344(8929):1055.

44. McAllister TW, Saykin AJ, Flashman LA, et al. Brain activation during working memory 1 month after mild traumatic brain injury: A functional MRI study. Neurology. 1999;53:1300.

45. McAllister TW, Sparling MB, Flashman LA, Guerin SJ, Mamourian AC, Sykin AJ. Differential working memory load effects after mild traumatic brain injury. Neuroimage. 2001;14:1004.

46. Vagnozzi R, Signoretti S, Cristofori L, et al. Assessment of metabolic brain damage and recovery following mild traumatic brain injury: A multicentre, proton magnetic resonance spectroscopic study in concussed patients. Brain. 2010:1.

47. Bonne O, Gilboa A, Louzoun Y, et al. Cerebral blood flow in chronic symptomatic mild traumatic brain injury. Pyschiatry Research: Neuroimaging. 2003;124:141.

48. Nilsson B, Ponten U. Experimental head injury in the rat: Regional brain energy metabolism in concussive trauma. J Neurosurg. 1977;47:252. 
49. Kim J, Whyte J, Patel S, et al. Resting cerebral blood flow alterations in chronic traumatic brain injury: An arterial spin labeling perfusion fMRI study. $J$ Neurotrauma. 2010;27:1399.

50. Velard F, Fisher D, Hovda DA. Fluid percussion injury induces prolonged changes in cerebral blood flow. J Neurotrauma. 1992;9:402.

51. Doberstein C, Velarde F, Badie H, Hovda DA. Changes in local cerebral blood flow following concussive brain injury. Society for Neuroscience. January 1, 1992;18:175.

52. Yamakami I, McIntosh T. Effects of traumatic brain injury on regional cerebral blood flow in rats as measured with radiolabeled microspheres. J Cereb Blood Flow Metab. 1989;9:117.

53. Pase MP, Pipingas A, Kras M, et al. Healthy middle-aged individuals are vulnerable to cognitive deficits as a result of increased arterial stiffness. J Hypertension. 2010;28:1724.

54. White NM. Some highlights of research on the effects of caudate nucleus lesions over the past 200 years. Behav Brain Res. 2009;199:3-23.

55. Graybel AM. The basal ganglia: Learning new tricks and loving it. Curr Opin Neurobiol. 2005;15:638-644.

56. Seger CA, Cincotta CM. The roles of the caudate nucleus in human classification learning. J Neurosci. 2005;25:2941 - 2951.

57. Poels MMF, van Oijen M, Mattace-Raso FUS, et al. Arterial stiffness, cognitive decline, and risk of dementia: The rotterdam study. Stroke. 2007;38:888. 


\section{APPENDIX A}

\section{The Problem}

\section{Statement of the Problem}

Current recommendations for diagnosis and management of sport related concussion include patient-reported symptom inventories, computerized neurocognitive testing, and assessment of balance. These tools, while helpful, focus on the functional manifestations of the molecular and metabolic disturbances that result from concussive injury. Recent studies have found metabolic imbalances and reductions in cerebral blood flow beyond resolution of symptoms and neurocognitive function, which are typically the most objective means of determining recovery used by clinicians today. There is a dire need for tools that are easily accessible and able to objectively measure physiological, not just functional, changes associated with concussion. The Brain Acoustic Monitor may offer a solution through examination of arterial pressure waveforms. Concussion and its associated biomechanics result in diffuse shearing forces that may induce microtearing within the cerebral vasculature and, in turn, may lead to increased arterial stiffness. While measurement of this truly physiological phenomenon may be useful, many questions regarding the reliability and ability of the Brain Acoustic Monitor to identify differences must be answered before recommendations regarding its use in a clinical population can be made. 


\section{Research questions}

Manuscript I:

- Are the outcomes associated with the Brain Acoustic Monitor consistent in dayto-day assessments?

- Are the outcomes obtained using the Brain Acoustic Monitor repeatable between different clinicians?

\section{Manuscript II:}

- Do measures of arterial stiffness including systolic interval, steepest rise gradient, peak interval, and augmentation index obtained using the Brain Acoustic Monitor differ in concussed individuals 24 to 48 hours post-injury, at resolution of symptoms, and return-to-activity?

- Do concussed individuals and healthy age, gender, and sport contact matched controls differ in systolic interval, steepest rise gradient, peak interval, and augmentation index at important clinical time points following injury?

Manuscript III:

- Do concussed individuals and healthy, age, gender, and sport contact matched controls differ in systolic interval, steepest rise gradient, peak interval, and augmentation index at resolution of symptoms?

- Are deficits in neurocognitive function correlated with measures of arterial stiffness in healthy and concussed individuals?

- Do any measures of arterial stiffness predict poorer neurocognitive test scores in healthy and concussed individuals? 


\section{Experimental Hypothesis}

Manuscript I:

- Systolic interval, peak interval, steepest rise gradient, and augmentation index will not be significantly different in day 1 and day 2 measures or between assessor 1 and assessor 2.

- BAM outcomes of systolic interval, peak interval, steepest rise gradient, and augmentation index will have moderate reliability.

\section{Manuscript II:}

- Concussed individuals will have increased arterial stiffness, evidenced by decreased systolic interval, peak interval, and augmentation index and increased steepest rise gradient, at 24 to 48 hours compared to symptom resolution and return-to-activity.

- Concussed individuals will have increased arterial stiffness, evidenced by decreased systolic interval, peak interval, and augmentation index and increased steepest rise gradient, at symptom resolution compared to return-to-activity.

- Concussed individuals will have decreased systolic and peak intervals and augmentation index and increased steepest rise gradient compared to healthy controls at 24 to 48 hours post-injury and symptom recovery.

- Concussed individuals and healthy controls will no differ in systolic interval, steepest rise gradient, peak interval, or augmentation index at return-to-activity. 


\section{Manuscript III:}

- Concussed individuals will have lower systolic and peak interval and augmentation index and greater steepest rise gradient compared to healthy controls.

- Systolic Interval, steepest rise gradient, peak interval and augmentation index will be significantly correlated with processing speed, complex reaction time, and simple reaction time.

- Peak interval and augmentation index are significant predictors of processing speed, complex reaction time, and simple reaction time.

\section{Assumptions}

- Team athletic trainers and/or their team physicians diagnosed concussions accurately and all concussed individuals did not suffer from comorbidities.

- Post-concussion symptoms were the result of a concussion and not underlying psychological disorders or other physical disorders such as migraines.

- Concussed individuals will be honest regarding symptom resolution.

- All individuals have provided full effort on baseline and post-injury neurocognitive test scores.

- The neurocognitive tests included in this study measured the outcomes intended (processing speed, complex reaction time, and simple reaction time).

- The BAM outcomes are truly measuring physiological alterations in arterial function and resultant differences in arterial pressure characteristics. 


\section{Delimitations}

- Concussed individuals were included in this study if:

- They were diagnosed by their team athletic trainer or physician

- They experienced a biomechanically induced injury either directly to the head or indirectly to the trunk with forces transferred to the head.

- An initial assessment with the Brain Acoustic Monitor was obtained at symptom resolution or earlier.

- Symptoms were not the result of underlying psychological disorders or physical disorders such as migraines.

- They had undergone baseline neurocognitive testing prior to athletic participation.

- They had no known neurological that might affect balance or cognition

- They had no history of seizures

- Healthy individuals were included if:

- They had undergone baseline neurocognitive testing prior to athletic participation.

○ They had no prior history of concussion within 6 months of baseline neurocognitive testing or BAM assessment.

- They had no known neurological disorders that might affect balance or cognition.

○ They had no history of seizures. 


\section{Limitations}

Manuscript I:

- Caffeine intake, amount of sleep obtained the night prior to testing, and time of day for testing was not controlled between day 1 and day 2 assessments.

- Sensor application was not standardized between day 1 and day 2 sessions nor for inter-rater assessments.

- Comparison of a day-to-day measures may not reflect the true reliability of serial assessments that may be performed multiple times daily or over several days.

- Assessor training, specifically regarding subjective assessment of the raw acoustic signal during testing, was not equal.

Manuscript II:

- No baseline measures were used for within-group comparisons of concussed individuals at 24-48 hours post-injury, symptom resolution, or return-to-play.

- Healthy and concussed individuals were matched by sport contact category (collision, contact, limited contact, or noncontact) rather than individual sport and position.

- Neurocognitive testing was performed using three different computerized testing formats and comparison of these measures is limited. 


\section{Manuscript III:}

- Percent-change for neurocognitive test scores would have provided better assessment of neurocognitive dysfunction than comparison by z-score.

- Length of participation in sport and prior sport involvement was not collected. Similarity of concussion risk and head impact was assumed based on current sport contact category.

- Neurocognitive testing was performed using three different computerized testing formats and comparison of these measures is limited.

\section{Operational Definitions:}

Concussion: diagnosis of concussion was based on various clinical signs and symptoms in accordance with the consensus released by the 2012 International Committee on Concussion in Sport:

"Concussion is a brain injury and is defined as a complex pathophysiological process affecting the brain, induced by biomechanical forces. Several common features that incorporate clinical, pathologic, and biomechanical injury constructs that may be utilized in defining the nature of a concussive injury include:

1. Direct blow to the head, face, neck, or elsewhere on the body with an "impulsive" force transmitted to the head.

2. Rapid onset of short-lived impairment of neurological function that resolves spontaneously. 
3. Graded set of clinical symptoms that may or may not involve loss of consciousness. Resolution of said symptoms typically follows a sequential course."

Arterial stiffness: constriction or vasospasm of the cerebral vasculature as indicated by increased steepest rise gradient and decreased systolic interval, peak interval, and augmentation index (defined below).

Steepest rise gradient: the steepest portion of the left shoulder of the systolic peak (first peak) in the arterial pressure waveform. Increases in this measure ndicate increased pulse wave velocity.

Systolic interval: the time interval between systolic onset and the peak of the systolic wave (first peak). Decreases in the time between these points indicate increased pulse wave velocity and reduced ability of the cerebral arterioles to attenuate pressure.

Peak interval: the time interval between the peak of the systolic wave (first peak) and the peak of the reflected wave (second peak). Decreases in the time between these points, similar to the systolic interval, indicate increased pulse wave velocity and reduced ability of the cerebral arterioles to attenuate pressure.

Augmentation index: represents the magnitude of the reflected wave in proportion to the systolic wave. This measure is calculated by dividing the difference between the magnitude of the systolic and reflected wave peaks by the magnitude of the systolic wave.

Neurocognitive function: cognitive functions associated with specific areas of the brain responsible for immediate and secondary visual and verbal memory and information 
processing. For this study, neurocognitive function was defined by outcomes associated with the Concussion Resolution Index. Outcomes associated with this test used for this study are described below.

Processing Speed: describes how well an individual can recognize and process information (i.e. perceiving, attending/responding to incoming information, motor speed, fine motor coordination, and visual perceptual ability). This was calculated using subtests of the CRI as the mean time per correct item for symbol scanning and animal decoding subtests

Complex Reaction Time: describes how quickly an individual can respond to a complex direction set. This was determined by averaging mean response time for the visual recognition 1 and 2 subtests on the CRI.

Simple Reaction Time: indicates how quickly an individual is able to respond to simple direction sets. This is represented by the mean response time for the reaction time and cued reaction subtests on the CRI.

\section{Significance of the study}

Clinical assessment of concussion currently relies on measures of the functional manifestations of the metabolic and molecular dysfunction that occurs following injury. While this method has been useful and a multifactorial approach is necessary, there is a need for more objective tools for determination of true recovery. If we are able to better define recovery from concussion, it is likely the long term consequences of concussion, such as early onset dementia or chronic traumatic encephalopathy, may be avoided or their severity reduced. If the BAM is proven as a useful tool in the assessment of 
concussion, sports medicine professionals would finally have a direct method of assessing a physiological consequence of concussion and return-to-activity guidelines could be made more effective in reducing re-injury and later life cognitive dysfunction. 


\section{APPENDIX B}

\section{Literature review}

\section{Introduction}

Sports related concussion is of recent interest in sports medicine professions as long-term consequences are slowly being revealed through advanced imaging and postmortem autopsy. ${ }^{1,2}$ With as many as 3.4 million head injuries occurring due to sport participation, there is a great need for accurate and efficient diagnosis of sport-related concussion and more sensitive markers of recovery such that the long term consequences of concussion are minimized as much as possible. ${ }^{3}$ Current recommendations for concussion recognition and management include a multi-factorial approach involving patient-reported symptom checklists, baseline and post-injury neuropsychological assessments, and balance testing. ${ }^{4,5}$ These tools have shown to be effective in recognizing deficits immediately following concussive injury and are typically used to determine when a patient has recovered by ensuring achievement of baseline performance level. However, more recent evidence suggests that there are minute physiological changes still occurring at the molecular level within the brain beyond recovery of neurocognitive function, balance ability, and symptoms. ${ }^{6}$ Sports medicine professionals dealing with sport related concussion would benefit from a tool that assesses a direct physiological change associated with concussion, rather than the functional manifestations that are commonly used to consider a patient "recovered".

One area of physiological function specifically shown to remain altered following concussion well beyond recovery using standard assessment tools is that of cerebral 
blood flow. A new device, called the Brain Acoustic Monitor (BAM), may offer a solution through the amplification and comparison of sound waves created by cerebral arteries and a reference artery in a peripheral limb. ${ }^{7-10}$ Essentially, this device will allow sports medicine professionals to assess cerebral blood flow changes in their patients following concussion, potentially leading to better recognition and more accurate determination of recovery from concussion. This study is designed to assess the ability of the BAM to delineate differences in cerebral blood flow after concussion throughout the recovery processes. This review of literature will seek to summarize current definitions of concussion, incidence of concussion among different populations, modifiable and unmodifiable factors that influence concussion susceptibility, severity, and recovery, and current tools for assessment including clinical and sideline tools, neuroimaging techniques, and the BAM.

\section{Definition of Concussion}

Often the most challenging aspect of concussion diagnosis is recognizing the injury. Many definitions of sport-related concussion have existed throughout the last few decades, each evolving as the body of evidence surrounding the injury has developed. From grading scales to a more individualized approach, there has never been a truly agreed upon definition, leading to a misunderstanding by lay persons of the seriousness of the injury. The most recent definition of concussion and likely the most widely references is that formed by the International Committee on Concussion in Sport. This Committee meets every 4 years to discuss current evidence regarding concussion presentation, assessment, and management and to develop an updated consensus on best 
practices to act as a guideline for sports medicine professionals. At the most recent consensus meeting held in Zurich in 2012, the Committee set forth the following definition of concussion:

"Concussion is a brain injury and is defined as a complex pathophysiological process affecting the brain, induced by biomechanical forces. Several common features that incorporate clinical, pathologic, and biomechanical injury constructs that may be utilized in defining the nature of a concussive injury include:

$>$ Concussion may be caused either by a direct blow to the head, face, neck, or elsewhere on the body with an "impulsive" force transmitted to the head.

Concussion typically results in the rapid onset of short-lived impairment of neurological function that resolves spontaneously. However, in some cases, symptoms and signs may evolve over a number of minutes to hours.

$>$ Concussion may result in neuropathological changes, but the acute clinical symptoms largely reflect a functional disturbance rather than a structural injury, and as such, no abnormality is seen on standard structural neuroimaging studies.

$>$ Concussion results in a graded set of clinical symptoms that may or may not involve loss of consciousness. Resolution of the clinical and cognitive symptoms typically follows a sequential course. However, it is important to note that in some cases symptoms may be prolonged."

This definition deviates from those held in the late 1990s and early 2000s that used a set of guidelines or criteria to categorize concussions based on severity (i.e. mild, moderate, severe or grade I, II, or III). In these guidelines, loss of consciousness, presence of amnesia and duration of post-concussive symptoms were used as indicators of severity. However, since that time, it has been shown that loss of consciousness may only occur in approximately $10 \%$ of concussions and that post-traumatic amnesia is not always a marker of injury severity.(CITE) These findings have led to an individualized approach to concussion assessment with retrospective identification of severity. 
One area of confusion in defining concussion is its interchangeability with the term 'mild traumatic brain injury' or 'mTBI'. According to the American Medical Society for Sports Medicine, all concussions are a type of mTBI, but not all mTBIs are concussions. ${ }^{11}$ Concussions are a subset of mTBIs, on the less-severe end of the brain injury spectrum and are generally self-limited in duration and resolution. ${ }^{11}$ The Zurich statement supports this claim and suggests that although the terms mTBI and concussion are often used interchangeably in the sporting context and particularly in the US literature, others use the term to refer to different injury constructs. ${ }^{4}$ They also noted that the term commotio cerebri is often used in European and other countries. ${ }^{4}$ Finally, the colloquial term “ding” or "bell-ringer", as suggested by the National Athletic Trainers' Association position statement on concussion management, should be avoided. These terms generally carry a connotation that diminishes the importance and seriousness of the injury. 5

\section{Epidemiology}

Estimates of the incidence of sport-related concussion are muddled by the lack of a globally accepted definition in years past as well as an estimated $40 \%$ that go unreported by athletes. For instance, the Centers for Disease Control have suggested approximately 300,000 sport-related concussions per year, but this estimate only took into account instances where loss of consciousness had occurred. ${ }^{12}$ With somewhere between 8 to 20 percent of concussions reporting loss of consciousness, a better estimate

of concussions experienced could likely be between 1.5 to 3.7 million per year. ${ }^{13,14}$ However, to account for the $40 \%$ of concussions that go unreported, true numbers may be 
in excess of 5 million per year.(CITE). It is important for sports medicine professionals to understand where inconsistencies exist in different populations regarding concussion susceptibility. As a result, a number of studies have been undertaken to begin to examine factors that may make athletes more likely to sustain a concussion, such as age, gender, sport type, presence of psychological disorders, and prior history of concussion.

In a study by Marar et al in 2012, using an internet-based data collection tool RIO, a large sample of US high schools reported athlete exposure and injury data for 20 sports during 2008 to 2010. A total of 1936 concussions were reported during 7,780,064 athlete exposures for an overall injury rate of 2.5 per 10,000 AEs. Injury rate was higher in competition (6.4) than practice (1.1). Concussions represented $13.2 \%$ of all reported injuries. Football players had the highest number (47.1\%) followed by girls' soccer (8.2\%), boys wrestling (5.8\%), and girls' basketball (5.5\%). Football had the high rate of concussion (6.4) compared to boys ice hockey (5.4) and boys' lacrosse (4.0). Girls had higher rates (1.7) than boys (1.0). Differences among female and male athletes have been replicated elsewhere ${ }^{15,16}$ and explanations for observed sex differences range from biomechanical differences such as reduced head to ball ratios or weaker neck strength to cultural influences on the male to "play through the pain" and greater level of protection placed on females. ${ }^{17-20}$

Similar results were found in another study comparing RIO and NCAA Injury Surveillance System (ISS) data for the 2005 to 2006 school year in high school and collegiate athletes. ${ }^{21}$ For this time frame, high school athletes sustained 396 concussions, which made up $8.9 \%$ of all injuries reported. This resulted in a concussion injury rate of 
0.23 per 1000 AEs (practice: 0.11 , competition 0.53 ). Again, the majority of concussions resulted from participation in football (40.5\%) followed by girls soccer (21.5\%), boys soccer (15.4\%), and girls' basketball $(9.5 \%)$. The overall rate of concussion was higher in the collegiate sports than their high school counter parts. Collegiate concussion rates (0.43) were higher than high school rates ( 0.23$)$ in both competition and practice, with the exception of baseball and softball. Conversely, concussions comprised a greater proportion of total injuries sustained by high school athletes (8.9\%) than by collegiate athletes (5.8\%) in all sports except volleyball and men's basketball. The higher rate of concussion among collegiate athletes found in this study was contrary to previous studies. ${ }^{22}$ Guskiewicz and colleagues performed a survey-based examination of concussion rates in collegiate and high school football players in $2000 .{ }^{22}$ A letter of inquiry was sent to 580 athletic trainers and a packet with multiple copies of concussion report forms was sent to those who agreed to participate. Athletic trainers were given instructions to complete a concussion report immediately after each concussion sustained by an athlete during the season. The report also requested information on the circumstances of the injury, such as whether the injury occurred in a game or practice, the position played, how long the athlete remained symptomatic, and how long before the athlete was permitted to return to play. Of the 242 schools that participated in the study, 17549 football players were represented. Of those players, $888(5.1 \%)$ sustained at least one concussion. Incidence of concussion per total athletes was significantly higher at the high school (5.6\%) and division III collegiate division (5.5\%) than at division II (4.5\%) and division I collegiate levels (4.4\%). The overall rate of concussions per 1000 athlete- 
exposures was 0.70 , vastly higher than that presented by Gessel et al. in the previously mentioned study, likely due to the limitation of the population to football players only. These findings suggest that the potential for greater playing-time, lower quality protective equipment, and lower skill level found among high school and division III football players could play a role in greater incidence of concussion when compared to division I and II level collegiate athletes. It is possible, however, that the greater incidence of concussion found among collegiate athletes seen by the previously mentioned study by Gessel et al. could be explained by a greater intensity of play while also increasing the number of other injuries sustained thereby lowering the proportion of all injuries that are concussion.

In a study by Castille et al. in 2011, sports related injury and exposure data were collected for nine sports from 2005 to 2010 from 100 nationally representative US high schools using the injury surveillance system RIO. During 2005 to 2010, 2417 concussions were reported of which 2110 were new (87.3\%) and 292 were recurrent (12.1\%). The injury rate for all concussions (regardless of being new or recurrent) was .253 concussions per 1,000 athlete exposures. The injury rate of new concussions was 0.222 per 1,000 AEs while the recurrence rate was .031 per 1,000 AEs. The highest rate of new concussions occurred in football (47.6), girls' soccer (25.8), boys' soccer (17.4) and girls' basketabll (16.7). The highest rates of recurrent concussions occurred in football (6.3), girls' soccer (4.1), girls' basketball (2.9), and wrestling (2.7). Girls had higher rates of concussions overall (20.4), new concussions (17.6), and recurrent concussions (2.8) compared to boys (11.2, 10.1, and 1.1, respectively). These findings 
further document differences among female and male athletes as seen in the study by Gessel et al. and others. It is plain to see that a number of factors play a role in modifying an individual's susceptibility for concussion, and these are not limited to gender, sport type, and age but may also include history of prior concussion, presence of comorbidities such as attention-deficit disorders, learning disorders, migraine, depression or other mental health disorders, and/or sleep disorders. ${ }^{4}$ Unfortunately, the effect of these additional modifiers on concussion incidence has not been examined to-date.

\section{Concussion Biomechanics and Mechanism of Injury}

The biomechanics of head impact has been studied in many settings over the past six decades. Linear and rotational head accelerations have been hypothesized to be the primary risk factor for concussion during impact. Load of the head may be the result of direct impact or inertial loading such as whiplash. ${ }^{23}$ Examples of direct impacts range from helmet-to-helmet contact, striking an opponent's head with a stick, or being struck in the head by a projectile used in the sport. Indirect impacts are more commonly caused by tackling or body-checking and are the result of abruptly stopping an opponent's body from traveling in the direction in which it was headed. ${ }^{23}$ These forces introduce various strain patterns in brain tissue that, if applied over a long enough time, result in diffuse axonal injury. Because the brain is suspended within the skull and surrounded by cerebrospinal fluid, sudden impact may cause translation of the brain within the skull, resulting in what is known as a "coup" or "contrecoup" injury. A "coup" injury occurs when the brain contacts the skull directly under the site of impact with an object or 
opponent. A "contrecoup" injury, on the other hand, occurs on the side of the brain opposite that which was impacted. Regardless of the mechanism by which forces are applied to the brain, the end result is that the body is unable to overcome the acceleration or deceleration forces that have set it in motion, resulting in some compression and/or shearing of the brain tissue.

\section{Mechanism of Injury by Sport and Position}

Many studies have been performed to determine which mechanisms result in concussion most often and how these differ by sport, gender, and age group. Gessel et al. found the highest proportion of concussion injuries in high school football to occur during running plays and contact with another person, specifically via tackling and being tackled, at $67.6 \%$ of all concussions. ${ }^{21}$ Linebackers suffered $40.9 \%$ of all concussions among defensive players and concussions represented a higher proportion of their total injuries (13.1\%) than for all other defensive positions combined (10.8\%). Runningbacks sustained $29.4 \%$ of concussions suffered by players in offensive positions. In high school soccer, the activity most frequently associated with concussion was heading the ball (40.5\% in boys, $36.7 \%$ in girls) and concussions represented $64.1 \%$ of injuries sustained while heading a ball. Contact with another person resulted in a greater proportion of concussions in boys $(85.3 \%)$ than in girls $(58.3 \%)$, potentially due to the difference in skill and speed found between male and female soccer players. Contact with the ground ( $22.6 \%$ in boys, $5.6 \%$ in girls) and contact with the ball (18.3\% in boys, $8.2 \%$ in girls) were related to a significantly greater proportion of concussions in girls than boys. 
Regarding positions, concussions represented a larger proportion of injuries to goal keepers $(21.7 \%)$ than all other positions $(11.1 \%)$.

These results have been supported by others. ${ }^{24,25}$ Castile et al. examined the rates and mechanisms of first and recurrent concussions among high school athletes across 9 sports and found that the majority of new and recurrent concussions resulted from contact with another person $(73.4 \%$ and $77.9 \%$, respectively). In similarity with the results presented by Gessel et al., they also found a higher proportion of concussions among boys resulting from player-to-player contact than among girls. Interestingly, girls had a higher proportion of first time concussion resulting from contact with the playing surface but not for recurrent concussions. These findings further outline the gender differences not only inherent between males and females via hormonal and biomechanical make-up but also in playing styles and willingness to report an injury. It has been shown that nearly half of high school football players who sustain a concussion are reluctant to report a concussive injury, with the most common reasons being that they did not think it was serious enough to report or they did not want to let down their teammates or coaches, indicating the vitality of proper education of student-athletes on concussion recognition and consequences of continuing to play. ${ }^{26}$

Comparison of Head Impacts by Sport, Gender, and Age

While an exact threshold for injury using true brain dynamics has yet to be determined presently, efforts have been put in place to determine relationships between head acceleration and injury severity and/or types of symptoms caused. (Cite) In sportrelated activity, there is usually some combination of both linear and rotational 
accelerations associated with direct and indirect impacts and many factors are thought to play in an individual's ability to dissipate forces. Some of these factors include differences in cerebrospinal fluid levels and function, vulnerability to brain tissue injury, relative musculoskeletal strengths and weaknesses, and the anticipation of an oncoming direct or indirect impact. ${ }^{23}$ Animal research in the area of linear and rotational acceleration suggests that he latter of these forces is typically more significant and can lead to more serious effects on the brain. ${ }^{27}$ Ommaya and Gennarelli were among the first to describe in detail linear and rotational accelerative mechanisms of injury using animal models that helped to better explain the role of linear versus rotational acceleration for brain injury. ${ }^{27}$ As such, the National Football League commissioned a study of videorecorded concussions, recreating each mechanism of injury using helmeted Hybrid II dummies to examine injury thresholds for sustaining a concussion based on linear acceleration experienced by football players' heads. (add more from NFL study here).

In a study examining how collision-type and player anticipation affected severity of head impact in youth ice hockey, it seems that open-ice collisions resulted in greater linear and rotational accelerations, compared with collisions along the playing boards. ${ }^{28}$ Additionally, anticipated collisions tended to result in less-severe head impacts than unanticipated collisions, especially for medium-intensity impacts. No statistically significant differences existed in linear acceleration among the anticipation types, however, they tended to be highest for unanticipated collisions followed by anticipated impacts with poor positioning and anticipated collisions with good positioning. Similar results were seen for rotational acceleration. These results suggest that severe impacts 
(top 25\% linear acceleration) may be equally dangerous regardless of level of anticipation and intervention strategies employed by coaches, athletic trainers, and other sports medicine professionals should be to target education of players on how to deliver and receive body collisions safely in all areas of the ice, including on the playing boards and on the open ice.

A similar study aimed to measure the magnitude of head impacts sustained by 14 male youth ice hockey players and to compare impacts across event types, player positions, and location of impact. ${ }^{29}$ Game impact magnitudes were significantly greater than practice impacts and more severe impacts were 4.25 times more likely to occur in games than practices. Impacts sustained by forwards were similar in magnitude to those sustained by defensemen. Significant differences were found for head impact locations in that those experienced at the top of the head were greater than those at the back, front, and right sides. Among this sample, players were 5.57 and 4.88 times more likely to sustain an impact greater than $80 \mathrm{~g}$ to the top of the head than on the right and left sides, respectively. Similarly, they were 3.25 and 3.00 times more likely to sustain higher impacts to the top of the head than to the front and back, respectively. The average impact across all players was 20g, similar to those measures in American football players with 14 impacts sustained, on average, during practice and 71 during games.

This trend in a greater frequency of game impacts compared to practice impacts is in contrast to a study of similar design examining Division I collegiate football players. ${ }^{30}$ In this study, head accelerations among 72 college football players were recorded for all practices and games for two competitive season. ${ }^{30}$ These researchers found consistently 
sustained impacts between $21 \mathrm{~g}$ and $23 \mathrm{~g}$. Impacts sustained during helmet-only practices were significantly greater than those sustained in games or scrimmages. Similar to the results of the study previously described using youth hockey players, they found a greater likelihood of impacts to the top of the head compared to those to the front, back, left, and right sides. These authors also examined differences in head impacts among player positions (Table B1). Offensive backs were 1.52, 1.41, 1.24, 1.17, and 1.03 times more likely to sustain an impact of greater than $80 \mathrm{~g}$ than defensive linemen, defensive backs, offensive linemen, linebackers, and wide receivers, respectively. Contrary to the data presented in the youth ice hockey study, this study revealed a greater percentage of impacts during full-contact (50.1\%) and helmet-only (27.2\%) practices in Division I collegiate players than games and scrimmages (22.5\%). These findings were suggested to be likely due to the disparity between practice schedules between collegiate football and youth ice hockey. Collegiate football teams practice almost daily with one game per week over a 13-week period whereas in youth ice hockey the practice to game ratio is closer to one.

Table B1. Frequency of head impacts sustained by position in 2005 and 2006 football seasons.

\begin{tabular}{|c|c|c|}
\hline Position & Frequency of Impacts & Mean $( \pm S D)$ linear acceleration $(g)$ \\
\hline Offensive linemen & $20256(35.52 \%)$ & $22.89 \pm 1.79$ \\
\hline Offensive backs & $7066(12.39 \%)$ & $22.93 \pm 1.83$ \\
\hline Defensive linemen & $12540(21.99 \%)$ & $21.56 \pm 1.76$ \\
\hline Defensive backs & $8767(15.37 \%)$ & $21.02 \pm 1.78$ \\
\hline Linebackers & $5892(10.33 \%)$ & $22.67 \pm 1.81$ \\
\hline Wide receivers & $2503(4.39 \%)$ & $22.19 \pm 1.83$ \\
\hline Total & 57024 & $22.25 \pm 1.79$ \\
\hline
\end{tabular}

**Data from: Mihalik et al. Measurement of head impact in collegiate football players: an investigation of positional and event-type differences. 2007 Neurosurgery, 61:1229-1235 
Another interesting finding in this study is the fact that the head impact frequencies by position do not correlate with the concussion incidence rates seen at each position. ${ }^{30}$ For instance, Guskiewicz et al. reported the lowest concussion rates in running backs and wide receivers compared with linebackers, offensive linemen, linebackers, and wide receivers, respectively. ${ }^{31}$ However, the head impact results presented among the Division I football study suggests, based on frequency of impacts, that offensive backs and wide receivers are more likely to sustain a high magnitude impact than the other positions. While this would logically suggest an increased risk for concussive injury, this was not evidenced in true rate of concussion among these player positions. It is possible that a cumulative effect of repeated low-magnitude impacts is related to an increase in concussion injury rates, leading to the findings of Guskiewicz et al. showing greater incidence of concussion among linebackers, offensive linemen, defensive backs, and defensive linemen. ${ }^{31}$

It is important to understand the different demands of high school and collegiate level football regarding average and peak impacts sustained throughout a season so that true injury susceptibility and long term consequences of said impacts might be understood. Schnebel et al. compared the frequency and magnitude of head impacts between NCAA Division I football players and high school football players. ${ }^{32}$ They found that collegiate athletes sustained high level impacts greater than $98 \mathrm{~g}$ more frequently than high school players. Skill position players received $24.6 \%$ of all impacts and a hit greater than $98 \mathrm{~g}$ was sustained every 70 impacts. Linemen sustained the highest number of impacts but most were relatively low-magnitude at 20-30g. Given the higher 
incidence of concussion in linemen compared to skill players previously presented, this further supports the idea that repeated subconcussive blows do, in fact, play a role in decreasing the threshold for concussion. It is of note, also, that the highest level impacts sustained at the high school level were both frequent ( $0.7 \%$ of all impacts compared to $1.0 \%$ of impacts sustained by collegiate athletes) and at levels greater than $100 \mathrm{~g}$, further supporting the epidemiological data showing that concussion rates are significant at both the high school and college levels.

\section{Associations between mechanisms and injury severity}

Concussive injury presents with different levels of symptom severity. One explanation for this variation is the different biomechanical forces involved in concussive injuries and the heterogeneity of potential mechanisms of injury. With this idea in mind, Guskiewicz and colleagues performed a prospective study using embedded sensors in the helmets of 88 collegiate football players to determine the relationship between recorded head accelerations and impact locations and acute clinical symptomology,

neuropsychological performance, and balance testing. ${ }^{33}$ They recorded hits for 13 concussions during the season with impacts ranging from $60.51 \mathrm{~g}$ to $168.71 \mathrm{~g}$. They found no significant relationships between impact magnitude or impact location and change scores for symptom severity, postural stability, or neurocognitive function. They proposed that the lack of relationship was due to the influence of repetitive subconcussive blows and prior history of concussion. They did, however, note that nearly half of the concussions examined resulted from impacts to the top of the head and presented with some of the greater deficits in postural stability, suggesting that top-of-helmet impacts 
may result in higher rate of concussion than other locations and greater disturbance in balance. While these results are speculative and not statistically based, potential explanations include disruption in the axonal pathways responsible for controlling postural stability (i.e. the cerebellum). ${ }^{33}$ The positioning of the cerebellum between the cerebellar tentorium and the foramen magnum could expose it to impulsive forces as they are directed through the crown of the head. Additionally the authors suggested that the brain centers responsible for central integration of vestibular, visual, and somatosensory information may be impaired.

Similarly, Duhaime and colleagues studied 48 concussions in 44 athletes to analyze the spectrum of clinical presentations used by team medical personnel for diagnosis. ${ }^{34}$ They found that many players had delayed onset of symptoms, the majority of players had delayed diagnosis (hours to days post-injury), more than a third of diagnosed concussions were not associated with a specific impact event, and there was large variability in the levels of linear and rotational acceleration experienced by each concussed athlete. These findings may raise questions not only about the true ability to establish head impact thresholds, but also about differences in diagnosis of concussion.

Interestingly, the idea of head-down or impact to top-of-head impact resulting in more frequent concussion does not seem to hold true for striking players. Viano and collegues analyzed collision mechanics between striking and struck players, the latter becoming concussed, and found that the key to executing a concussive blow is the headdown position. ${ }^{35}$ Because this positioning puts the torso, neck, and head into alignment, the force transmitted to the struck player is maximized, resulting in a $67 \%$ increase in 
mass of the striking player. One possibility for this finding is that knowledge of the strike increased dynamic stability of the players' necks prior to the hit, allowing greater forces to be transmitted through the spine and trunk that if the hit were unsuspected. This role of neck strength in concussion biomechanics has been of interest in explaining sex differences in concussion rates in similar sports as well as prevention of concussions.

In a study examining the role of neck strength in stabilizing and reducing head acceleration during soccer heading, symmetrical strength between neck flexors and extensors appeared to reduce head acceleration during low-velocity laboratory-controlled heading maneuvers. ${ }^{36}$ This suggests that neck strength, due to the ease with which it can be measured and influenced, is a modifiable risk factor for concussive injury. However, no studies to-date have prospectively studied differences in rates of concussions in individuals at each end of the spectrum and, while one could propose that female athletes may represent this cohort, the described study used equal numbers of male and female soccer players and no significant differences in neck flexion or extension strength or their mean imbalance existed between sexes. It is unknown if these findings would be replicated in younger athletes or how neck strength or imbalances between neck flexors and extensors may be implicated in unanticipated hits seen often during other contact sports such as football, ice hockey, and lacrosse.

Studies have been performed to examine the effect of cervical muscle strength on head impact biomechanics and inferences can be made concerning the related influence on concussion risk. In adolescent ice hockey players equipped with instrumented helmets, linear and rotational acceleration was not different between individuals with high, 
moderate, and low strength. While dynamic stability is implicated in the biomechanics of concussion, the methodology used in this study were static tests of isometric strength and the true applicability of these findings to concussion mechanisms is not clear.

\section{Pathophysiology of concussion}

Concussions are often referred to structurally as diffuse axonal injuries and result in some degree of functional impairment but differ from more moderate and severe TBI in that the impairment is transient in nature. ${ }^{23}$ Diffuse axonal injury, in addition to coup and contrecoup mechanism of injury can result in disruption in centers of the brain responsible for breathing, heart rate, and consciousness, but more typically result in memory loss, cognitive deficits, balance disturbances, and a host of other somatic symptoms. More specifically, a "neurometabolic cascade" of events occurs involving various metabolic and ionic shifts in the neuronal functioning in the brain.

Initially, diffuse axonal injury causes a widespread depolarization and subsequent release of excitatory amino acids, particularly glutamate. This release creates an efflux of potassium through various ionic channels. ${ }^{37}$ To restore ionic balance, ATP-dependent sodium-potassium pumps are activated and large stores of metabolized glucose are used. ${ }^{38,39}$ Resultantly, ionic pump activation reduces cellular energy stores and causes neurons to work overtime via anaerobic means of glycolysis to create energy for sodiumpotassium pump activity. This increase in glucose metabolism occurs immediately and advanced imaging techniques suggest it can last hours after injury followed by a period of hypometabolism. ${ }^{40}$ Unfortunately, the by-product of anaerobic glycolysis, lactate, accumulates in the cell and leads to acidosis, increased membrane permeability, and 
cerebral edema, largely reducing mitochondrial function. ${ }^{41}$ This creates a mismatch between the brain's requirement of energy to stabilize ionic imbalances and the inability of the mitochondria to adequately provide it. Couple these events with decreased cerebral blood flow, and the stage is set for more severe brain injury after repeated concussion, also known as second impact syndrome.

Such metabolic abnormalities have historically been based on studies in animals but have more recently been documented in concussed athletes using advanced imaging techniques. ${ }^{42,43}$ Thirteen athletes who sustained concussions were studied using magnetic resonance spectroscopy, which identifies varying concentrations of multiple substrates used for neuronal function such as n-acetyl-aspartate, creatine, lactate, glutamate, and choline. The n-acetyl-aspartate to creatine ratio of injured patients was compared to agematched controls at various clinical time points post-injury. At 3 days post-injury, this ratio was diminished by $18.5 \%$ and did not return to baseline at 15 days. These findings were supported by a similar study ${ }^{42}$ and are important when considering return to activity because the remaining metabolic abnormalities could predispose athletes to further or more severe injury. They may also increase risk of later-life cognitive dysfunction. An important component of the theory behind the current study and the need for better markers of recovery rely largely on research indicating prolonged recovery of these metabolic and ionic imbalances in the presence of recovered symptomology and neurocognitive function.

\section{Cerebral Blood Flow}

Normal cerebral blood flow regulation, relevant anatomy 
The vascular supply to the brain is derived from two pairs of cervical vessels: the internal carotid arteries and the vertebral arteries. The internal carotids supply blood to the anterior intracranial circulation, including the frontal, parietal, anterior and lateral temporal lobes, and the deep gray structures except the thalamus. ${ }^{44}$ The vertebral arteries supply the posterior circulation and these systems are interconnected via anastomoses, namely the Circle of Willis, made up by the anterior and posterior communicating arteries. A portion of the internal carotid, called the carotid bulb or sinus, is responsible for arterial blood pressure regulation. ${ }^{44}$ Raised arterial pressure and increased wall tension within the sinus triggers reflexive bradycardia via the vagus nerve and consequent reduction in blood pressure, assisting in what is known as cerebral autoregulation, or maintenance of constant cerebral perfusion pressure in the presence of alterations in systemic blood pressure.

Once within the cranium, the internal carotid artery will first give rise to the posterior communicating artery and terminate by bifurcating into the middle and anterior cerebral arteries. ${ }^{44,45}$ The middle cerebral artery supplies the largest portion of the cerebral hemispheres including important cortical regions such as the primary sensory and motor cortices, Broca's area, Wernicke's area, and the angular and superior temporal gyri. The anterior and posterior circulation, as previously mentioned, are interconnected via communicating arteries to form a circular anastomosis. This network provides important collateral sources of blood flow so that if three of the four cervical vessels are occluded, the entire brain can be supplied from the remaining vessel. ${ }^{46}$ There are some variants in the Circle of Willis as it is only entirely complete in approximately $25 \%$ of 
individuals. ${ }^{45}$ The most common variants involve an absent posterior communicating artery unilaterally or bilaterally or the presence of a large posterior communicating artery that becomes a branch of the internal carotid rather than the basilar artery. ${ }^{46}$

The control of blood flow in the brain is accomplished by alterations in cerebral perfusion pressure and cerebrovascular resistance. ${ }^{47-49}$ Two main mediating factors that influence these responses include alterations in systemic blood pressure and changes in metabolic demand and byproduct. As previously mentioned, the brain has an intrinsic ability to maintain a constant level of flow in the presence of mean arterial blood pressure, termed cerebral autoregulation. Through mean arterial pressures ranging from approximately $50 \mathrm{mmHg}$ to $175 \mathrm{mmHg}$, cerebral blood flow is maintained around a value of 50 to $60 \mathrm{ml} / 100 \mathrm{~g} / \mathrm{min}^{49}$ Beyond the upper and lower limits of mean arterial pressure, there is a critical decrease or increase in cerebral blood flow that can lead to ischemia or intracranial hemorrhage. The constriction and dilation of cerebral blood vessels to allow for this control occurs primarily in the arteriole and capillary beds. Specifically, by the time blood reaches the small arteries within the pia mater of the meninges, pressure is reduced by 50 to $60 \%$ of systemic pressure. ${ }^{50}$ Because the small vessels in the brain are constantly perfused, this reduction in pressure aids in prevention of microvascular damage.

Cerebrovascular reactivity, or the ability of the cerebral vessels to dilate and constrict in response to alterations in metabolic byproducts, particularly carbon dioxide, is another important regulatory factor in blood flow of the brain. Increases or decreases in the arterial partial pressure of carbon dioxide $\left(\mathrm{PaCO}_{2}\right)$ typically result in rapid increases 
or decreases in cerebral blood flow, respectively. Up to a 5\% increase in blood flow of the cerebrum has been cited following $1 \mathrm{mmHg}$ increase in the $\mathrm{PaCO}_{2}{ }^{47,51,52}$ The normal range of $\mathrm{PaCO}_{2}$ is $35-45 \mathrm{mmHg}$ with hypocapnia being defined as $\mathrm{PaCO}_{2}$ less than 35 $\mathrm{mmHg}$ and hypercapnia greater than $45 \mathrm{mmHg} .{ }^{47}$ Cerebral blood flow increases with hypercapnia to wash out $\mathrm{CO} 2$ from brain tissue, attenuating a rise in central $\mathrm{PaCO} 2$. Alternatively, hypocapnia causes cerebral vasoconstriction, reducing cerebral blood flow. The brain relies on these mechanisms to prevent acidosis. ${ }^{53-55}$

\section{Effect of Concussion on Cerebral Blood Flow}

The effects concussion on of cerebral blood flow are not well studied, however some inferences can be made via studies in severe traumatic brain injury. It has been shown that there is a triphasic response to severe TBI post-injury. Immediately, cerebral hypoperfusion occurs with an average cerebral flow of $32.3 \mathrm{ml} / 100 \mathrm{~g} / \mathrm{min}$. On days 1 to 3 post-injury, cerebral hyperemia occurs with an average of $46.8 \mathrm{ml} / 100 \mathrm{~g} / \mathrm{min}$ and elevated cerebral artery velocities at $89 \mathrm{~cm} / \mathrm{s}^{37}$ During post-injury days 4 to 15 , cerebral vasospasm is thought to occur with reductions in flow to $35.7 \mathrm{ml} / 100 \mathrm{~g} / \mathrm{min}$ and elevated flow velocity of $96.7 \mathrm{~cm} / \mathrm{s}^{37}$ A similar response from concussion is likely based on the fact that there are similar, yet reduced, ionic and metabolic shifts after concussion. However, results from recent studies are inconsistent, with heightened responses in some cases while in others no changes are seen. ${ }^{56-63}$

Studies have shown more definitive dysfunction regarding concussed individuals' ability to autoregulate cerebral blood flow. ${ }^{51,64-66}$ It is suggested that following head injury, the lower shoulder of the autoregulatory curve is shifted to the right such that 
injured patients are less able to deal with lowered cerebral perfusion pressure, increasing their susceptibility for ischemic injury. While this dysfunction appears to recover in days following injury, autoregulation has been shown to be lost or impaired nearly 2 weeks following injury in some studies. In particular, Junger assessed 29 patients who suffered an mTBI with an associated Glascow Coma Scale score of 13 to 15 upon arrival to the emergency room. ${ }^{57}$ Blood flow velocity in all subjects were assessed within 48 hours of injury using transcranial Doppler ultrasound following introduction of systemic blood flow alterations using large blood pressure cuffs placed around each thigh. The authors found a reduced response in eight of their mTBI patients and this difference was correlated with reduced cerebral perfusion pressure.

Other studies have found similar differences using different techniques with no environmental changes induced. For instance, Maugans and colleages studied twelve children and age and gender matched controls between the ages of 11 and 15 using computerized neurocognitive testing, traditional MRI, and more advanced imaging techniques such as susceptibility weighted imaging, diffusion tensor imaging, magnetic resonance imaging, and phase contrast angiography ${ }^{67}$ The investigators found no differences in traditional MRI, diffusion tensor imaging, or magnetic resonance spectroscopy. Neurocognitive testing showed significantly higher symptoms and prolonged reaction time at 72 hours post-injury. At two weeks, symptomology had recovered between groups, but reaction time was still significantly higher in the concussed group. This was paralleled with a reduction in blood flow (using phase contrast angiography) among $73 \%$ of the concussed individuals. This reduction in blood 
flow persisted at 1 month follow-up when neurocognitive test scores had returned to control values. Only $64 \%$ of the concussed group showed recovery to within $10 \%$ of control values at this follow-up assessment. These results were replicated in a study that assessed blood flow in mTBI patients who were still symptomatic a minimum of 2 years following injury. ${ }^{61}$ Blood flow was assessed using brain SPECT. Relative reductions in blood perfusion in head injured individuals compared to healthy controls was seen particularly in the medial and lower aspects of the temporal and frontal regions. The differences found in this sample also correlated moderately with neurocognitive deficits identified during a neuropsychological assessment, suggesting a potential role of cerebral blood flow deficit in complicated recovery from head injury.

This idea of reduced blood flow in areas of the brain corresponding to identified neurocognitive deficits has been supported elsewhere. In a study by Ge and colleagues in 2009, patients with mTBI demonstrated lower cerebral blood flow using arterial spin labeling than normal controls in both sides of the thalamus, which was significantly correlated with several neurocognitive measures including processing and response speed, memory/learning, verbal fluency, and executive function. ${ }^{68}$ Thalamic injury has been largely ignored in prior concussion and mTBI-focused research; however, these findings warrant further research on the role of thalamic dysfunction in post-concussion symptomology and recovery, particularly due to its role as the central relay station that participates in communication among sensory, motor, and associative brain regions.

Other theories regarding alteration in cerebral blood flow involve dysfunction in the autonomic control of cerebral arterial tone, particularly in response to alterations in 
the partial arterial pressure of carbon dioxide $\left(\mathrm{PACO}_{2}\right)$. Len and colleagues examined the cerebrovascular reactivity of 10 concussed individuals an average of 4.5 days postconcussion. ${ }^{54}$ Using 5 bouts of 20 -second breath holding and hyperventilation to induce hyper- and hypocapnia, respectively, cerebral blood flow response was examined using transcranial Doppler ultrasound. Percent-change in the blood flow velocity of the middle cerebral artery was significantly lower in the recovery period of the second hyperventilation of the concussed group and they failed to return to resting levels after all bouts of breath holding. The authors purported these findings to be the result of an uncoupling between the autonomic and cardiovascular system. They also support prior studies examining heart-rate variability among concussed individuals with resolved postconcussion signs and symptoms. Gall et al. measured the heart rate variability in junior ice hockey players and healthy controls at rest and following a bout of submaximal exercise. ${ }^{6}$ At rest, heart rate variability appeared normal compared to controls. However, once exposed to submaximal exercise, abnormal heart rate variability was seen, further evidencing the idea of dysfunction of autonomic control of cardiovascular function. There has also been research that suggests differing cerebrovascular responses to concussion or mTBI based on age. A recent study found reduced cerebrovascular reactivity in younger subjects (less than 30 years old) and no change post-injury in older subjects (greater than 30 years old). The authors of this study suggested that this difference may be connected to the altered activity of the autonomic nervous system in younger individuals. 
Cerebral oxygenation, or lack thereof, following mTBI or concussion has also shown to predict prolonged outcomes and may be an important factor to consider in assessing cerebral blood flow post-concussion. Results using near-infrared spectroscopy have shown that cerebral oxygenation is reduced by up to $35 \%$ on the day of injury and deficits appear to persist up to 7 days following the mTBI. ${ }^{69}$ Additionally, studies using functional MRI have shown that concussed individuals have different brain activation responses than control subjects and that those with higher activation patterns require a longer recovery period. ${ }^{70-73}$

\section{The Brain Acoustic Monitor}

The Brain Acoustic Monitor (BAM) is a noninvasive device purported to detect changes in cerebral arterial function. This portable device detects, amplifies, and displays sound waves that are emitted through predictable deformations in the skull in response to each arterial pulse. ${ }^{7}$ The BAM measures and displays the amplitude and frequency distributions of these intracranial pulse signals from either side of the skull and compares them to a reference arterial signal (typically a digital artery). It consists of two $2-\mathrm{cm}$ circular sensors that are held tightly against the skin of the forehead using an elastic band, connected to a signal conditioning box and laptop computer. The two sensors provide broader monitoring throughout the skull and are not specific to perfusion characteristics of each hemisphere of the brain. That is, they permit a stereophonic representation of the acoustical signal from two distinct areas. A single BAM recording requires 10-20 seconds of monitoring time and less than 10 minutes of operator effort. 
The quality of the BAM signal is determined by the magnitude of the arterial wave within the skull and the transmission characteristics of the intervening brain tissue. ${ }^{8}$ In the initial stages of signal processing development, it was thought that the acoustic signals emitted by a healthy brain, as is received by the forehead sensors, would be similar to those emitted from a peripheral artery; whereas in an injured brain, the acoustic signals would sporadic and would not correlate with the pulse waves given by a peripheral artery. ${ }^{7-9,74}$ As such, early studies with the BAM used two principal metrics for numerical assessment. The first, ratio, is measured directly from the time domain signal (raw data) and the second, divergence, is measured from the frequency responses given by each brain sensor and a control (digital arterial) sensor. Ratio was an initial crude assessment of the erraticism of the waveform by calculating the peak excursion above the mean line divided by the peak excursion below the mean. Physiologically, the significance of ratio is found in the idea that when the arterioles in the brain are experiencing vasospasm, the ratio falls below two-indicating an increase in the reflected wave amplitude. Divergence was defined as the maximum deviation of the brain signal's relative frequency above $0 \mathrm{~dB}$. This outcome essentially provided a comparison of each brain signal to the reference or control signal measured at the radial or a digital artery. It is theorized that alterations in the acoustic signals associated with the brain signals after injury, resulting from stiffening of the arterioles in the cerebrum, will be reflected in changes in the frequency distribution. The BAM software has a built-in algorithm that determines the difference between the frequency distributions obtained by the reference sensor and each brain sensor, which is most often below $10 \mathrm{~dB}$ in healthy individuals. 
Having been developed at the R Adams Cowley Shock Trauma Center of the University of Maryland, the BAM has been studied in trauma settings most often in a severely head injured patient population. Using previously established thresholds for "healthy" individuals - indicated by a ratio of greater than $2 \mathrm{~V}$ and a divergence of less than $10 \mathrm{~dB}$. studies of severe traumatic brain injury have found high correlations $(\mathrm{r}=$ 0.81 ) between "normal" BAM values and outcomes at discharge. More specifically, the first study looking at the BAM as a noninvasive measure for prediction of injury severity found erroneous prediction of a poor status in only 3 of 18 cases (positive prediction value of $83 \%$ ) and erroneous prediction of a good outcome in 0 of 10 (positive prediction value of $100 \%) .{ }^{8}$ In this study, initial BAM recording predicted clinical status at discharge in 25 of 28 cases and a normal signal was not found in any patient who went on to a poor outcome. ${ }^{8}$

A follow-up to this study was performed to assess the correlation between the BAM signal measured early after admission for traumatic brain injury and subsequent anatomic and functional evidence of traumatic brain injury on cranial $\mathrm{CT}^{74}$ In this study, 206 traumatic brain injury patients were assessed and characterized as "normal" or "abnormal" and correlations calculated in accordance with findings on cranial CT. Abnormal BAM signal had a sensitivity of $93 \%$ for the presence of any abnormality on CT scan, with no injuries necessitating active intervention being missed by false negatives. The specificity, however, was only $14 \%$ as a large number of false-positives were recorded. When compared to outcomes using the Glascow Outcomes Score, the BAM was highly sensitive with $100 \%$ of patients with a normal signal discharged with a 
GOS of 4 or 5 (moderate or no disability) and $26 \%$ of patients with an abnormal BAM reading had a GOS of less than 4 (severe disability, vegetative, or death).

A study recently performed in an emergency department setting revealed an associated $100 \%$ sensitivity and $30.06 \%$ specificity for the BAM when determining presence of abnormality on CT scan following mild traumatic brain injury. ${ }^{7}$ This study also showed that the BAM correctly classified 163 of 228 mTBI patients, revealing $71 \%$ sensitivity and 30\% specificity. While it seems that these findings may have positive implications for assessing sport-related concussion, it is likely that the severity of injuries that present to the emergency department is greater than the typical case of concussion experienced and assessed by athletic trainers. In particular, it may be that the thresholds are not sensitive enough to truly assess milder cases of mTBI.

As such, recent developments have been made in the signal processing techniques that allow re-calculation of the BAM signal while accounting for an inherent charge leakage associated with piezoelectric forms of acoustic signal acquisition. Using a custom transformation algorithm, the BAM signal can be processed so that measures of arterial stiffness can be obtained. This allows for a more intuitive and physiologically based measurement of arterial function that capitalizes on metrics of an arterial pressure waveform to determine changes in the responsiveness and stiffness of the cerebrovasculature.

\section{Arterial stiffness}

Normal arterial function throughout the body is designed such that pulsatile energy expended with each systolic pulse is restricted to major arteries and absorbed in 
these as a result of arterial wall viscoelasticity. ${ }^{50}$ The renal, central, and coronary beds, however, are different to all others. They receive relatively constant flow at rest to filter blood, sustain, sensitive brain cells, and maintain cardiac action, respectively. The brain is unique relative to systemic circulation in that its cells are passively perfused throughout systole and diastole by pulsatile flow while its smallest arteries are exposed to high pulsatile pressure. ${ }^{50}$ Understanding the role of these smaller arteries in head injury may provide some insight into the mechanism for reductions in cerebral blood flow and, through measurement tools like the Brain Acoustic Monitor, provide an objective means of diagnosis and identification of recovery.

The arterial pressure waveform, which is what is provided by the BAM signals, allows for calculation of various outcomes that provide insight into the presence of arterial stiffening and alterations in arterial function. A normal arterial waveform starts with a steep systolic upstroke during the systolic ejection that then declines as left ventricular ejection decreases. ${ }^{48,75}$ It is then followed, when measured peripherally, by a smooth secondary peak which corresponds from reflected pressure waves from the periphery of the arterial vascular bed. It continues to decline, reaching its lowest point at end diastole. Pressure waveforms from different sites of the arterial tree have different morphologies based on the physical properties of the vascular bed. Moving distally, an arterial pressure waveform will have a higher and steeper systolic peak, a later dicrotic notch, a diastolic wave that is more prominent, and a lower end diastolic pressure than that seen centrally. ${ }^{48}$ 
Several factors, beyond vascular bed properties, affect the shape of the arterial pressure waveform and many have been identified in relation to various pathologies. Age, in particular, results in reduced arterial distensibility naturally and causes earlier pressure wave reflections that eventually augment the systolic pressure peak rather than the diastolic pressure wave. ${ }^{75-77}$ Additionally, characteristic arterial pressure waveforms have been identified in association with congestive heart failure, aortic insufficiency, aortic stenosis, cardiac tamponade, and hypertrophic cardiomyopathy. ${ }^{48}$ Regarding sport-related concussion, it is possible that the uncoupling of the autonomic control of cerebrovascular reactivity can result in similar changes in an arterial pressure waveform, indicating increased pressure within the small vessels of the brain. While this is idea is merely theoretical, the proposed study seeks to describe the cerebrovascular response to concussion using metrics of an arterial pressure waveform obtained acoustically through the BAM.

Specific outcomes used to describe arterial function rely on systolic and wave reflection spacing and height. The measurement of pulse wave velocity is generally accepted as the most simple, non-invasive, robust, and reproducible method to determine arterial stiffness. Carotid-femoral pulse wave velocity is considered the "gold standard" measurement of arterial stiffness. ${ }^{75}$ It is measured using the foot to foot velocity from waveforms measured at the carotid artery and the right femoral artery. The distance between the two recording sites is divided by the time delay, or transit time, to calculate pulse wave velocity. In relation to the BAM, this will be calculated using peak spacing. That is, comparisons will be made using the time delay between the systolic peak and the 
reflected wave peak, with a higher pulse wave velocity indicated with decreases in this time interval. A second outcome commonly used in assessment of arterial stiffness is called augmentation index and it refers to the augmentation of the systolic pressure by an earlier reflection. ${ }^{75}$ It is quantified as the difference between the magnitude of the first and second peaks and is expressed as a percentage of the pulse pressure (systolic pressure). For purposes of our evaluation with the BAM, a smaller augmentation index would be indicative of increased arterial stiffness.

In addition to its clinical application as a predictor of cardiovascular disease, measures of arterial stiffness have also been implicated in cognitive decline in the absence of dementia. Suggested mechanisms include augmented pressure pulses, resulting from arterial stiffness, that penetrate and damage small cerebral vessels, particularly in areas that control cognitive functions. A study by Pase et al. in 2010 examine a sample of middle-aged individuals with no history of cardiovascular disease, diabetes, stroke, hypertenision, or use of medications to determine how measures of arterial stiffness affected specific domains of cognitive performance. ${ }^{77}$ They found that pulse pressure was an independent predictor of both episodic and secondary memory performance and speed of memory retrieval while augmentation index was an independent predictor of speed of memory. Pulse pressure has been similarly shown to predict secondary memory elsewhere. ${ }^{78}$ These findings suggest that healthy, nonclinical populations may experience decrements in cognitive function in the presence of increased arterial stiffness. Because similar deficits in cognition are seen in concussed individuals, it may be of interest to determine whether arterial stiffening leads to the previously 
mentioned decreases in blood supply. It is also possible that the mechanical forces experienced during a lifetime of athletic activity, especially in the case of chronic exposure to collision sports, can lead to similar damage in small cerebral vessels as seen with aging as a result from augmented pressure.

\section{Current Concussion Assessment}

Current standards of care for concussed athletes involve a multi-faceted approach. The National Athletic Trainers' Association position statement on concussion recommends standardized methods of measuring post-concussion signs and symptoms, cognitive dysfunction, and postural instability. Over the last 5 years, the use of brief screening tools to assess athletes on the sideline immediately post-injury and later neurocognitive testing to track recovery have become commonplace. Baseline testing for all forms of assessment provides an indicator of what is "normal" for a particular athlete while also establishing the most accurate and reliable marker of recovery.

\section{Sideline assessment}

Most sideline assessment tools upon original development assessed single domains of concussion effects such as post-concussion symptoms, neurocognitive function, or postural control. In 2004, the International Committee on Concussion in

Sport developed the Sideline Concussion Assessment Tool (SCAT) ${ }^{79}$ This was replaced by a second version (the SCAT2) in 2008 and a third version was released in March 2013. ${ }^{4,80}$ The SCAT3, the most recent version of the SCAT battery, includes symptom ratings, balance testing, and cognitive screening. 
A number of symptoms may result from the underlying neurometabolic cascade that occurs following concussive injury. Typical symptoms reported following concussion include but are not limited to headache, dizziness, nausea, vomiting, feeling "in a fog", feeling "slowed down", having trouble falling asleep, sleeping more or less than usual, fatigue, drowsiness, sensitivity to light or noise, unsteadiness or loss of balance, feeling "dinged", dazed, or stunned, seeing stars or flashing lights, ringing in the hears, and double vision. Many post-concussion symptom inventories have been developed with the goal of allowing repeated administration to track recovery and resolution of symptoms. Regardless of the form used, obtaining a baseline symptom inventory is important to establish any pre-existing symptoms that may be due to other factors beyond head injury, such as illness, fatigue, psychological disorder, or migraine. These and other factors such as age and sex have shown to produce differences in baseline and post-concussion symptomology and recovery of symptoms.

The most commonly reported symptoms in a study by Gessel et al. among high school athletes were headache $(40.1 \%)$ followed by dizziness $(15.3 \%)$, and confusion $(8.6 \%) .{ }^{21}$ Other symptoms included loss of consciousness $(3.9 \%)$ and amnesia $(6.4 \%)$. Similarly, in 2011, Castille and colleagues found the most commonly reported symptom for all concussions was headache $(85.5 \%)$ followed by dizziness $(64.6 \%)$, concentration difficulty (47.8\%), and confusion/disorientation (39.5\%). ${ }^{24}$ A mean of 3.61 symptoms were reported per concussion. Compared to symptoms produced from recurrent concussions, they found that light and noise sensitivity (37.0\% vs. $29.6 \%)$ and loss of consciousness $(7.7 \%$ vs. $4.4 \%)$ were more frequent with recurrent than with new 
concussions. Comparing sexes, boys reported amnesia more often than girls $(31.1 \%$ vs. $13.1 \%$ ) for new concussions, but these proportions were more similar in recurrent concussions (16.3\% vs. 13.6\%). Among their sample, the highest proportion of first-time concussion symptoms resolved in 1 day to 1 week. For recurrent concussions, in seven of nine sports, the highest proportion of symptoms resolved in 1 day to 1 week. Number of symptoms was associated with symptom resolution time. For example, among new concussions with greater than 4 symptoms, $20.6 \%$ resolved in greater than 1 week compared with $9 \%$ of new concussions with less than 4 symptoms. Among recurrent concussions with greater than 4 symptoms, $36.6 \%$ resolved in greater than 1 week compared with $19.1 \%$ of recurrent concussions with less than 4 symptoms. ${ }^{24}$ These differences emphasize the need for adequate pre-participation histories such that comorbidities and prior history of concussion can be noted, particularly in the instance of subsequent concussion, so a prolonged recovery or differences in symptomology might be understood and explained during concussion management.

Also included in sideline concussion assessments are brief tests of cognitive function. Specifically, the SCAT3 includes the Standardized Assessment of Concussion, which assesses orientation, immediate and delayed memory, and concentration through questions regarding the date, time, and year, three trials of a 5-word recall and a delayed recall trial, recitation of a string of digits in reverse, and recitation of the calendar months in reverse. This portion of the sideline evaluation, deemed "essential" by the recently released consensus statement on concussion in sport, largely operates to serve as a "rapid screening tool" and is not meant to replace more advanced and comprehensive 
neurocognitive testing nor is it designed to serve as a stand-alone tool for the ongoing management of sport-related concussion. ${ }^{4}$ A large number of studies have been performed to determine the ability of the SAC and other brief cognitive tests to identify cognitive deficits in concussed individuals. Most studies did detect deficits on day-ofinjury assessments; however, there is large variation in recovery to baseline levels among different severities of injury. ${ }^{81-85}$

Many studies have been published over the last five years with the goal of establishing normative values in various populations. ${ }^{86,87}$ Consistent recommendations among these studies are that the high-rate of failure among non-concussed athletes on the tests included in the SCAT battery indicates an inaccurate discriminatory ability without individual baseline tests. For instance, Jinguji in 2012 performed a study to establish normative SCAT2 values for high school athletes. ${ }^{86}$ They found that only $41 \%$ and $17 \%$ of athletes could recite strings of 5 or 6 digits backwards, respectively. Also, only $67 \%$ of their sample could repeat the months of the year backwards. These findings suggest that reliance on normative data would result in a high rate of false positive and false negative scores. A similar study by McCleod in 2012 found that male athletes, $9^{\text {th }}$ graders, and those with prior history of concussion scored lower on the SCAT2 than females, upper classmen, and those with no history of concussion. They, too, recommended that clinicians administer baseline assessments of the SCAT battery because reliance upon a perfect score of 100 is not appropriate in the high school population.

A final component of the sideline assessment of concussion is an evaluation of balance. Disruption of balance, both statically and dynamically, has been identified and 
described in a number of pathologic conditions. Following concussion, balance problems have been should to persist in $30 \%$ of individuals. ${ }^{22}$ Using high-technology and lowtechnology methods of balance assessment, balance deficits have been identified and shown to recover within 3 to 10 days post-injury. ${ }^{88}$ Using a test called the modified CTSIB, concussed subjects demonstrated decreased postural stability compared to their own baseline scores and to their matched controls during the initial 3 days post-injury. Other studies using an instrumented balance assessment called the Sensory Organization Test have identified sensory interaction and balance deficits that typically resolve within 3 to 5 days post-injury. ${ }^{88-93}$ Two possible mechanisms for balance dysfunction following concussion include damage to peripheral receptors that provide inaccurate senses of motion or impairment of the brain centers responsible for central integration of vestibular, visual, and somatosensory information. ${ }^{94}$

Included in the SCAT2 and SCAT3 testing batteries is a more clinically applicable and cost-effective method of assessing balance in athletes - the Balance Error Scoring System or BESS. This test is often performed on the sideline and, in its modified version, only requires the use of a stop-watch. Athletes are asked to assume three stances on firm and foam surfaces while placing their hands on the hips and closing their eyes for 20 seconds. During the stances, the clinician sums 1 point for each error committed. This test has shown to identify balance deficits in concussed individuals 3 to 7 days post injury. ${ }^{95}$ McCrea and colleagues reported that BESS scores in concussed collegiate athletes were 5.7 points below their own baseline on the day of injury, and only 2.7 points below baseline 1 day post-injury. ${ }^{95}$ Another study found impairment in $36 \%$ of 
injured subjects immediately following concussion with a remaining $24 \%$ impaired at 2 days post-injury, and $9 \%$ impaired at 7 days post-injury. ${ }^{96}$ The authors also calculated sensitivity and specificity for the BESS at each of those time points, with the highest sensitivity at the time of injury (0.34). Specificity ranged from 0.91 to 0.96 across days 1 to 7.

The BESS has shown to significantly correlate with force platform sway measures and the Sensory Organization Test with correlations coefficients ranging from 0.78 to $0.96{ }^{97}$ Clinicians need be aware that performance on the BESS can be influenced by a number of factors including the type of sport played, history of ankle injury and instability, and exertion and fatigue. ${ }^{98-101}$ Also, a learning effect may need to be accounted for. ${ }^{102,103}$ It should be noted that while the BESS is able to distinguish concussed individuals without the use of brief cognitive screening tools, its sensitivity is improved when used in combination with them.

\section{Computerized Neurocognitive Assessments}

Computerized neuropsychological (or neurocognitive) assessment using comparison of pre-injury and post-injury performance to determine recovery of neurocognitive function is likely the most objective and widely-used form of concussion assessment currently. As such, many tests are now commercially available, though no recommendations exist regarding superiority of one test over another. Some of these tests used over the past decade include the Automated Neuro-Psychological Assessment Metrics (ANAM), the Immediate Post-Concussion Assessment and Cognitive Testing batter (ImPACT), Concussion Resolution Index, CogSport, and, more recently, 
Concussion Vital Signs (CVS). For purposes of this review, ImPACT and CRI will be described.

The Concussion Resolution Index is a web-based computerized neurocognitive testing battery developed for comparison of an athlete's post-concussion performance with his or her baseline performance. It consists of measures of cognitive function such as memory, reaction time, speed of decision making, and speed of information processing speed. Six subtests are administered at baseline and again at post-trauma evaluation. The overall performance on each post-trauma CRI administration is depicted by a "traffic light" system and classified as red, yellow, or green. This classification system was developed using statistical tests that accounted for a "reliable change" from baseline and practice effects. Red lights reflect neurocognitive function that is significantly different from baseline or 3 or more symptoms self-reported. Yellow lights indicate borderline neurocognitive test results or presence of 1 or 2 post-concussive symptoms on symptom inventory. Green lights indicate non-significant differences in any measures compared to baseline and no self-reported post-concussion symptoms. A report containing the "traffic light" rating and recommendations regarding clinical care is automatically generated at the conclusion of each post-trauma administration.

The subtests used during the CRI include animal decoding, symbol scanning, reaction time, cued reaction time, and visual recognition 1 and 2. During the animal decoding test, athletes are instructed to type in numbers keyed to pictures of animals. For symbol scanning, athletes are instructed to rapidly determine if an identified set of symbols are present among a larger set of symbols. These two subjects are combined to 
form a processing speed index. For reaction time, athletes are instructed to press a space bar when a target shape appears on the screen. This is combined with cued reaction time, during which athletes press the spacebar when a target shape appears immediately after a "cue" shape, to form a simple reaction time index. An error index is also calculated with these measures using total false-positives and false-negatives. Lastly, visual recognition 1 and 2 involve a series of pictures, some of which are repeated. Athletes are instructed to press the spacebar when they recognize a picture from an earlier exposure. The time to response or response latency is recorded during this task and combined with a second similar but delayed task to form a complex reaction time index. As with simple reaction time, an error score is calculated based on total false-positive and false-negative responses.

The five CRI indices and subtests have been shown to be correlated with traditional neurocognitive and neuropsychological measures. ${ }^{104}$ For instance, the processing speed index showed correlation coefficients of $0.66,0.60,0.57$, and 0.58 with the Symbol Digit Modality Test, the Grooved Pegboard Test (dominant and nondominant hands), and the WAIS-III Symbol Search subtest, respectively. Similarly, the simple reaction time measure has shown correlation coefficients of $0.46,0.60$, and 0.56 with the Grooved Pegboard Test (dominant and non-dominant hands) and Trail-Making Test Part A, respectively. For the complex reaction speed index, correlations with the dominant and nondominant hand Grooved Pegboard tests were 0.59 and 0.70,. These correlations, while moderate, indicate that the CRI is able to measure similar constructs in normative subjects. 
One area of concern for neurocognitive tests such as the CRI are in the influence of repeated testing and learning effects on the reliability of scores over time. Two-week test-retest reliabilities for the CRI indices have been shown to be 0.90 for processing speed, 0.73 for simple reaction time, and 0.72 for complex reaction time in collegiate and adult club athletes. ${ }^{104}$ In high school athletes, two week test-retest reliabilities were 0.79 for processing speed, 0.72 for simple reaction time, and 0.65 for complex reaction time. These reliabilities compare well with those previously mentioned regarding the validity of the CRI.

The CRI has been shown to be relatively sensitive to concussion, largely because of its inclusion of self-reported symptoms in its "traffic light" system. In a study by Erlanger and colleagues in 2001, $88 \%$ of 26 concussed athletes were flagged upon initial evaluation using the CRI. ${ }^{104}$ Three of those flagged had positive findings due to decreased cognitive performance in comparison with their own baseline performances in the absence of neurophysiologic symptoms. In those (14 athletes) who underwent a second evaluation, 12 showed evidence of ongoing cognitive or neurophysiologic symptoms, or both, with 7 classified as red and 5 as yellow. One quarter of these athletes were identified as symptomatic based on their cognitive measurements alone. These researchers found that the most sensitive of the CRI indices was complex reaction time. This outcome was significantly different (worse) compared to baseline in $50 \%$ of the sample, with a mean effect size of -1.44 . The second most-sensitive was simple reaction ime, with borderline (yellow) to significant (red) results in $42 \%$ of the sample and an 
effect size of -1.39 . Processing speed index showed significant results in $15 \%$ of the sample with an effect size of $-0.06 .^{104}$

The ImPACT battery consists of seven individual cognitive test modules that form composites in verbal memory, visual memory, reaction time, and processing speed (or visual-motor speed). The verbal memory composite represents the average percent correct for a word recognition task, a symbol-digit match task, and a letter memory task with an accompanying interference task. The visual memory composite is represented by the average percent correct scores for an abstract figure recognition task and memory of a series of illuminated X's and O's after an intervening sequential number clicking task. The reaction time composite represents the average response time on a choice reaction time task, a go or no-go task, and the symbol-number match task. The processing speed or visual motor speed composite consists of the weighted average of three interference tasks during the visual and verbal memory tests. Another outcome given by the ImPACT battery that has been deemed useful for the identification of athletes who are not putting forth maximum effort or who are seriously confused about test instructions is the impulse control composite. This composite is calculated by summing the number of errors of omission or commission on the go/no-go task and the choice reaction time test. The ImPACT test, similar to the CRI, also includes a post-concussion symptom scale that asks athletes to rate the presence and severity of symptoms commonly associated with concussion.

Validity of the ImPACT has been examined through correlations with preexisiting neuropsychological tests. ${ }^{105-107}$ Performance on the Symbol-Digit Modalities 
test has been shown to correlate significantly with the processing speed composite $(r=$ $0.70)$ and reaction time $(\mathrm{r}=-0.60) .{ }^{105}$ Moderate correlations have also been shown between the Brief Visuospatial Memory Test (BVMT) total score and the two ImPACT memory composites as well as the delayed recall score of the BVMT and all five ImPACT outcomes. Additionally, the processing speed composite was shown to be moderately correlated with the Trail-Making Test Parts A and B. ${ }^{105}$ These values are similar to those obtained using processing speed and reaction time outcomes of the CRI. Test-retest reliability of the ImPACT test has been shown using Pearson test-retest correlation coefficients and intra-class correlation analysis. ${ }^{106}$ A study of 56 adolescents and college-aged students showed pearson's correlation coefficients to range from 0.65 (Post-concussion scale) to 0.86 (Processing speed) when tested an average of 5.8 days apart. ${ }^{106}$ Intraclass correlation coefficients were calculated using a two-year testing interval in collegiate athletes and a range of 0.43 (symptom scale) to 0.74 (processing speed) was found. These findings have aided in clinical recommendations for annual baseline testing in high school athletes and bi-annual testing in collegiate athletes. ImPACT has also shown to successfully identify concussed individuals. In a sample of 41 concussed adolescent and collegiate athletes, a significant decline in verbal memory (effect size, 0.82), visual memory (effect size, 0.69), processing speed (effect size, 0.49 ), and reaction time (effect size, 0.95 ) were found 72 hours post-injury using dependent $\mathrm{t}$ tests. ${ }^{106}$ Using a reliable change score that takes into account practice effects, the percentages of athletes to show decline were changed. No decline in any of the four composites or symptom report was identified in $24.4 \%$, one decline in $12.2 \%$, two 
declines in $14.6 \%$, three declines in $17.1 \%$, four declines in $19.5 \%$, and five declines in $12.2 \%$. A total of $44-54 \%$ of the 41 concussed athletes used in this study showed decline in at least 1 domain of the ImPACT test.

A more recent study by Schatz and colleagues found better discriminant ability of the ImPACT to identify concussed individuals. ${ }^{108}$ In this study of high school athletes, $82 \%$ of concussed athletes and $89.4 \%$ of control athletes were accurately categorized. A positive likelihood ratio was determined to be 7.73 and the negative likelihood ratio was 0.20 . More recently, the same authors examined the sensitivity of the ImpACT in samples of symptomatic concussed high school and collegiate athletes and asymptomatic concussed high school and collegiate athletes suspected of hiding post-concussion symptoms. ${ }^{109}$ A group of 81 symptomatic concussed athletes and 37 asymptomatic athletes suspected to sustain a concussion were observed and all subjects took the ImPACT within 3 days of their injury. For those who were symptomatic, sensitivity and specificity was found to be $91.4 \%$ and $69.1 \%$, respectively. For those who were asymptomatic, ImPACT yielded $94.6 \%$ sensitivity and $97.3 \%$ specificity. These more recent findings suggest that the ImPACT is a valid measure of neurocognitive performance in the acute stage of concussion and is sensitive and specific, even when athletes appear to be denying post-concussion symptoms. This is also greater than that established for paper-based measures such as the Hopkins Verbal Learning Test, Trail Making Test, the Symbol Digit Modalities Test and the Controlled Oral Word Association Test. ${ }^{110}$ At 24 hours post-injury, the Digit-Span Test was least sensitive to concussion with successful identification of abnormal function in $30.4 \%$ of athletes. 
COWAT, Hopkins Verbal Learning Test, Trail Making Tests A and B, and Symbol Digit Modalities test showed 34.8\%, 39.1\%, 52.2\%, and 52.2\% sensitivity, respectively. This was poor in comparison to findings in a similar population using ImPACT and CRI which showed $79.2 \%$ and $78.6 \%$ sensitivity, respectively.

Examination of sub-groups of concussed athletes based on severity of initial onfield symptoms has shown that individuals with more severe symptoms, identified by presence of retrograde amnesia, anterograde amnesia, or disorientation for greater than 5 minutes, demonstrate larger decreases in ImPACT composites from baseline and take longer to return to baseline than concussed athletes in a "less severe" group identified by no mental status changes or mental status changes that lasted less than 5 minutes. ${ }^{111}$ Both studies described here indicate that the ImPACT battery is a sensitive and specific instrument for assessment of the neurocognitive and neurobehavioral sequelae of concussion in both severe and milder cases. 


\section{APPENDIX C}

Additional Methods

Table C1: University of Virginia Institutional Review Board Approved Protocol (\#16857)

\section{IRB-HSR PROTOCOL}

\section{Investigator Agreement}

\section{BY SIGNING THIS DOCUMENT, THE INVESTIGATOR CONFIRMS:}

1. I am not currently debarred by the US FDA from involvement in clinical research studies.

2. I am not involved in any regulatory or misconduct litigation or investigation by the FDA.

3. That if this study involves any funding or resources from an outside source, or if you will be sharing data outside of UVA prior to publication that you will contact the Dean's office regarding the need for a contract and letter of indemnification. If it is determined that either a contract or letter of indemnification is needed, subjects cannot be enrolled until these documents are complete.

4. The proposed research project will be conducted by me or under my close supervision. It will be conducted in accordance with the protocol submitted to and approved by the IRB including any modifications, amendments or addendums submitted and approved by the IRB throughout the life of the protocol.

5. That no personnel will be allowed to work on this protocol until they have completed the IRB-HSR On-line training and the IRB-HSR has been notified.

6. That all personnel working on this protocol will follow all IRB-HSR Policies and Procedures as stated on the IRB-HSR Website http://www.virginia.edu/vprgs/irb/ and on the School of Medicine Clinical Trials Office Website: http://knowledgelink.healthsystem.virginia.edu/intranet/hes/cto/sops/sop_index.cfm

7. I will ensure that all those delegated tasks relating to this study, whether explicitly or implicitly, are capable through expertise, training, experience or credentialing to undertake those tasks.

8. I confirm that the implications of the study have been discussed with all Departments that might be affected by it and have obtained their agreement for the study to take place.

9. That no subjects will be recruited or entered under the protocol until the Investigator has received the signed IRB-HSR Approval form stating the protocol is open to enrollment

10. That any materials used to recruit subjects will be approved by the IRB-HSR prior to use. 
11. That all subjects will sign a copy of the most current consent form that has a nonexpired IRB-HSR approval stamp.

12. That any modifications of the protocol or consent form will not be initiated without prior written approval from the IRB-HSR, except when necessary to eliminate immediate hazards to the subjects.

13. Any significant findings that become known in the course of the research that might affect the willingness of subjects to enroll or to continue to take part, will be promptly reported to the IRB.

14. I will report immediately to the IRB any unanticipated problems involving risk to subjects or to others including adverse reactions to biologics, drugs or medical devices.

15. That any serious deviation from the protocol will be reported promptly to the Board in writing.

16. That any data breach will be reported to the IRB, the UVa Corporate Compliance and Privacy Office, UVa Police as applicable.

17. That the continuation status report for this protocol will be completed and returned within the time limit stated on the form.

18. That the IRB-HSR office will be notified within 30 days of a change in the Principal Investigator or of the closure of this study.

19. That a new PI will be assigned if the current PI will not be at UVA for an extended period of time. If the current PI is leaving UVa permanently, a new PI will be assigned PRIOR to the departure of the current PI.

20. All study team members will have access to the current protocol and other applicable documents such as the IRB-HSR Application, consent forms and Investigator Brochures.

21. Signed consent forms and other research records will be retained in a confidential manner. Records will be kept at least 6 years after completion of the study.

22. No data/specimens may be taken from UVa without a signed Material Transfer Agreement between OSP/SOM Grants and Contracts Office and the new institution. Original study files are considered institutional records and may not be transferred to another institution. I will notify my department administration regarding where the originals will be kept at UVa. The material transfer agreement will delineate what copies of data, health information and/or specimens may be taken outside of UVa. It will also approve which HIPAA identifiers may be taken outside of UVa with the health information or specimens.

23. If any member of study team leaves UVa, they are STRONGLY ENCOURAGED to use Exit Checklist found on IRB-HSR website at http://www.virginia.edu/provost/facultyexit.pdf.

The IRB reserves the right to terminate this study at any time if, in its opinion, (1) the risks of further experimentation are prohibitive, or (2) the above agreement is breached.

\section{Investigators Experience}


The investigators included on this protocol have extensive research experience working with an athletic population both at the high school and collegiate levels.

Dr. Broshek is -Director of the Neurocognitive Assessment Laboratory at UVa and an associate director of the Brain Injury and Sports Concussion Institute. She also provides psychotherapy to student athletes as well as consultation regarding concussion management. She has worked in the area of sports concussion for 12 years.

T. Jason Druzgal, MD, PhD is an Assistant Professor of Radiology at the University of Virginia. He runs the University of Virginia Functional Neuroradiology Laboratory. He is board certified in clinical neuroradiology and has been performing magnetic resonance imaging research on the human brain since 1998. He has specific ex.pertise in using functional MRI in the evaluation of normal cognition and cognitive disorders. His prior work has included patient populations with autism, movement disorders, and traumatic brain injury

Dr. Saliba is the Primary Investigator and an Assistant Professor within and director of the Kinesiology program within the Curry School of Education. She has performed extensive research using this population and has worked clinically as an athletic trainer for more than 10 years.

Shellie Spiers is a Sports Medicine doctoral candidate within the Kinesiology program at the University of Virginia. She has been performing research and working clinically as an athletic trainer with the active population for the past 5 years.

Bryson Reynolds is a graduate student in the neuroscience graduate program at the University of Virginia. The proposed study represents a central component of his dissertation project. Bryson has undergraduate training in psychology and has 2 years experience with collection and analysis of neuroimaging data.

Jasmine Manalel is a research assistant to Jason Druzgal. She has a B.S. Psychology from the University of Virginia and 9 months experience with the collection and analysis of neuroimaging data.

Joella Matheny,Shari Benson, Thomas Lilley, and Shannon Snell are Certified Athletic Trainers at three local Charlottesville high schools. They each have 5 or more years of experience working clinically with the high school population.

Sam Walton is a certified athletic trainer working at Longwood University. He has been working with an athletic population for the past 5 years and is well-versed in concussion recognition and management, including the tools used for assessment that will be used in this study. He has also served as a research assistant on other UVA-initiated concussion research studies. 


\section{Signatures}

\section{Principal Investigator}

Principal Investigator

Signature
Principal Investigator $\quad$ Date

Name Printed

\section{Department Chair}

BY SIGNING THIS DOCUMENT THE DEPARTMENT CHAIR AGREES:

1. To work with the investigator and with the board as needed, to maintain compliance with this agreement.

2. That the Principal Investigator is qualified to perform this study.

3. That the protocol is scientifically relevant and sound.

Department Chair or Designee

Signature $\overline{\text { Department Chair or Designee }}$

Name Printed
Date

The person signing as the Department Chair cannot be the Principal Investigator or a sub-investigator on this protocol.

The Department Chair or Designee signature is ONLY required if this is a new protocol or a modification changing the Principal Investigator

\section{Brief Summary/Abstract}

The purpose of this study will be to determine if there are detectable changes in cerebral blood flow, as measured by an investigational device called the BAM, immediately after concussion (or mild traumatic brain injury). This device measures the acoustic signals emitted by the cerebral arteries and creates an arterial pressure wave. With this wave, we are able to calculate various metrics of arterial stiffness and our goal is to determine if changes in these measures are detectable immediately after concussion. We also aim to see how long it takes for blood flow measures to return to baseline following a concussive head injury. Our hypothesis would be that there are alterations in cerebral blood flow measures immediately following concussion and that these changes will revert to baseline values after a 7 to 10 day period. To determine if this is true, we will perform baseline measures with the BAM on student athletes at the University of Virginia and two local high schools prior to the initiation of sport participation at the 
beginning of a competitive season and repeat these measures serially if an individual sustains a concussion. We plan to assess athletes from the football teams, men's and women's lacrosse, men's and women's soccer, and men's and women's basketball.

\section{Background}

Changes in cerebral blood flow velocity and vasospasm of the cerebral vessels are a common occurrence following traumatic brain injury. It is unknown if these changes are seen in milder cases of head injury such as sports-related concussion. Several parameters have been developed to quantify arteriole stiffness using arterial pressure waveforms of central and peripheral arteries. These stiffness measures have been used to identify individuals at risk of heart disease and also have been associated with the cognitive decline seen with increasing age. The Brain Acoustic Monitor, or BAM, is an investigational device that will measure an acoustic signal given by the cerebral arteries and a reference (most often a digital artery or the radial artery) and displays the pressure pulse wave similarly to that achieved using tonometry. The BAM has been used in cases of severe head trauma to predict outcomes and has been strongly associated with CT scan findings following severe TBI. It has not, however, been used in mild traumatic brain injury patients such as athletes who sustain sports concussion. If it proves useful in detecting changes in arterial stiffness following concussion, the sports medicine profession will gain a technique for measuring a truly physiological change seen in concussion patients that cannot be altered by patient effort or deceitfulness (i.e. hiding symptoms) as most concussion assessment tools are today. Therefore, the goal of this study would be to determine if changes in cerebral blood flow are seen acutely (i.e. on the day of injury) and, if so, how they change during the recovery process. We will also seek to determine if changes in BAM measures correlate with changes in current methods of concussion assessment (neuropsychological testing).

Photographs of the computer and sensors that make up the device are included below.

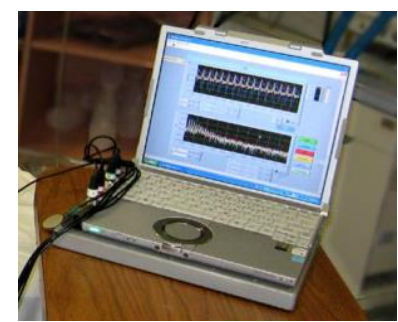

Brain Acoustic Monitor computer and analog to digital converter with data collection software displayed on the computer monitor.
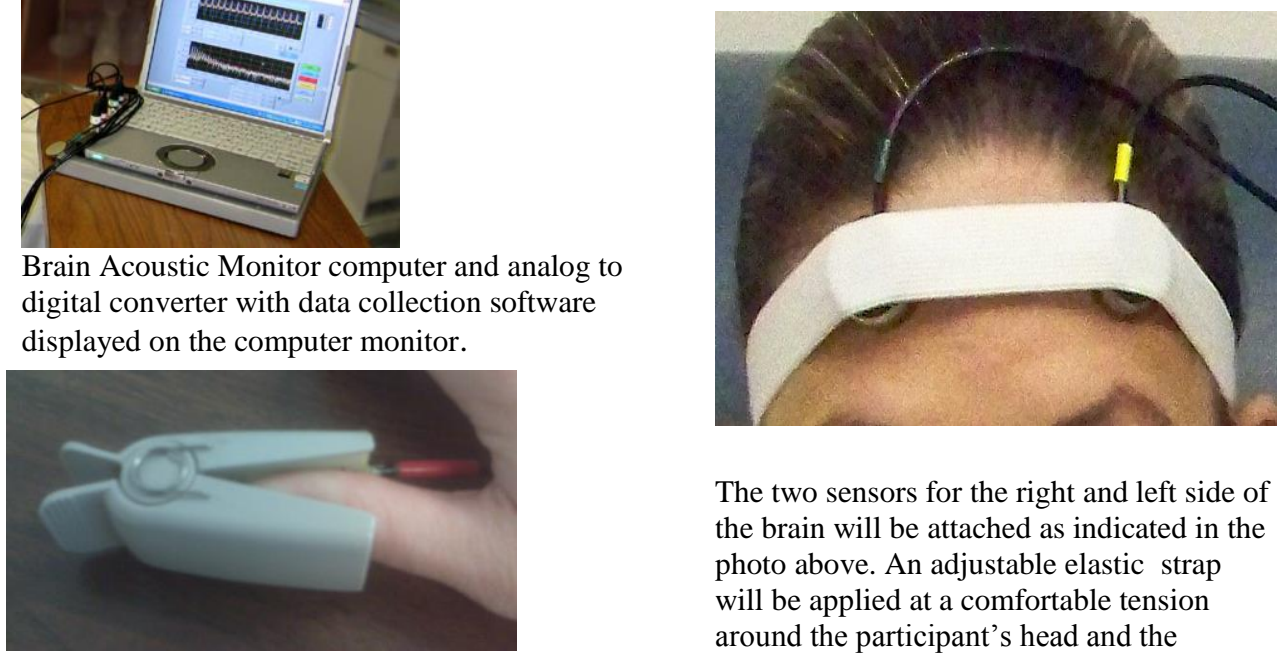

The two sensors for the right and left side of the brain will be attached as indicated in the photo above. An adjustable elastic strap will be applied at a comfortable tension around the participant's head and the sensors will be placed between the elastic strap and the forehead. No energy is eleased

Thumb sensor used to collect the reference acoustical pulse wave to which right and left brain pulse waves will be compared. by these sensors. They only record coustical signals that are given off by the cerebral arteries. 


\section{Hypothesis to be Tested}

Objective \#1: To determine if there are age- and gender-related differences in BAM measures in male and female high school and collegiate athletes.

Objective \#2: To determine if there are acute changes in BAM measures following concussion.

Objective \#3: To determine if changes in BAM measures following concussion correlate with changes in neuropsychological testing.

Objective \#4: To determine if changes in BAM measures are still existent at time of return to activity.

Hypothesis \#1: Adolescents (13-17) and college-aged (18-24) athletes will not differ in blood flow values obtained by the BAM and females will not differ from males.

Hypothesis \#2: Concussion will result in an increase, or worsening, of arterial stiffness values compared to baseline.

Hypothesis \#3: Changes in arterial stiffness will correlate to the changes seen in neuropsychological testing during the acute phase of injury (immediate to $48 \mathrm{hrs}$ after injury).

Hypothesis \#4: Changes in arterial stiffness outcomes will no longer be present at the time of return to activity.

\section{Will controls be used?}

\section{Study Design: Biomedical}

No.

2. What is the study design?

Lab-based, non-blinded, repeated measures.

3. Does the study involve a placebo?

No.

\section{Human Participants}

Ages 13-29

Sex males and females

Race all

Subjects- see below

1. Provide target \# of subjects (at all sites) needed to complete protocol. 300

2. Describe expected rate of screen failure/ dropouts/withdrawals from all sites. We expect a drop out/withdrawal rate of 15 percent.

3. How many subjects will be enrolled at all sites? 350 


\section{How many subjects will sign a consent form under this UVa protocol? 350}

\section{Inclusion/Exclusion Criteria}

\section{List the criteria for inclusion}

- Participation in intercollegiate or interscholastic athletics at the University of Virginia, Longwood University, St. Anne's Belfield, The Covenant School, Western Albemarle High School, or Charlottesville High School

- Age 13-29

\section{List the criteria for exclusion}

- Lack of baseline neurocognitive test scores

- A self-reported history of neurological disorders that would affect cognition

- A self-reported history of learning disorder or attention-deficit disorder

- Pregnancy

\section{List any restrictions on use of other drugs or treatments.}

No restrictions.

\section{Statistical Considerations}

\section{Is stratification/randomization involved?}

No.

\section{What are the statistical considerations for the protocol?}

Objective \#1: To determine if there are age- and gender-related differences in BAM measures in male and female high school and collegiate athletes. To assess age differences, we will group subjects by age and use analysis of variance with follow-up pairwise comparisons if indicated.

Objective \#2: To determine if there are acute changes in BAM measures following concussion.

Objective \#4: To determine if changes in BAM measures are still existent at time of return to activity.

Objective \#3: To determine if changes in BAM measures following concussion correlate with changes in neuropsychological testing.

We will assess objectives \#2 \& \#4 with the repeated measures ANOVA, with 5 time points. Pairwise comparisons will be used in the case of significant time effect. To assess correlation, we will use pearson's R unless data are non-normally distributed (in which case we will use spearman's rho). We will also use multivariate linear regression to determine if acute measures obtained by the BAM and neuropsychological testing predict time to return to play. 


\section{Do you have an adequate sample size, or is your sample size larger than necessary?}

The sample size is larger than necessary because we are taking a prospective caseseries approach and want to widen our scope for obtaining concussed individuals. Also, due to the novelty of this research and lack of previous data using this device, a larger sample was selected.

\section{What is your plan for primary variable analysis?}

Primary variables include BAM measures and neurocognitive test scores compared between groups [age (adolescent vs. collegiate), gender (male vs. female), and concussion history (no history vs. history)] at baseline. Within subjects comparisons [time at 5 levels (baseline, day of injury, 24 hrs post-injury, $48 \mathrm{hrs}$ post-injury, and time of return to play)] will occur using BAM measures and neuropsychological test scores before and after concussion. For baseline comparisons among groups, we will use $2 \times 2 \times 2$ analysis of variance. We will use 2 (age group) x 5 (time) repeated measures for within-subject comparisons of baseline measures to post-injury measures. All significant ANOVAs will be followed with pairwise comparisons when indicated.

\section{What is your plan for secondary variable analysis?}

We will use pearson's R correlation to determine if post-injury BAM measures correlate with post-injury neuropsychological. We will use multivariate linear regression to determine if acute phase (day $0,1, \& 2$ ) BAM measures and neuropsychological test scores predict time to return to play.

\section{Have you been working with a statistician in designing this protocol?}

No.

\section{Biomedical Research}

\section{What will be done in this protocol?}

\section{Session 1 (all subjects will participate):}

1. Consent and review of eligibility (using data collection form)

2. Assessment using the Sideline Concussion Assessment Tool - 3 (SCAT3).

This is used in clinical practice as a daily assessment tool following a concussion. (i.e. not solely for research purposes)

3. Brain Acoustic Monitor readings are taken (for research purposes only):

a. Sensors are applied using elastic band.

b. Analysis is run using automated software/laptop.

c. Sensors are adjusted to obtain satisfactory reading.

d. 5-10 ten second readings are recorded for use in data analysis, up to 20 readings may be taken. 
**Note: Subjects from all sites will undergo pre-season testing using computerized neuropsychological tests (Cognitive resolution index or ImPACT test) by school athletic trainer as part of normal clinical care. We will be using this information, but the test will be administered by school administrators/athletic trainers at a different time, but prior to athletic participation.

University of Virginia \& Charlottesville High School- Cognitive Resolution Index (CRI)

St. Anne's Belfield School \& Western Albemarle High School- Immediate PostConcussion Assessment and Cognitive Testing (ImPACT) battery Longwood University - CNS Vital Signs

The Covenant School - Cognitive Resolution Index (CRI)

\section{Session 2 (concussed only):}

Subjects will undergo BAM and SCAT3 testing on the day of injury within 12 hours of sustaining a concussion. These measurements will take approximately 20 minutes.

\section{Session 3 through Session 5/6 (concussed only):}

Subjects will undergo BAM testing and the symptom section of the SCAT3 24 hours post injury, 48 hours post injury, $1^{\text {st }}$ asymptomatic day (may coincide with 24 or 48 hour session) and on the day of full return to play. At resolution of symptoms, which can take anywhere from 24-48 hours to 1-2 weeks, concussed individuals will repeat the computerized neuropsychological test (ImPACT or CRI, depending on the test used by the school) to determine if their cognitive function has returned to baseline. The full SCAT3 will also be repeated at this time. Return to play will be determined by the team athletic trainer or physician according to the school policy.

\section{Session 2 (healthy)/ Session 7 (concussed only)}

Subjects will undergo BAM and SCAT3 testing following the end of the competitive athletic season, following same procedures included in steps $2 \& 3$ described in session 1.

Study flow for those who sustain a concussion:

\begin{tabular}{|c|c|c|c|c|c|c|c|}
\hline & $\begin{array}{l}\text { Preseaso } \\
\mathrm{n}\end{array}$ & $\begin{array}{l}\text { Immediat } \\
\text { e Post- } \\
\text { Injury }\end{array}$ & $\begin{array}{l}24 \\
\text { hrs } \\
\text { Post- } \\
\text { Injur } \\
\mathrm{y}\end{array}$ & $\begin{array}{l}48 \\
\text { hrs } \\
\text { Post- } \\
\text { Injur } \\
\mathrm{y}\end{array}$ & $\begin{array}{l}1^{\text {st }} \text { day } \\
\text { asymptomati } \\
\text { c }\end{array}$ & $\begin{array}{l}\text { Day } \\
\text { of } \\
\text { Retur } \\
\text { n to } \\
\text { Play }\end{array}$ & $\begin{array}{l}\text { Post- } \\
\text { seaso } \\
\mathrm{n}\end{array}$ \\
\hline BAM & $X$ & $\mathrm{X}$ & $\mathrm{X}$ & $\mathrm{X}$ & $\mathrm{X}$ & $\mathrm{X}$ & X \\
\hline Full SCAT3 & $X$ & $\mathrm{X}$ & & & $X$ & & $\mathrm{X}$ \\
\hline
\end{tabular}




\begin{tabular}{|l|l|l|l|l|l|l|l|}
\hline $\begin{array}{l}\text { Symptom } \\
\text { inventory } \\
\text { (via SCAT3) }\end{array}$ & & & $\mathrm{x}$ & $\mathrm{x}$ & & $\mathrm{x}$ & \\
\hline $\begin{array}{l}\text { CRI/ImPAC } \\
\mathrm{T}\end{array}$ & $\mathrm{X}$ & & & & $\mathrm{X}$ & & \\
\hline
\end{tabular}

Study flow for those who do not sustain a concussion:

\begin{tabular}{|l|l|l|l|l|l|l|l|}
\hline & $\begin{array}{l}\text { Preseaso } \\
\mathrm{n}\end{array}$ & $\begin{array}{l}\text { Immediat } \\
\text { e Post- } \\
\text { Injury }\end{array}$ & $\begin{array}{l}24 \\
\text { hrs } \\
\text { Post- } \\
\text { Injur } \\
\mathrm{y}\end{array}$ & $\begin{array}{l}48 \\
\text { hrs } \\
\text { Post- } \\
\text { Injur } \\
\mathrm{y}\end{array}$ & $\begin{array}{l}1^{\text {st }} \text { day } \\
\text { asymptomati } \\
\mathrm{c}\end{array}$ & $\begin{array}{l}\text { Day } \\
\text { of } \\
\text { Retur } \\
\text { n to } \\
\text { Play }\end{array}$ & $\begin{array}{l}\text { Post- } \\
\text { seaso } \\
\mathrm{n}\end{array}$ \\
\hline BAM & $\mathrm{X}$ & & & & & & $\mathrm{X}$ \\
\hline SCAT3 & $\mathrm{X}$ & & & & & & $\mathrm{X}$ \\
\hline $\begin{array}{l}\text { CRI/ImPAC } \\
\mathrm{T}\end{array}$ & $\mathrm{X}$ & & & & & & \\
\hline
\end{tabular}

Detailed descriptions of neuropsychological tests:

Cognitive Resolution Index: used as standard of care by the University of Virginia, The Covenant School, and Charlottesville High School as part of standard of care for concussion management and return to play decisions. Student athletes take baseline tests every year at the beginning of the school year. In the event of a concussion, athletes are then re-tested using this test to determine neuropsychological deficit. This is an internetbased assessment designed to measure reaction time and processing speed. It lasts about 20-25 minutes and is administered on a computer. There are 6 subtests: reaction time, cued reaction time, visual recognition 1 , visual recognition 2 , animal decoding and symbol scanning.

Immediate Post-Concussion Assessment and Cognitive Test (ImPACT): used by St. Anne's Belfield School and Western Albemarle High School as standard of care for concussion management and return to play decisions, in a similar pre-injury/post-injury format. The test utilizes six different test modules, which yield five composite scores verbal memory, visual memory, visual motor speed, reaction time, and impulse control.

CNS Vital Signs: used by Longwood University as standard of care for concussion management and return to play decisions. This test utilizes 7 subtests: visual memory, verbal memory, finger tapping, symbol digit coding, stroop test, shifting attention test, and continuous performance test to assess neurocognitive function. It results in the following 9 outcomes: Neurocognitive index, composite memory, verbal memory, visual 
memory, processing speed, psychomotor speed, reaction time, complex attention, and cognitive flexibility.

Standardized Concussion Assessment Tool-3: (SCAT3) This is a test intended for sideline (immediate) assessment of concussion as part of standard of care at all institutions. Beyond the first 24-48 hours following a concussion, our use of this test will be for research purposes only. This test is composed of a 22-item graded symptom assessment (22 points), 2 objective questions regarding loss of consciousness and balance problems (2 points), the Glasgow coma scale (GCS) (5 points), the Maddocks et al. score for orientation (applied only to sideline assessments and concussion diagnosis only), the SAC for orientation, immediate memory, concentration, and delayed recall (30 points), the modified BESS (30 points), and coordination examination (1 point). The total score is 100 (higher being better) and the Maddocks score is totaled separately from the rest out of 5 , where higher is better.

2. List the procedures, in bullet form, that will be done for research as stipulated in this protocol. (e.g. blood tests, EKG, x-rays, surveys, administration of investigational drug/device, randomization to one of two approved drugs)

Do NOT list those procedures which are being ordered for clinical care.

These procedures are being done for research purposes only. Descriptions of each are provided in the previous section ("what will be done in this protocol"):

- Brain Acoustic Monitor Readings

- SCAT 3 (beyond immediate post-injury assessment)

3. Will any of the procedures listed in item \# 2 have the potential to identify an incidental finding? (e.g. MRI or CXR shows possible tumor, blood tests show possibility of leukemia, surveys which reveal depression/ suicidal tendencies.)

No.

4. Do any of the procedures listed above, under question \# 2, utilize any imaging procedures ( e.g. ultrasound, CT scans/ X-rays etc.)? No

\section{Will you be using viable embryos? No}

6. Will you be using embryonic stem cells? No

\section{Data and Safety Monitoring Plan}

This study has been deemed minimal risk. Because this study poses minimal risk to the subject, adverse events will only be collected or recorded if a causal relationship to 
the study intervention is suspected. If any adverse event is considered serious and unexpected, the event must be reported to the IRB-HSR within 7 days from the time the study team receives knowledge of the event.

\section{Definitions}

\subsection{How will you define adverse events (AE)?}

Do not change this answer

An adverse event will be considered any undesirable sign, symptom or medical condition considered related to the intervention. Medical condition/diseases present before starting the intervention will be considered adverse events only if they worsen after starting the study and that worsening is considered to be related to the study intervention. An adverse event is also any undesirable and unintended effect of research occurring in human subjects as a result of the collection of identifiable private information under the research.

\subsection{How will you define an unanticipated problem?}

Do not change this answer

An unanticipated problem is any issue that involves increased risk(s) to participants or others. This means issues or problems that cause the subject or others to be placed at greater risk than previously identified, even if the subject or others do not incur actual harm. For example if a subject's confidentiality is compromised resulting in serious negative social, legal or economic ramifications, an unanticipated problem would need to be reported. (e.g serious loss of social status, loss of job, interpersonal conflict.)

\subsection{What is the definition of a protocol violation?}

Do not change this answer

A protocol violation is defined as any change, deviation, or departure from the study design or procedures of a research project that is NOT approved by the IRB-HSR prior to its initiation or implementation, OR deviation from standard operating procedures, Good Clinical Practices (GCPs), federal, state or local regulations. Protocol violations may or may not be under the control of the study team or UVa staff. These protocol violations may be major or minor violations.

Additional Information: see the IRB-HSR website at http://www.virginia.edu/vpr/irb/HSR_docs/Forms/Protocol_Violations_\%20E nrollment_Exceptions_Instructions.doc

\subsection{What is the definition of a data breach?}


Do not change this answer

A data breach is defined in the HITECH Act (43 USC 17932) as an unauthorized acquisition, access, or use of protected health information (PHI) that compromises the security or privacy of such information.

$\underline{\text { Additional Information may be found on the IRB-HSR Website: Data Breach }}$

2. What risks are expected due to the intervention in this protocol?

The risks should be consistent with those in the consent form (if applicable), although they should be written in technical terms in the protocol and in lay terminology in the consent form.

List the most serious or most frequent risk first

Delete last two rows if no additional risks added. Add additional rows to the table below if needed.

\begin{tabular}{|l|l|}
\hline $\begin{array}{l}\text { Expected Risks related to study } \\
\text { participation }\end{array}$ & Pick One \\
\hline $\begin{array}{l}\text { There is a small risk that breaches } \\
\text { of privacy and/or confidentiality } \\
\text { might occur. The risk of violation } \\
\text { of subject privacy and } \\
\text { confidentiality is minimal due to } \\
\text { the requirements of the privacy } \\
\text { plan in this protocol. }\end{array}$ & \\
\hline $\begin{array}{l}\text { Mild worsening of symptoms } \\
\text { associated with concussion }\end{array}$ & Occurs rarely \\
possible following completion of \\
neurocognitive test battery. Some \\
symptoms may include such as \\
headache, fatigue, dizziness, or \\
difficulty concentrating.
\end{tabular}

3. When will recording and reporting of unanticipated problems/adverse events begin?

_ $\mathrm{X} \_$_ After subject signs consent

After subject begins study intervention

Other (specify)

4. When will the recording/reporting of unanticipated problems/adverse events end? 
_ $\mathrm{x}$ __ Subject completes participation in the protocol

End of intervention

30 days post intervention

Subject completes intervention and follow up period of protocol

Other: (specify)

\section{What is your plan for safety monitoring?}

Do not change this answer

Safety monitoring and aggregate review of adverse events, unanticipated problems, protocol violations and any data breach will be performed by the PI and IRB-HSR through continuation review at least annually.

\section{What is your plan for reporting a Unanticipated Problem, Protocol Violation or Data Breach?}

\section{Do not change this answer}

\begin{tabular}{|c|c|c|c|}
\hline Type of Event & $\begin{array}{l}\text { To whom will it } \\
\text { be reported: }\end{array}$ & $\begin{array}{l}\text { Time Frame for } \\
\text { Reporting }\end{array}$ & How reported? \\
\hline $\begin{array}{l}\text { Unanticipated Problems that } \\
\text { are not adverse events or } \\
\text { protocol violations } \\
\text { This would include a Data } \\
\text { Breach. }\end{array}$ & IRB-HSR & $\begin{array}{l}\text { Within } 7 \text { calendar } \\
\text { days from the time } \\
\text { the study team } \\
\text { received knowledge } \\
\text { of the event. }\end{array}$ & $\begin{array}{l}\text { Unanticipated Problem report } \\
\text { form. } \\
\frac{\text { http://www.virginia.edu/vp }}{\text { rgs/irb/HSR_docs/Forms } / R} \\
\text { eporting_Requirements- } \\
\underline{\text { Unanticipated_Problems.d }} \\
\underline{\text { oc })}\end{array}$ \\
\hline $\begin{array}{l}\text { Protocol Violations } \\
\text { (The IRB-HSR only requires } \\
\text { that MAJOR violation be } \\
\text { reported, unless otherwise } \\
\text { required by your sponsor, if } \\
\text { applicable.) } \\
\text { Or } \\
\text { Enrollment Exceptions }\end{array}$ & IRB-HSR & $\begin{array}{l}\text { Within } 7 \text { calendar } \\
\text { days from the time } \\
\text { the study team } \\
\text { received knowledge } \\
\text { of the event. }\end{array}$ & $\begin{array}{l}\text { Protocol Violation and } \\
\text { Enrollment Exception Reporting } \\
\text { Form } \\
\text { http://www.virginia.edu/vp } \\
\text { rgs/irb/hsr_forms.html } \\
\text { Go to } 3^{\text {rd bullet from the }} \\
\text { bottom. }\end{array}$ \\
\hline
\end{tabular}




\begin{tabular}{|c|c|c|c|}
\hline $\begin{array}{l}\text { Data Breach of Protected } \\
\text { Health Information }\end{array}$ & $\begin{array}{l}\text { ITC: if breach } \\
\text { involves } \\
\text { electronic data } \\
\text { UVa Police if } \\
\text { breach includes } \\
\text { items that are } \\
\text { stolen }\end{array}$ & $\begin{array}{l}\text { As soon as possible } \\
\text { and no later than } 24 \\
\text { hours from the time } \\
\text { the incident is } \\
\text { identified. } \\
\text { As soon as possible } \\
\text { and no later than } 24 \\
\text { hours from the time } \\
\text { the incident is } \\
\text { identified. } \\
\text { IMMEDIATELY. }\end{array}$ & $\begin{array}{l}\text { UVa Corporate Compliance and } \\
\text { Privacy Office- Phone 924-9741 } \\
\text { ITC: Information Security } \\
\underline{\text { Incident Reporting }} \\
\text { procedure, } \\
\underline{\text { http://www.itc.virginia.edu }} \\
\text { security/reporting.html } \\
\end{array}$ \\
\hline
\end{tabular}

\section{Risk/ Benefit Analysis}

\section{What are the potential benefits for the participant as well as benefits which may accrue to society in general, as a result of this study?}

There are no direct benefits to the subjects who will participate in this research study. However, if the brain acoustic monitor (BAM) is able to distinguish concussed vs. healthy individuals and correlates well with current methods of concussion assessment, it would provide sports medicine professionals with a truly objective means of assessing one form of the physiological changes associated with concussion. This could, at minimum, decrease the number of advanced imaging exposures concussed athletes are currently exposed to and expedite referral to emergency medical professionals.

\section{Analyze the risk-benefit ratio.}

The procedures included in this protocol are not invasive in nature and only expose subjects to minimal risk of transiently worsening the neurological, cognitive, and/or physiological symptoms associated with concussion, no more so than one would experience by watching television or sitting through a class following a concussion. Additionally, the procedures used in this protocol do not deviate from the normal clinical care that one would experience after suffering a concussion outside of the addition of the 
BAM monitoring.By determining the BAM's usefulness in concussion assessment and management, it would provide the first portable tool able to evaluate physiologic changes in blood flow to the brain. This could add a truly objective dimension to concussion assessment that is not modified by patient effort, potentially leading to the development of a more efficient and effective battery of tests for use in concussion management.

\section{Bibliography}

McCrory P, Meeuwisse W, Johnston K, et al. Consensus statement on concussion in sport: the 3rd International Conference on Concussion in Sport held in Zurich, November 2008. J Athl Train. Jul-Aug 2009;44(4):434-448.

Meehan WP, 3rd, Bachur RG. Sport-related concussion. Pediatrics. Jan 2009;123(1):114123.

Cohen JS, Gioia G, Atabaki S, Teach SJ. Sports-related concussions in pediatrics. Curr Opin Pediatr. Jun 2009;21(3):288-293.

McClincy MP, Lovell MR, Pardini J, Collins MW, Spore MK. Recovery from sports concussion in high school and collegiate athletes. Brain Inj. Jan 2006;20(1):3339.

Sosin DM, Sniezek JE, Thurman DJ. Incidence of mild and moderate brain injury in the United States, 1991. Brain Inj. Jan 1996;10(1):47-54.

Eckner JT, Kutcher JS. Concussion symptom scales and sideline assessment tools: a critical literature update. Curr Sports Med Rep. Jan-Feb 2010;9(1):8-15.

Dutton RP SJ, Aarabi B, Scalea TM. Preliminary trial of a noninvasive brain acoustic monitor in trauma patients with severe closed head injury. The Journal of Trauma Injury, Infection, and Critical Care. 2002 2002;53:857-863.

Dutton RP PK, Cohen R, Wade C, Sewell J, Fouche Y, Stein D, Arabi B, Scalea TM. Diagnosing mild traumatic brain injury: where are we now? Journal of Trauma Injury, Infection, and Critical Care. 2011;70(3):554-559.

Dutton RP, Van Der Heijden M, Aarabi B, et al. Screening TBI patients with the brain acoustic monitor: Association with CT scan findings and neurologic status at hospital discharge. Clinical Intensive Care. 2005; 16: 97-105.

Dutton RP. Noninvasive assessment of cerebral perfusion with the brain acoustic monitor. In: Vincent JL, ed. 2004 Yearbook of Intensive Care and Emergency Medicine. Berlin: Springer-Verlag; 2004:576 -586. 
LaMonte MP, Sewell J, Bahouth MN, Sewell C. A noninvasive portable acoustic diagnostic system to differentiate ischemic from hemorrhagic stroke. $J$ Neuroimaging. Jan 2005;15(1):57-63.

\section{APPENDIX: Support Source}

1. Describe what will be provided and by whom.

Active Signal Technologies, Inc., is providing training and the BAM device free of charge for use in this study.

2. Do you confirm that you will obtain a contract/ material transfer agreement with the provider via Grants and Contracts/ OSP office?

Yes.

3. Will the Support Source be obtaining data prior to publication or performing monitoring of the study?

No.

\section{APPENDIX: Non- UVa Personnel}

1. Explain the duties of non-UVA personnel on this protocol.

Charlottesville High School Athletic Trainer Joella Matheney, St. Anne's Belfield School Athletic Trainer Sheri Benson, Longwood University Athletic Trainer Sam Walton, The Covenant School Athletic Trainer Renee Smith, and Western Albemarle High School Athletic Trainers TJ Lilley will aid in the SCAT2 assessments immediately following concussive injury (part of clinical practice/concussion assessment policy) and will coordinate communication between study investigators and student athletes (i.e. recruitment).

2. Explain your plans for training and oversight of these personnel.

The role of these personnel is to perform their normal clinical assessment of concussion as they would if they were not part of this study. As such, they will not need training to do so. Oversight is also not necessary for this reason as they are legally obliged to do so per school policy.

3. How do you plan to access any study records the non-UVA personnel might maintain?

They will provide test scores for us following concussion. We will obtain these scores in person during post-concussion testing sessions. 


\section{Will the non- UVA personnel be exposed to any additional risk while working on this protocol?}

No.

\section{List name of any other institution with which they have an affiliation.}

Charlottesville High School (Joella Matheny) and St. Anne's Belfield School (Shari Benson)

Sam Walton - Longwood University

Thomas Lilley - Western Albemarle High School

Renee Smith - The Covenant School

\section{Will the non- UVa personnel have access to UVa patients or their health information along with any HIPAA identifiers prior to consent?}

No. They will not have access to UVa patients or their health information.

- IF YES, check the HIPAA identifiers below they will have access too:

\begin{tabular}{|l|l|}
\hline & 1. Name \\
\hline & $\begin{array}{l}\text { 2. All geographic subdivisions smaller than a state, including street address, city, } \\
\text { county, precinct, zip code, and their equivalent geocodes, except for the initial three } \\
\text { digits of the zip code if, according to the current publicly available data from the Bureau } \\
\text { of the Census: (1) The geographic unit formed by combining all zip codes with the same } \\
3 \text { initial digits contains more than } 20,000 \text { people and (2) The initial } 3 \text { digits of a zip code } \\
\text { for all such geographic units containing 20,000 is changed to 000. }\end{array}$ \\
\hline $\begin{array}{l}\text { 3. All elements of dates (except year) for dates directly related to an individual, } \\
\text { including birth date, admission date, discharge date, date of death; and all ages over } 89 \\
\text { and all elements of dates (including year) indicative of such age, except that such ages } \\
\text { and elements may be aggregated into a single category of age } 90 \text { or older. } \\
\text { [This means you may record the year but not record the month or day of any date } \\
\text { related to the subject if the subject is under the age of } 89 . \text { In addition if the subject is } \\
\text { over the age of } 89 \text { you may not record their age and you may not record the month, day } \\
\text { or year of any date related to the subject ] }\end{array}$ \\
\hline 4. Telephone numbers \\
\hline 5. Fax numbers \\
\hline 6. Electronic mail addresses \\
7. Social Security number \\
\hline 8. Medical Record number \\
\hline 9. Health plan beneficiary numbers \\
\hline 10. Account numbers \\
\hline 11. Certificate/license numbers \\
12. Vehicle identifiers and serial numbers, including license plate numbers \\
\hline 13. Device identifiers and serial numbers \\
\hline 14. Web Universal Resource Locators (URLs) \\
15. Internet Protocol (IP) address numbers \\
16. Biometric identifiers, including finger and voice prints \\
\hline
\end{tabular}


17. Full face photographic images and any comparable images

18. Any other unique identifying number, characteristic, code that is derived from or related to information about the individual (e.g. initials, last 4 digits of Social Security \#, mother's maiden name, first 3 letters of last name.)

19. Any other information that could be used alone or in combination with other information to identify an individual. (e.g. rare disease, study team or company has access to the health information and a HIPAA identifier or the key to the code. )

7. If any items above are checked, list names of and non- UVa affiliated individuals who will have access. N/A

- If any item other than 2 or 3 is checked, tracking of the disclosure by the study team will be required via EPIC.

- If only item 2 or 3 is noted, a HIPAA Data Use Agreement will be required in a contract/ agreement between the unaffiliated investigator and UVa.

\section{APPENDIX: Legal/Regulatory}

\section{Recruitment}

The following procedures will be followed:

- Finders fees will not be paid to an individual as they are not allowed by UVa Policy

- All recruitment materials will be approved by the IRB-HSR prior to use. They will be submitted to the IRB after the IRB-HSR has assigned an IRB-HSR \# to the protocol.

- Only those individuals listed as personnel on this protocol will recruit and or conduct the consenting process with potential subjects.

\section{Retention Incentives}

Any item used by the sponsor/ study team to provide incentive to a subject to remain in the study, other than compensation identified in the Payment section, will be submitted to the IRB for review prior to use. The IRB-HSR will provide the study team with a Receipt Acknowledgement for their records. Retention incentive items are such things as water bottles, small tote bags, birthday cards etc. Cash and gift cards are not allowed as retention incentives.

\section{Clinical Privileges}

The following procedures will be followed:

- $\quad$ Investigators who are members of the clinical staff at the University of Virginia Medical Center must have the appropriate credentials and been granted 
clinical privileges to perform specific clinical procedures whether those procedures are experimental or standard.

- $\quad$ The IRB cannot grant clinical privileges.

- $\quad$ Performing procedures which are outside the scope of the clinical privileges that have been granted may result in denial of insurance coverage should claims of negligence or malpractice arise.

- $\quad$ Personnel on this protocol will have the appropriate credentials and clinical privileges in place before performing any procedures required by this protocol.

- $\quad$ Contact the Clinical Staff Office- 924-9055 or 924-8778 for further information.

\section{Sharing of Data/Specimens}

Data and specimens collected under an IRB approved protocol are the property of the University of Virginia. You must have "permission" to share data/ specimens outside of UVa other than for a grant application and or publication. This "permission" may come in the form of a contract with the sponsor or a material transfer agreement (MTA) with others. A contract/ MTA is needed to share the data outside of UVa even if the data includes no HIPAA identifiers and no code that could link the data back to a HIPAA identifier.

- No data will be shared outside of UVa, beyond using data for a grant application and or publication, without a signed contract/MTA approved by the SOM Grants and Contracts office/ OSP or written confirmation that one is not needed.

- No specimens will be shared outside of UVa without a signed contract/MTA approved by the SOM Grants and Contracts office/ OSP or written confirmation that one is not needed.

\section{Prisoners}

If the original protocol/ IRB application stated that no prisoners would be enrolled in this study and subsequently a subject becomes a prisoner, the study team must notify the IRB immediately. The study team and IRB will need to determine if the subject will remain in the study. If the subject will remain in the study, the protocol will have to be rereviewed with the input of a prisoner advocate. The prisoner advocate will also have to be involved in the review of future continuations, modifications or any other reporting such as protocol violations or adverse events.

Prisoner-Individuals are prisoners if they are in any kind of penal institution, such as a prison, jail, or juvenile offender facility, and their ability to leave the institution is restricted. Prisoners may be convicted felons, or may be untried persons who are detained pending judicial action, for example, arraignment or trial.

For additional information see the OHRP website at http://www.hhs.gov/ohrp/policy/populations/index.html 


\section{APPENDIX: Device Information: (Device being evaluated)}

1. List name of device being evaluated.

Brain Acoustical Monitor

Active Signal Technologies, Inc.

611Q North Hammonds Ferry Road

Linthicum Heights, MD 21090-1322

2. Describe pertinent animal data that is available regarding the safety of this device.

No toxicity/safety research has been performed on animals using this device.

3. Describe pertinent human data that is available regarding the safety of this device.

This device is of insignificant risk to human subjects. The sensors associated with the device essentially act like a stethoscope and only record acoustical signals. They do not emit any type of electrical or mechanical energy into the participant. It has been used in previous UVA studies with HSR IRB approval: IRB\#'s 15622 \& 15871.

The device has been used in the following studies, but the focus of these studies was not to assess safety/toxicity.

Dutton RP SJ, Aarabi B, Scalea TM. Preliminary trial of a noninvasive brain acoustic monitor in trauma patients with severe closed head injury. The Journal of Trauma Injury, Infection, and Critical Care. 2002 2002;53:857-863.

Dutton RP PK, Cohen R, Wade C, Sewell J, Fouche Y, Stein D, Arabi B, Scalea TM. Diagnosing mild traumatic brain injury: where are we now? Journal of Trauma Injury, Infection, and Critical Care. 2011;70(3):554-559.

Dutton RP, Van Der Heijden M, Aarabi B, et al. Screening TBI patients with the brain acoustic monitor: Association with CT scan findings and neurologic status at hospital discharge. Clinical Intensive Care. 2005; 16: 97-105.

Dutton RP. Noninvasive assessment of cerebral perfusion with the brain acoustic monitor. In: Vincent JL, ed. 2004 Yearbook of Intensive Care and Emergency Medicine. Berlin: Springer-Verlag; 2004:576 -586.

LaMonte MP, Sewell J, Bahouth MN, Sewell C. A noninvasive portable acoustic diagnostic system to differentiate ischemic from hemorrhagic stroke. $J$ Neuroimaging. Jan 2005;15(1):57-63.

\section{Have there been any human deaths associated with this device?}


No human deaths have occurred as a result of this device.

5. In how many humans has this device been used previously?

More than 1000 participants.

6. If this protocol will be used in children describe any previous use of this device with children of a similar age range.

This device has not been used in children to date.

\section{Is this device implanted?}

No.

\section{Is this a post-marketing study?}

No.

\section{Does this device have an IDE\# from the FDA?}

No.

\section{IDE Exemption Criteria}

\begin{tabular}{|c|}
\hline A legally marketed device when used in accordance with its labeling \\
\hline $\begin{array}{l}\text { A diagnostic device if it complies with the labeling requirements in } \\
\$ 809.10 \text { (c) and if the testing: } \\
\text { - is noninvasive; } \\
\text { - does not require an invasive sampling procedure that presents } \\
\text { significant risk; } \\
\text { - does not by design or intention introduce energy into a subject; } \\
\text { and } \\
\text { - is not used as a diagnostic procedure without confirmation by } \\
\text { another medically established diagnostic product or procedure; } \\
\text { Additional guidance for an in vitro diagnostic device studies can be found in } \\
\text { "Regulating In Vitro Diagnostic Device (IVD) Studies." } \\
\text { http://www.fda.gov/cdrh/comp/ivdreg.html }\end{array}$ \\
\hline $\begin{array}{l}\text { Consumer preference testing, testing of a modification, or testing of a } \\
\text { combination of devices if the device(s) are legally marketed device(s) [that } \\
\text { is, the devices have an approved PMA, cleared Premarket Notification } \\
510(\mathrm{k}) \text {, or are exempt from } 510(\mathrm{k}) \text { ] AND if the testing is not for the } \\
\text { purpose of determining safety or effectiveness and does not put subjects at } \\
\text { risk; }\end{array}$ \\
\hline A device intended solely for veterinary use; \\
\hline $\begin{array}{l}\text { A device shipped solely for research with laboratory animals and contains } \\
\text { the labeling "CAUTION - Device for investigational use in laboratory } \\
\text { animals or other tests that do not involve human subjects." }\end{array}$ \\
\hline $\begin{array}{l}\text { A custom device : } \\
\text { According to 21CFR812.2(c) (7) a custom device as defined in } 812.3(\mathrm{~b}) \text { is } \\
\text { exempt unless the device is being used to determine safety or effectiveness for }\end{array}$ \\
\hline
\end{tabular}




\begin{tabular}{|c|c|}
\hline & $\begin{array}{l}\text { commercial distribution. A custom device means a device that: } \\
\text { (1) Necessarily deviates from devices generally available or from an applicable } \\
\text { performance standard or premarket approval requirement in order to comply } \\
\text { with the order of an individual physician or dentist; } \\
\text { (2) Is not generally available to, or generally used by, other physicians or } \\
\text { dentists; } \\
\text { (3) Is not generally available in finished form for purchase or for dispensing } \\
\text { upon prescription; } \\
\text { (4) Is not offered for commercial distribution through labeling or advertising; } \\
\text { and } \\
\text { (5) Is intended for use by an individual patient named in the order of a physician } \\
\text { or dentist, and is to be made in a specific form for that patient, or is intended to } \\
\text { meet the special needs of the physician or dentist in the course of professional } \\
\text { practice. }\end{array}$ \\
\hline $\mathrm{X}$ & $\begin{array}{l}\text { NA- None of the items above apply- device determined to NOT be } \\
\text { exempt from IDE regulations. If applicable will submit any } \\
\text { documentation from the sponsor regarding device risk determination ( eg. } \\
\text { significant risk vs non-significant risk) }\end{array}$ \\
\hline
\end{tabular}

\section{- IF you did not check any item in the preceding table complete the following item.}

According to 21CFR812.3(m) a Significant Risk (SR) device study is one that presents a potential for serious risk to the health, safety, or welfare of a subject and Check all applicable items

is intended as an implant; or

is used in supporting or sustaining human life; or

is for use of substantial importance in diagnosing, curing, mitigating or treating disease, or otherwise prevents impairment of human health; or

otherwise presents a potential for serious risk to the health, safety, or welfare of a subject. If this is the only item checked-answer the following questions:

- Is this a class I, II or III device? If you are not sure-See the FDA Device Advice for information to help you make this determination.

- Is there a similar device on the market and if so what is the safety profile of that device? Possibly. The BAM measure acoustic signals emitted by the cerebral arteries and creates 
an arterial pressure wave to measure arterial stiffness during mild traumatic brain injury.

- What would be the risk to the subject if the device failed? There is no risk.

- Is the device removable? Yes

- If YES, list potential risks to subject from removing the device. None.

Note: If the participant must undergo a procedure as part of the investigational study, e.g., a surgical procedure to implant the device, the IRB-HSR must consider the potential harm that could be caused by the procedure in addition to the potential harm caused by the device.

Additional information regarding devices may be found on the IRB-HSR Website under DEVICES

None of the options above apply. Device qualifies as a nonsignificant risk device. IMPORTANT: If you checked this option the protocol will need to be reviewed by the full board to determine if the device is significant or nonsignificant risk. Please refer to full board submissions deadlines on the IRB-HSR.

\section{APPENDIX: Recruitment}

Recruitment includes identifying, review of records to determine eligibility or any contact to determine a potential subjects interest in the study. 
*The UVa HIPAA covered entity is composed of the UVa VP Office of Research, the Health System, School of Medicine, School of Nursing, the Sheila C. Johnson Center, the Exercise and Sports Injury Laboratory and the Exercise Physiology Laboratory.

\section{How do you plan to identify potential subjects?}

To "identify" a potential subject refers to steps you plan to take to determine which individuals would qualify to participate in your study. This does NOT include steps to actually contact those individuals. If your study involves more than one group of subjects (e.g. controls and cases or subjects and caregivers) note below which groups are being identified by the given method.

Check the methods you plan to utilize:

a. ___ Chart Review/ Clinic Schedule Review/ Database Review from a database established for health care operations (departmental clinical database) or quality improvement.

DHHS: Study team requests Waiver of Consent to identify potential subjects.

HIPAA-Allowed under Preparatory to Research if PHI to be accessed.

\section{IMPORTANT}

Keep in mind that PHI in the medical record may only be accessed by individuals who work under the UVa HIPAA covered entity; which means they meet one of the following criteria:

- a UVa student working in the UVa HIPAA Covered Entity*

- a faculty or staff member in a PAID appointment in the UVA HIPAA Covered Entity*

b. Review of a database that was established to keep data to be used for future research such as the CDR, departmental research database or use of data from a separate current active research protocol.

DHHS: Study team requests Waiver of Consent to identify potential subjects.

HIPAA-Allowed under Preparatory to Research if PHI to be accessed. 
IMPORTANT

Keep in mind that PHI in the medical record may only be accessed by individuals who work under the UVa HIPAA covered entity; which means they who meet one of the following criteria:

- a UVa student working in the UVa HIPAA Covered Entity*

- a faculty or staff member in a PAID appointment in the UVA HIPAA Covered Entity*

NOTE: The information from which you are obtaining potential subjects must also have an IRB protocol approval.

\section{IRB\#}

If obtaining information from the Clinical Data Repository (CDR) insert IRB \# 10797.

c. ___ Patients UVa health care provider supplies the UVa study team with the patients contact information without patients knowledge.

DHHS: Study team requests Waiver of Consent to identify potential subjects.

HIPAA-Allowed under Preparatory to Research if PHI will be shared by the health care provider.

\section{IMPORTANT}

Keep in mind that PHI may only be given to individuals who work under the UVa HIPAA covered entity; which means they meet one of the following criteria:

- a UVa student working in the UVa HIPAA Covered Entity*

- a faculty or staff member in a PAID appointment in the UVA HIPAA Covered Entity*

d. Patient obtains information about the study from their health care provider. The patient contacts the study team if interested in participating.

DHHS: NA

HIPAA: Allowed under Health Care Operations If this choice is checked, check 3d-INDIRECT CONTACT below. 
e. _ X__ Potential subjects will not be directly identified. They will respond to an advertisement such as a flyer, brochure etc.

If this choice is checked, check 3d-INDIRECT CONTACT below.

DHHS \& HIPAA: NA

f. Potential subjects have previously signed a consent to have their name in a registry/database to be contacted for future studies of this type.

IRB\# of registry/ database:

DHHS \& HIPAA: NA

_ Charlottesville High School, Western Albemarle High School, Albemarle High School, The Covenant School, Longwood University and the University of Virginia have agreed to allow us access to their sports teams. We will receive permission from school administration before contacting any potential subjects. Recruitment letters and group presentations by the study investigators will be used (i.e. direct contact) to disperse information about the study to student athletes and/or their parents at multiple meetings.

If item \# a, b or c is checked above and if this protocol involves the use of protected health information you confirm the following to be true:

- The use or disclosure is sought solely to review protected health information as necessary to prepare the research protocol or other similar preparatory purposes.

- No PHI will be removed from the UVa covered entity.

- The PHI that the researcher seeks to use or access is necessary for the research purposes.

\section{How will potential subjects be contacted?}

To "contact" a potential subjects refers to the initial contact you plan to take to reach a potential subject to determine if they would be interested in participating in your study. This may include direct contact by such methods as by letter, phone, email or in-person or indirect contact such as the use of flyers, radio ads etc.

If your study involves more than one group of subjects (e.g. controls and cases or subjects and caregivers) note below which groups are being contacted by the given method. 
Check the methods you plan to utilize:

a.__ $\mathrm{x}$ _ Direct contact of potential subjects by the study team via letter, direct e-mail. Members of study team ARE NOT health care providers of patients. Information will not be collected from psychotherapy notes.

Note: Letter, phone, direct email scripts must be approved by IRB prior to use. See IRB-HSR Website for templates.

DHHS/HIPAA: Study team requests a Waiver of Consent and Waiver of HIPAA Authorization to contact potential subjects.

\section{IMPORTANT:}

Keep in mind that if PHI was collected during the identification phase that contact with potential subjects may only be performed by individuals who work under the UVa HIPAA covered entity; which means they meet one of the following criteria:

- a UVa student working in the UVa HIPAA Covered Entity*

- a faculty or staff member in a PAID appointment in the UVA HIPAA Covered Entity*

b.___Potential subjects will be approached while at UVa Hospital or Health Clinic by a person who is NOT a member of their health care team. Information will not be collected from psychotherapy notes.

DHHS \& HIPAA: Study team requests a Waiver of Consent and $a$ Waiver of HIPAA Authorization to contact potential subjects.

\section{IMPORTANT:}

Keep in mind that contacting individuals in a clinical setting may only be performed by individuals who work under the UVa HIPAA covered entity; which means they meet one of the following criteria: 
- a UVa student working in the UVa HIPAA Covered Entity*

- a faculty or staff member in a PAID appointment in the UVA HIPAA Covered Entity*

You should share the following information with the potential subject:

1. Your name

2. Who you are: physician, nurse etc. at the University of Virginia.

3. Why you want to speak with them

4. Ask if you have their permission to explain the study to them

5. If asked about how you obtained their information use one of the following as an option for response.

- DO NOT USE THIS RESPONSE UNLESS YOU HAVE OBTAINED PERMISSION FROM THEIR UVA PHYSICIAN:

Your doctor, Dr. insert name wanted you to be aware of this research study and gave us permission to contact you.

- We obtained your information from your medical records at UVa.

Federal regulations allow the UVa Health System to release your information to researchers at $U V a$, so that we may contact you regarding studies you may be interested in participating. We want to assure you that we will keep your information confidential.

IF THE PERSON SEEMS ANGRY, HESITANT OR UPSET, THANK THEM FOR THEIR TIME AND DO NOT ENROLL THEM IN THE STUDY. YOU MAY ALSO REFER THEM TO THE IRB-HSR AT 924-9634.

c._Direct contact of potential subjects by the study team by approaching in person at UVa or via letter, phone, direct e-mail. Members of study team contacting potential subjects ARE health care providers of patients.

If you are not approaching them in person but using a letter, phone call or direct email please note that the letter, phone, direct email scripts must be approved by IRB prior to use. See IRB-HSR Website for templates. 
DHHS: Study team requests a Waiver of Consent to contact potential subjects

HIPAA: Allowed under Health Care Operations.

d.__ X_ Indirect contact (flyer, brochure, TV, broadcast emails, patient provided info about the study from their health care provider and either the patient contacts study team or gives their healthcare provider permission for the study team to contact them.) The indirect method used (flyer, brochure, TV, broadcast emails) must be approved by the IRB prior to use. The IRB does not need to review any type of script to use when the potential subject responds to the indirect method.

DHHS \& HIPAA: NA

e. Potential subjects are not patients. The study does not include obtaining subjects health information. Subjects will be contacted directly via email, phone, letter or presentation in group setting with consent then obtained individually in a private setting.

If you are not approaching them in person but using a letter, phone call or direct email please note that the letter, phone, direct email scripts must be approved by IRB prior to use.

See IRB-HSR Website for templates.

DHHS: Study team requests a Waiver of Consent to contact potential subjects.

HIPPA: NA

\section{Will any additional information be obtained from a potential subject during "prescreening"? \\ Yes.}

IF YES, submit any documents that will be used to collect pre-screening information so that the IRB may confirm what questions will be asked. NOTE: To comply with HIPAA regulations only the minimum necessary information may be collected at this time. This means that only questions pertaining to the Inclusion and Exclusion Criteria may be asked.

\section{IF YES,}

DHHS: study team requests a Waiver of Documentation of Consent for Pre-screening questions. 
HIPPA:

- HIPAA does not apply if :

- no PHI is collected or

- if PHI is collected from a potential subject by an individual from a department that is not part of the HIPAA covered entity

- HIPAA does apply if the collection occurs by individuals* who work in a department that is part of the HIPAA covered entity. In this case the collection will be covered under Health Care Operations/

- These individuals are those that meet one of the following criteria:

- a UVa student working in the UVa HIPAA Covered Entity*

- a faculty or staff member in a PAID appointment in the UVA HIPAA Covered Entity*

IF YES, Will any of the questions involve health information? Yes.

IF YES, will you collect HIPAA identifiers with the health information?

Yes, but only if the potential subject is determined to be eligible for the study.

IF YES, which HIPAA identifiers will be recorded?

Name.

Do you confirm that health information with HIPAA identifiers will not be shared outside of UVa until a consent form is signed or only shared in a de-identified manner? Yes.

4. Do you plan to ask the subjects to do anything, other than answering questions, for the study prior to signing a consent?

No.

5. How will the consenting process take place?

HIPPA:

- If the individual, obtaining consent, works under the HIPAA Covered Entity consenting is covered under Health Care Operations.

- If the individual obtaining consent does not work under the HIPAA covered entity, HIPAA does not apply.

- Describe the setting for the consent

- How much time will the consenting process take? 
- How much time will pass between obtaining written consent and initiation of study procedures? How will you assess subject understanding?

- See Protocol Examples: Consenting Process for examples of how to answer this question.

Recruitment letters will be sent via post or email to all possible participants prior to the start of the sports season. Informational meetings will also be held for each team prior to the start of data collection. In the collegiate setting, the meetings will be attended by players, coaches, and athletic training staff. In the high school setting, the meetings will be attended by players, parents, coaches, and athletic training staff. The purpose of the meeting will be to describe the goals of the study, requirements of athletes participating in the study, timeline of the study, and enrollment procedures. Even though the schools, athletic trainers, and coaches have agreed to participate, ultimately it is the choice of every student athlete (and parent, where applicable) to choose whether to participate or not, under no penalty or pressure.

\section{For Non-Minors:}

Consent will be obtained on the day of the start of study procedures. The study is minimal risk and can be easily explained to the potential subject on the day the study procedures are started. The athlete will be interviewed in a quiet and private room of the Snyder Building in the Fontaine Research Park. If there is concern that the potential subject may not be able to read the potential subject will be asked to read the first sentence of the consent form to determine if they are capable of reading. Depending on the response they will either be offered the opportunity to read the consent form or have the consent form read to them. Once the consent has been read the person obtaining consent will summarize the consent form verbally, asking open ended questions to determine if the potential subject understands what is being covered in the consent form. Potential subjects will be given an opportunity to ask questions. Their level of understanding will dictate how much time will be spent covering each item. Once all of their questions have been answered, if they decide to participate, they will be asked to sign the consent form. The person obtaining consent will sign the form and subjects will be given a copy of the signed consent form. Study procedures will then begin.

\section{For Minors:}

Once a potential subject is identified, an IRB approved recruitment letter will be sent to the parent/guardian via post or email along with two copies of the consent form. The cover letter will require them to call the phone numbers listed in the consent form to allow the study team to discuss the study with them and answer any questions they may have. The person obtaining consent will summarize the consent form verbally and then ask open ended questions to 
determine if the potential subject understands what is being covered in the consent form. If they decide to allow the minor to participate, the Parent/Guardian will sign followed by the minor providing written assent. They will then send the signed consent back to the study team or the minor may bring the signed consent at the time of first appointment; the parents may keep the second copy for their records. Upon receiving the signed consent form back, a member of the study team who spoke with the subject on the phone will sign as the person obtaining consent.

6. Will subjects sign a consent form for any part of the study?

Subjects will sign a consent form for all parts of the study.

7. Will the study procedures be started the same day the subject is recruited for the study?

No.

8. Do you need to perform a "dry run" of any procedure outlined in this protocol?

No.

\section{APPENDIX: Participation of Children}

In the state of Virginia a person under the age of 18 is considered a child.

1. Explain why this research topic is relevant to children.

Head injuries in adolescents differ from those obtained by adults due to the continuous development they are undergoing. Thus, it is important to study the changes seen in brain acoustic monitor outcomes in children.

2. Is the knowledge being sought in this study already available for children or is it currently being acquired through another ongoing study?

The knowledge being sought in this study is not available for children and is not being acquired currently through another ongoing study.

3. Provide data that is available in adults in order that the IRB may judge the potential risk in children. If there is no adult data available, provide reasons why not. If this information is available in a sponsor's protocol, you may reference the section \# here and not duplicate the information.

The following studies have been performed at UVA in adult student athletes (participating recreationally and intercollegiately) with no adverse events: IRB \#15622, \#15871, \#16673. Also, the BAM has been used in over 1000 patients through other studies (referenced below): 
Dutton RP SJ, Aarabi B, Scalea TM. Preliminary trial of a noninvasive brain acoustic monitor in trauma patients with severe closed head injury. The Journal of Trauma Injury, Infection, and Critical Care. 2002 2002;53:857-863.

Dutton RP PK, Cohen R, Wade C, Sewell J, Fouche Y, Stein D, Arabi B, Scalea TM. Diagnosing mild traumatic brain injury: where are we now? Journal of Trauma Injury, Infection, and Critical Care. 2011;70(3):554-559.

Dutton RP, Van Der Heijden M, Aarabi B, et al. Screening TBI patients with the brain acoustic monitor: Association with CT scan findings and neurologic status at hospital discharge. Clinical Intensive Care. 2005; 16: 97-105.

Dutton RP. Noninvasive assessment of cerebral perfusion with the brain acoustic monitor. In: Vincent JL, ed. 2004 Yearbook of Intensive Care and Emergency Medicine. Berlin: Springer-Verlag; 2004:576 -586.

LaMonte MP, Sewell J, Bahouth MN, Sewell C. A noninvasive portable acoustic diagnostic system to differentiate ischemic from hemorrhagic stroke. $J$ Neuroimaging. Jan 2005;15(1):57-63.

4. Is the potential subject population likely to include wards of the state or children who are more at risk for becoming a ward of the state?

No.

4a. Is the research is this protocol related to the childs' status as a ward of the state? No

4b. Is the research to be conducted in schools, camps, hospitals, institutions, or similar settings in which the majority of children involved as subjects are not wards? Yes.

4c. Are you aware of the following requirement?

If the consent form contains a signature line for both parents the study team will notify the IRB immediately, if at any time during the course of the research, it becomes known that a potential subject is a ward of the state or that a child already enrolled in this protocol becomes a ward of the state. Yes. 
Table C2. University of Virginia Institutional Review Board Approved Consent Form $\underline{(\# 16857)}$

\section{Consent of an Adult to Be in a Research Study}

In this form "you" means a person 18 years of age or older who is being asked to volunteer to participate in this study.

\section{Parents' or Guardians' Permission for Your Child to Be in a Research Study}

\section{Agreement of a Child to Be in a Research Study Age 15 to $<18$}

In this form "you" means the child in the study and the parent or guardian.

$\checkmark$ If you are the parent or guardian, you are being asked to give permission for your child to be in this study.

$\checkmark$ If you are the child, you are being asked if you agree to be in this study.

In this form "we" means the researchers and staff involved in running this study at the University of Virginia.

In this form "you" means the person (your child) who is being asked to be in this study. As the parent or guardian, you are being asked to give permission for your child to be in this study.

\section{Participant's Name}

Principal

Investigator:

\author{
Susan Saliba \\ 210 Emmet Street South \\ Office\#209; \\ PO Box 400407 \\ Charlottesville, VA 22901 \\ Phone: 434-243-4033
}

\section{What is the purpose of this form?}

This form will help you decide if you want to be in the research study. You need to be informed about the study, before you can decide if you want to be in it. You do not have 
to be in the study if you do not want to. You should have all your questions answered before you give your permission or consent to be in the study.

Please read this form carefully. If you want to be in the study, you will need to sign this form. You will get a copy of this signed form.

\section{Who is funding this study?}

There is no funding for this study. Active Signal Technologies is providing a device (the Brain Acoustic Monitor) free of charge.

\section{Why is this research being done?}

This is a study about an experimental device called the Brain Acoustical Monitor (BAM). The BAM is a portable, non-invasive listening device that monitors blood flow in the head by listening to sound patterns on the surface of the skin. It is designed to detect and amplify sound waves created by arteries within the brain. It is similar to the stethoscope that a doctor uses to listen to your heart, except that the sound waves that it picks up are recorded and displayed on a computer. This device is not approved by the U.S. Food and Drug Administration (FDA). So far, the device has been used in more than 1000 patients some who were normal, some with mild head injuries, and some with stroke. BAM monitoring of the injured brain has been shown to help see how patients will do after having a stroke or brain injury.

Our goal is to determine how the measurements change in people who have had a concussion (mild head injury), and when you return to normal after injury using the BAM device. We want to know this because it could potentially be used to help diagnose concussions (mild traumatic brain injury) in many settings, including sports and in the military.

You are being asked to be in this study because you are a student athlete and because you are healthy with no recent head injury.

Up to 350 people will be in this study at all sites.

\section{How long will this study take?}

Your participation in this study will require 2-5 study visits over a season of athletic activity in your sport. Each visit will last about 30 minutes to 1.5 hours, depending on which session you are completing.

\section{What will happen if you are in the study?}

\section{SCREENING (will take about 5 minutes to complete):}




\section{Visit 1 (Day 1 of study participation):}

If you agree to participate, you will sign this consent form before any study related procedures take place. Before you can start in the study, there will be a screening period. You will have procedures during this time to make sure you are eligible and it is safe for you to participate. These include the following:

- Review of your medical history

If these tests show you are eligible, you will begin study treatment.

STUDY PARTICIPATION (each visit will last about 30 minutes to 1.5 hours): Day 1:

You will perform all testing in a quiet environment at Fontaine Research Park or your school's athletic training facility. We will administer a test that helps determine how well you can think and remember and take some measurements with the BAM device. All of these tests are for research purposes only.

1. You will fill out a form that will ask questions about your age, gender, education level, and whether or not you have certain disorders that may affect your performance on tests we are using in this study. This part will last approximately 5 minutes.

2. You will take a test that measures how well you are thinking and remembering. The test (SCAT3) is performed out loud and will last approximately 5 minutes.

3. To test the BAM device, a headband will be placed on your forehead and small quarter-sized sensors will be placed underneath it. A sensor will also be placed on one of your fingers using a finger clip. Then, you will be asked to sit as still and as quietly as possible while readings are taken. Each reading lasts 10 seconds and we may take up to 20 readings. The study investigator will also adjust the sensors several times throughout the BAM readings to make sure a good signal is achieved. This part of the study will take approximately 15 minutes.

\section{Days 2-5:}

If you sustain a concussion, you will participate in additional sessions several times after your injury, each lasting approximately 20 minutes. The study procedures will be the same for each of these sessions and will occur in your school's athletic training room. You will:

1. Perform the SCAT3 concussion test or just the symptom section.

2. Have a BAM reading taken.

If you do not sustain a concussion, you will participate in only one additional session after the end of the season. 
If you get a concussion:

Study Schedule8

\begin{tabular}{|c|c|c|c|c|c|c|c|c|}
\hline & $\begin{array}{l}\text { Visit 1 } \\
\text { (Screenin } \\
\text { g) }\end{array}$ & $\begin{array}{l}\text { Visit 1 } \\
\text { (Baseline }\end{array}$ & Visit 3 & Visit 4 & Visit 5 & Visit 6 & Visit 7 & Visit \\
\hline $\begin{array}{l}\text { Study } \\
\text { Week }\end{array}$ & $\begin{array}{l}\text { Preseaso } \\
\text { n (Day } \\
1 \text { ) }\end{array}$ & $\begin{array}{l}\text { Preseas } \\
\text { on (Day } \\
1 \text { ) }\end{array}$ & $\begin{array}{l}\text { In- } \\
\text { season } \\
\text { (Day 2) } \\
\end{array}$ & $\begin{array}{l}\text { In- } \\
\text { season } \\
\text { (Day 3) }\end{array}$ & $\begin{array}{l}\text { In- } \\
\text { season } \\
\text { (Day 4) } \\
\end{array}$ & $\begin{array}{l}\text { In- } \\
\text { season } \\
\text { (Day 5) } \\
\end{array}$ & $\begin{array}{l}\text { In- } \\
\text { season } \\
\text { (Day 6) }\end{array}$ & $\begin{array}{l}\text { End of } \\
\text { Season } \\
\text { (Day 6) }\end{array}$ \\
\hline $\begin{array}{l}\text { Informed } \\
\text { Consent }\end{array}$ & $X$ & & & & & & & \\
\hline $\begin{array}{l}\text { Review } \\
\text { study } \\
\text { eligibility }\end{array}$ & $\mathrm{X}$ & & & & & & & \\
\hline $\begin{array}{l}\text { Medical } \\
\text { History }\end{array}$ & $\mathrm{x}$ & & & & & & & \\
\hline $\begin{array}{l}\text { Memory } \\
\text { Test (full } \\
\text { SCAT3) }\end{array}$ & & $\mathrm{X}$ & & & & $\mathrm{X}$ & & $\mathrm{X}$ \\
\hline $\begin{array}{l}\text { Symptom } \\
\text { Inventory } \\
\text { (using } \\
\text { SCAT3) }\end{array}$ & & & $\mathrm{x}$ & $\mathrm{X}$ & $\mathrm{X}$ & & $\mathrm{X}$ & \\
\hline $\begin{array}{l}\text { BAM } \\
\text { device } \\
\text { Measureme } \\
\text { nt }\end{array}$ & & $\mathrm{X}$ & $\mathrm{X}$ & $\mathrm{X}$ & $\mathrm{X}$ & $\mathrm{X}$ & $X$ & $\mathrm{x}$ \\
\hline
\end{tabular}

If you want to know about the results before the study is done:

During the study you are having an investigational test done. The purpose of the test is not to diagnose any disease or abnormality you may have. Because the test is investigational there is no way for the study leader to understand if the results are "normal" or "abnormal". However, if any test results are concerning, your study leader will let you know.

The final results of the research will not be known until all the information from everyone is combined and reviewed. At that time you can ask for more information about the study results.

\section{What are the risks of being in this study?}

Risks and side effects related to the study procedures include: 


\section{Less Likely}

- Temporary worsening of the symptoms associated with concussion such as headache, dizziness, fatigue, or difficulty concentrating. These symptoms can worsen with mental exercise of the study test.

\section{Other unexpected risks:}

You may have side effects that we do not expect or know to watch for now. Call the study leader if you have any symptoms or problems.

\section{Could you be helped by being in this study?}

You will not benefit from being in this study. However the information researchers get from this study may help others in the future.

\section{What are your other choices if you do not join this study?}

You do not have to be in this study to be treated for your illness or condition. You can get the usual treatment even if you choose not to be in this study. The usual treatment would include the follow-up computer test that you take after having a concussion. This is used to monitor your recovery and make a decision about your return to athletic participation.

If you are an employee of UVa your job will not be affected if you decide not to participate in this study.

If you are a student at UVa, Charlottesville High School, St. Anne's Belfield School, Longwood University, The Covenant School, or Western Albemarle High School, your grades will not be affected if you decide not to participate in this study.

\section{Will you be paid for being in this study?}

You will not get any money for being in this study.

\section{Will being in this study cost you any money?}

The following procedures/tests, which are being done for research purposes, will be provided at no cost to you or your health insurance: Brain Acoustic Monitor testing and the paper and pencil tests. You will be responsible for the cost of travel to come to any study visit and for any parking costs.

\section{What if you are hurt in this study?}

If you are hurt as a result of being in this study, there are no plans to pay you for medical expenses, lost wages, disability, or discomfort. The charges for any medical treatment you receive will be billed to your insurance. You will be responsible for any amount your 
insurance does not cover. You do not give up any legal rights, such as seeking compensation for injury, by signing this form.

\section{What happens if you leave the study early?}

You can change your mind about being in the study any time. You can agree to be in the study now and change your mind later. If you decide to stop, please tell us right away. You do not have to be in this study to get services you can normally get at the University of Virginia.

Even if you do not change your mind, the study leader can take you out of the study.

If you decide to stop being in the study, we will ask you to write to the prinicipal investigator or call to inform her that you would like to withdraw:

Susan Saliba, $\mathrm{PhD}$

210 Emmet Street South

Office\#209; PO Box 400407

Charlottesville, VA 22901

Phone: 434-243-4033

\section{How will your personal information be shared?}

The UVa researchers are asking for your permission to gather, use and share information about you for this study. If you decide not to give your permission, you cannot be in this study, but you can continue to receive regular medical care at UVA.

\section{If you sign this form, we may collect any or all of the following information about you:}

1. Personal information such as name, address, date of birth,

2. Social Security number only if you are being paid to be in this study

3. Your health information. If required for this study, this may include a review of your medical records and test results from before, during and after the study from any of your doctors or health care providers.

\section{Who will see your private information?}

- The researchers to make sure they can conduct the study appropriately, observe the effects of the study and understand its results

- People or committees that oversee the study to make sure it is conducted correctly

- People who pay for the study, including insurance companies

- Tax reporting offices (if you are paid for being in the study)

- People who evaluate study results, which can include sponsors and other companies that make the drug or device being studied, researchers at other sites conducting the 
same study, and government agencies that provide oversight such as the Food and Drug Administration (FDA) if the study is regulated by the FDA.

The information collected from you might be published in a medical journal. This would be done in a way that protects your privacy. No one will be able to find out from the article that you were in the study.

\section{What if you sign the form but then decide you don't want your private information shared?}

You can change your mind at any time. Your permission does not end unless you cancel it. To cancel it, please send a letter to the researchers listed on this form. Then you will no longer be in the study. The researchers will still use information about you that was collected before you ended your participation. UVa researchers will do everything possible to protect your privacy.

However, they will need to share your information with people outside of UVa who may not have to follow the federal Privacy Rule. Some of those people may be allowed to share/release your information without your permission.

\section{Please contact the researchers listed below to:}

- Obtain more information about the study

- Ask a question about the study procedures or treatments

- Report an illness, injury, or other problem (you may also need to tell your regular doctors)

- Leave the study before it is finished

- Express a concern about the study

Susan Saliba, PhD

210 Emmet Street South

Office\#209; PO Box 400407

Charlottesville, VA 22901

Phone: 434-243-4033

\section{What if you have a concern about a study?}

You may also report a concern about a study or ask questions about your rights as a research subject by contacting the Institutional Review Board listed below.

University of Virginia Institutional Review Board for Health Sciences Research PO Box 800483

Charlottesville, Virginia 22908 
Telephone: 434-924-2620

When you call or write about a concern, please give as much information as you can. Include the name of the study leader, the IRB-HSR Number (at the top of this form), and details about the problem. This will help officials look into your concern. When reporting a concern, you do not have to give your name.

\section{Signatures}

\section{What does your signature mean?}

Before you sign this form, please ask questions about any part of this study that is not clear to you. Your signature below means that you have received this information and all your questions have been answered. If you sign the form it means that you agree to join the study. You will receive a copy of this document after you have signed it.

\section{Consent From Adult}

PARTICIPANT

(SIGNATURE)
PARTICIPANT

(PRINT)
DATE

\section{Person Obtaining Consent}

By signing below you confirm that you have fully explained this study to the potential subject, allowed them time to read the consent or have the consent read to them, and have answered all their questions.

PERSON OBTAINING CONSENT (SIGNATURE)
PERSON OBTAINING CONSENT (PRINT)

\section{Assent from Child}

Consent from the parent/guardian MUST be obtained before approaching the child for their assent.

PARTICIPANT (SIGNATURE)
PARTICIPANT

(PRINT)
DATE 


\section{Person Obtaining Assent of the Child}

Consent from the parent/guardian MUST be obtained before approaching the child for their assent.

By signing below you confirm that the study has been explained to the child (less than 18 years of age), all questions have been answered and the child has voluntarily agreed to participate.

PERSON OBTAINING

ASSENT

(SIGNATURE)

\section{PERSON OBTAINING \\ DATE}

ASSENT

(PRINT)

\section{Parental/ Guardian Permission}

By signing below you confirm you have the legal authority to sign for this child.

PARENT/GUARDIAN (SIGNATURE)

$\overline{\text { PARENT/GUARDIAN }} \quad \overline{\text { DATE }}$
(PRINT NAME)

\section{Person Obtaining Parental Permission}

By signing below you confirm that you have fully explained this study to the parent/guardian, allowed them time to read the consent or have the consent read to them, and have answered all their questions.

PERSON OBTAINING

PARENTAL PERMISSION

(SIGNATURE)
PERSON OBTAINING

$\overline{\mathrm{DATE}}$ 8 
Table C3. University of Virginia Institutional Review Board Approved Assent Form

Participant's Name

\section{Medical Record \#}

\section{ASSENT FORM TO BE IN A RESEARCH STUDY}

Doctors at the University of Virginia are trying to learn more about concussions and what they do to blood flow in your brain. This is called a research study.

The reason to do this research study is because we want to be able to tell if you have a concussion and when it is healed so that you are safe when you start playing sports again.

You are asked to be in this research study because you play sports at your high school.

This is what will happen during this study:

You will take a test that will let us see how well you can think and remember things. This only takes about 5 minutes.

You will also get measured using a tool called the BAM. This allows us to measure the blood flow in your brain. It does not hurt and you don't feel anything at all while it's working. We will just attach two little sensors that are shaped like quarters to your head using a strap. This only takes about 10 minutes.

If you have a concussion sometime while you are playing sports at your high school this season, you will get these tests repeated up to 5 times. Each time will only take about 30 minutes.

If you don't have a concussion sometime while you are playing sports at your high school this season, you will only repeat these tests once. This will happen when the season is over.

Sometimes things happen to people in research studies that may hurt them or make them feel bad. These are called risks. The risks of this study are making concussion symptoms like headache, stomache ache, or dizziness worse because of the testing. This is very uncommon, though.

If you are pregnant or think you might be pregnant please tell us so we may talk about this with you.

You do not have to be in this study if you do not want to be. 
You may stop being in the study at any time. If you decide to stop, no one will be angry or upset with you.

You will not receive anything for being in this research study.

Please ask as many questions as you need to make sure you understand the study before you sign this form.

\section{$\underline{\text { Assent from Child }}$}

$\begin{array}{lll}\begin{array}{l}\text { CHILD } \\ \text { (SIGNATURE) }\end{array} & \overline{\text { CHILD }} \\ \text { (PRINT NAME) } & \overline{\text { DATE }}\end{array}$

Consent from the parent/guardian MUST be obtained before approaching the child for their assent. If the child is not able to read English, the minor should not sign this form. There should be written documentation in the study file noting that study was explained to the child, all questions were answered and the child verbally agreed to participate in the study.

\section{Person Obtaining Assent of the Child}

By signing below you confirm that the study has been explained to the child (less than 18 years of age), all questions have been answered and the child has voluntarily agreed to participate.

\section{PERSON OBTAINING ASSENT OF THE CHILD \\ (SIGNATURE)}

\section{PERSON OBTAINING ASSENT OF THE CHILD (PRINT NAME)}

DATE

\section{Parental/ Guardian Permission}

By signing below you confirm you have the legal authority to sign for this child.

\section{PARENT/GUARDIAN}

(SIGNATURE)
DATE

\section{Person Obtaining Parental Permission}

By signing below you confirm that you have fully explained this study to the parent/guardian, allowed them time to read the consent or have the consent read to them, and have answered all their questions.

PERSON OBTAINING PARENTAL PERMISSION (SIGNATURE)

\begin{tabular}{l}
\hline PERSON OBTAINING \\
PARENTAL PERMISSION \\
(PRINT NAME)
\end{tabular}

DATE 
$\underline{\text { Table C4. Patient Medical History Form for Reliability Study (Manuscript I) }}$

Subject ID: IRB\#

\begin{tabular}{|c|c|c|c|c|c|}
\hline \multicolumn{2}{|c|}{ HEIGHT } & SEIGHT & & AGE & DATE OF BIRTH \\
\hline & & & $/$ \\
\hline
\end{tabular}

What is your dominant hand?

Right $\square$ Left

Which leg would you use to kick a ball?

Right $\square \quad$ Left

Which leg would you use to jump from?

Right

Left

Please check below if you have or have had any of the following:

General Medical

Allergies (latex, heat, cold, electricity, medications, etc)

Cancer

$\square$ Biomedical Devices (implants, pacemaker)

Currently pregnant or nursing

Recent illness (upper respiratory infection, cold, infections)

$\square$ Diabetes

Asthma

Surgery

Other:

Please explain any checked items:

Please provide date of last physical exam:

Neurological

$\square$ Epilepsy/seizures

$\square$ Anxiety disorders

ADHD

Diabetic Neuropathy

$\square$ Concussion OR Traumatic Brain

Injury

Please explain any checked items:

\section{Cardiovascular}

$\square$ Stroke

High Blood Pressure

Heart Attack

Shortness of Breath

Sickle Cell Trait

Heart Murmur $\square$ Cerebral Palsy

Balance Disorder

Vertigo

Parkinson's Disease

$\square$ Multiple Sclerosis

Other 
Please explain any checked items:

\section{General Orthopedic}
$\square$ Rheumatoid Arthritis
Osteoarthritis
$\square$ Osteoporosis/Osteopenia
Previous Fracture
Surgery
Gout
Assistive Devices (crutches, braces)
Other:

Please explain any checked items:

\section{Other}

Have you taken any prescription or over the counter medications within the last 24 hours?

Yes No

If yes, please list:

Have you consumed any of the following stimulants or depressants in the last 12 hours?

$\square$ Caffeine

Tobacco

Alcohol

Other

If yes, please explain:

Do you exercise regularly?

Yes No

If yes, how often, what type and for how long?

Are you currently on an athletic team?

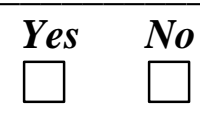

If yes, at what level?

If yes, for what sport? 
Table C5. Exercise History Questionnaire for Reliability Study (Manuscript I)

\title{
GODIN LEISURE-TIME EXERCISE QUESTIONNAIRE
}

1. During a typical 7-Day period (a week), how many times on the average do you do the following kinds of exercise for more than $\mathbf{1 5}$ minutes during your free time (write on each line the appropriate number).

\section{Times Per \\ Week}

\author{
a) STRENUOUS EXERCISE (HEART BEATS RAPIDLY) \\ (e.g., running, jogging, hockey, football, soccer, \\ squash, basketball, cross country skiing, judo, \\ roller skating, vigorous swimming, \\ vigorous long distance bicycling) \\ b) MODERATE EXERCISE (NOT EXHAUSTING) \\ (e.g., fast walking, baseball, tennis, easy bicycling, \\ volleyball, badminton, easy swimming, alpine skiing, \\ popular and folk dancing)
}

\section{c) MILD EXERCISE (MINIMAL EFFORT)}

(e.g., yoga, archery, fishing from river bank, bowling, horseshoes, golf, snow-mobiling, easy walking)

2. During a typical 7-Day period (a week), in your leisure time, how often do you engage in any regular activity long enough to work up a sweat (heart beats rapidly)?

Sometimes

Often

Rarely 


\section{GRADING INSTRUCTIONS}

The individual is asked to complete a self-explanatory, brief four-item query of usual leisure-time exercise habits.

\section{CALCULATIONS}

For the first question, weekly frequencies of strenuous, moderate, and light activities are multiplied by nine, five, and three, respectively. Total weekly leisure activity is calculated in arbitrary units by summing the products of the separate components, as shown in the following formula:

Weekly leisure activity score $=(9 \times$ Strenuous $)+(5 \times$ Moderate $)+(3 \times$ Light $)$

The second question is used to calculate the frequency of weekly leisure-time activities pursued "long enough to work up a sweat" (see questionnaire).

\section{EXAMPLE}

Strenuous $=3$ times $/$ wk

Moderate $=6$ times $/ \mathrm{wk}$

Light $=14$ times $/$ wk

Total leisure activity score $=(9 \times 3)+(5 \times 6)+(3 \times 14)=27+30+42=99$ 
Table C6. Data Collection Sheet for Reliability Study

Day 1:

\begin{tabular}{|c|c|c|c|c|}
\hline Trial: & $\begin{array}{c}\text { Heart } \\
\text { Rate }\end{array}$ & BP & Assessor \#1 & Assessor \#2 \\
\hline 0 & & & & \\
\hline 1 & & & & \\
\hline 2 & & & & \\
\hline 3 & & & & \\
\hline 4 & & & & \\
\hline
\end{tabular}

Day 2:

\begin{tabular}{|c|c|c|l|}
\hline Trial: & $\begin{array}{c}\text { Heart } \\
\text { Rate }\end{array}$ & BP & Assessor \#1 \\
\hline 0 & & & \\
\hline 1 & & & \\
\hline 2 & & & \\
\hline 3 & & & \\
\hline 4 & & & \\
\hline
\end{tabular}

\begin{tabular}{|c|l|l|l|}
\hline \multicolumn{3}{|c|}{ Exercise Protocol } \\
\hline$\underline{\text { Time: }}$ & $\underline{\text { HR }}$ & $\underline{\text { BORG }}$ & Speed \\
\hline 0 mins & & & \\
\hline 5 mins & & & \\
\hline 7 mins & & & \\
\hline 9 mins & & & \\
\hline 11 mins & & & \\
\hline 13 mins & & & \\
\hline 15 mins & & & \\
\hline
\end{tabular}




\begin{tabular}{|c|l|l|l|}
\hline 17 mins & & & \\
\hline 19 mins & & & \\
\hline
\end{tabular}

\begin{tabular}{|c|c|c|l|}
\hline Trial: & $\begin{array}{c}\text { Heart } \\
\text { Rate }\end{array}$ & BP & Assessor \#1 \\
\hline 0 & & & \\
\hline 1 & & & \\
\hline 2 & & & \\
\hline 3 & & & \\
\hline 4 & & & \\
\hline
\end{tabular}


Table C7. Data Collection Sheet for Concussion Study

Subject \#:

\section{HEIGHT}

WEIGHT

SEX

AGE

DATE OF BIRTH

$\mathrm{ft}$.

lbs.

yrs.

/ I

What is your dominant hand?

Right

Left

Do you have a learning disability?

Yes

No

ADD/ADHD?

Yes

No

Current level of education: $\square 1^{\text {st }}$ year $\square 2^{\text {nd }}$ year $\square 3^{\text {rd }}$ year $\square 4^{\text {th }}$ year $\square$ Graduate Concussion History:

Date of last concussion:

No. of concussions experienced:

Time since last concussion:

Do you have any medical condition or neurological disorders that might affect balance or cognition? Yes $\square$ No $\square$

If so, please list here:

Baseline neurocognitive test scores available: $\quad$ Yes $\square$ No $\square$

Do you participate in athletics at UVA, St. Anne's Belfield School, or Charlottesville High School? Yes $\square$ No $\square$ 
Table C8. Sideline Concussion Assessment Tool, version 3(SCAT3).

\section{SCAT3 $^{\text {tw }}$}

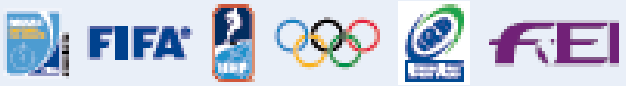

\section{Sport Concussion Assessment Tool - 3rd Edition}

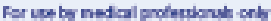

Haxe

\section{What is the SCAT3?}

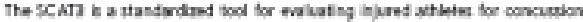

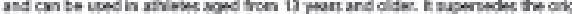

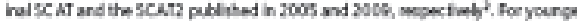

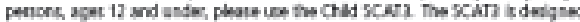

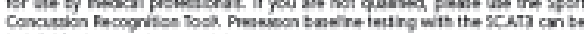

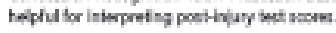

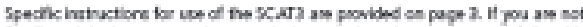

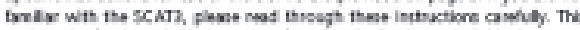

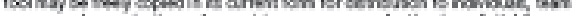

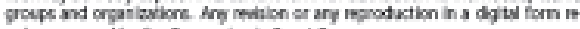

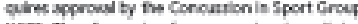

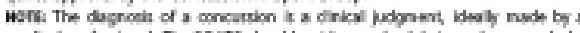

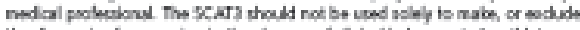

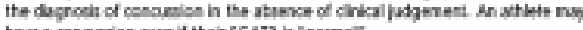

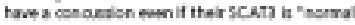

What is a concussion?

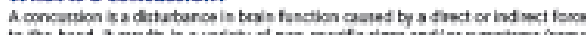

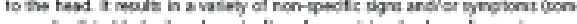

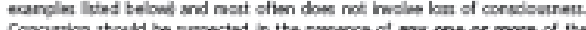

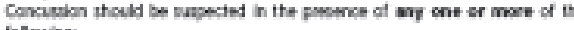
falowing

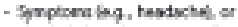

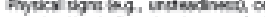

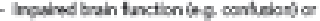

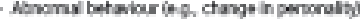

\section{SIDELINE ASSESSMENT}

Indications for Emergency Management

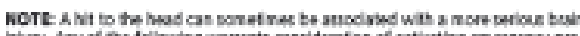

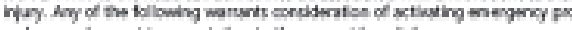

Congow Cora some kes fun is

Detwiosving restivi ritat

- Povertial pingi injay

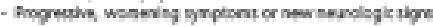

Potential signs of concussion?

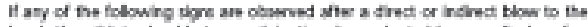

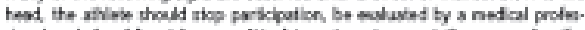
thond and thould aos be permitied to neturn to epert the seme dinf ifs

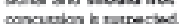

Any lise of coreciournes:

"Ens, kow lang"

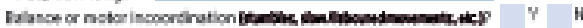

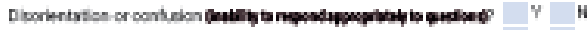

Lout of menom:

"Ens, kow lang"

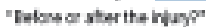

Ebri or want bod:

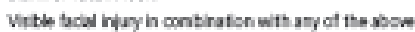

Glasgow coma scale (GCS)

aest epe respones (I)

Nowerning

Ipoparing in mespone is pain

Epopking to resect

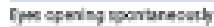

aest wertal recpana (V)

Ko vetod newpens.

Inerenguturituls sound:

insporprits morts

Centrest

Criertes

aest metor retponces fin

No motar mponte

Falurelon top puin

Akromd fedon io pain

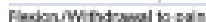

Lecalms to pain

Cbaps connind

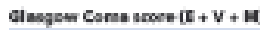

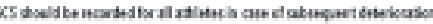

\section{Maddocks Score}

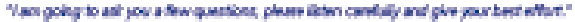

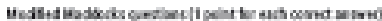

What veves ne we st bate?

Which hell is it nowl

Who wored last in sit nuph?

What sean dit peo ply bat membignes

Did your tase win the last gavet $\quad 0 \quad 1$

Moddoda ware of

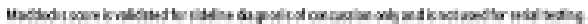

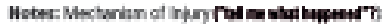

Any athlete with a susected cencusaion should be REMOVtD Wome hLAY, medically assessed, monibored for deterioration (i.-, should not be lett alone) and should not dehes a motor wehide until cleared to do so by a medical protessional Wo athiote diag. neased with concusaisen should be returaed to sports participation on the dey of lajury. 


\section{BACKGROUND}

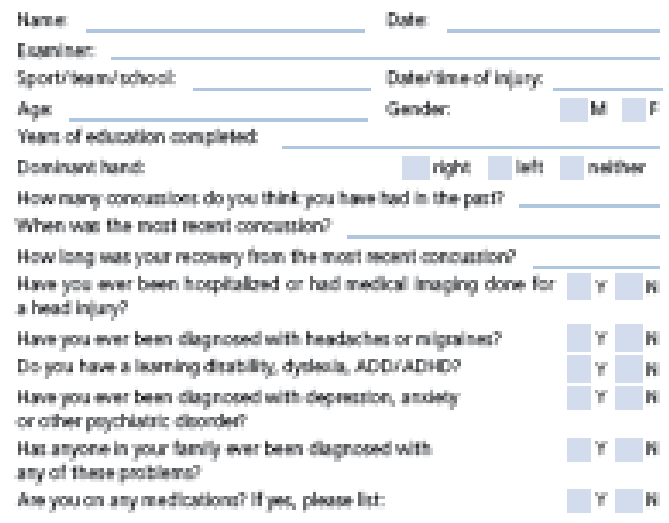

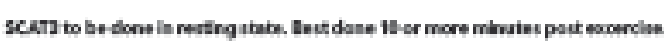
SYMPTOM EVALUATION

\section{How do you feel?}

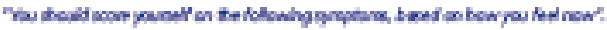

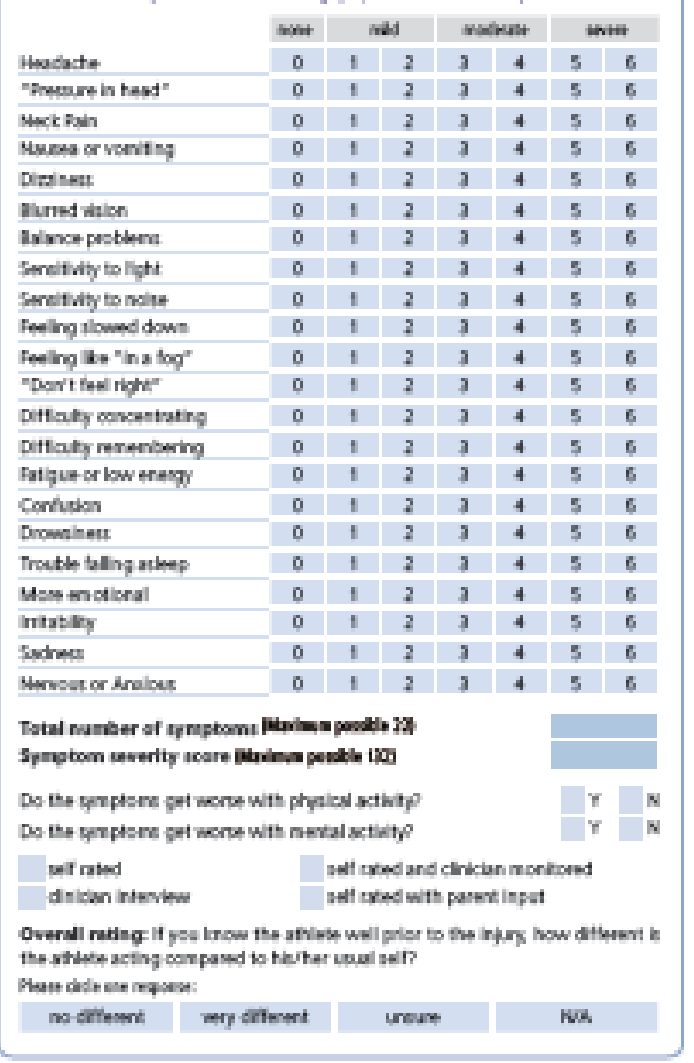

Scoring en the SCArrs should not be uned wa atand-alone method to alngnose concunaiss, messure recovery or make decinisns about an athleteri neadineas bo metum to competitien after concusaisn. Since signs and symptoms may evolos over time, it is important to conaider mepeat evaluation in the acuts asessment of cencuasion.

\section{COGNITIVE \& PHYSICAL EVALUATION}

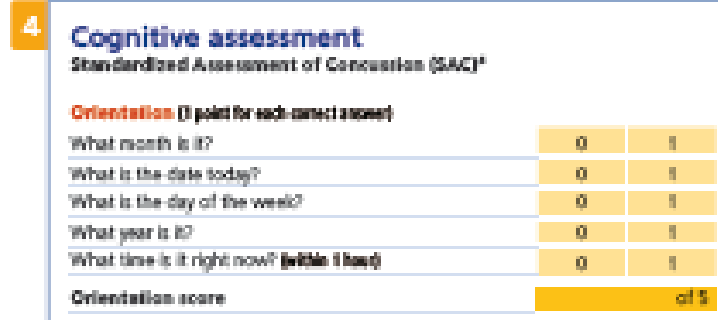

Imunediake nemery

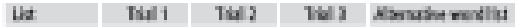

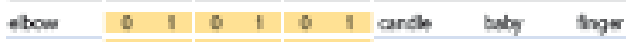

apple o 1 i o 1 | o 1 peor nork perres

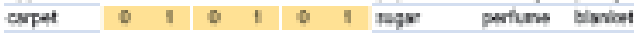

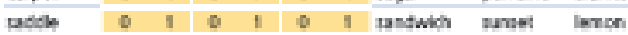

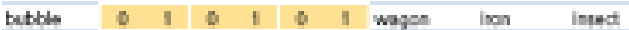

Toed

Inmedive menary weare teted

Cancwatration: Degita Eachward

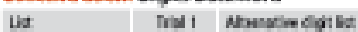

$\begin{array}{llllll}4-9-3 & 0 & 1 & 6-2.9 & 5.2-6 & 4-1.5\end{array}$

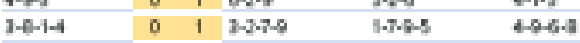

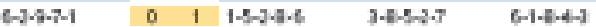

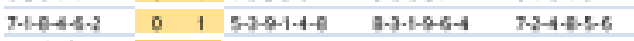

Total of 4

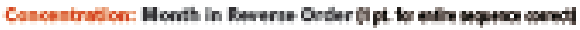

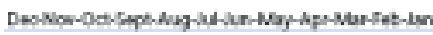

0

Cancentration scers

\section{Neck Examination:}

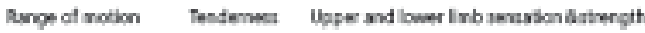

Andinge

\section{Balance examination}

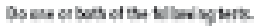

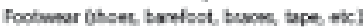

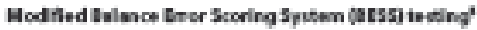

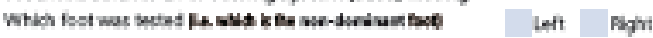

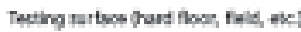

Conalian

Doublikg stunce

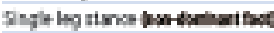

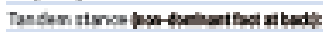

And/Or

Twadem gaite"

Trut Buted daint weond

\section{Coordination examination}

Uaper ambecorthatien

Wevich sm was inted: Left Dope

Coocthation were

SAC Delayed Recall ${ }^{4}$

Delayed recell scors 


\section{INSTRUCTIONS}

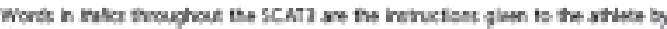
the tetie:

\section{Symptom Scale}

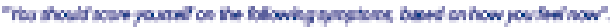

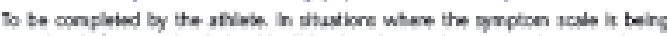

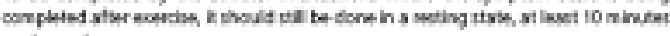
poet enancis

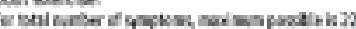

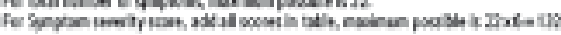

SAC

Immedlive Nemery

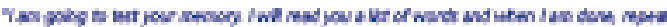

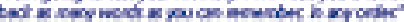

Triala 2 as:

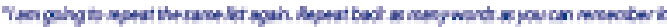

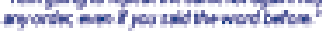

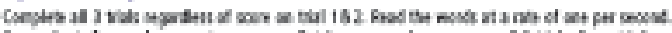

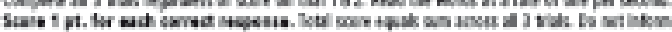

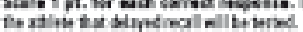

\section{Cancentrabion}

Dights bediwind

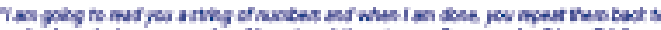

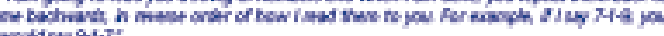
wodidedy $91-x^{2}$

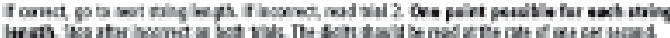

Nonthi in wewerse onder

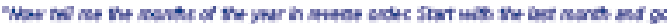

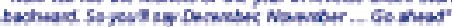

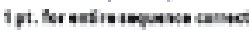

Delinyed Ancul|

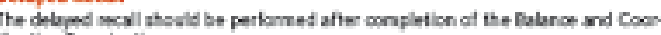
dination fiaviration.

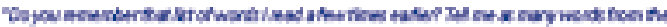

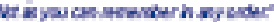

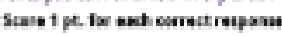

\section{Balance Examination}

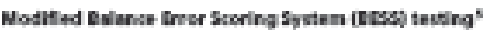

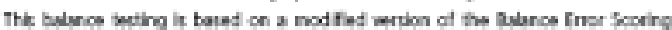

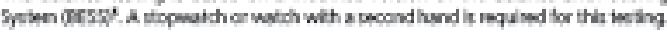

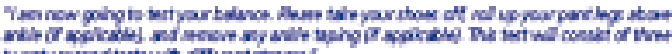

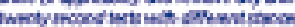

(a) Deutle leg atance:

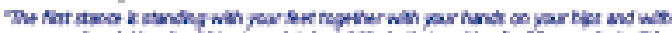

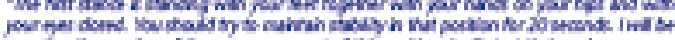

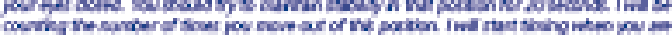

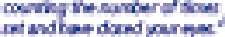

(b) single leg ntance:

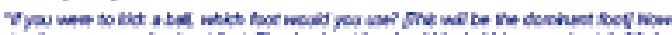

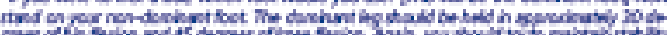

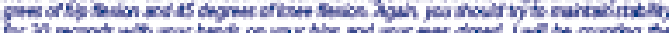

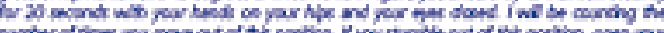

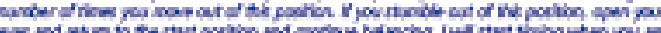

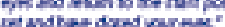

\section{(c) Tandere tarke:}

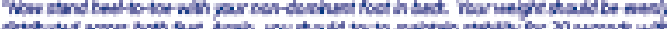

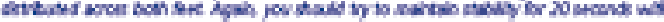

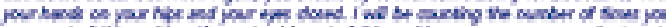

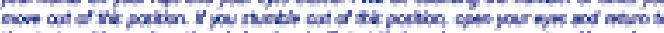

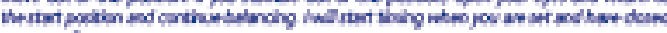
pourtye:
Dalance torting - tppen of errars

1. Hande lined eff lax onet

20 opering epet

3.3 sop stumble or tis

4. Moving hip inbo $>>0$ degness saduction

5. Ining tand oct or hem

6. Anvining out ef tast potison $>5$ sec

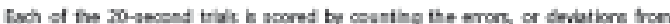

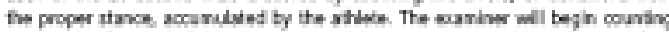

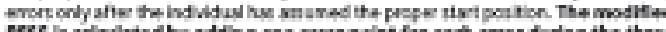

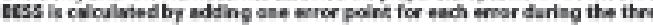

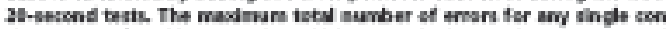

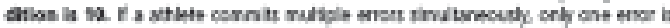

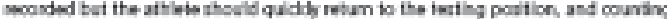

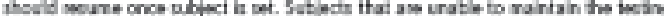

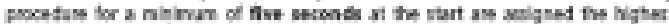

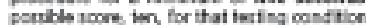

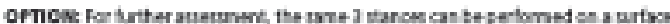

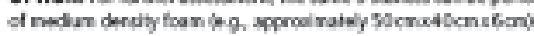

\section{Tondem Quiter}

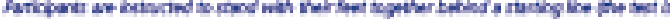

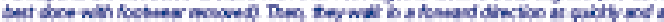

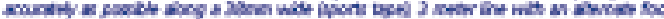

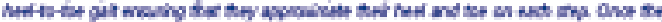

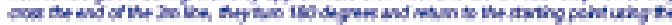

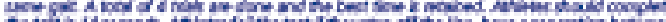

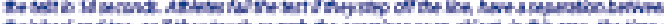
cose ntetion

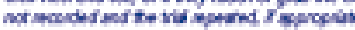

\section{Coordination Examination}

\section{Uaper Irib coardination}

Anom-bonate opto tat:

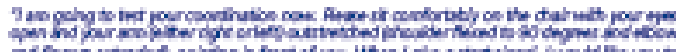

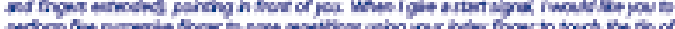

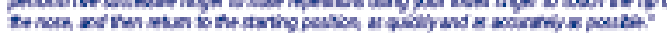

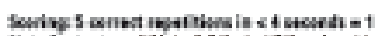

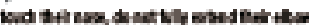

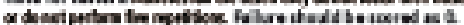

\section{References \& Footnotes}

1. Thit bol hat been developed by a group of irienutional experts at the ith is.

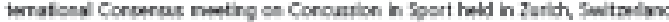
in Nownter 2012 the fal cetult of the conlerenco oukeres and the surtors of

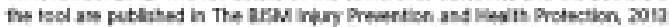

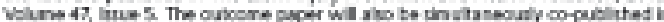

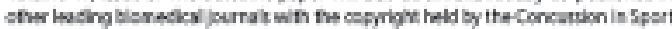

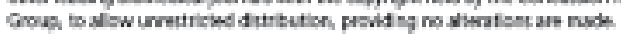

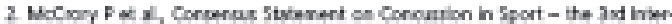

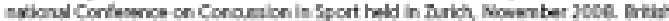
bound ef Spertt Wackins 2009 43: De-60.

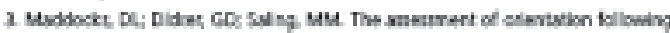

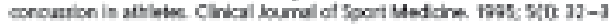

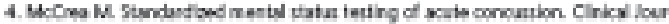

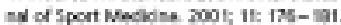

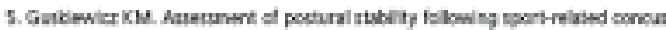

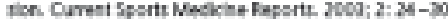

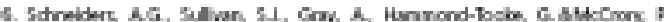

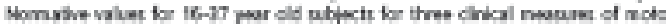

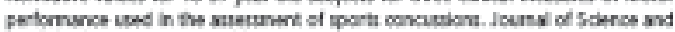

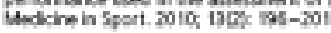

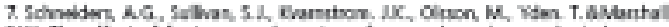

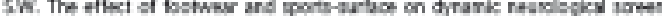

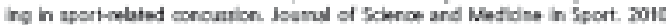
18ve $302-306$ 


\section{ATHLETE INFORMATION}

Any whllele suspected of having a concusion should be nemoved trom play, and then seek, medical enaluation.

\section{Signs to watch for}

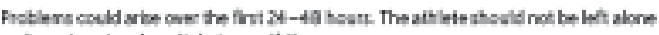
and nat 90 bo a hoeplid st ence if they

- Hawe a hededache thac gets worne

- Ace very drowir ar cin't be inviluened

- Cin't recogrias pesple or placen

- Howe repealed womiting

- Behive unatually or neen confuned ane wery intable

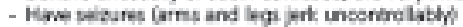

- Howe wask or numb arms or legs

- Ane ursteady on ther feet, have sumed speech

Remenber, it is better to be ante.

Conault your doctoe after a suipected cencuasion.

\section{Return to play}

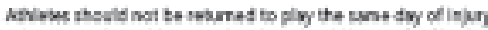

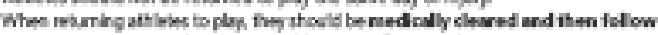

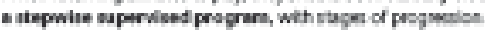

\section{For example:}

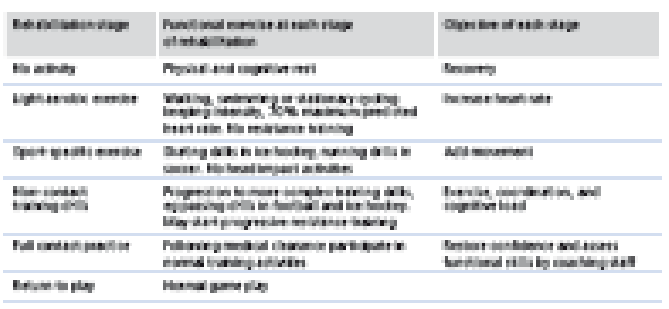

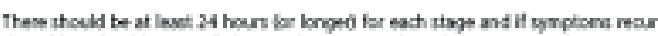

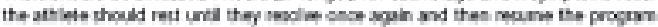

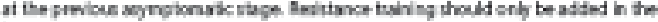
bater tapen.

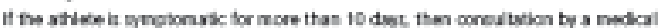

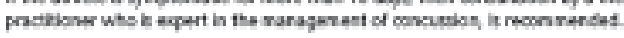

Medical dearince ahould be given before return to pley.

\begin{tabular}{|c|c|c|c|}
\hline \multicolumn{4}{|c|}{ Scoring Summary: } \\
\hline \multirow[t]{2}{*}{ Teet Donvin } & \multicolumn{3}{|c|}{ Soane } \\
\hline & Das: & Das: & Drex: \\
\hline \multicolumn{4}{|c|}{ Number of Sy rptons of 22} \\
\hline \multicolumn{4}{|c|}{ Spupten Swurky Socore of 132} \\
\hline \multicolumn{4}{|l|}{ Cripriation ofs } \\
\hline \multicolumn{4}{|l|}{ innedvat Whencoy of is } \\
\hline \multicolumn{4}{|l|}{ Cencertustion ats } \\
\hline \multicolumn{4}{|c|}{ Detyot Pecal of 5} \\
\hline \multicolumn{4}{|c|}{ SACTotel } \\
\hline \multicolumn{4}{|l|}{ erss tactal wreero } \\
\hline \multicolumn{4}{|l|}{ Tenden Gat besondes } \\
\hline Ceontration of I & & & \\
\hline
\end{tabular}

Notes:

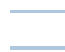

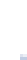

\section{CONCUSSION INJURY ADVICE \\ top to ghen is the perion meniboring the cenaras stives}

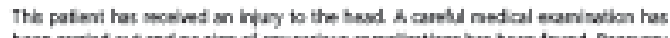

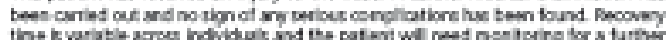

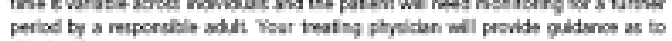
the freptune.

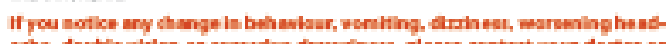

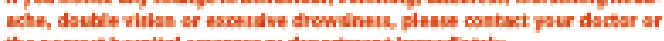
the newest houplial emerguacy department imonediabely.

\section{Cher inportant painte}

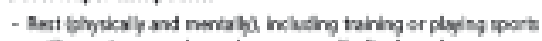

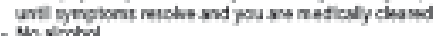

- Mo vicothal

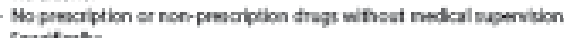
Specticaly:

No temping twolies

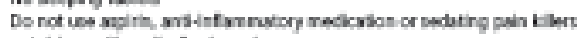

- Do nst arim unfi meficaly clemes

- Do not trin or ply port unili nad cally claved

Clinic phone numbet

Batiert'rnams

Date/tinestirikn

Dateitine st resticd smian

Trevingrbydicin

Cotat biturartary 
Table C9. Process for creating patient file on BAM.

1. Open BAM application. (Figure $\mathrm{C} 1$ )

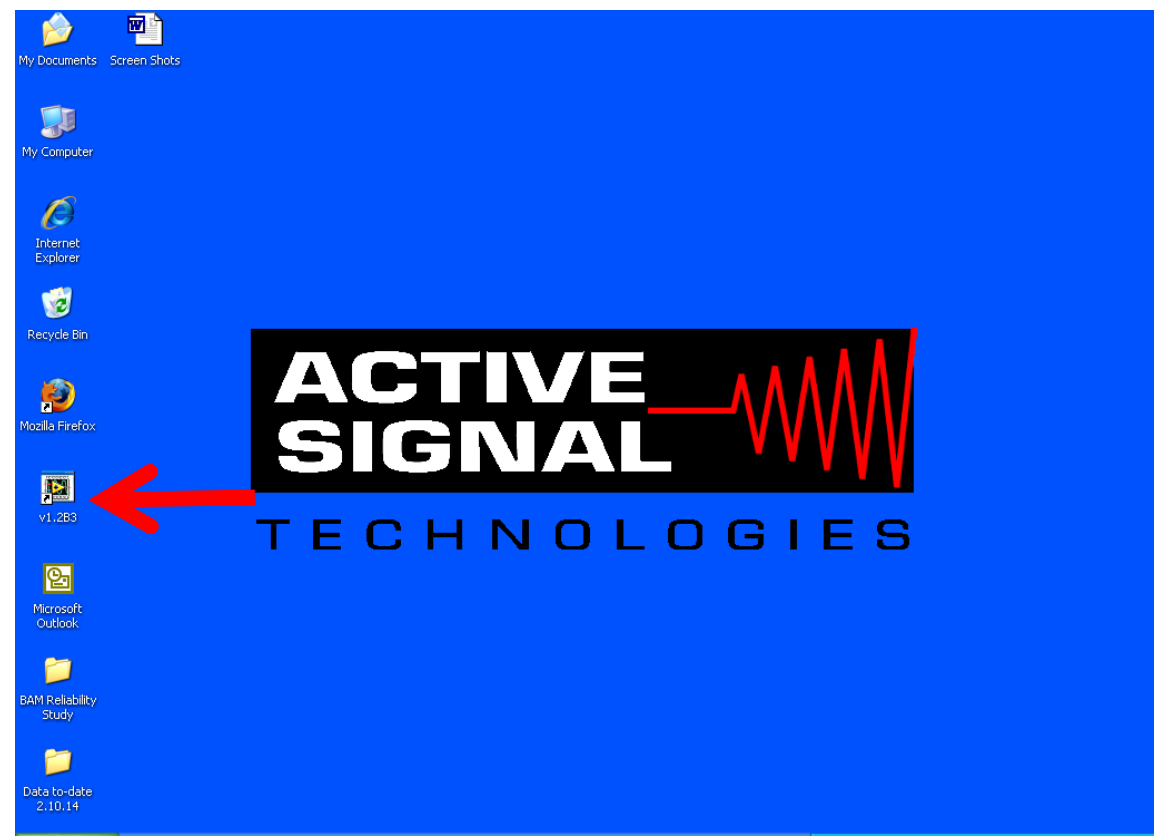

Figure C1.

2. In "patient selector" menu, select, add "add new patient". (Figure C2)

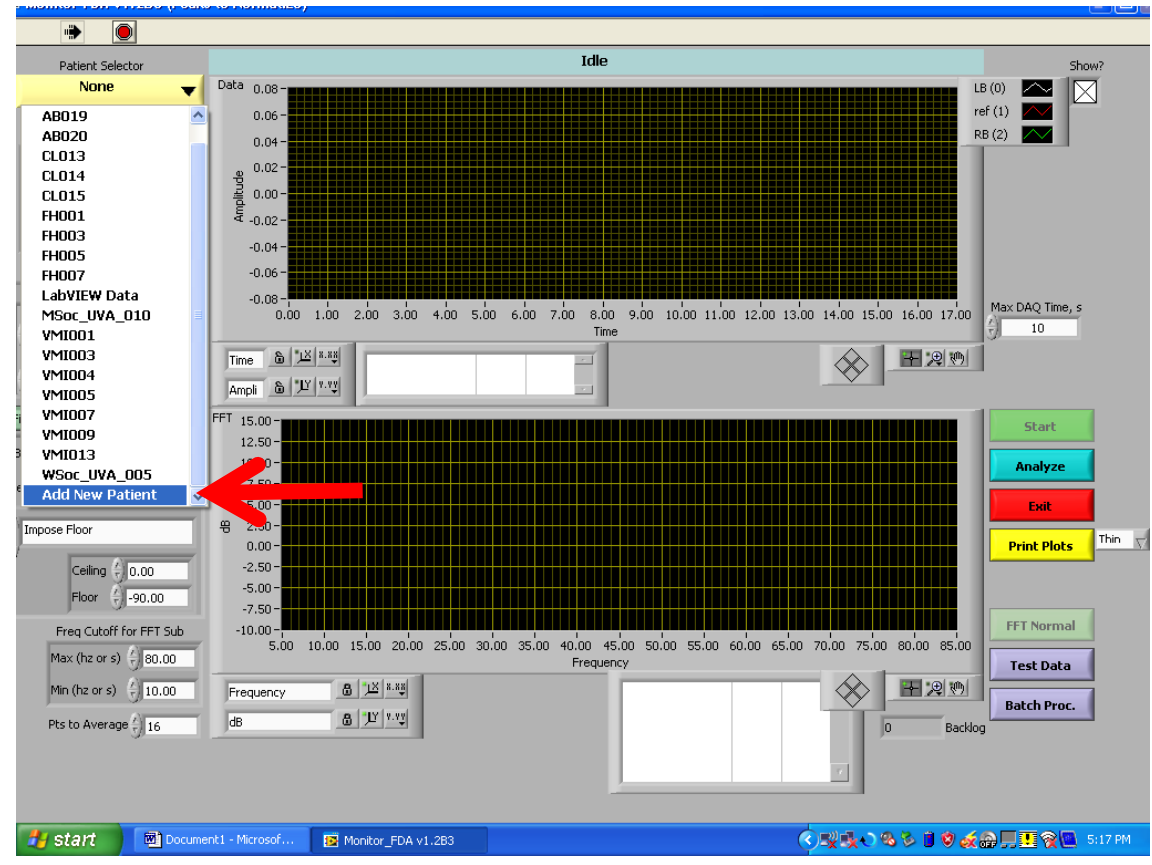

Figure C2. 
3. Enter in appropriate patient ID. Click ok. (Figure C3)

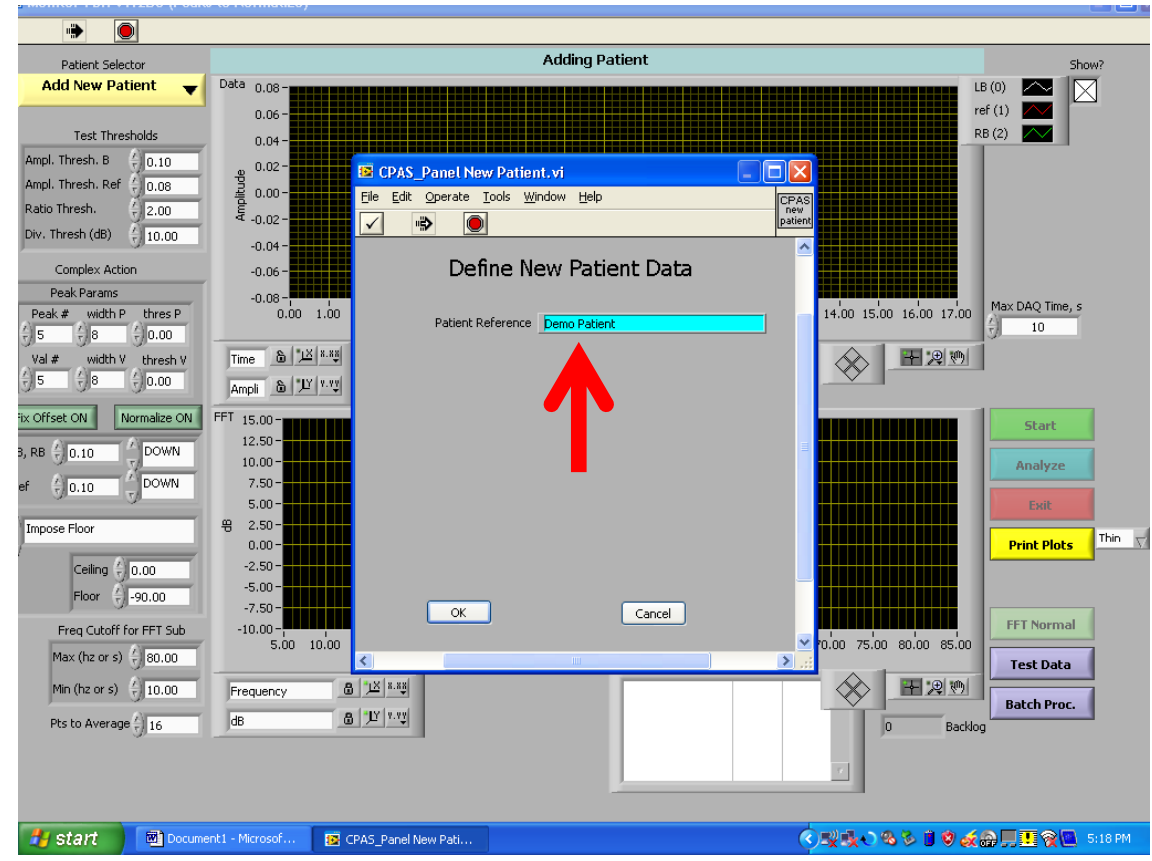

\section{Figure C3.}

4. Re-click "select patient". In the drop down menu, click newly added patient. (Figure C4)

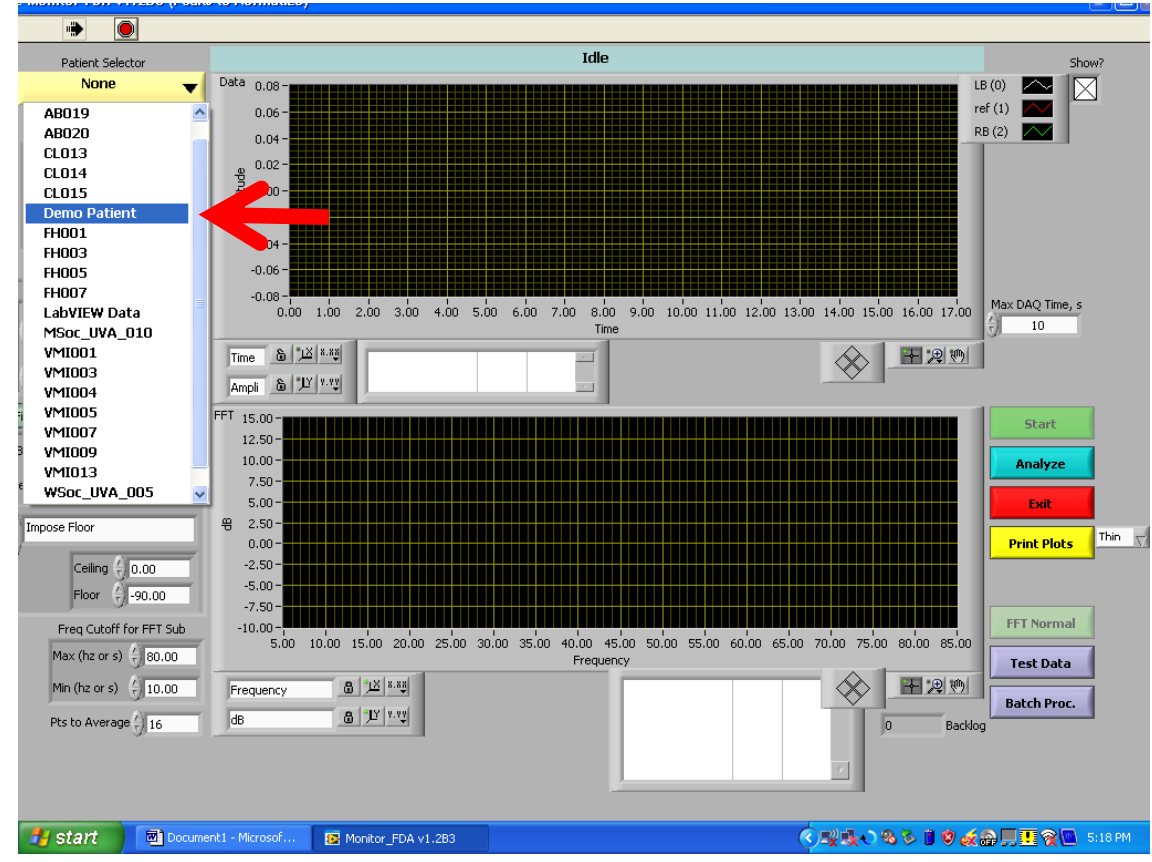

\section{Figure C4.}


Table $\mathrm{C} 10$. Process for obtaining BAM readings.

1. Ensure proper patient file is selected (see table C9).

2. Apply BAM sensors to forehead along lateral border of forehead, just below hairline. (Figure C5)

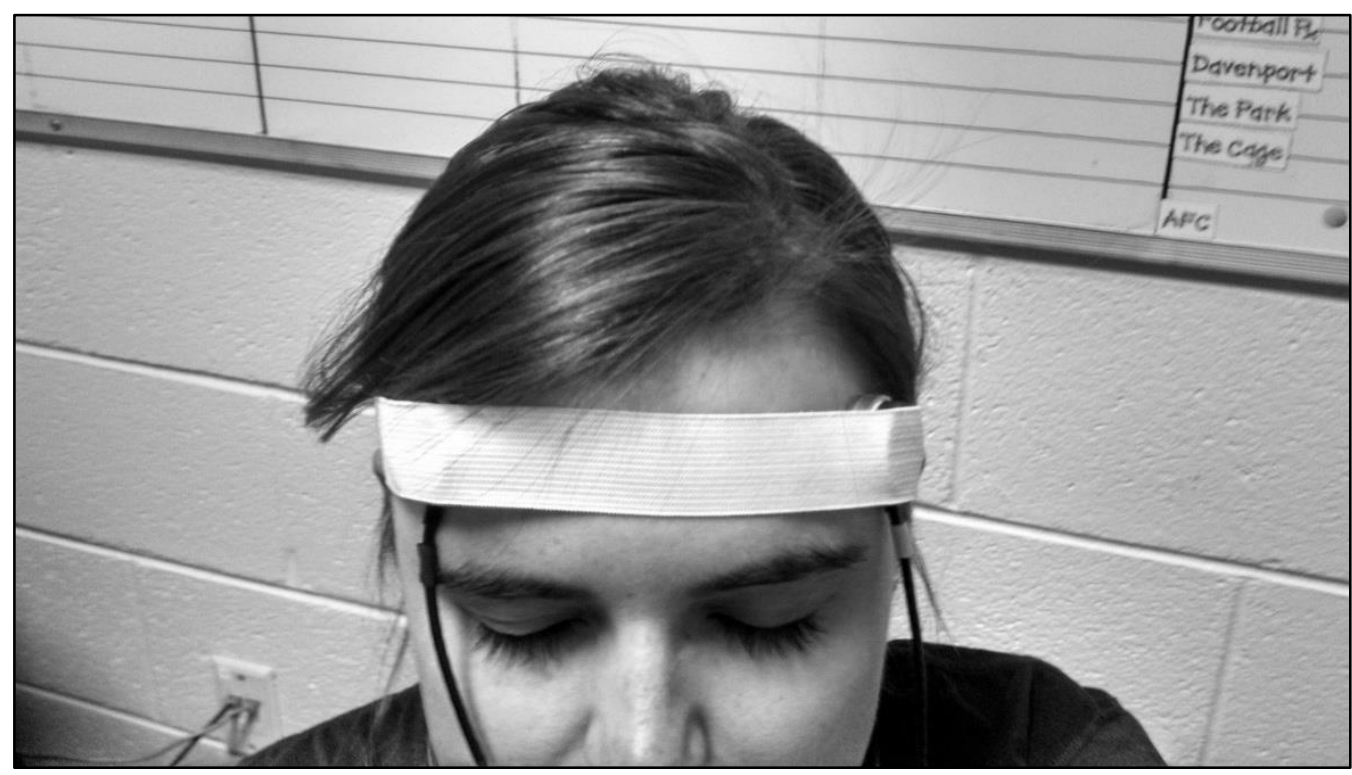

\section{Figure C5.}

3. Apply BAM sensor to left thumb. (Figure C6)

4. Ask subject to close his/her eyes and to remain as still as possible.

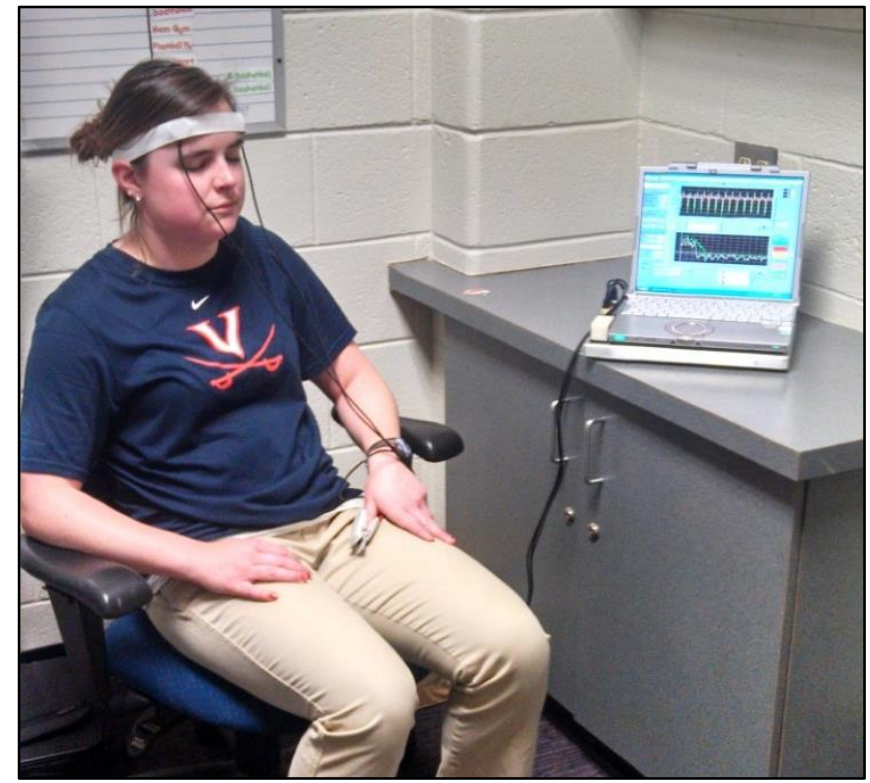

Figure C6. 
5. Run BAM application by clicking green "START" button (Figure C7).

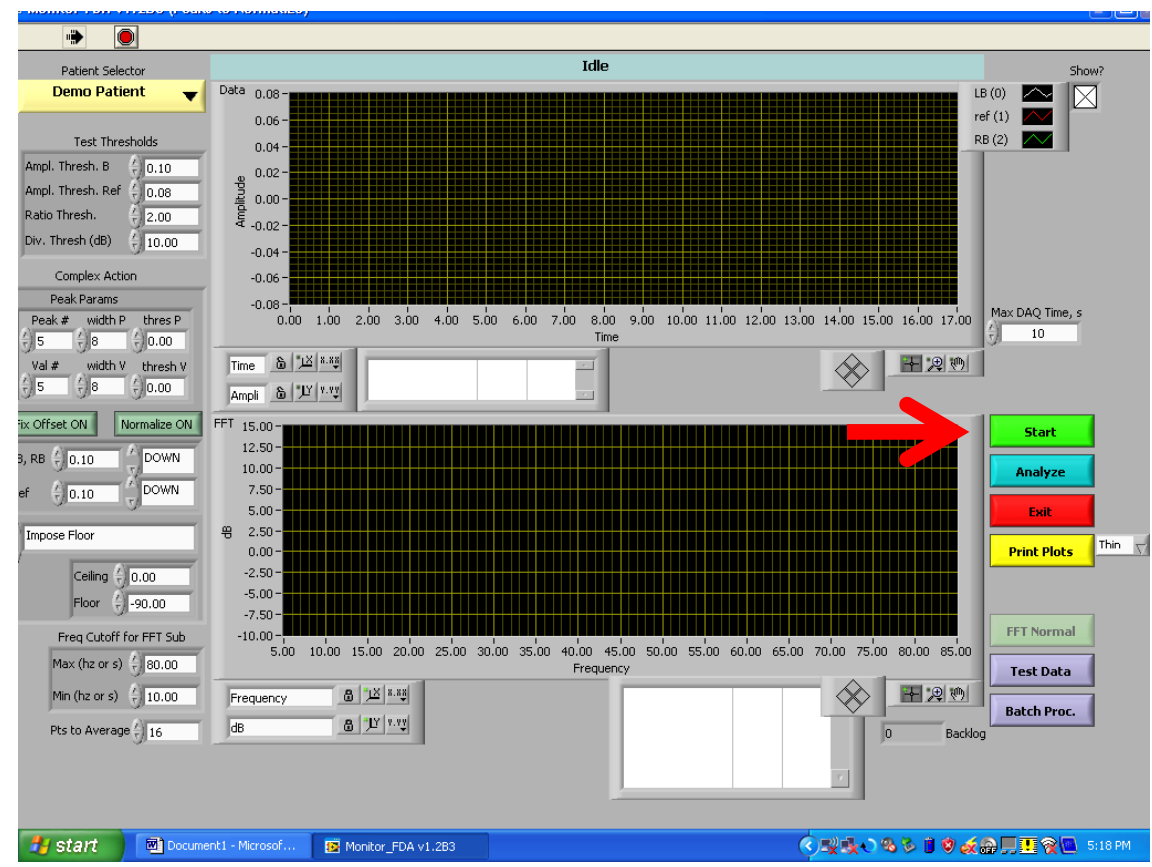

Figure C7.

6. Evaluate BAM and reference signals for adequate amplitude during first 3-5 trials, adjusting sensors as necessary. Also evaluate noise. BAM signal should have two or three clear peaks per systolic pulse cycle. (Figure C8).

a. A box with "analyze" or "reject" data will appear after each BAM reading. Until a satisfactory signal is obtained, click "reject data" (Figure C9)

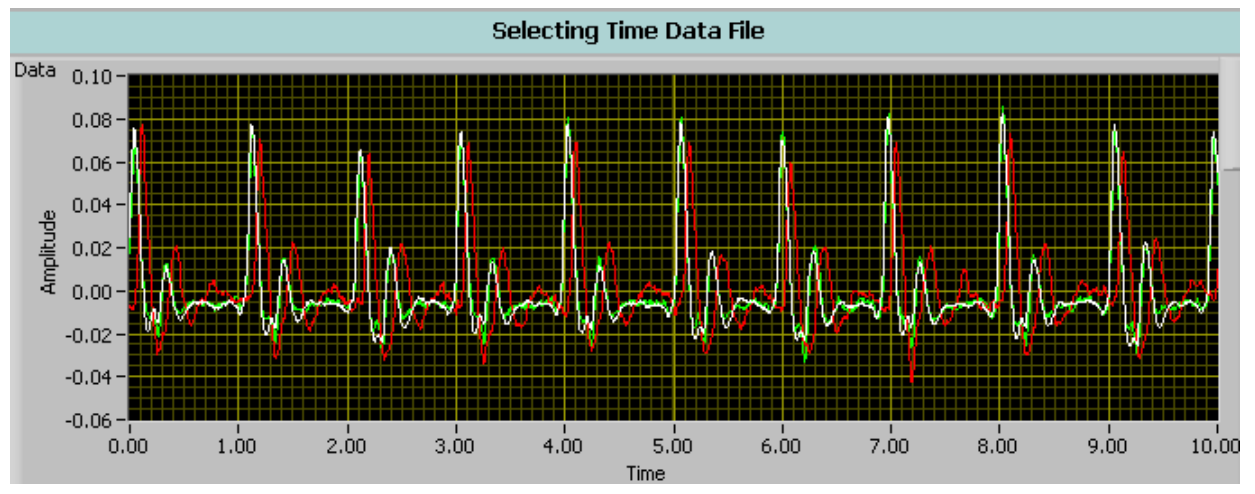

Figure C8. 


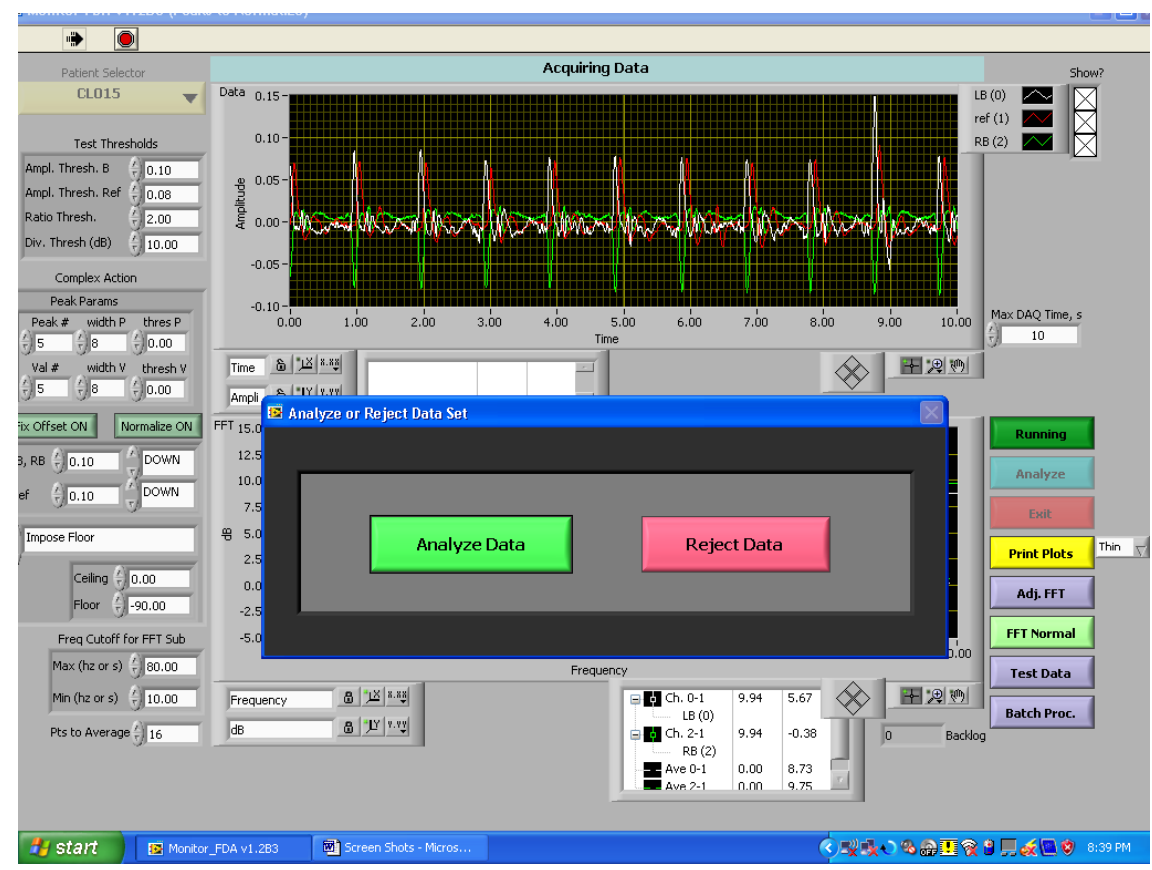

\section{Figure C9.}

7. Once satisfactory signal is obtained, click "START". Once the 10 -second reading has completed, click "analyze data" in the box. This will store the data for that reading and result in a "red", "yellow", or "green" box. If the box is green (Figure C10), click "OK" and then "START" to begin next reading. If the box is yellow or red (Figure C11), click "repeat test" to begin next reading.

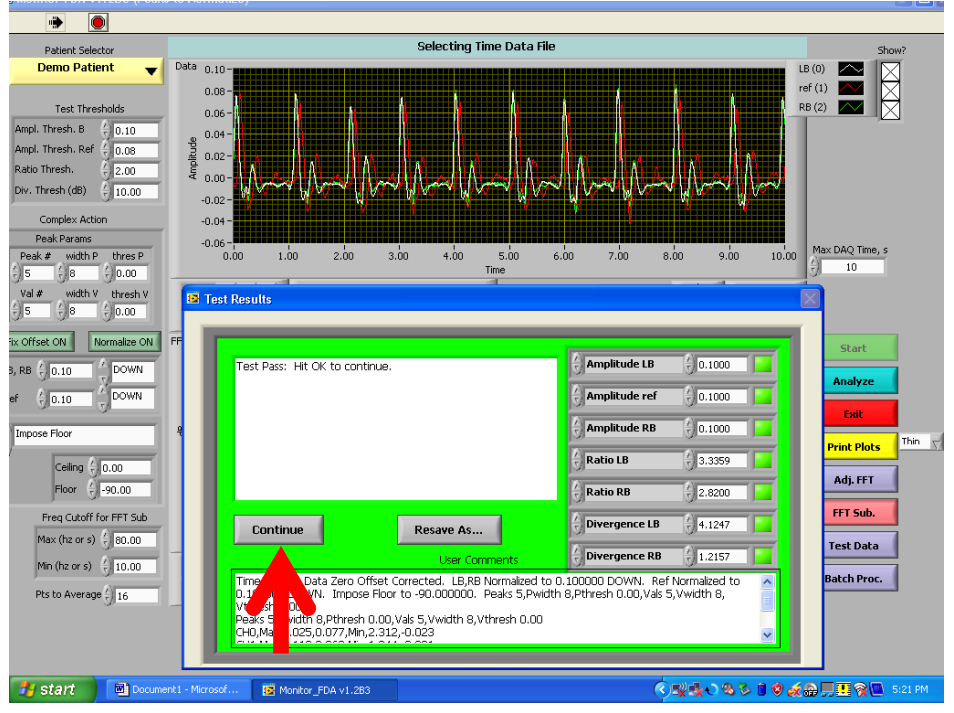

Figure C 10. 


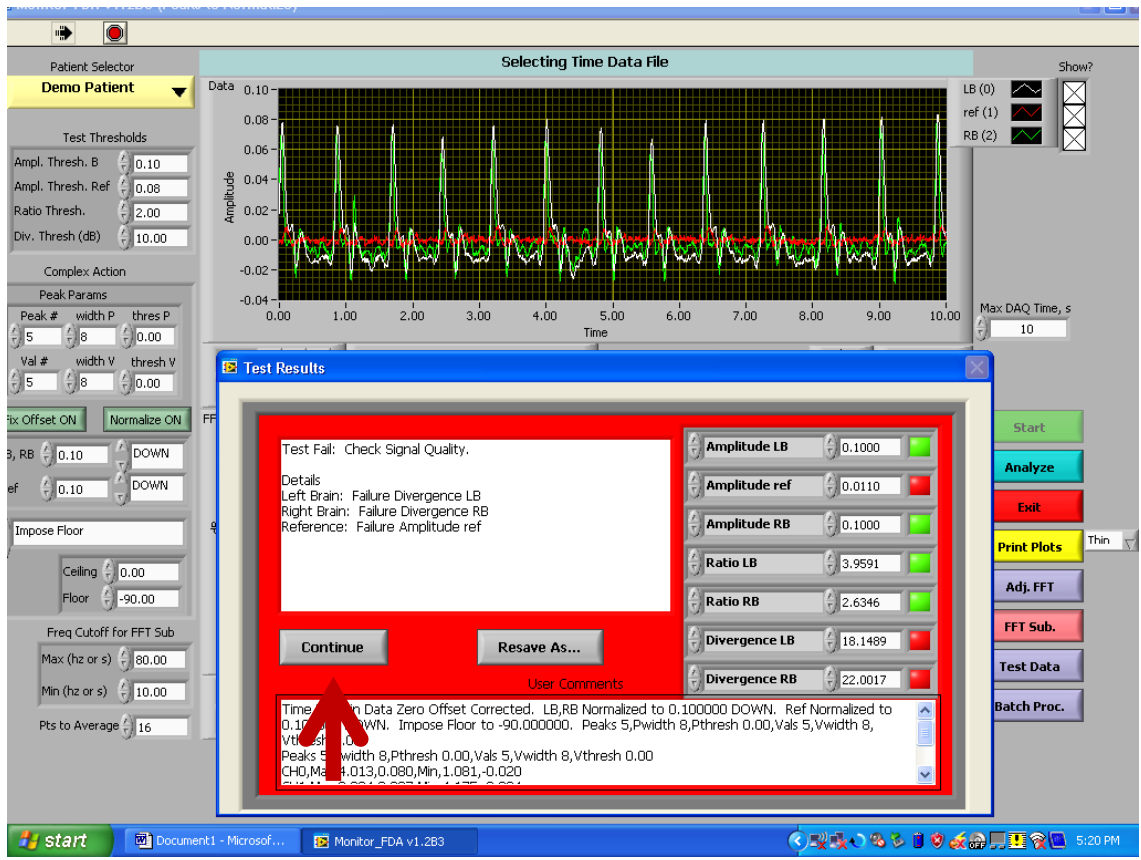

\section{Figure C 11.}

8. 3-5 BAM readings were collected for later processing.

\section{Table C11. SCAT3 Testing Procedures.}

1. Patient fills out demographics and concussion history section

2. Instruct patient to fill out symptom scale. "You should score yourself on the following symptoms, based on how you feel now. A 0 means you do not currently have that symptom and a 6 means you are experiencing that symptom to the worst degree possible".

a. Score symptom scale by first summing total number of reported symptoms (out of 22) then by summing severity of symptoms rated (total possible 132).

3. Perform standardized assessment of concussion.

a. Orientation: ask each question as indicated on the SCAT3 form. Score 1 point for each correct answer and 0 points for incorrect answers. Sum total correct answers for orientation subscore (out of 5).

b. Immediate Memory: Prior to beginning, instruct patient: "I will read a list of 5 words to you. When I am finished, I want you to repeat them back to me, as many as you can remember. They do not have to be in the order that I say them." Read the words at a rate of one per second. Score 1 point for each correct response. Repeat 2 more times. Instruct patient: "I am going to 
repeat the same list of words. Repeat back to me as many as you can remember, including the ones you already said." Sum the scores for all 3 trials to obtain immediate memory subscore (out of 15).

c. Concentration: Prior to beginning, instruction patient: "I will read a list of numbers to you. I want you to repeat them back to me in reverse order. So, if I say '7-1-9' you would repeat '9-1-7'." If correct, go to next string length. If incorrect, read trial 2. If second trial is incorrect, stop testing and score remaining strength lengths as incorrect. The digits should be read at the rate of one per second. Months in reverse is then performed. Instruct patient: "Tell me the months of the year in reverse order. Start with the last month and go backward." Score 1 point for entire sequence correct. Sum correct string repetitions and months backward for concentration subscore (out of 5).

d. Delayed recall: perform this test after completion of the modified BESS test. Instruct patient: "Can you remember any of the words I asked you earlier? List as many as you can remember, in any order". Score 1 point for each correct response to get delayed recall subscore (out of 5 points).

e. Orientation, immediate memory, concentration, and delayed recall are summed to obtain SAC total score.

\section{Perform Modified Balance Error Scoring System.}

a. Double leg stance:

○ Instruct patient: "I am going to test your balance using three 20 -second tests in different stances. Your goal will be to maintain the position as best you can. The first stance is standing with your feet together with your hands on your hips and your eyes closed. You should try to maintain this position as best you can. I will start the timer at the count of 'ready, set, go'."

○ During testing, count errors accumulated by the patient.

b. Single Leg Stance:

○ First determine which foot is the patient's dominant foot by asking which foot they would kick a ball with. Then, have them stand on the opposite (nondominant) foot.

○ Instruct patient: "Now I want you to stand on your right/left foot with your hands on your hips and your eyes closed. Try to maintain this position as best you can. If you stumble, have to put your foot down, take your hands off your hips, or open your eyes, return to the start position and continue balancing. I will start timing at the count of 'ready, set, go'.'

- Again, count errors during testing.

c. Tandem Stance:

○ Instruct patient: "Now I want you to stand with your left/right (nondominant) foot placed directly behind your other foot, with your heel and toe touching. You will, again, place your hands on your hips 
and close your eyes. If you stumble, have to take a step to maintain balance, take your hands off your hips, or open your eyes, return to the start position and continuing balancing. I will start timing on the count of 'ready, set, go'."

○ Count errors during testing

d. Sum errors for all 3 stances at the end of the test. Subtract total errors from 30 to obtain the BESS total score (such that a higher score indicates better balance/fewer errors).

5. Perform the Coordination Examination.

a. Instruct patient in finger-to-nose test: "Now we are going to do a test of your coordination. I want you to pick an arm, either one, and touch your finger to your nose as quickly as you can 5 times. Make sure you extend your elbow fully each time.

Table C12. Administration of the Concussion Resolution Index.

1. After logging into CRI website using UVA customer code, username, and password, select "Tests" to choose a test taker profile (Figure C12).

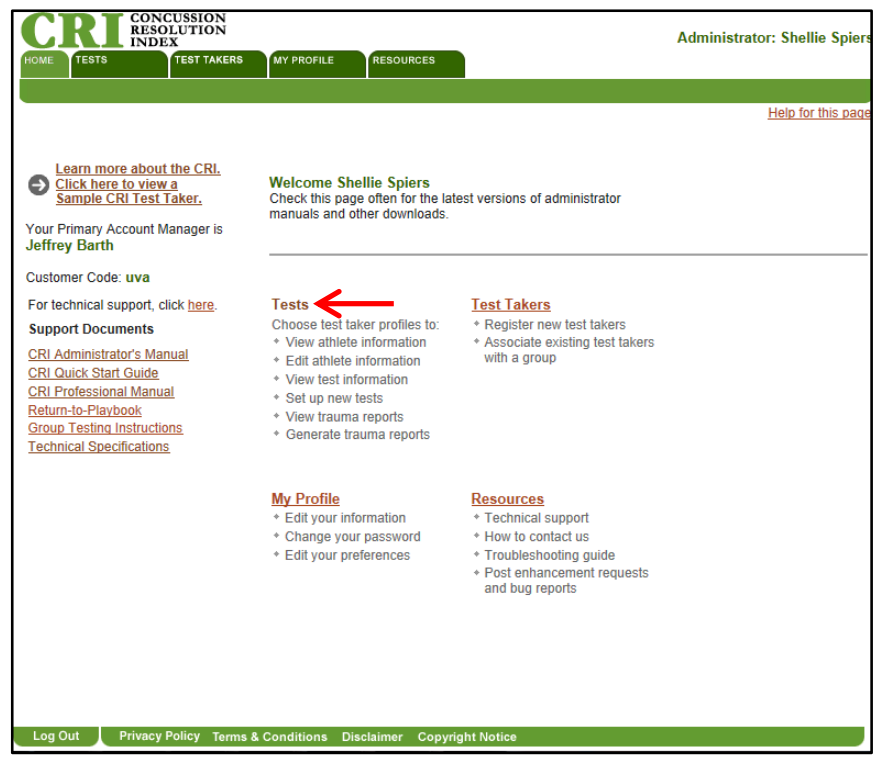

Figure C12. 
2. Using the last name of the test taker, search the UVA database. Then select appropriate test taker profile (Figure C13).

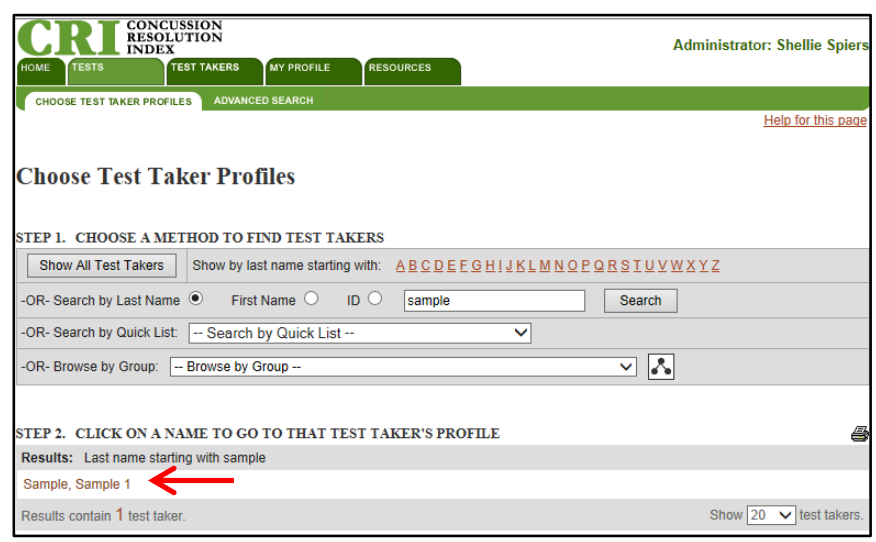

\section{Figure C13.}

3. To set up a baseline test, click "set up new baseline test" at bottom of test taker profile page (Figure C14).

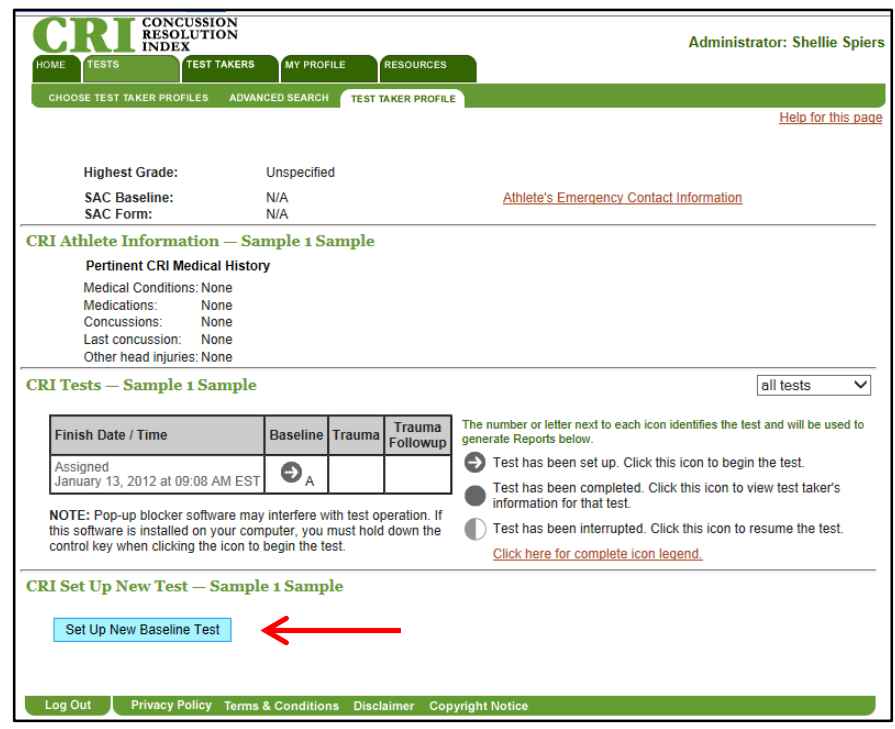

\section{Figure C14.}

4. A new test will have been added to the "CRI Tests" section of the test taker profile. To begin test, click the icon in the "baseline" column (Figure C15). 


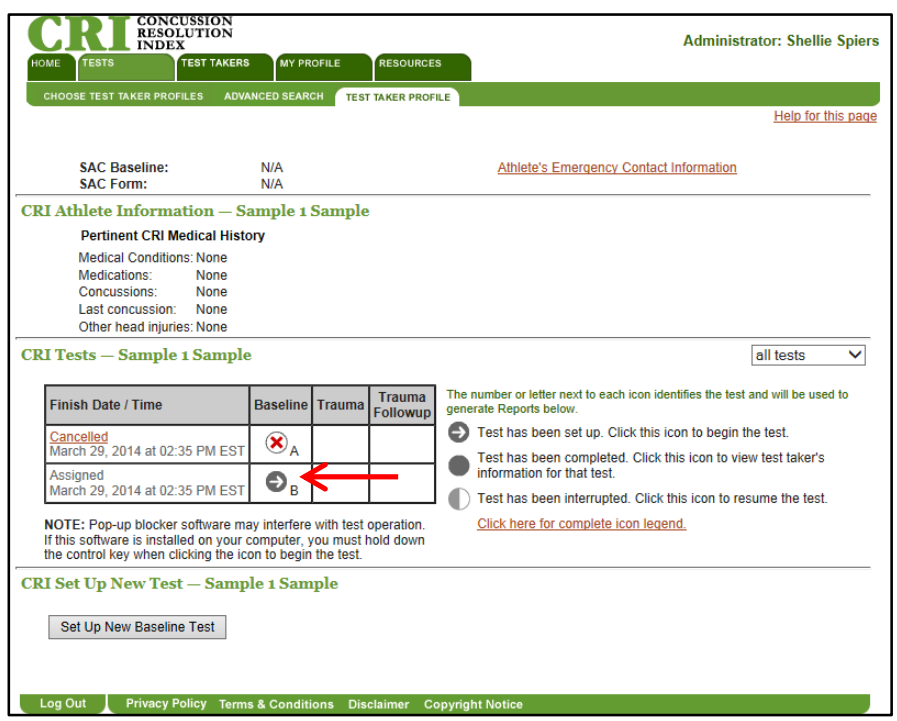

\section{Figure C15}

5. A window containing instructions for administrators will open. All other programs should be closed on the desktop and any audible or automatic features that may distract the test taker should be turned off. Once appropriate environment was obtained, click "next" (Figure C16).

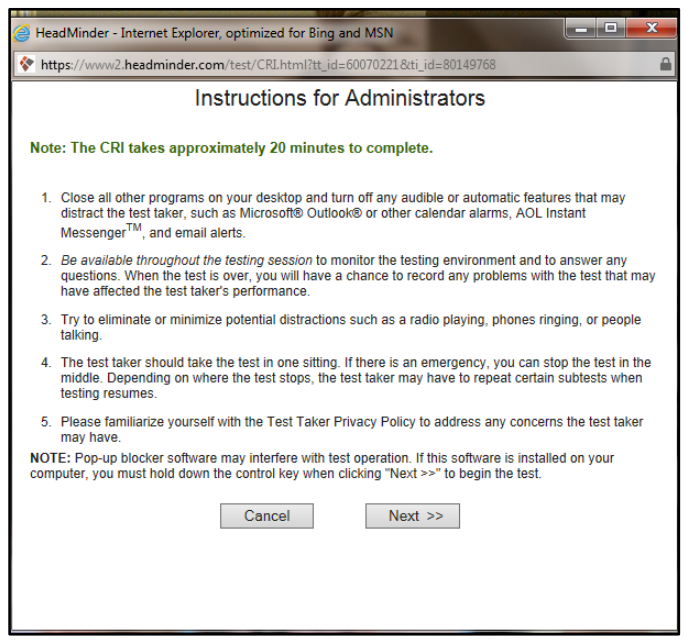

Figure C16.

6. Leave athlete to complete test. Baseline tests will first ask questions about demographics, primary contact information, prior head injury history, other general medical history, hand dominance, language preference, history of learning disorders, migraines, and psychological disorders, and medications currently taken. Post-injury examinations will include questions regarding post-injury symptoms, current symptoms, and injury mechanism. 
7. Upon completion of the test, the test administrator will be asked to log back into the CRI database to validate the test (Figures C17 and C18).

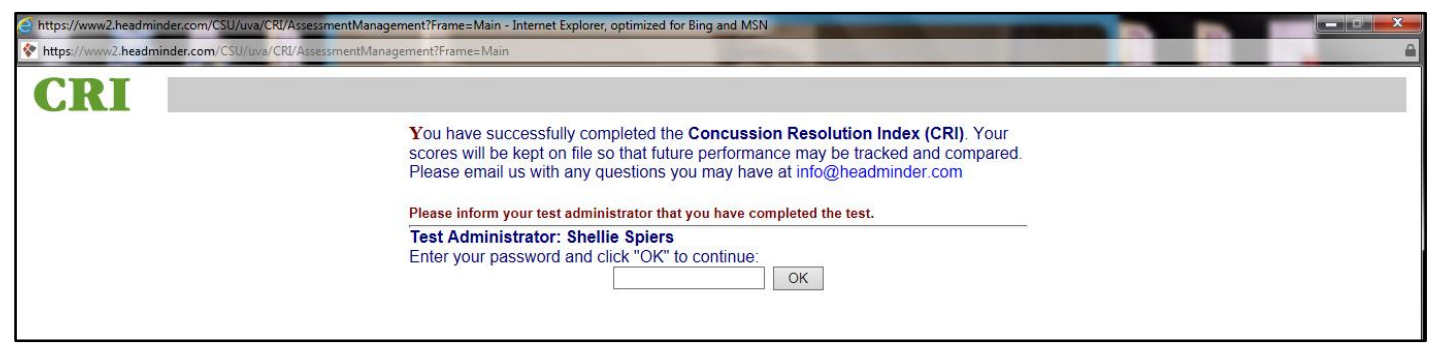

Figure C17.

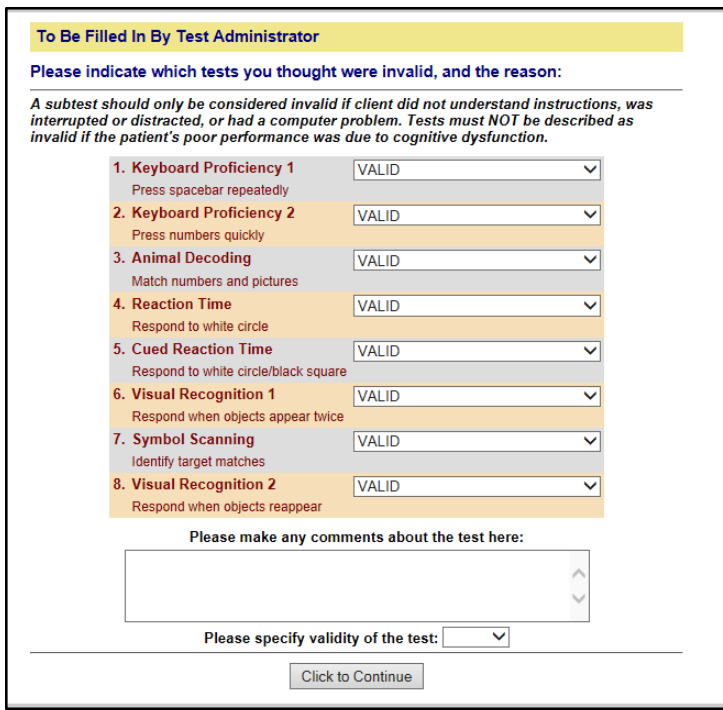

Figure C18.

Table C13. Data processing procedures for BAM data.

1. Convert all BAM time domain (.STP) files to Microsoft Excel 97-03 Format (.XLS). BAM files can be copied from BAM computer by accessing the "My Documents Folder" on the desktop (Figure C19). 


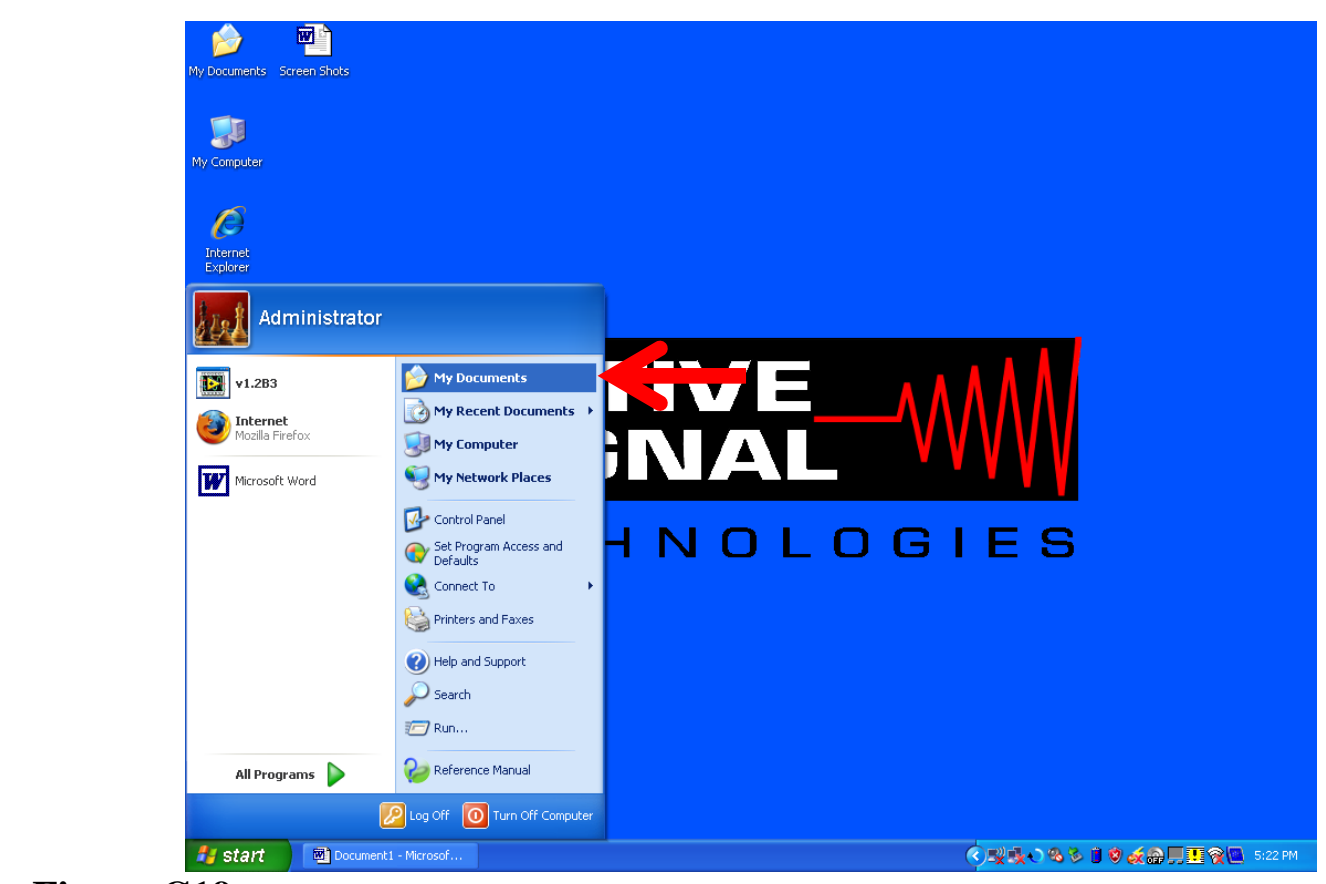

Figure C19

a. Locate patient file name (as you indicated during BAM testing). Double click to open (Figure C20).

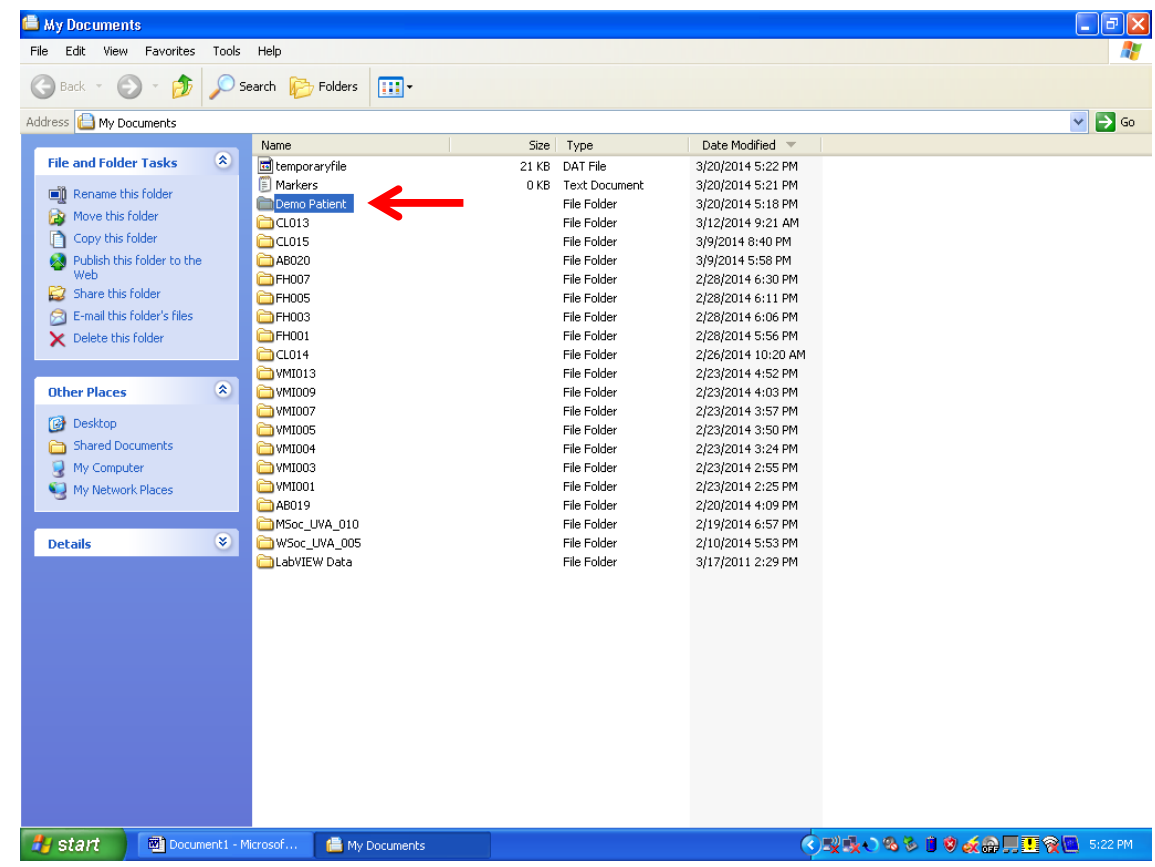

Figure C20. 
b. Files with the "STP" extension are used for Matlab processing (Figure C21). Copy these files and convert by opening in Microsoft Excel. Click "save as" and change file format to "Excel 97-2003 Workbook".

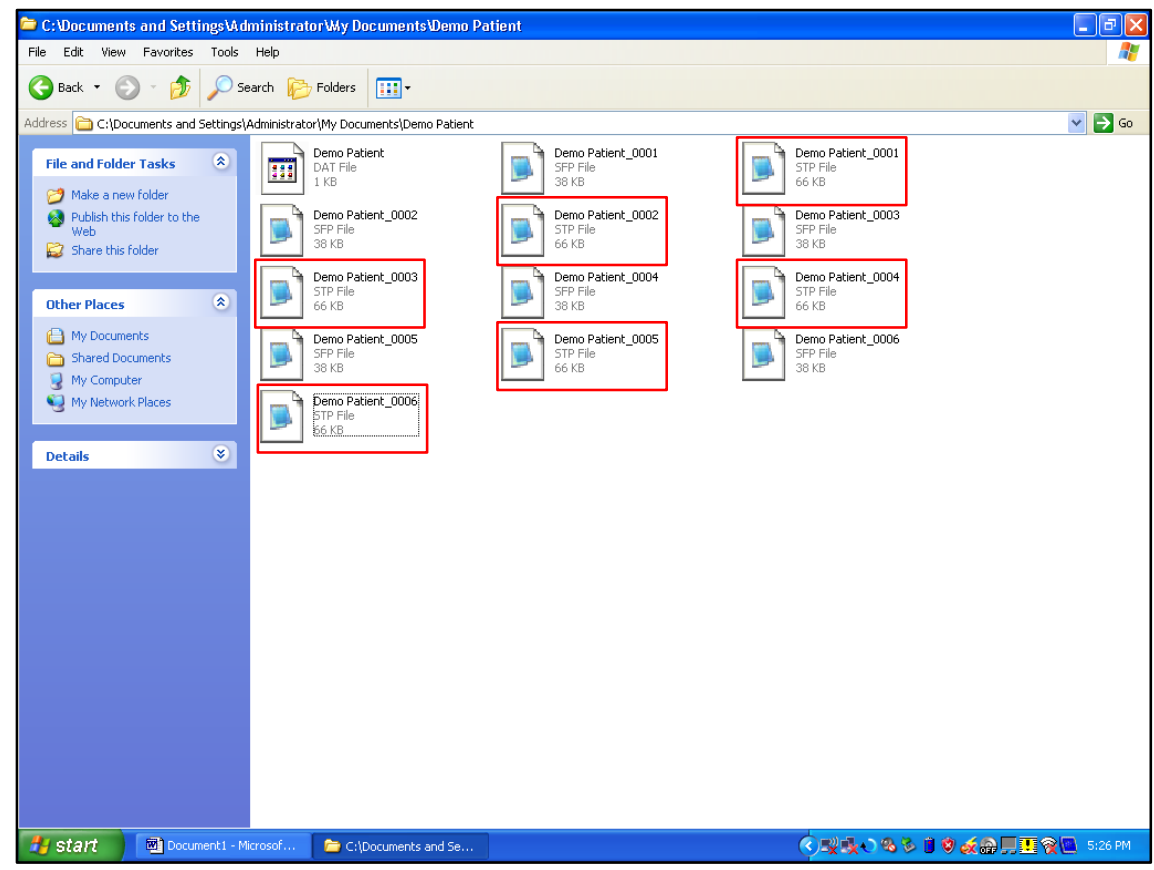

\section{Figure C21.}

2. In Matlab "current folder" window, browse for folder containing BAM scripts. Right click and select "add to path" $\rightarrow$ "selected folders and subfolders" (Figure C22).

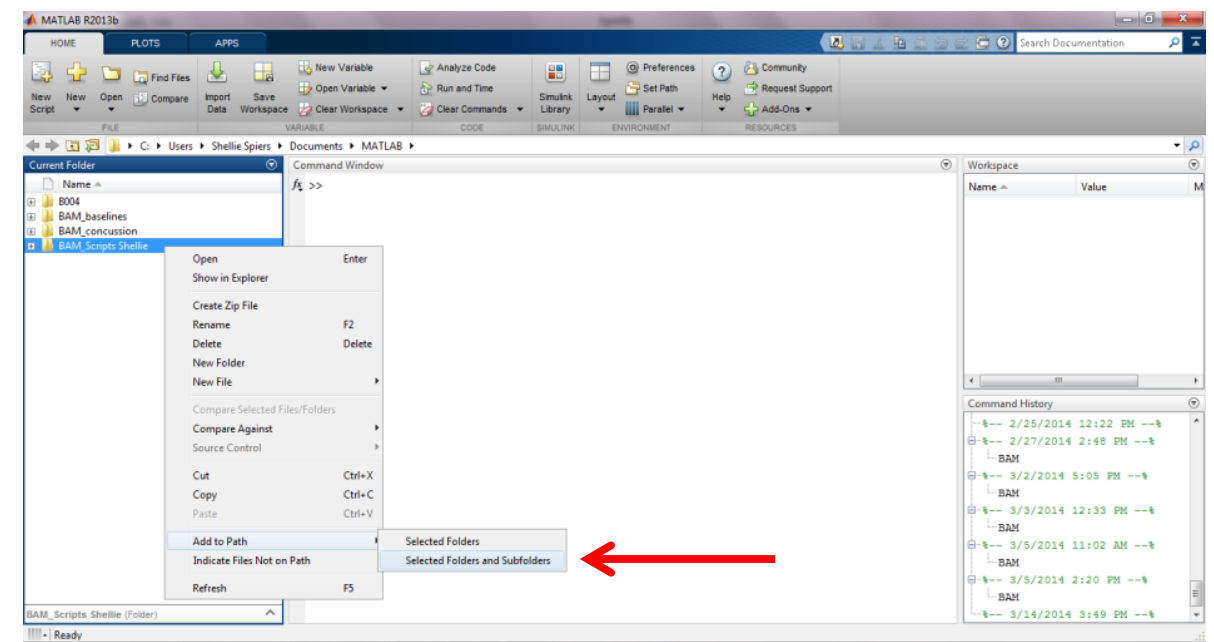

Figure C22. 
3. Then open folder containing BAM scripts. Double click program named "BAM.m" (Figure C23). This will open a second window containing the script for BAM signal processing.

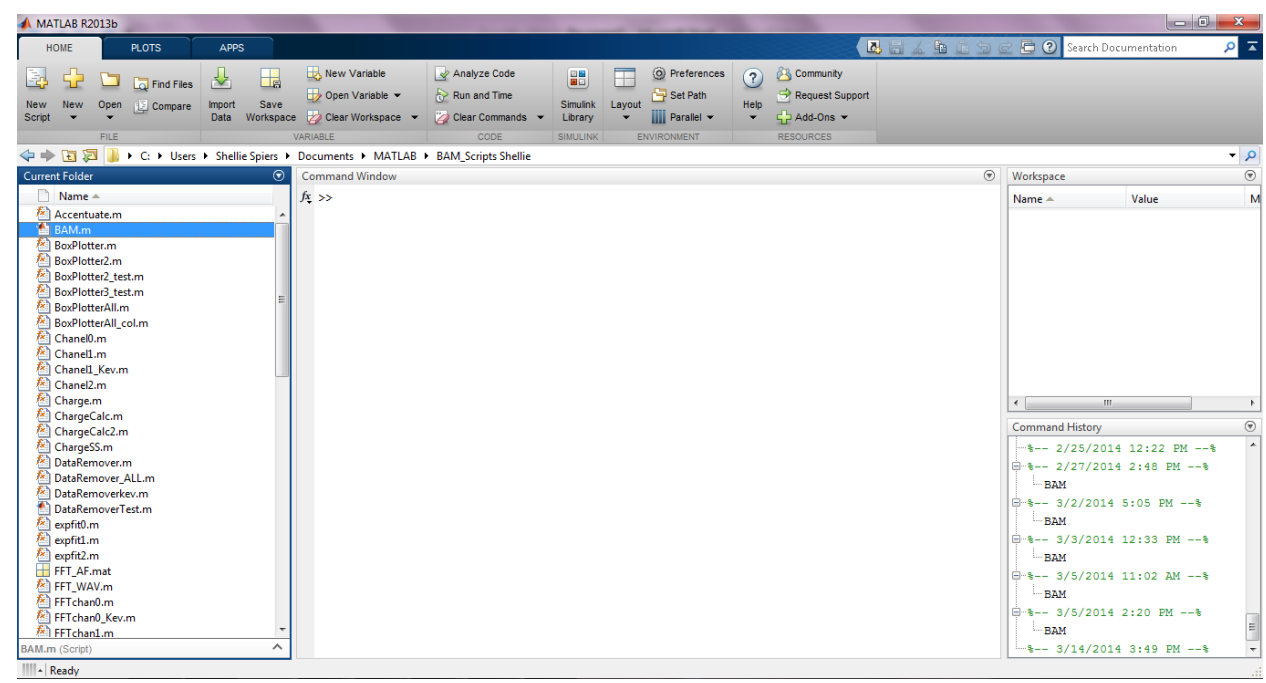

\section{Figure C23.}

4. In new window containing BAM script, scroll down to "Step Two". Within the quotations, designate a file name for new output to be written to (Figure C24).

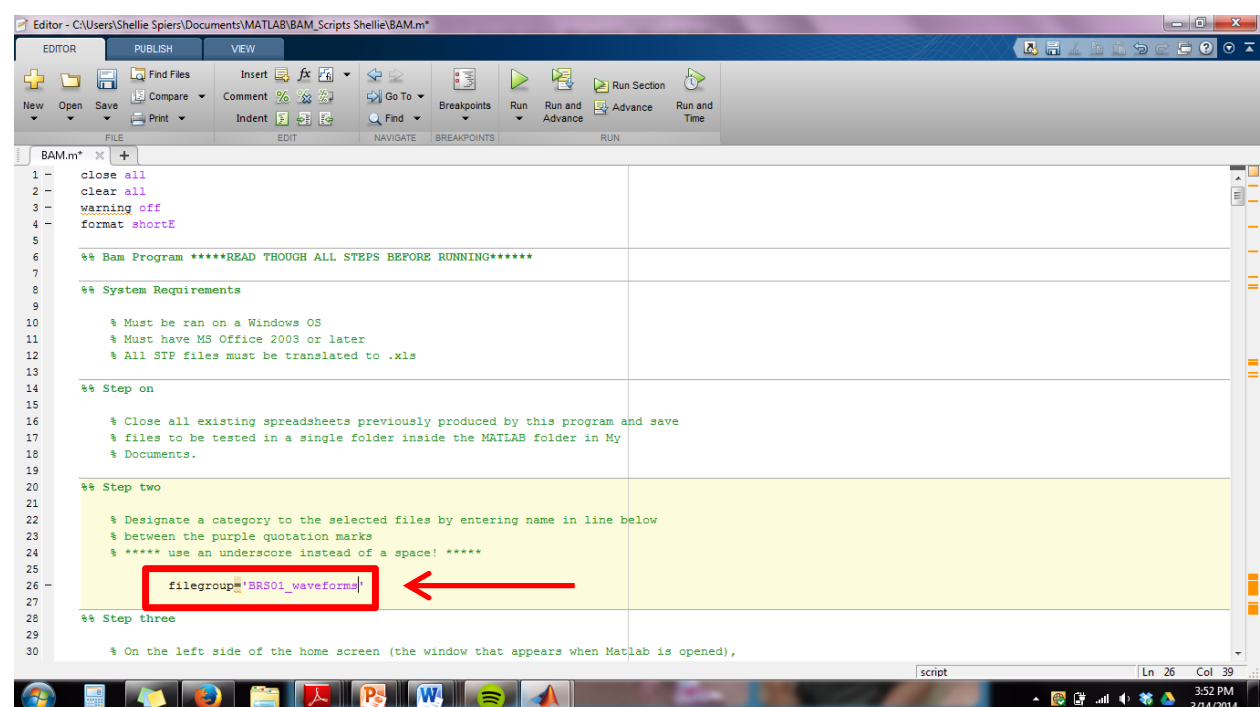

Figure C24. 
5. Then scroll to "Step Five" to indicate method of identifying files to analyze. Select "all" by putting a " 1 " within the brackets to run all files within a folder. Select "files" by putting a " 1 " within the brackets to run a range of files based on file name. Select "index" by putting a " 1 " within the brackets to run a range of files based on the order within the selected folder (Figure C25).

1. If you select "all", no additional information is needed.

2. If you select to run files by file name, you will need to input specific file range based on file name within the appropriate window.

3. If you select to run files by index, you will need to specify the range of files within the selected folder within the appropriate window.

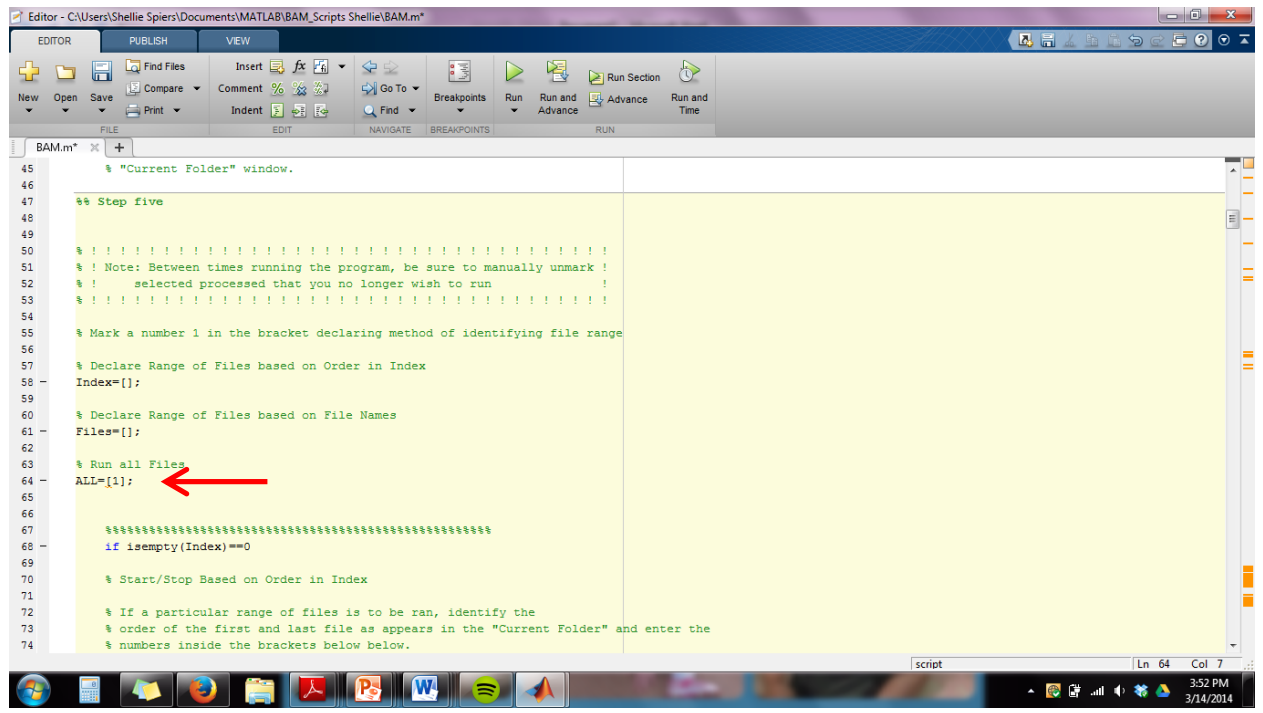

\section{Figure C25.}

6. Scroll down to "Step Six" to indicate which processes to run. Typically, "Waveform_Analysis", "Raw_Data_Plot", and "Waveform_Plot" were selected.

1. To indicate that a processes is selected, put a "1" within the brackets next to each (Figure C26). 


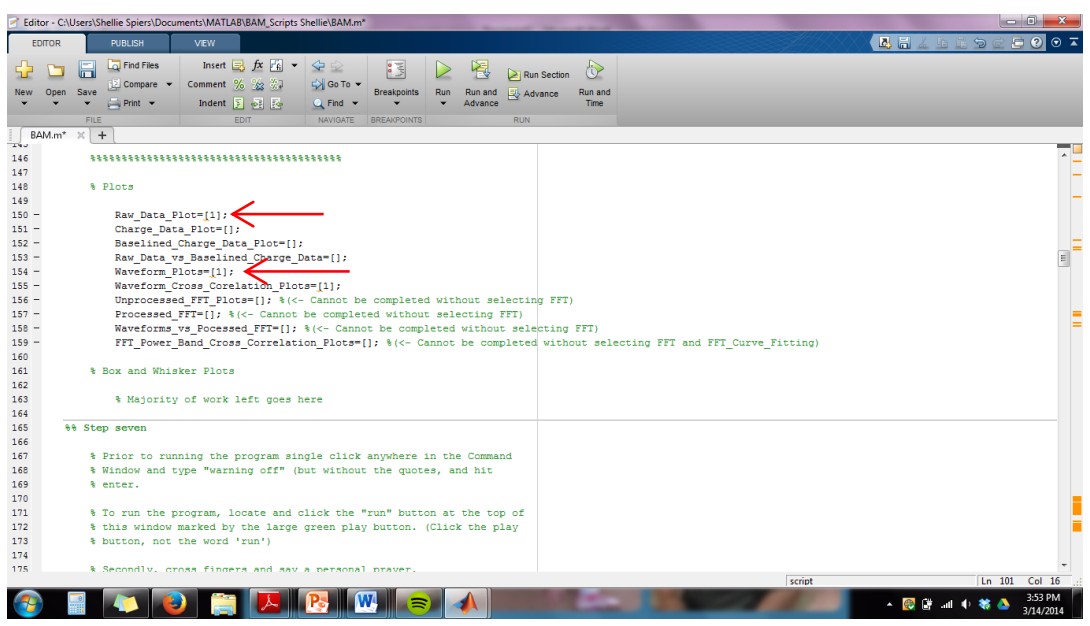

\section{Figure C26.}

7. Within the main Matlab window, browse for the files you wish to process. Do not open the window. Select the folder containing the appropriate BAM files and then click "select folder" (Figure C27). This should result in the "current folder" containing all files you wish you select. Note: only files within the folder will run. If the folder contains other folders, the files will not be run within those folders.

8. Once the appropriate folder has been selected, go back to BAM script window and click "run" (Figure C28).

9. Once the program has finished running files, the main Matlab window will display a message reading "Program Terminated".

10. BAM files containing processed data can be found in the "BAM_data" folder that is created within your "My documents" folder (on Windows OS).

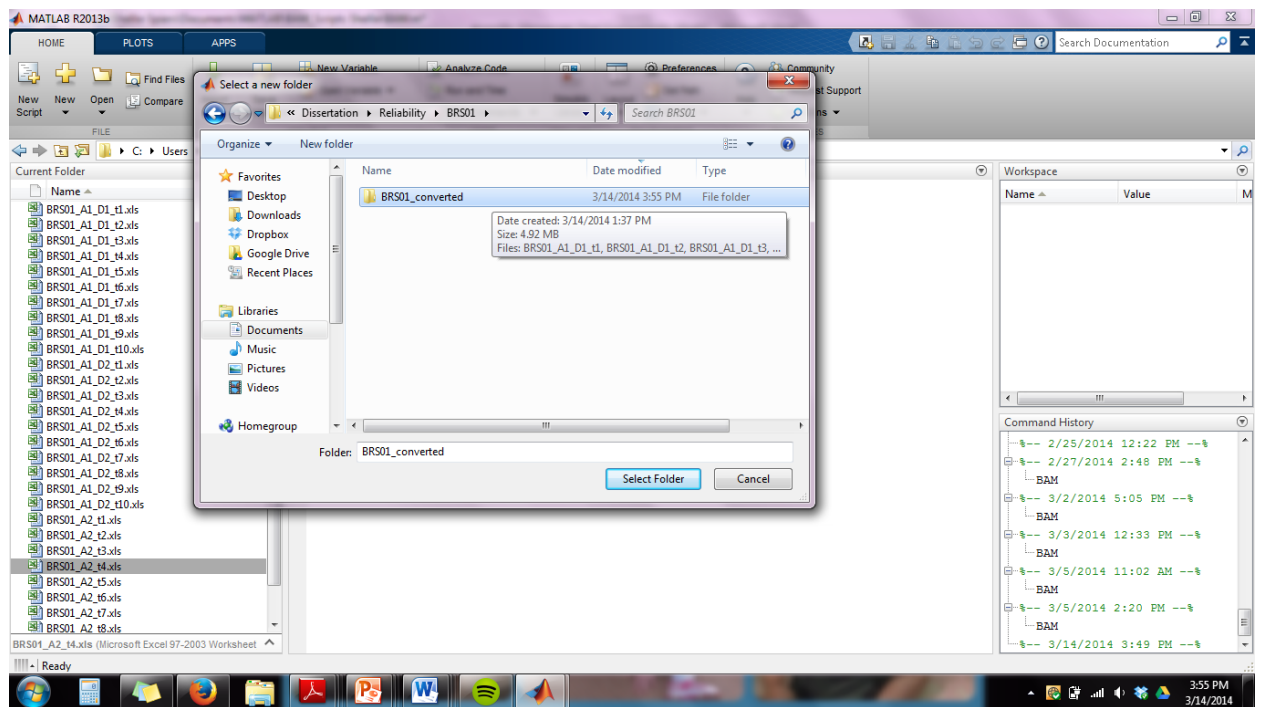

Figure C27. 


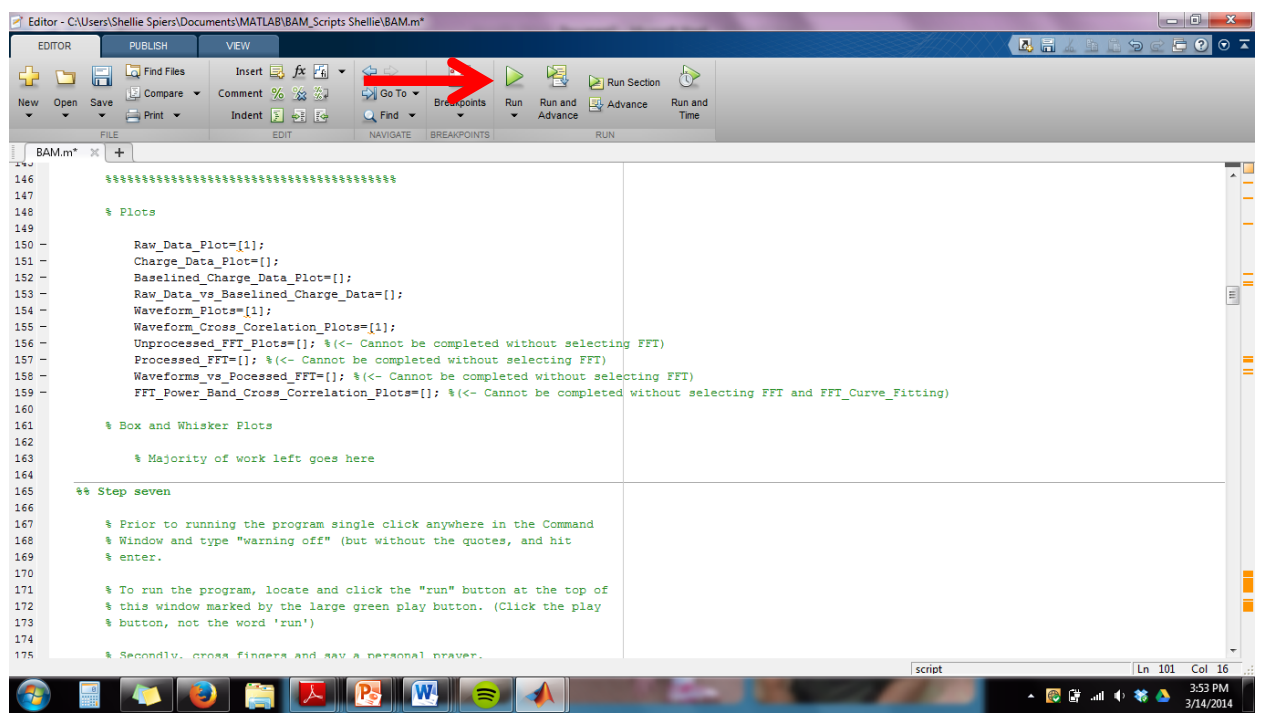

\section{Figure C28.}

\section{Table C14. Extraction of CRI indices.}

1. Log in to CRI database using UVA customer code, user name and password.

2. Select appropriate test taker profile by clicking "tests" menu.

3. Use last name of test taker to search for his/her profile.

4. Once in test taker profile, click on the most recent (or desired) baseline or post injury examination icon within the appropriate column of the "CRI Tests" table (Figure C29).

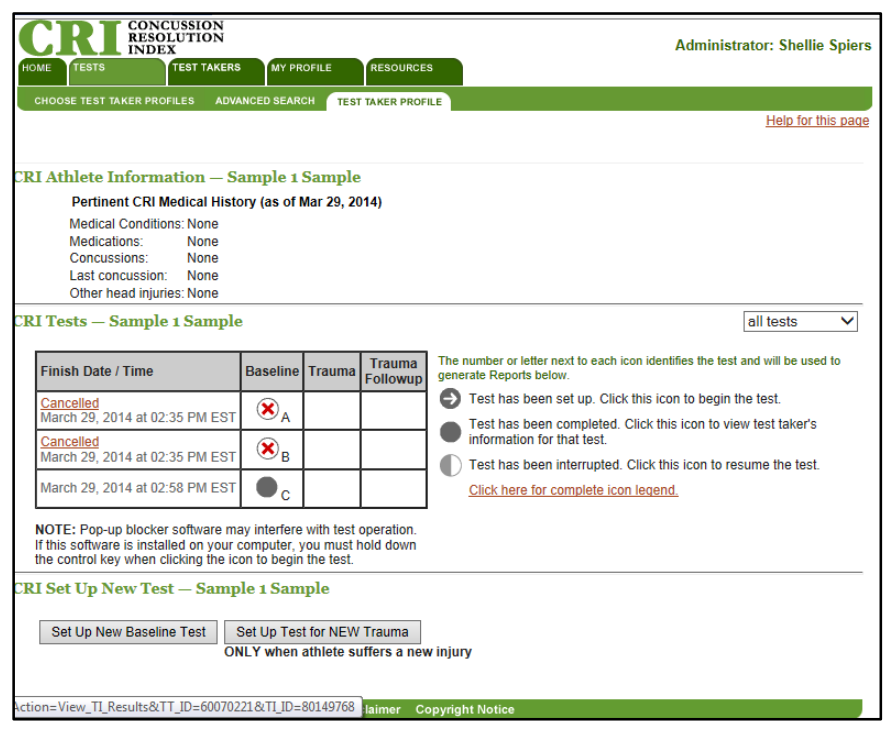

Figure C29. 
5. This will open a window containing a break-down of patient demographics, concussion history, medical history, medication, symptoms, and scores for each subtest of the CRI.

6. Scroll to the bottom of the page to find the "Summary Data".

7. Data extracted is under "raw scores" (Figure C30).

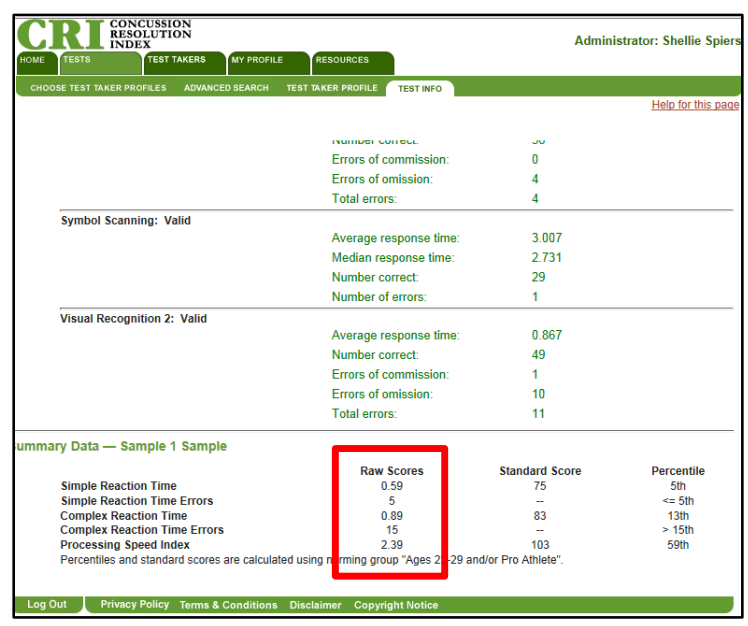

Figure C30. 


\section{APPENDIX D}

\section{Additional Results}

\section{Manuscript 1}

$\underline{\text { Table D1. Descriptive statistics for subject demographics and gender frequency }}$

\section{Descriptive Statistics}

\begin{tabular}{|l|l|l|l|l|l|}
\hline & $\mathrm{N}$ & Minimum & Maximum & Mean & Std. Deviation \\
\hline height & 19 & 160.02 & 193.04 & 172.9874 & 8.41975 \\
weight & 19 & 52.16 & 99.79 & 74.6039 & 13.81647 \\
Age & 19 & 18.00 & 31.00 & 24.1579 & 3.96181 \\
Valid N (listwise) & 19 & & & & \\
\hline
\end{tabular}

gender

\begin{tabular}{|ll|l|l|l|l|l|}
\hline & & Frequency & Percent & Valid Percent & Cumulative Percent \\
\hline \multirow{4}{*}{ Valid } & .00 & 8 & 42.1 & 42.1 & 42.1 \\
& 1.00 & 11 & 57.9 & 57.9 & 100.0 \\
& Total & 19 & 100.0 & 100.0 & \\
\hline
\end{tabular}

Table D2. Descriptive statistics for left and right systolic interval, steepest rise gradient, peak interval, and augmentation index for Day 1 and Day 2 measurements.

Descriptive Statistics

\begin{tabular}{|c|c|c|c|c|c|c|c|c|}
\hline & \multirow[t]{2}{*}{$\mathrm{N}$} & \multirow[t]{2}{*}{ Mean } & \multirow{2}{*}{$\begin{array}{l}\text { Std. } \\
\text { Deviation }\end{array}$} & \multirow{2}{*}{\multicolumn{2}{|c|}{ Minimum Maximum }} & \multicolumn{3}{|c|}{ Percentiles } \\
\hline & & & & & & $25^{\text {th }}$ & $\begin{array}{l}50 \text { th } \\
\text { (Median) }\end{array}$ & 75 th \\
\hline LSysInt_D1 & 19 & .1407 & .04789 & .09 & .26 & .1115 & .1240 & .1510 \\
\hline LSRG_D1 & 19 & 3.1363 & 1.59978 & 1.61 & 7.63 & 1.7971 & 2.8160 & 3.5946 \\
\hline LPI_D1 & 19 & .1346 & .04734 & .09 & .27 & .1052 & .1219 & .1469 \\
\hline LAIX_D1 & 19 & 10.0165 & 15.80287 & .00 & 58.18 & .0000 & 4.4737 & 11.2485 \\
\hline RSystInt_D1 & 19 & .1591 & .07004 & .05 & .27 & 1031 & .1281 & .2344 \\
\hline RSRG_D1 & 19 & 3.3227 & 1.52088 & 1.04 & 5.95 & 1.8187 & 3.3322 & 4.6877 \\
\hline RPI_D1 & 19 & .1391 & .06071 & .06 & .27 & .1052 & .1177 & .1469 \\
\hline RAIX_D1 & 19 & 12.8067 & 9.64850 & .00 & 33.67 & 7.0862 & 11.0363 & 17.1670 \\
\hline LSysInt_D2 & 19 & .1620 & .06721 & .07 & .27 & .1052 & .1344 & .2344 \\
\hline LSRG_D2 & 19 & 3.0148 & 1.21818 & 1.61 & 5.92 & 2.0797 & 2.4728 & 3.6644 \\
\hline LPI_D2 & 19 & .1480 & .04403 & .09 & .26 & .1240 & .1344 & .1531 \\
\hline LAIX_D2 & 19 & | 17.1497 & 14.87332 & .00 & 51.06 & 6.6868 & 14.9813 & 23.2518 \\
\hline RSystInt_D2 & 19 & .1697 & .06421 & .08 & .27 & .1073 & .1677 & .2427 \\
\hline RSRG_D2 & 19 & 3.2785 & 1.74842 & .92 & 7.66 & 2.2262 & 2.8399 & 3.7525 \\
\hline RPI_D2 & 19 & .1457 & .04884 & .08 & .27 & .1094 & .1302 & .1719 \\
\hline RAIX_D2 & 19 & 12.0351 & 9.36020 & .68 & 40.19 & 4.7853 & 12.2114 & 14.8076 \\
\hline
\end{tabular}


Table D3. Wilcoxon Signed-Rank Test comparing Day 1 and Day 2 measures of left systolic interval, steepest rise gradient, peak interval, and augmentation index.

\section{Ranks}

\begin{tabular}{|c|c|c|c|c|}
\hline & & $\mathrm{N}$ & Mean Rank & Sum of Ranks \\
\hline & Negative Ranks & $10^{\mathrm{a}}$ & 7.20 & 72.00 \\
\hline LSYSINT_D2 - & Positive Ranks & $8^{\mathrm{b}}$ & 12.38 & 99.00 \\
\hline LSYSINT_D1 & Ties & $1^{\mathrm{c}}$ & & \\
\hline & Total & 19 & & \\
\hline & Negative Ranks & $12^{\mathrm{d}}$ & 9.17 & 110.00 \\
\hline LSRG D2 - LSRG D1 & Positive Ranks & $7^{\mathrm{e}}$ & 11.43 & 80.00 \\
\hline LSNU_DL-LSNU_DI & Ties & $0^{\mathrm{f}}$ & & \\
\hline & Total & 19 & & \\
\hline & Negative Ranks & $8^{g}$ & 5.75 & 46.00 \\
\hline I PKINT D2 - LPKINT D1 & Positive Ranks & $11^{\mathrm{h}}$ & 13.09 & 144.00 \\
\hline LFNIN1_DL-LFNINI_DI & Ties & $0^{i}$ & & \\
\hline & Total & 19 & & \\
\hline & Negative Ranks & $4^{j}$ & 10.50 & 42.00 \\
\hline LAUGIND_D2 - & Positive Ranks & $14^{\mathrm{k}}$ & 9.21 & 129.00 \\
\hline LAUGIND_D1 & Ties & $1^{1}$ & & \\
\hline & Total & 19 & & \\
\hline & Negative Ranks & $8^{m}$ & 7.75 & 62.00 \\
\hline RSYSINT_D2 - & Positive Ranks & $9^{n}$ & 10.11 & 91.00 \\
\hline RSYSINT_D1 & Ties & $2^{\circ}$ & & \\
\hline & Total & 19 & & \\
\hline & Negative Ranks & $9^{p}$ & 10.67 & 96.00 \\
\hline RSRG D2 - RSRG D1 & Positive Ranks & $10^{\mathrm{q}}$ & 9.40 & 94.00 \\
\hline КМКU_D & Ties & $0^{\mathrm{r}}$ & & \\
\hline & Total & 19 & & \\
\hline & Negative Ranks & $8^{s}$ & 10.19 & 81.50 \\
\hline RPKINT D2 - RPKINT D1 & Positive Ranks & $11^{\mathrm{t}}$ & 9.86 & 108.50 \\
\hline RPKINI_DL-KPKINI_DI & Ties & $0^{\mathrm{u}}$ & & \\
\hline & Total & 19 & & \\
\hline & Negative Ranks & $11^{\mathrm{v}}$ & 10.36 & 114.00 \\
\hline RAUGIND_D2 - & Positive Ranks & $8^{w}$ & 9.50 & 76.00 \\
\hline RAUGIND_D1 & Ties & $0^{\mathrm{x}}$ & & \\
\hline & Total & 19 & & \\
\hline
\end{tabular}

\begin{tabular}{|l|l|l|l|l|}
\hline & LSYSINT_D2 - & LSRG_D2 - & LPKINT_D2 - & LAUGIND_D2 - \\
& LSYSINT_D1 & LSRG_D1 & LPKINT_D1 & LAUGIND_D1 \\
\hline$Z$ & $.588^{\mathrm{b}}$ & $-.604^{\mathrm{c}}$ & $-1.972^{\mathrm{b}}$ & $-1.894^{\mathrm{b}}$ \\
$\begin{array}{l}\text { Asymp. Sig. } \\
\text { (2-tailed) }\end{array}$ & .556 & .546 & .049 & .058 \\
\hline
\end{tabular}




\begin{tabular}{|l|l|l|l|l|}
\hline & $\begin{array}{l}\text { RSYSINT_D2 - } \\
\text { RSYSINT_D1 }\end{array}$ & $\begin{array}{l}\text { RSRG_D2 - } \\
\text { RSRG_D1 }\end{array}$ & $\begin{array}{l}\text { RPKINT_D2 - } \\
\text { RPKINT_D1 }\end{array}$ & $\begin{array}{l}\text { RAUGIND_D2 - } \\
\text { RAUGIND_D1 }\end{array}$ \\
\hline Z & $-.687^{\mathrm{b}}$ & $-.040^{\mathrm{c}}$ & $-.543^{\mathrm{b}}$ & $-.765^{\mathrm{c}}$ \\
Asymp.Sig. & .492 & .968 & .587 & .445 \\
\hline (2-tailed) & -68 & & \\
\hline
\end{tabular}

$\underline{\text { Table D4. Intraclass Correlation Coefficient for Day } 1 \text { and Day } 2 \text { left systolic interval. }}$

Intraclass Correlation Coefficient

\begin{tabular}{|c|c|c|c|c|c|c|c|}
\hline & \multirow{2}{*}{$\begin{array}{l}\text { Intraclass } \\
\text { Correlation }^{\mathrm{b}}\end{array}$} & \multicolumn{6}{|c|}{ 95\% Confidence Interval } \\
\hline & & $\begin{array}{l}\text { Lower } \\
\text { Bound }\end{array}$ & $\begin{array}{l}\text { Upper } \\
\text { Bound }\end{array}$ & Value & df1 & $\mathrm{df2}$ & Sig \\
\hline Single Measures & $-.012^{\mathrm{a}}$ & -.453 & .434 & 976 & 18 & 18 & .520 \\
\hline $\begin{array}{l}\text { Average } \\
\text { Measures }\end{array}$ & -.024 & -1.659 & .605 & 976 & 18 & 18 & .520 \\
\hline
\end{tabular}

$\underline{\text { Table D5. Intraclass Correlation Coefficient for Day } 1 \text { and Day } 2 \text { left steepest rise gradient. }}$

Intraclass Correlation Coefficient

\begin{tabular}{|c|c|c|c|c|c|c|c|}
\hline & \multirow{2}{*}{$\begin{array}{l}\text { Intraclass } \\
\text { Correlation }^{\mathrm{b}}\end{array}$} & \multicolumn{2}{|c|}{ 95\% Confidence Interval } & \multicolumn{4}{|c|}{ F Test with True Value 0} \\
\hline & & \begin{tabular}{|l} 
Lower \\
Bound
\end{tabular} & $\begin{array}{l}\text { Upper } \\
\text { Bound }\end{array}$ & Value & df1 & df2 & Sig \\
\hline Single Measures & $.589^{\mathrm{a}}$ & .196 & .819 & 3.863 & 18 & 18 & .003 \\
\hline $\begin{array}{l}\text { Average } \\
\text { Measures }\end{array}$ & .741 & .328 & .900 & 3.863 & 18 & 18 & .003 \\
\hline
\end{tabular}

Table D6. Intraclass Correlation Coefficient for Day 1 and Day 2 left peak interval.

Intraclass Correlation Coefficient

\begin{tabular}{|c|c|c|c|c|c|c|c|}
\hline & \multirow{2}{*}{$\begin{array}{l}\text { Intraclass } \\
\text { Correlation }^{\mathrm{b}}\end{array}$} & \multicolumn{2}{|c|}{$95 \%$ Confidence Interval } & \multicolumn{4}{|c|}{ F Test with True Value 0} \\
\hline & & $\begin{array}{l}\text { Lower } \\
\text { Bound }\end{array}$ & $\begin{array}{l}\text { Upper } \\
\text { Bound }\end{array}$ & Value & df1 & df2 & Sig \\
\hline Single Measures & $.585^{\mathrm{a}}$ & .191 & .817 & 3.818 & 18 & 18 & .003 \\
\hline $\begin{array}{l}\text { Average } \\
\text { Measures }\end{array}$ & .738 & .320 & .899 & 3.818 & 18 & 18 & .003 \\
\hline
\end{tabular}


$\underline{\text { Table D7. Intraclass Correlation Coefficient for Day } 1 \text { and Day } 2 \text { left augmentation index. }}$

Intraclass Correlation Coefficient

\begin{tabular}{|c|c|c|c|c|c|c|c|}
\hline & \multirow{2}{*}{$\begin{array}{l}\text { Intraclass } \\
\text { Correlation }^{\mathrm{b}}\end{array}$} & \multicolumn{2}{|c|}{$95 \%$ Confidence Interval } & \multicolumn{4}{|c|}{ F Test with True Value 0} \\
\hline & & $\begin{array}{l}\text { Lower } \\
\text { Bound }\end{array}$ & $\begin{array}{l}\text { Upper } \\
\text { Bound }\end{array}$ & Value & df1 & df2 & Sig \\
\hline Single Measures & $.317^{\mathrm{a}}$ & -.147 & .667 & 1.929 & 18 & 18 & .087 \\
\hline $\begin{array}{l}\text { Average } \\
\text { Measures }\end{array}$ & .482 & -.346 & .800 & 1.929 & 18 & 18 & .087 \\
\hline
\end{tabular}

$\underline{\text { Table D8. Intraclass Correlation Coefficient for Day } 1 \text { and Day } 2 \text { right systolic interval. }}$

Intraclass Correlation Coefficient

\begin{tabular}{|l|l|l|l|l|l|l|l|l|}
\hline \multirow{2}{*}{ Correlation $^{\mathrm{b}}$} & \multicolumn{2}{|l|}{\begin{tabular}{l} 
Intraclass Confidence Interval \\
\cline { 3 - 9 }
\end{tabular}} & $\begin{array}{l}\text { Lower } \\
\text { Bound }\end{array}$ & $\begin{array}{l}\text { Upper } \\
\text { Bound }\end{array}$ & Value & df1 & df2 & Sig \\
\hline $\begin{array}{l}\text { Single Measures } \\
\text { Average }\end{array}$ & $.572^{\mathrm{a}}$ & .172 & .810 & 3.677 & 18 & 18 & .004 \\
Measures & .728 & .294 & .895 & 3.677 & 18 & 18 & .004 \\
\hline
\end{tabular}

Table D9. Intraclass Correlation Coefficient for Day 1 and Day 2 right systolic interval.

Intraclass Correlation Coefficient

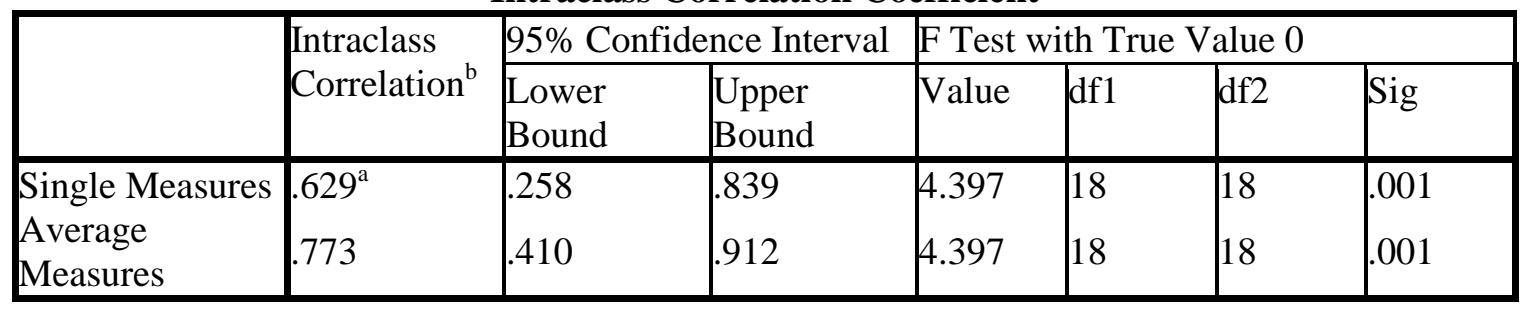

$\underline{\text { Table D10. Intraclass Correlation Coefficient for Day } 1 \text { and Day } 2 \text { right systolic interval. }}$

Intraclass Correlation Coefficient

\begin{tabular}{|c|c|c|c|c|c|c|c|}
\hline & \multirow{2}{*}{$\begin{array}{l}\text { Intraclass } \\
\text { Correlation }^{\mathrm{b}}\end{array}$} & \multicolumn{2}{|c|}{ 95\% Confidence Interval } & \multicolumn{4}{|c|}{ F Test with True Value 0} \\
\hline & & $\begin{array}{l}\text { Lower } \\
\text { Bound } \\
\end{array}$ & $\begin{array}{l}\text { Upper } \\
\text { Bound }\end{array}$ & Value & $\mathrm{df1}$ & $\mathrm{df} 2$ & Sig \\
\hline Single Measures & $.483^{\mathrm{a}}$ & .050 & .763 & 2.866 & 18 & 18 & .016 \\
\hline $\begin{array}{l}\text { Average } \\
\text { Measures }\end{array}$ & .651 & .094 & .866 & 2.866 & 18 & 18 & .016 \\
\hline
\end{tabular}


Table D11. Intraclass Correlation Coefficient for Day 1 and Day 2 right systolic interval.

Intraclass Correlation Coefficient

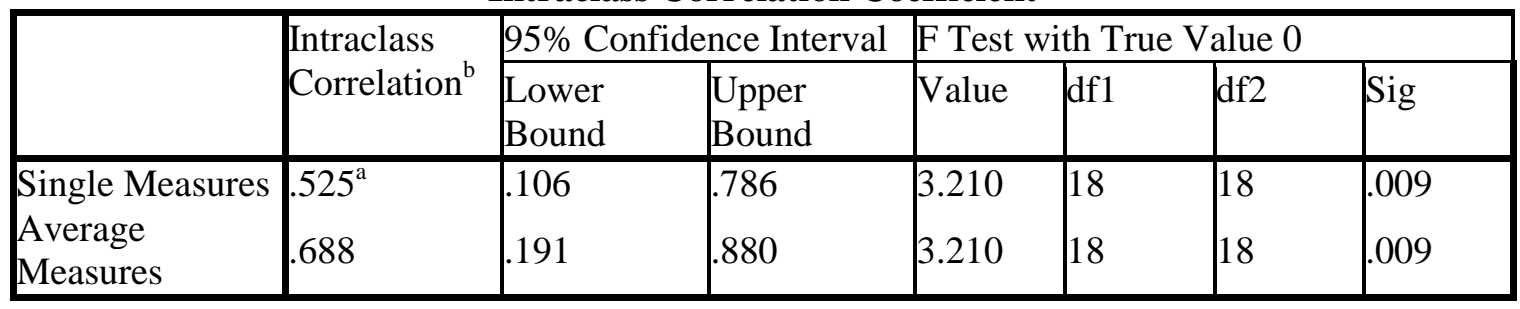

Table D12. Spearman's Rho Correlations between Day 1 and Day 2 measures for Left BAM Variables.

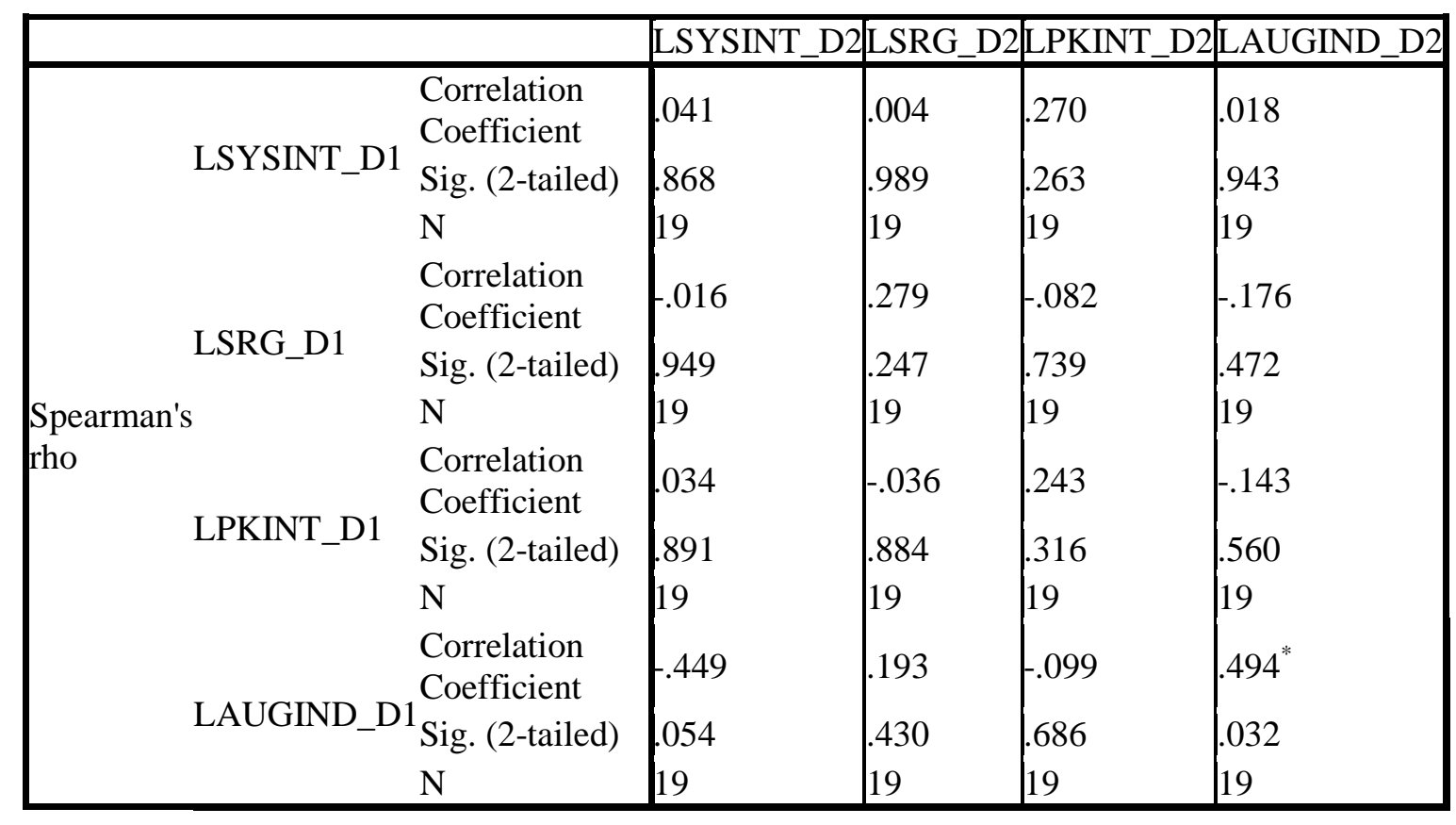


Table D13. Spearman's Rho Correlations between Day 1 and Day 2 measures for Right BAM variables.

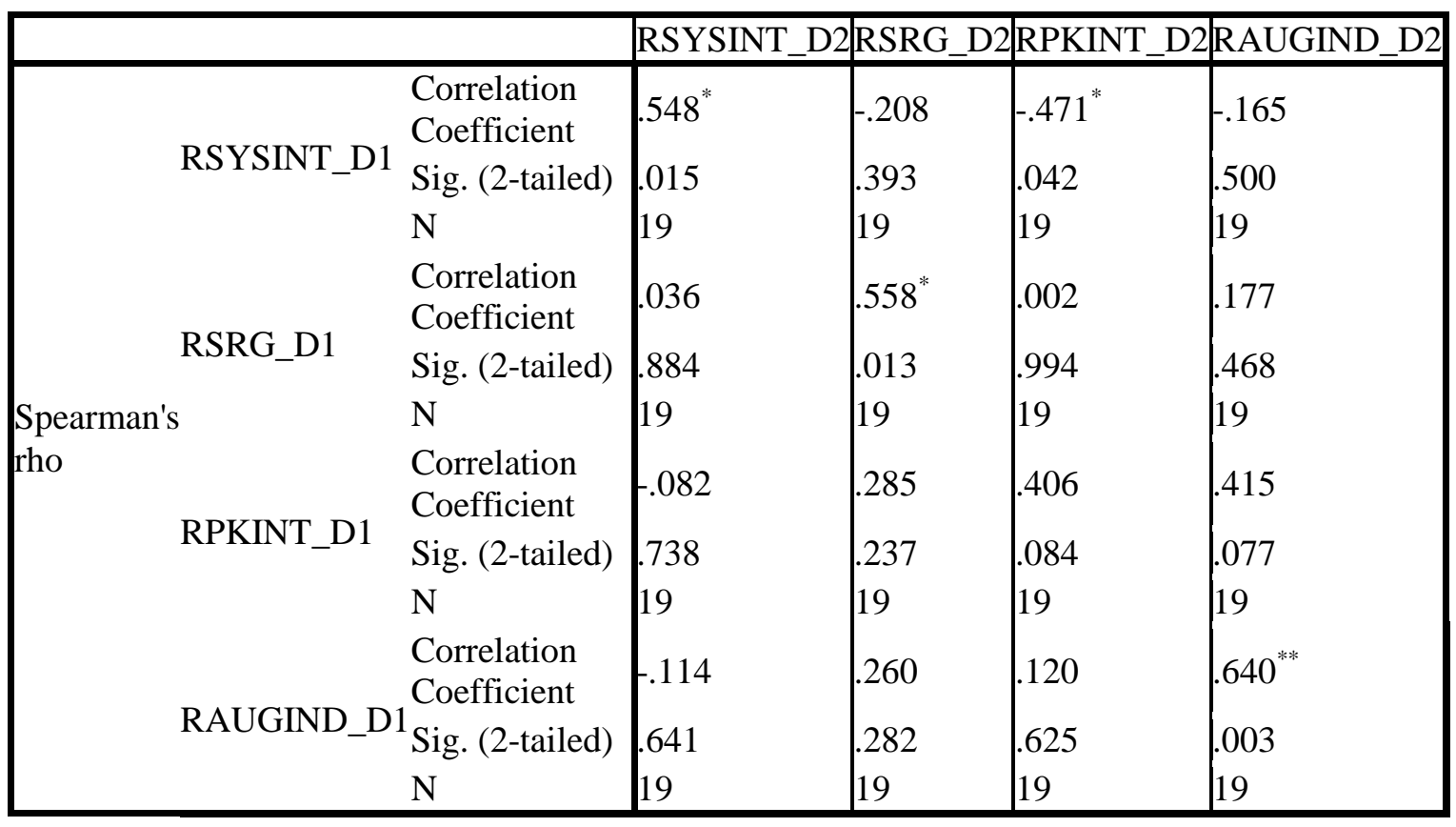

\section{Manuscript II}

Table D14. Descriptive statistics for subject demographics, sport type, history of previous concussion, and number of previous concussion for Concussed Group.

\section{Concussed Group Descriptive Statistics ${ }^{\mathrm{a}}$}

\begin{tabular}{|l|l|l|l|l|l|}
\hline & $\mathrm{N}$ & Minimum & Maximum & Mean & Std. Deviation \\
\cline { 2 - 6 } & Statistic & Statistic & Statistic & Statistic & Statistic \\
\hline Group & 17 & .00 & .00 & .0000 & .00000 \\
Age & 17 & 15.00 & 22.00 & 18.7647 & 2.10741 \\
Height & 17 & 149.86 & 264.16 & 182.1294 & 25.55183 \\
Weight & 17 & 46.27 & 113.40 & 77.1107 & 23.20757 \\
Gender & 17 & .00 & 1.00 & .4118 & .50730 \\
Sport_Type & 17 & 1.00 & 4.00 & 1.6471 & 86177 \\
CxHx & 17 & 1.00 & 1.00 & 1.0000 & .00000 \\
CxNumber & 17 & 1.00 & 3.00 & 1.2941 & .58787 \\
Valid N (listwise) & 17 & & & & \\
\hline
\end{tabular}


Table D15. Distribution (frequency) of genders, sport type, and number of previous concussion among Concussed Group.

\begin{tabular}{|ll|l|l|l|l|}
\hline & Grequency & Percent & Valid Percent & $\begin{array}{l}\text { Cumulative } \\
\text { Percent }\end{array}$ \\
\hline \multirow{2}{*}{ Valid } & Male & 10 & 58.8 & 58.8 & 58.8 \\
& Female & 7 & 41.2 & 41.2 & 100.0 \\
Total & 17 & 100.0 & 100.0 & \\
\hline
\end{tabular}

Sport_Type ${ }^{\text {a }}$

\begin{tabular}{|c|c|c|c|c|c|}
\hline & & Frequency & Percent & Valid Percent & $\begin{array}{l}\text { Cumulative } \\
\text { Percent }\end{array}$ \\
\hline \multirow{5}{*}{ Valid } & Collision & 9 & 52.9 & 52.9 & 52.9 \\
\hline & Contact & 6 & 35.3 & 35.3 & 88.2 \\
\hline & Limited Contact & 1 & 5.9 & 5.9 & 94.1 \\
\hline & Noncontact & 1 & 5.9 & 5.9 & 100.0 \\
\hline & Total & 17 & 100.0 & 100.0 & \\
\hline
\end{tabular}

\section{CxNumber ${ }^{\mathrm{a}}$}

\begin{tabular}{|c|c|c|c|c|c|}
\hline & & Frequency & Percent & Valid Percent & $\begin{array}{l}\text { Cumulative } \\
\text { Percent }\end{array}$ \\
\hline \multirow{4}{*}{ Valid } & 1.00 & 13 & 76.5 & 76.5 & 76.5 \\
\hline & 2.00 & 3 & 17.6 & 17.6 & 94.1 \\
\hline & 3.00 & 1 & 5.9 & 5.9 & 100.0 \\
\hline & Total & 17 & 100.0 & 100.0 & \\
\hline
\end{tabular}


Table D16. Descriptive statistics and tests of normality for Concussed Group ( $n=17)$ SAC, BESS, Total Symptoms, and Symptom Severity at 24-48 hours post-injury, Symptom resolution, and Return-to-Play.

\begin{tabular}{|c|c|c|c|c|c|c|c|c|c|}
\hline & \multirow{2}{*}{\begin{tabular}{|l} 
N \\
Statistic
\end{tabular}} & \multirow{2}{*}{\begin{tabular}{|l} 
Minimum \\
Statistic \\
\end{tabular}} & \multirow{2}{*}{\begin{tabular}{|l|} 
Maximum \\
Statistic \\
\end{tabular}} & \multirow{2}{*}{\begin{tabular}{|l} 
Mean \\
Statistic
\end{tabular}} & \multirow{2}{*}{\begin{tabular}{|l} 
Std. \\
Deviation \\
Statistic
\end{tabular}} & \multicolumn{2}{|c|}{ Skewness } & \multicolumn{2}{|c|}{ Kurtosis } \\
\hline & & & & & & Statisti & $\begin{array}{l}\text { Std. } \\
\text { Error }\end{array}$ & Statisti & $\begin{array}{l}\text { Std. } \\
\text { Error }\end{array}$ \\
\hline SAC_24hr & 12 & 20.00 & 29.00 & 25.0833 & 3.05877 & -.460 & .637 & -.586 & 1.232 \\
\hline BESS_24hr & 12 & 20.00 & 29.00 & 25.5000 & 3.00000 & -.824 & 637 & -.624 & 1.232 \\
\hline TS_24hr & 17 & .00 & 21.00 & 8.9412 & 6.83256 & .437 & 550 & -.737 & 1.063 \\
\hline SS_24hr & 17 & .00 & 70.00 & 22.6471 & 20.98494 & .978 & 550 & .178 & 1.063 \\
\hline SAC_SF & 15 & 25.00 & 30.00 & 27.0667 & 1.62422 & .108 & 580 & -.950 & 1.121 \\
\hline BESS_SF & 15 & 20.00 & 30.00 & 26.4000 & 2.72029 & -.808 & .580 & .754 & 1.121 \\
\hline TS_SF & 15 & .00 & 13.00 & 1.8000 & 3.50917 & 2.732 & .580 & 7.922 & 1.121 \\
\hline SS_SF & 15 & .00 & 22.00 & 2.8667 & 6.11633 & 2.724 & .580 & 7.345 & 1.121 \\
\hline SAC_RTP & 13 & 24.00 & 30.00 & 28.1538 & 2.19265 & -.959 & .616 & -.605 & 1.191 \\
\hline BESS_RTP & 13 & 26.00 & 30.00 & 28.3846 & 1.50214 & -.435 & .616 & -1.226 & 1.191 \\
\hline TS_RTP & 14 & .00 & 2.00 & .2857 & .61125 & 2.165 & .597 & 4.251 & 1.154 \\
\hline SS_RTP & 14 & .00 & 2.00 & .2857 & .61125 & 2.165 & .597 & 4.251 & 1.154 \\
\hline $\begin{array}{l}\text { Valid N } \\
\text { (listwise) }\end{array}$ & 7 & & & & & & & & \\
\hline
\end{tabular}

Table D17. Descriptive statistics for Concussed Group ( $n=17)$ time of first assessment, time to symptom resolution, and time to return-to-play.

\begin{tabular}{|l|l|l|l|l|}
\hline & $\mathrm{N}$ & Minimum & Maximum & Mean \\
\cline { 2 - 5 } & Statistic & Statistic & Statistic & Statistic \\
\hline time_to_A1 & 17 & 24.00 & 48.00 & 29.6471 \\
time_to_SF & 16 & 48.00 & 288.00 & 123.0000 \\
time_to_RTP & 15 & 120.00 & 480.00 & 244.8000 \\
Valid N (listwise) & 14 & & & \\
\hline
\end{tabular}


Table D18. Descriptive statistics for Concussed Group (n=17) Augmentation Index, Peak Interval, Systolic Interval, and Steepest Rise Gradient at 24-48 hours post-injury, Symptom resolution, and Return-to-Play.

\begin{tabular}{|l|l|l|l|l|l|}
\hline & $N$ & Minimum & Maximum & Mean & Std. Deviation \\
\cline { 2 - 6 } & Statistic & Statistic & Statistic & Statistic & Statistic \\
\hline SysInt_L24hr_avg & 17 & .09 & .23 & .1282 & .04082 \\
SysInt_LSF_avg & 17 & .06 & .23 & .1135 & .04822 \\
SysInt_LRTP_avg & 17 & .06 & .25 & .1289 & .04875 \\
SRG_L24hr_avg & 17 & 1.38 & 6.71 & 3.6249 & 1.74065 \\
SRG_LSF_avg & 17 & 1.16 & 9.50 & 3.8378 & 2.43006 \\
SRG_LRTP_avg & 17 & .21 & 5.00 & 2.7027 & 1.34134 \\
PkInt_L24hr_avg & 17 & .10 & .28 & .1776 & .06821 \\
PkInt_LSF_avg & 17 & .08 & .28 & .1780 & .07601 \\
PkInt_LRTP_avg & 17 & .06 & .31 & .1661 & .07890 \\
Aix_L24hr_avg & 17 & .48 & 44.87 & 21.9015 & 13.82417 \\
Aix_LSF_avg & 17 & .00 & 57.04 & 23.5399 & 17.98367 \\
Aix_LRTP_avg & 17 & .00 & 55.91 & 22.3491 & 17.49184 \\
SysInt_R24hr_avg & 17 & .07 & .23 & .1327 & .04626 \\
SysInt_RSF_avg & 17 & .08 & .24 & .1246 & .03616 \\
SysInt_RRTP_avg & 17 & .07 & .24 & .1266 & .04418 \\
SRG_R24hr_avg & 17 & 1.40 & 6.24 & 3.1349 & 1.51533 \\
SRG_RSF_avg & 17 & 1.33 & 11.83 & 3.5278 & 2.56299 \\
SRG_RRTP_avg & 17 & 1.10 & 9.32 & 2.9942 & 2.01147 \\
PkInt_R24hr_avg & 17 & .06 & .28 & .1857 & .08344 \\
PkInt_RSF_avg & 17 & .04 & .29 & .1821 & .08871 \\
PkInt_RRTP_avg & 17 & .08 & .28 & .1964 & .06801 \\
Aix_R24hr_avg & 17 & .00 & 68.44 & 19.9833 & 18.69202 \\
Aix_RSF_avg & 17 & .00 & 63.56 & 27.7500 & 22.76985 \\
Aix_RRTP_avg & 17 & .00 & 77.16 & 28.6626 & 19.68088 \\
Valid N(listwise) & 17 & & & & \\
\hline
\end{tabular}

Table D19. Friedman's Rank Test - Left Systolic Interval (Concussed Group) differences at 24$48 \mathrm{hrs}$ post-injury, symptom resolution, and return-to-activity

Ranks

\begin{tabular}{|l|l|}
\hline & Mean Rank \\
\hline SysInt_L24hr_avg & 2.18 \\
SysInt_LSF_avg & 1.71 \\
SysInt_LRTP_avg & 2.12 \\
\hline
\end{tabular}

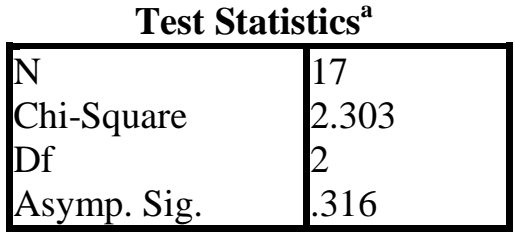

a. Friedman Test 
Table D20. Friedman's Rank Test - Left Steepest Rise Gradient (Concussed Group) differences at $24-48$ hrs post-injury, symptom resolution, and return-to-activity

\section{Ranks}

\begin{tabular}{|l|l|}
\hline & Mean Rank \\
\hline SRG_L24hr_avg & 2.12 \\
SRG_LSF_avg & 2.18 \\
SRG_LRTP_avg & 1.71 \\
\hline
\end{tabular}

\begin{tabular}{|l|l|}
\hline \multicolumn{2}{|c|}{ Test Statistics } \\
\hline N \\
Chi-Square & 17 \\
Df & 2.235 \\
Asymp. Sig. & 2 \\
\hline
\end{tabular}

a. Friedman Test

Table D21. Friedman's Rank Test - Left Peak Interval (Concussed Group) differences at 24-48 hrs post-injury, symptom resolution, and return-to-activity

\section{Ranks}

\begin{tabular}{|l|l|}
\hline & Mean Rank \\
\hline PkInt_L24hr_avg & 1.94 \\
PkInt_LSF_avg & 2.24 \\
PkInt_LRTP_avg & 1.82 \\
\hline
\end{tabular}

Test Statistics
\begin{tabular}{|l|l|}
\hline $\mathrm{N}$ & 17 \\
Chi-Square & 1.529 \\
Df & 2 \\
Asymp. Sig. & .465 \\
\hline
\end{tabular}

a. Friedman Test

Table D22. Friedman's Rank Test - Left Augmentation Index (Concussed Group) differences at 24-48 hrs post-injury, symptom resolution, and return-to-activity

\section{Ranks}

\begin{tabular}{|l|l|}
\hline & Mean Rank \\
\hline Aix_L24hr_avg & 1.88 \\
Aix_LSF_avg & 2.18 \\
Aix_LRTP_avg & 1.94 \\
\hline
\end{tabular}

\section{Test Statistics ${ }^{\mathrm{a}}$}

\begin{tabular}{|l|l|}
\hline N & 17 \\
Chi-Square & .824 \\
Df & 2 \\
Asymp. Sig. & .662 \\
\hline
\end{tabular}

a. Friedman Test

Table D23. Friedman's Rank Test - Right Systolic Interval (Concussed Group) differences at 24$48 \mathrm{hrs}$ post-injury, symptom resolution, and return-to-activity

Ranks
\begin{tabular}{|l|l|}
\hline & Mean Rank \\
\hline SysInt_R24hr_avg & 2.26 \\
SysInt_RSF_avg & 2.00 \\
SysInt_RRTP_avg & 1.74 \\
\hline
\end{tabular}

Test Statistics ${ }^{\mathrm{a}}$
\begin{tabular}{|l|l|}
\hline $\mathrm{N}$ & 17 \\
Chi-Square & 2.455 \\
Df & 2 \\
Asymp. Sig. & .293 \\
\hline
\end{tabular}


Table D24. Friedman's Rank Test - Right Steepest Rise Gradient (Concussed Group) differences at $24-48$ hrs post-injury, symptom resolution, and return-to-activity

Ranks
\begin{tabular}{|l|l|}
\hline & Mean Rank \\
\hline SRG_R24hr_avg & 2.00 \\
SRG_RSF_avg & 2.24 \\
SRG_RRTP_avg & 1.76 \\
\hline
\end{tabular}

Test Statistics $^{\mathrm{a}}$
\begin{tabular}{|l|l|}
\hline $\mathrm{N}$ & 17 \\
Chi-Square & 1.882 \\
df & 2 \\
Asymp. Sig. & 390 \\
\hline
\end{tabular}

a. Friedman Test

Table D25. Friedman's Rank Test - Right Pulse Interval (Concussed Group) differences at 24-48 hrs post-injury, symptom resolution, and return-to-activity

Ranks
\begin{tabular}{|l|l|}
\hline & Mean Rank \\
\hline PkInt_R24hr_avg & 1.85 \\
PkInt_RSF_avg & 2.00 \\
PkInt_RRTP_avg & 2.15 \\
\hline
\end{tabular}

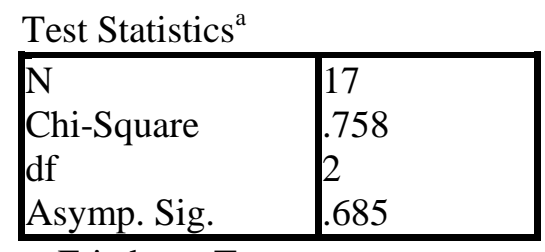

a. Friedman Test

Table D26. Friedman's Ranke Test - Right Augmentation Index (Concussed Group) differences at 24-48 hrs post-injury, symptom resolution, and return-to-activity

\section{Ranks}

\begin{tabular}{|l|l|}
\hline & Mean Rank \\
\hline Aix_R24hr_avg & 1.76 \\
Aix_RSF_avg & 2.06 \\
Aix_RRTP_avg & 2.18 \\
\hline
\end{tabular}

Test Statistics $^{\mathbf{a}}$

\begin{tabular}{|l|l|}
\hline N & 17 \\
Chi-Square & 1.529 \\
df & 2 \\
Asymp. Sig. & .465 \\
\hline
\end{tabular}

a. Friedman Test

Table D27. Friedman's Rank Test - Standardized Assessment of Concussion (Concussed Group) differences at 24-48 hrs post-injury, symptom resolution, and return-to-activity

\section{Ranks}

\begin{tabular}{|l|l|}
\hline & Mean Rank \\
\hline SAC_24hr & 1.21 \\
SAC_SF & 2.07 \\
SAC_RTP & 2.71 \\
\hline
\end{tabular}

\section{Test Statistics ${ }^{\mathrm{a}}$}

\begin{tabular}{|l|l|}
\hline N & 7 \\
Chi-Square & 8.880 \\
df & 2 \\
Asymp. Sig. & .012 \\
\hline
\end{tabular}

a. Friedman Test 
Table D28. Wilkcoxon Signed-Rank Test for Related Samples - Standardized Assessment of Concussion (Concussed Group) differences at $24-48 \mathrm{hrs}$ post-injury, symptom resolution, and return-to-activity

\section{Ranks}

\begin{tabular}{|ll|l|l|l|}
\hline & & N & Mean Rank & Sum of Ranks \\
\hline SAC_SF - SAC_24hr & Negative Ranks & $2^{\mathrm{a}}$ & 2.25 & 4.50 \\
& Positive Ranks & $7^{\mathrm{b}}$ & 5.79 & 40.50 \\
& Ties & $1^{\mathrm{c}}$ & & \\
& Total & 10 & 5.50 & 11.00 \\
& Negative Ranks & $2^{\mathrm{d}}$ & & \\
& Positive Ranks & $8^{\mathrm{e}}$ & 5.50 & \\
SAC_RTP - SAC_SF & Ties & $2^{\mathrm{f}}$ & & \\
& Total & 12 & \\
& Negative Ranks & $0^{\mathrm{g}}$ & .00 & \\
& Positive Ranks & $8^{\mathrm{h}}$ & 4.50 & 36.00 \\
SAC_RTP - SAC_24hr & Ties & $0^{\mathrm{i}}$ & & \\
& Total & 8 & & \\
\hline
\end{tabular}

\section{Test Statistics ${ }^{\mathrm{a}}$}

\begin{tabular}{|l|l|l|l|}
\hline & SAC_SF - SAC_24hr & SAC_RTP - SAC_SF & SAC_RTP - SAC_24hr \\
\hline Z & $-2.136^{\mathrm{b}}$ & $-1.705^{\mathrm{b}}$ & $-2.533^{\mathrm{b}}$ \\
Asymp. Sig. (2-tailed) & .033 & .088 & .011 \\
\hline
\end{tabular}

a. Wilcoxon Signed Ranks Test

b. Based on negative ranks.

Table D29. Friedman's Rank Test - Modified Balance Error Scoring System (Concussed Group) differences at 24-48 hrs post-injury, symptom resolution, and return-to-activity

\section{Ranks}

\begin{tabular}{|l|l|}
\hline & Mean Rank \\
\hline BESS_24hr & 1.71 \\
BESS_SF & 1.64 \\
BESS_RTP & 2.64 \\
\hline
\end{tabular}

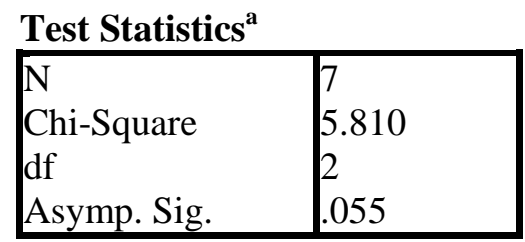

a. Friedman Test

Table D30. Friedman's Rank Test - Total Symptom (Concussed Group) differences at 24-48 hrs post-injury, symptom resolution, and return-to-activity

\section{Ranks}

\begin{tabular}{|l|l|}
\hline & Mean Rank \\
\hline TS_24hr & 2.88 \\
TS_SF & 1.81 \\
TS_RTP & 1.31 \\
\hline
\end{tabular}

Test Statistics
\begin{tabular}{|l|l|}
\hline $\mathrm{N}$ & 13 \\
Chi-Square & 21.415 \\
Df & 2 \\
Asymp. Sig. & .000 \\
\hline
\end{tabular}

a. Friedman Test 
Table D31. Wilkcoxon Signed-Rank Test for Related Samples - Total Symptom (Concussed Group) differences at 24-48 hrs post-injury, symptom resolution, and return-to-activity

\begin{tabular}{|ll|l|l|l|}
\hline Ranks & N & Mean Rank & Sum of Ranks \\
\hline & Negative Ranks & $13^{\mathrm{a}}$ & 7.00 & 91.00 \\
TS_SF - TS_24hr & Positive Ranks & $0^{\mathrm{b}}$ & .00 & .00 \\
& Ties & $2^{\mathrm{c}}$ & & \\
& Total & 15 & 3.50 & 21.00 \\
& Negative Ranks & $6^{\mathrm{d}}$ & .00 & .00 \\
TS_RTP - TS_SF & Positive Ranks & $0^{\mathrm{e}}$ & & \\
& Ties & $7^{\mathrm{f}}$ & 7.00 & 91.00 \\
& Total & 13 & .00 & .00 \\
& Negative Ranks & $13^{\mathrm{g}}$ & & \\
TS_RTP - TS_24hr & Positive Ranks & $0^{\mathrm{h}}$ & & \\
& Ties & $1^{\mathrm{i}}$ & & \\
& Total & 14 & & \\
\end{tabular}

\section{Test Statistics ${ }^{\mathrm{a}}$}

\begin{tabular}{|l|l|l|l|}
\hline & TS_SF - TS_24hr & TS_RTP - TS_SF & TS_RTP - TS_24hr \\
\hline Z & $-3.185^{\mathrm{b}}$ & $-2.214^{\mathrm{b}}$ & $-3.184^{\mathrm{b}}$ \\
Asymp. Sig. (2-tailed) & .001 & .027 & .001 \\
\hline
\end{tabular}

a. Wilcoxon Signed Ranks Test

b. Based on positive ranks.

Table D32. Friedman's Rank Test - Symptom Severity (Concussed Group) differences at 24-48 hrs post-injury, symptom resolution, and return-to-activity

\section{Ranks}

\begin{tabular}{|l|l|}
\hline & Mean Rank \\
\hline SS_24hr & 2.88 \\
SS_SF & 1.81 \\
SS_RTP & 1.31 \\
\hline
\end{tabular}

Test Statistics
\begin{tabular}{|l|l|}
\hline N \\
Chi-Square & 13 \\
df & 21.415 \\
Asymp. Sig. & 2 \\
\hline
\end{tabular}

a. Friedman Test 
Table D133. Wilcoxon Signed-Rank Test for Related Samples - Total Symptom (Concussed Group) differences at $24-48$ hrs post-injury, symptom resolution, and return-to-activity

\begin{tabular}{|ll|l|l|l|}
\hline Ranks & N & Mean Rank & Sum of Ranks \\
\hline & Negative Ranks & $13^{\mathrm{a}}$ & 7.00 & 91.00 \\
SS_SF - SS_24hr & Positive Ranks & $0^{\mathrm{b}}$ & .00 & .00 \\
& Ties & $2^{\mathrm{c}}$ & & \\
& Total & 15 & 3.50 & 21.00 \\
& Negative Ranks & $6^{\mathrm{d}}$ & .00 & .00 \\
SS_RTP - SS_SF & Positive Ranks & $0^{\mathrm{e}}$ & & \\
& Ties & $7^{\mathrm{f}}$ & 7.00 & 91.00 \\
& Total & 13 & .00 & .00 \\
& Negative Ranks & $13^{\mathrm{g}}$ & & \\
SS_RTP - SS_24hr & Positive Ranks & $0^{\mathrm{h}}$ & & \\
& Ties & $1^{\mathrm{i}}$ & & \\
& Total & 14 & & \\
\end{tabular}

\section{Test Statistics $^{\mathrm{a}}$}

\begin{tabular}{|l|l|l|l|}
\hline & SS_SF - SS_24hr & SS_RTP - SS_SF & SS_RTP - SS_24hr \\
\hline$Z$ & $-3.180^{\mathrm{b}}$ & $-2.226^{\mathrm{b}}$ & $-3.182^{\mathrm{b}}$ \\
Asymp. Sig. (2-tailed) & .001 & .026 & .001 \\
\hline
\end{tabular}

a. Wilcoxon Signed Ranks Test

b. Based on positive ranks. 
Table D34. Spearman's Rho Correlations between Time to Resolution of Symptoms and Left and Right Systolic Interval, Steepest Rise Gradient, Peak Interval, and Augmentation Index.

\section{Correlations}

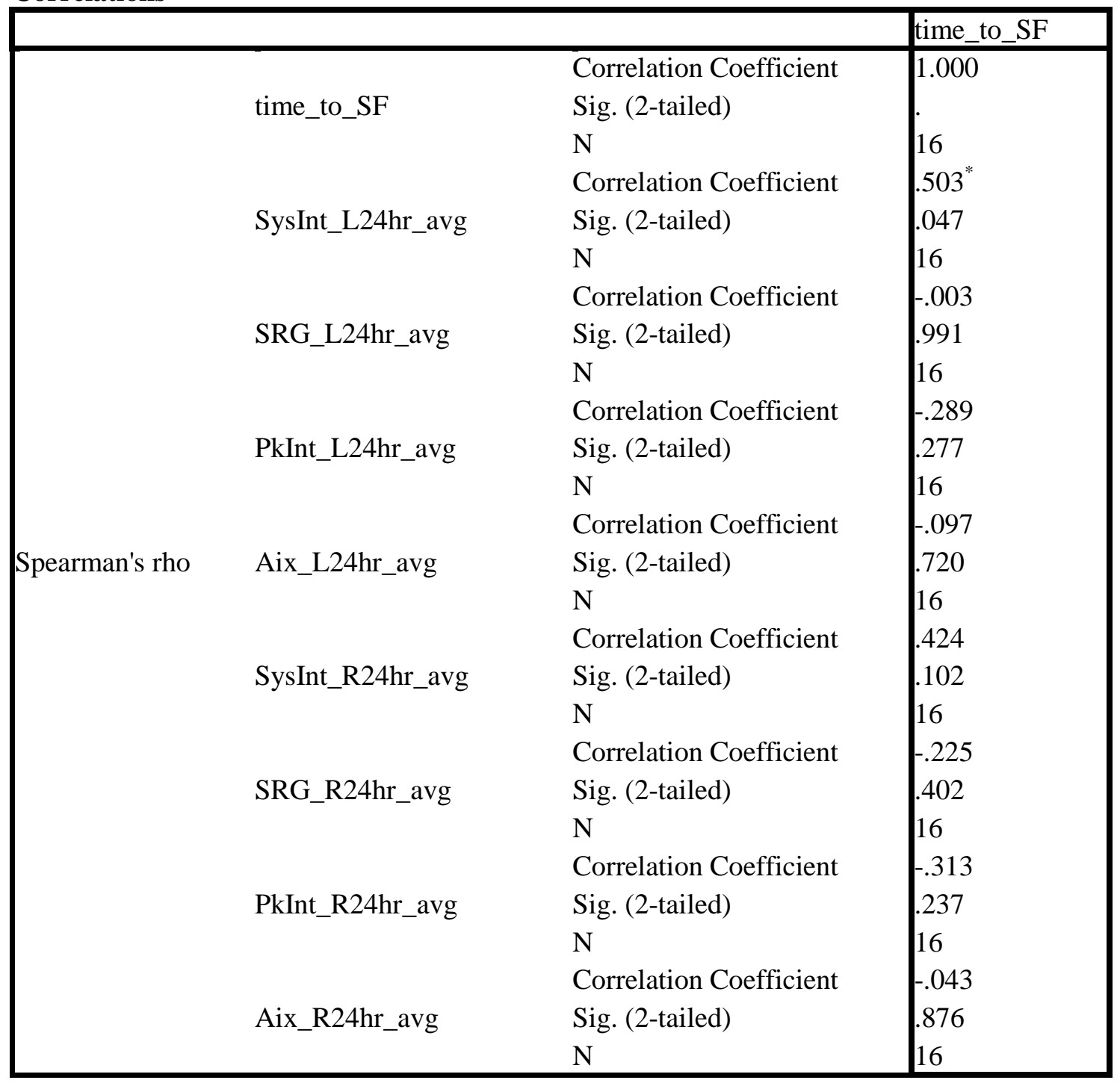


Table D35. Spearman's Rho Correlations between Time to Return to Play and Left and Right Systolic Interval, Steepest Rise Gradient, Peak Interval, and Augmentation Index.

\section{Correlations}

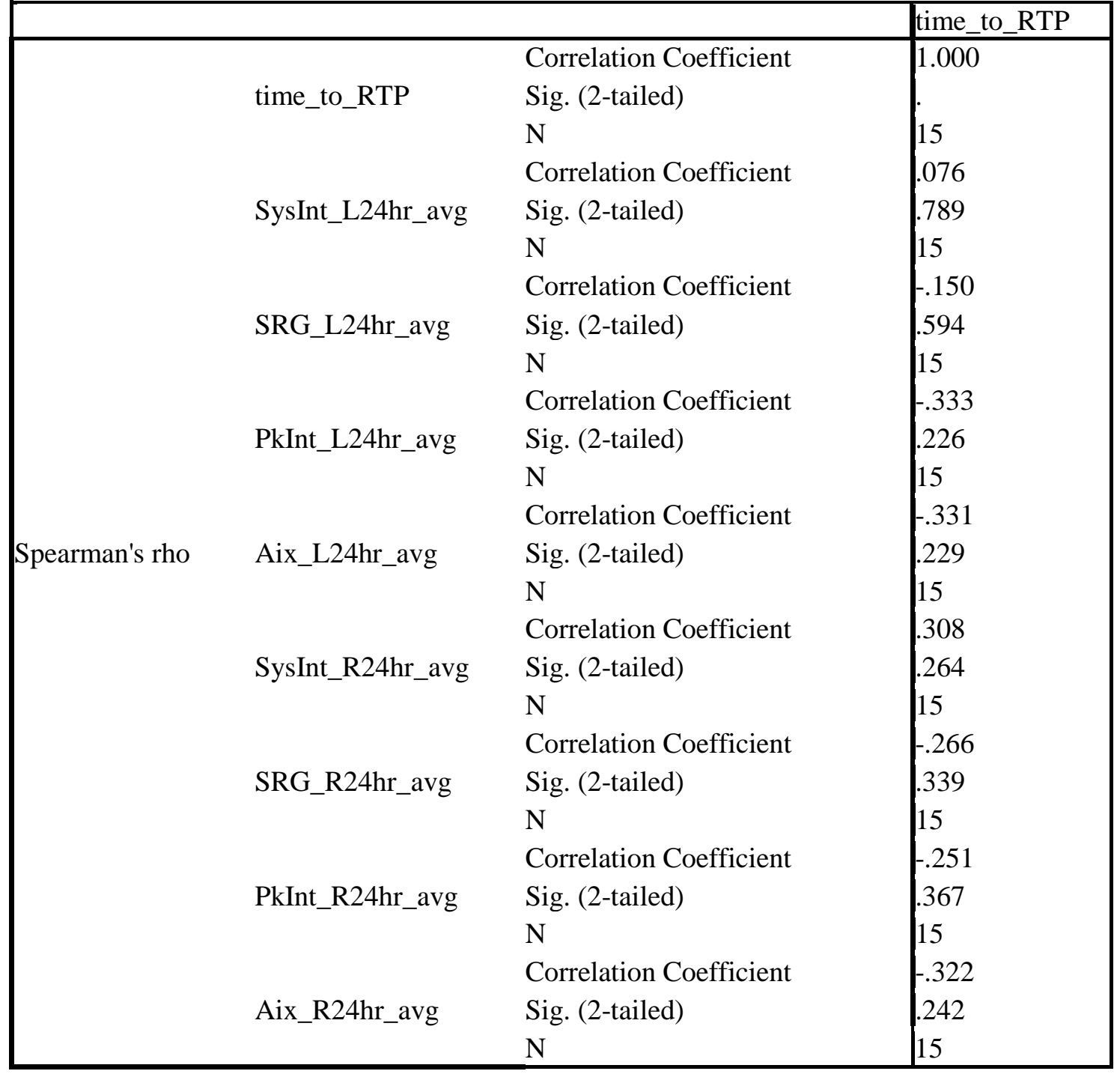


Table D36. Descriptive statistics for subject demographics, sport type, concussion history, and number of prior concussions for Concussed and Healthy Groups.

Concussed Group Descriptive Statistics ${ }^{\mathrm{a}}$

\begin{tabular}{|l|l|l|l|l|l|}
\hline & $\mathrm{N}$ & Minimum & Maximum & Mean & Std. Deviation \\
\cline { 2 - 6 } & Statistic & Statistic & Statistic & Statistic & Statistic \\
\hline Group & 17 & .00 & .00 & .0000 & .00000 \\
Age & 17 & 15.00 & 22.00 & 18.7647 & 2.10741 \\
Height & 17 & 149.86 & 264.16 & 182.1294 & 25.55183 \\
Weight & 17 & 46.27 & 113.40 & 77.1107 & 23.20757 \\
Gender & 17 & .00 & 1.00 & .4118 & .50730 \\
Sport_Type & 17 & 1.00 & 4.00 & 1.6471 & .86177 \\
CxHx & 17 & 1.00 & 1.00 & 1.0000 & .00000 \\
CxNumber & 17 & 1.00 & 3.00 & 1.2941 & .58787 \\
Valid N (listwise) & 17 & & & & \\
\hline
\end{tabular}

a. Group $=$ Concussed

Healthy Group Descriptive Statistics ${ }^{\mathrm{a}}$

\begin{tabular}{|l|l|l|l|l|l|}
\hline \multirow{2}{*}{} & $\mathrm{N}$ & Minimum & Maximum & Mean & Std. Deviation \\
\cline { 2 - 6 } & Statistic & Statistic & Statistic & Statistic & Statistic \\
\hline Group & 17 & 1.00 & 1.00 & 1.0000 & .00000 \\
Age & 17 & 15.00 & 21.00 & 18.8824 & 1.76360 \\
Height & 17 & 160.02 & 198.12 & 176.4553 & 11.25644 \\
Weight & 17 & 52.16 & 133.81 & 75.9199 & 20.61061 \\
Gender & 17 & .00 & 1.00 & .4118 & .50730 \\
Sport_Type & 17 & 1.00 & 2.00 & 1.4118 & .50730 \\
CxHx & 17 & .00 & 1.00 & .1176 & .33211 \\
CxNumber & 17 & .00 & 3.00 & .2353 & .75245 \\
Valid N (listwise) & 17 & & & & \\
\hline
\end{tabular}

a. Group = Healthy

Table D37. Distribution (frequency) of genders, sport type, and number of previous concussion among Concussed Group.

Concussed Group: Gender

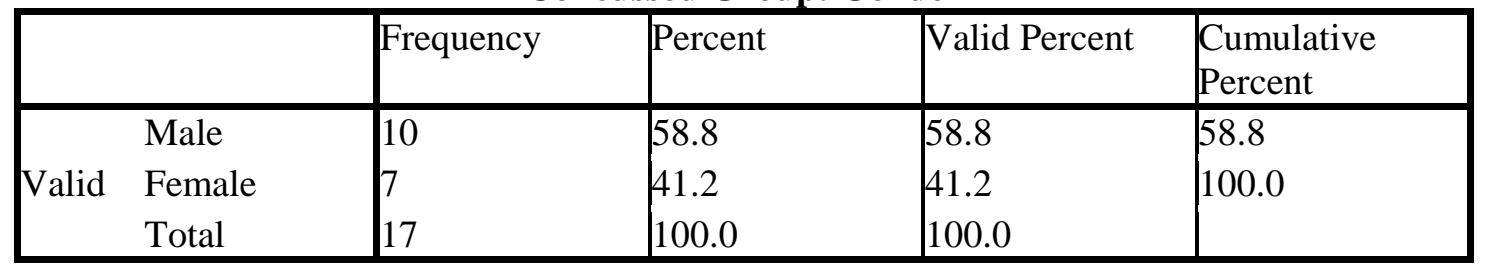

a. Group $=$ Concussed 
Healthy Group: Gender ${ }^{\mathrm{a}}$

\begin{tabular}{|ll|l|l|l|l|}
\hline & Frequency & Percent & Valid Percent & $\begin{array}{l}\text { Cumulative } \\
\text { Percent }\end{array}$ \\
\hline \multirow{2}{*}{ Valid } & Male & 10 & 58.8 & 58.8 & 58.8 \\
& Female & 7 & 41.2 & 41.2 & 100.0 \\
& Total & 17 & 100.0 & 100.0 & \\
\hline
\end{tabular}

a. Group = Healthy

\section{Concussed Group: Sport_Type ${ }^{\mathrm{a}}$}

\begin{tabular}{|c|c|c|c|c|c|}
\hline & & Frequency & Percent & Valid Percent & $\begin{array}{l}\text { Cumulative } \\
\text { Percent }\end{array}$ \\
\hline \multirow{5}{*}{ Valid } & Collision & 9 & 52.9 & 52.9 & 52.9 \\
\hline & Contact & 6 & 35.3 & 35.3 & 88.2 \\
\hline & Limited Contact & 1 & 5.9 & 5.9 & 94.1 \\
\hline & Noncontact & 1 & 5.9 & 5.9 & 100.0 \\
\hline & Total & 17 & 100.0 & 100.0 & \\
\hline
\end{tabular}

a. Group $=$ Concussed

\section{Healthy Group: Sport_Type ${ }^{\text {a }}$}

\begin{tabular}{|ll|l|l|l|l|}
\hline & Frequency & Percent & Valid Percent & $\begin{array}{l}\text { Cumulative } \\
\text { Percent }\end{array}$ \\
\hline \multirow{2}{*}{\begin{tabular}{ll|l|l} 
Valid \\
Collision
\end{tabular} Contact } & 10 & 58.8 & 58.8 & 58.8 \\
& Total & 7 & 41.2 & 41.2 & 100.0 \\
\hline
\end{tabular}

a. Group = Healthy

\section{Concussed Group: Number of Previous Concussions}

\begin{tabular}{|ll|l|l|l|l|}
\hline & & Frequency & Percent & Valid Percent & $\begin{array}{l}\text { Cumulative } \\
\text { Percent }\end{array}$ \\
\hline \multirow{4}{*}{ Valid } & 1.00 & 13 & & & 76.5 \\
& 2.00 & 3 & 16.5 & 76.5 & 94.1 \\
& 3.00 & 1 & 17.6 & 17.6 & 100.0 \\
Total & 17 & 5.9 & 5.9 & \\
\hline
\end{tabular}

a. Group $=$ Concussed

Healthy Group Number of Previous Concussions

\begin{tabular}{|ll|l|l|l|l|}
\hline & & Frequency & Percent & Valid Percent & $\begin{array}{l}\text { Cumulative } \\
\text { Percent }\end{array}$ \\
\hline \multirow{4}{*}{ Valid } & .00 & & & & 88.2 \\
& 1.00 & 15 & 88.2 & 88.2 & 94.1 \\
& 3.00 & 1 & 5.9 & 5.9 & 100.0 \\
Total & 17 & 5.9 & 5.9 & \\
\hline
\end{tabular}

a. Group = Healthy 
Table D38. Descriptive statistics for Left Systolic Interval, Steepest Rise Gradient, Peak Interval, and Augmentation Index for Concussed Group at 24-48 hours post-injury, symptom resolution, and Return-to-Play and the Healthy group at baseline.

Concussed Group

\begin{tabular}{|l|l|l|l|l|l|}
\hline & N & Minimum & Maximum & Mean & Std. Deviation \\
\cline { 2 - 6 } & Statistic & Statistic & Statistic & Statistic & Statistic \\
\hline SysInt_L24hr_avg & 17 & .09 & .23 & .1282 & .04082 \\
SysInt_LSF_avg & 17 & .06 & .23 & .1135 & .04822 \\
SysInt_LRTP_avg & 17 & .06 & .25 & .1289 & .04875 \\
SRG_L24hr_avg & 17 & 1.38 & 6.71 & 3.6249 & 1.74065 \\
SRG_LSF_avg & 17 & 1.16 & 9.50 & 3.8378 & 2.43006 \\
SRG_LRTP_avg & 17 & .21 & 5.00 & 2.7027 & 1.34134 \\
PkInt_L24hr_avg & 17 & .10 & .28 & .1776 & .06821 \\
PkInt_LSF_avg & 17 & .08 & .28 & .1780 & .07601 \\
PkInt_LRTP_avg & 17 & .06 & .31 & .1661 & .07890 \\
Aix_L24hr_avg & 17 & .48 & 44.87 & 21.9015 & 13.82417 \\
Aix_LSF_avg & 17 & .00 & 57.04 & 23.5399 & 17.98367 \\
Aix_LRTP_avg & 17 & .00 & 55.91 & 22.3491 & 17.49184 \\
Valid N (listwise) & 17 & & & & \\
\hline
\end{tabular}

Healthy Group

\begin{tabular}{|l|l|l|l|l|l|}
\hline & $\mathrm{N}$ & Minimum & Maximum & Mean & Std. Deviation \\
\cline { 2 - 6 } & Statistic & Statistic & Statistic & Statistic & Statistic \\
\hline SysInt_baseline & 17 & .06 & .18 & .1096 & .02924 \\
SRG_baseline & 17 & .13 & 10.13 & 3.4950 & 2.84651 \\
PkInt_baseline & 17 & .10 & .28 & .1706 & .05788 \\
Aix_baseline & 17 & .08 & 76.98 & 26.3128 & 19.82663 \\
Valid N (listwise) & 17 & & & & \\
\hline
\end{tabular}

Table D39. Mann-Whitney U Test - Left Systolic Interval differences between Concussed and Healthy Groups

\begin{tabular}{|c|c|c|c|c|}
\hline \multicolumn{5}{|c|}{ Ranks } \\
\hline & Group & $\mathrm{N}$ & Mean Rank & Sum of Ranks \\
\hline \multirow{4}{*}{ SysInt_L24hr_avg } & Concussed & 17 & 19.91 & 338.50 \\
\hline & Healthy & 17 & 15.09 & 256.50 \\
\hline & Total & 34 & & \\
\hline & Concussed & 17 & 16.44 & 279.50 \\
\hline \multirow[t]{3}{*}{ SysInt_LSF_avg } & Healthy & 17 & 18.56 & 315.50 \\
\hline & Total & 34 & & \\
\hline & Concussed & 17 & 19.21 & 326.50 \\
\hline \multirow[t]{2}{*}{ SysInt_LRTP_avg } & Healthy & 17 & 15.79 & 268.50 \\
\hline & Total & 34 & & \\
\hline
\end{tabular}


Test Statistics ${ }^{\mathrm{a}}$

\begin{tabular}{|l|l|l|l|}
\hline & SysInt_L24hr_avg & SysInt_LSF_avg & SysInt_LRTP_avg \\
\hline Mann-Whitney U & 103.500 & 126.500 & 115.500 \\
Wilcoxon W & 256.500 & 279.500 & 268.500 \\
Z & -1.415 & -621 & -1.000 \\
Asymp. Sig. (2-tailed) & 157 & .535 & .317 \\
Exact Sig. [2*(1-tailed Sig.)] & $.160^{\mathrm{b}}$ & $.540^{\mathrm{b}}$ & $.322^{\mathrm{b}}$ \\
\hline
\end{tabular}

a. Grouping Variable: Group

b. Not corrected for ties.

Table D40. Mann-Whitney U Test - Left Steepest Rise Gradient differences between Concussed and Healthy Groups

\section{Ranks}

\begin{tabular}{|ll|l|l|l|}
\hline & Group & N & Mean Rank & Sum of Ranks \\
\hline \multirow{3}{*}{ SRG_L24hr_avg } & Concussed & 17 & 18.59 & 316.00 \\
& Healthy & 17 & 16.41 & 279.00 \\
& Total & 34 & & \\
& Concussed & 17 & 18.59 & 316.00 \\
SRG_LSF_avg & Healthy & 17 & 16.41 & 279.00 \\
& Total & 34 & & \\
& Concussed & 17 & 16.88 & 287.00 \\
SRG_LRTP_avg & Healthy & 17 & 18.12 & 308.00 \\
& Total & 34 & & \\
\hline
\end{tabular}

Test Statistics ${ }^{\mathrm{a}}$

\begin{tabular}{|l|l|l|l|}
\hline & SRG_L24hr_avg & SRG_LSF_avg & SRG_LRTP_avg \\
\hline Mann-Whitney U & 126.000 & 126.000 & 134.000 \\
Wilcoxon W & 279.000 & 279.000 & 287.000 \\
Z & .637 & -637 & -.362 \\
Asymp. Sig. (2-tailed) & .524 & .524 & .718 \\
Exact Sig. [2*(1-tailed Sig.)] & $.540^{\mathrm{b}}$ & $.540^{\mathrm{b}}$ & $.734^{\mathrm{b}}$ \\
\hline
\end{tabular}

a. Grouping Variable: Group

b. Not corrected for ties. 
Table D41. Mann-Whitney U Test - Left Peak Interval differences between Concussed and Healthy Groups

\begin{tabular}{|c|c|c|c|c|}
\hline \multicolumn{5}{|c|}{ Ranks } \\
\hline & Group & $\mathrm{N}$ & Mean Rank & Sum of Ranks \\
\hline \multirow{4}{*}{ PkInt_L24hr_avg } & Concussed & 17 & 17.56 & 298.50 \\
\hline & Healthy & 17 & 17.44 & 296.50 \\
\hline & Total & 34 & & \\
\hline & Concussed & 17 & 17.41 & 296.00 \\
\hline \multirow[t]{3}{*}{ PkInt_LSF_avg } & Healthy & 17 & 17.59 & 299.00 \\
\hline & Total & 34 & & \\
\hline & Concussed & 17 & 16.15 & 274.50 \\
\hline \multirow[t]{2}{*}{ PkInt_LRTP_avg } & Healthy & 17 & 18.85 & 320.50 \\
\hline & Total & 34 & & \\
\hline
\end{tabular}

Test Statistics ${ }^{\mathrm{a}}$

\begin{tabular}{|l|l|l|l|}
\hline & PkInt_L24hr_avg & PkInt_LSF_avg & PkInt_LRTP_avg \\
\hline Mann-Whitney U & 143.500 & 143.000 & 121.500 \\
Wilcoxon W & 296.500 & 296.000 & 274.500 \\
Z & -.034 & -.052 & -.793 \\
Asymp. Sig. (2-tailed) & .973 & .959 & .428 \\
Exact Sig. [2*(1-tailed Sig.)] & $.973^{\mathrm{b}}$ & $.973^{\mathrm{b}}$ & $.433^{\mathrm{b}}$ \\
\hline
\end{tabular}

a. Grouping Variable: Group

b. Not corrected for ties.

Table D42. Mann-Whitney U Test - Left Augmentation Index differences between Concussed and Healthy Groups

\begin{tabular}{|c|c|c|c|c|}
\hline \multicolumn{5}{|c|}{ Ranks } \\
\hline & Group & $\mathrm{N}$ & Mean Rank & Sum of Ranks \\
\hline \multirow{4}{*}{ Aix_L24hr_avg } & Concussed & 17 & 15.88 & 270.00 \\
\hline & Healthy & 17 & 19.12 & 325.00 \\
\hline & Total & 34 & & \\
\hline & Concussed & 17 & 16.82 & 286.00 \\
\hline \multirow[t]{3}{*}{ Aix_LSF_avg } & Healthy & 17 & 18.18 & 309.00 \\
\hline & Total & 34 & & \\
\hline & Concussed & 17 & 17.00 & 289.00 \\
\hline \multirow[t]{2}{*}{ Aix_LRTP_avg } & Healthy & 17 & 18.00 & 306.00 \\
\hline & Total & 34 & & \\
\hline
\end{tabular}


Test Statistics ${ }^{\mathrm{a}}$

\begin{tabular}{|l|l|l|l|}
\hline & Aix_L24hr_avg & Aix_LSF_avg & Aix_LRTP_avg \\
\hline Mann-Whitney U & 117.000 & 133.000 & 136.000 \\
Wilcoxon W & 270.000 & 286.000 & 289.000 \\
Z & -.947 & -396 & -293 \\
Asymp. Sig. (2-tailed) & .344 & .692 & .770 \\
Exact Sig. [2*(1-tailed Sig.)] & $.357^{\mathrm{b}}$ & $.708^{\mathrm{b}}$ & $.786^{\mathrm{b}}$ \\
\hline
\end{tabular}

a. Grouping Variable: Group

Table D43. Descriptive statistics for Right Systolic Interval for Concussed Group at 24-48 hours post-injury, symptom resolution, and Return-to-Play and Healthy group at baseline.

\begin{tabular}{|l|l|l|l|l|l|}
\hline & $\mathrm{N}$ & Minimum & Maximum & Mean & Std. Deviation \\
\cline { 2 - 6 } & Statistic & Statistic & Statistic & Statistic & Statistic \\
\hline SysInt_R24hr_avg & 17 & .07 & .23 & .1327 & .04626 \\
SysInt_RSF_avg & 17 & .08 & .24 & .1246 & .03616 \\
SysInt_RRTP_avg & 17 & .07 & .24 & .1266 & .04418 \\
SRG_R24hr_avg & 17 & 1.40 & 6.24 & 3.1349 & 1.51533 \\
SRG_RSF_avg & 17 & 1.33 & 11.83 & 3.5278 & 2.56299 \\
SRG_RRTP_avg & 17 & 1.10 & 9.32 & 2.9942 & 2.01147 \\
PkInt_R24hr_avg & 17 & .06 & .28 & .1857 & .08344 \\
PkInt_RSF_avg & 17 & .04 & .29 & .1821 & .08871 \\
PkInt_RRTP_avg & 17 & .08 & .28 & .1964 & .06801 \\
Aix_R24hr_avg & 17 & .00 & 68.44 & 19.9833 & 18.69202 \\
Aix_RSF_avg & 17 & .00 & 63.56 & 27.7500 & 22.76985 \\
Aix_RRTP_avg & 17 & .00 & 77.16 & 28.6626 & 19.68088 \\
Valid N (listwise) & 17 & & & & \\
\hline
\end{tabular}

\begin{tabular}{|l|l|l|l|l|l|}
\hline & $\mathrm{N}$ & Minimum & Maximum & Mean & Std. Deviation \\
\cline { 2 - 6 } & Statistic & Statistic & Statistic & Statistic & Statistic \\
\hline SysInt_R24hr_avg & 17 & .06 & .24 & .1053 & .03963 \\
SRG_RRTP_avg & 17 & .21 & 7.35 & 3.0354 & 1.75086 \\
PkInt_R24hr_avg & 17 & .01 & .28 & .1915 & .07163 \\
Aix_RRTP_avg & 17 & 7.29 & 70.65 & 27.7236 & 16.88082 \\
Valid N (listwise) & 17 & & & & \\
\hline
\end{tabular}


Table D44. Mann-Whitney U Test - Right Systolic Interval differences between Concussed and Healthy Groups

\section{Ranks}

\begin{tabular}{|ll|l|l|l|}
\hline & Group & N & Mean Rank & Sum of Ranks \\
\hline \multirow{3}{*}{ SysInt_R24hr_avg } & Concussed & 17 & 20.97 & 356.50 \\
& Healthy & 17 & 14.03 & 238.50 \\
& Total & 34 & & \\
\multirow{3}{*}{ SysInt_RSF_avg } & Concussed & 17 & 21.62 & 367.50 \\
& Healthy & 17 & 13.38 & 227.50 \\
& Total & 34 & & \\
& Concussed & 17 & 19.85 & 337.50 \\
SysInt_RRTP_avg & Healthy & 17 & 15.15 & 257.50 \\
& Total & 34 & & \\
\hline
\end{tabular}

Test Statistics $^{\text {a }}$

\begin{tabular}{|l|l|l|l|}
\hline & SysInt_R24hr_avg & SysInt_RSF_avg & SysInt_RRTP_avg \\
\hline Mann-Whitney U & 85.500 & 74.500 & 104.500 \\
Wilcoxon W & 238.500 & 227.500 & 257.500 \\
Z & -2.035 & -2.415 & -1.379 \\
Asymp. Sig. (2-tailed) & .042 & .016 & .168 \\
Exact Sig. [2*(1-tailed Sig.)] & $.041^{\mathrm{b}}$ & $.014^{\mathrm{b}}$ & $.170^{\mathrm{b}}$ \\
\hline
\end{tabular}

a. Grouping Variable: Group

b. Not corrected for ties.

Table D45. Mann-Whitney U Test - Right Steepest Rise Gradient differences between Concussed and Healthy Groups

Ranks

\begin{tabular}{|ll|l|l|l|}
\hline & Group & N & Mean Rank & Sum of Ranks \\
\hline \multirow{3}{*}{ SRG_R24hr_avg } & Concussed & 17 & 17.53 & 298.00 \\
& Healthy & 17 & 17.47 & 297.00 \\
& Total & 34 & & \\
& Concussed & 17 & 18.00 & 306.00 \\
SRG_RSF_avg & Healthy & 17 & 17.00 & 289.00 \\
& Total & 34 & & \\
& Concussed & 17 & 16.88 & 287.00 \\
SRG_RRTP_avg & Healthy & 17 & 18.12 & 308.00 \\
& Total & 34 & & \\
\hline
\end{tabular}


Test Statistics ${ }^{\mathrm{a}}$

\begin{tabular}{|l|l|l|l|}
\hline & SRG_R24hr_avg & SRG_RSF_avg & SRG_RRTP_avg \\
\hline Mann-Whitney U & 144.000 & 136.000 & 134.000 \\
Wilcoxon W & 297.000 & 289.000 & 287.000 \\
Z & -.017 & -.293 & -.362 \\
Asymp. Sig. (2-tailed) & .986 & 770 & .718 \\
Exact Sig. [2*(1-tailed Sig.)] & $1.000^{\mathrm{b}}$ & $.786^{\mathrm{b}}$ & $.734^{\mathrm{b}}$ \\
\hline
\end{tabular}

a. Grouping Variable: Group

b. Not corrected for ties.

Table D46. Mann-Whitney U Test - Right Peak Interval differences between Concussed and Healthy Groups

\begin{tabular}{|ll|l|l|l|}
\multicolumn{5}{l}{ Ranks } \\
\hline & Group & N & Mean Rank & Sum of Ranks \\
\hline \multirow{3}{*}{ PkInt_R24hr_avg } & Concussed & 17 & 17.35 & 295.00 \\
& Healthy & 17 & 17.65 & 300.00 \\
& Total & 34 & & \\
PkInt_RSF_avg & Concussed & 17 & 17.38 & 295.50 \\
& Healthy & 17 & 17.62 & 299.50 \\
& Total & 34 & & \\
PkInt_RRTP_avg & Concussed & 17 & 17.03 & 289.50 \\
& Healthy & 17 & 17.97 & 305.50 \\
& Total & 34 & & \\
\hline
\end{tabular}

Test Statistics ${ }^{\mathbf{a}}$

\begin{tabular}{|l|l|l|l|}
\hline & PkInt_R24hr_avg & PkInt_RSF_avg & PkInt_RRTP_avg \\
\hline Mann-Whitney U & 142.000 & 142.500 & 136.500 \\
Wilcoxon W & 295.000 & 295.500 & 289.500 \\
Z & -.086 & -.069 & -.276 \\
Asymp. Sig. (2-tailed) & .931 & .945 & .783 \\
Exact Sig. [2*(1-tailed Sig.)] & $.946^{\mathrm{b}}$ & $.946^{\mathrm{b}}$ & $.786^{\mathrm{b}}$ \\
\hline
\end{tabular}

a. Grouping Variable: Group

b. Not corrected for ties. 
Table D47. Mann-Whitney U Test - Right Augmentation Index differences between Concussed and Healthy Groups

\begin{tabular}{|ll|l|l|l|}
\hline \multicolumn{5}{c}{ Ranks } \\
\hline & Group & N & Mean Rank & Sum of Ranks \\
\hline \multirow{2}{*}{ Aix_R24hr_avg } & Concussed & 17 & 14.82 & 252.00 \\
& Healthy & 17 & 20.18 & 343.00 \\
& Total & 34 & & \\
& Concussed & 17 & 17.18 & 292.00 \\
Aix_RSF_avg & Healthy & 17 & 17.82 & 303.00 \\
& Total & 34 & & \\
& Concussed & 17 & 17.65 & 300.00 \\
Aix_RRTP_avg & Healthy & 17 & 17.35 & 295.00 \\
& Total & 34 & & \\
\hline
\end{tabular}

Test Statistics ${ }^{\mathrm{a}}$

\begin{tabular}{|l|l|l|l|}
\hline & Aix_R24hr_avg & Aix_RSF_avg & Aix_RRTP_avg \\
\hline Mann-Whitney U & 99.000 & 139.000 & 142.000 \\
Wilcoxon W & 252.000 & 292.000 & 295.000 \\
Z & -1.567 & -.189 & -.086 \\
Asymp. Sig. (2-tailed) & 117 & .850 & .931 \\
Exact Sig. [2*(1-tailed Sig.)] & $.122^{\mathrm{b}}$ & $.865^{\mathrm{b}}$ & $.946^{\mathrm{b}}$ \\
\hline
\end{tabular}

a. Grouping Variable: Group

b. Not corrected for ties.

Table D48. Descriptive statistics for Standardized Assessment of Concussion, Modified Balance Error Scoring System, Total Symptoms, and Symptom Severity for Concussed Group at 24-48 hours post-injury, symptom resolution, and Return-to-Play and for Healthy group at baseline.

\section{Concussed Group}

\begin{tabular}{|l|l|l|l|l|l|}
\hline \multirow{2}{*}{} & $\mathrm{N}$ & Minimum & Maximum & Mean & Std. Deviation \\
\cline { 2 - 6 } & Statistic & Statistic & Statistic & Statistic & Statistic \\
\hline SAC_24hr & 12 & 20.00 & 29.00 & 25.0833 & 3.05877 \\
BESS_24hr & 12 & 20.00 & 29.00 & 25.5000 & 3.00000 \\
TS_24hr & 17 & .00 & 21.00 & 8.9412 & 6.83256 \\
SS_24hr & 17 & .00 & 70.00 & 22.6471 & 20.98494 \\
SAC_SF & 15 & 25.00 & 30.00 & 27.0667 & 1.62422 \\
BESS_SF & 15 & 20.00 & 30.00 & 26.4000 & 2.72029 \\
TS_SF & 15 & .00 & 13.00 & 1.8000 & 3.50917 \\
SS_SF & 15 & .00 & 22.00 & 2.8667 & 6.11633 \\
SAC_RTP & 13 & 24.00 & 30.00 & 28.1538 & 2.19265 \\
BESS_RTP & 13 & 26.00 & 30.00 & 28.3846 & 1.50214 \\
TS_RTP & 14 & .00 & 2.00 & .2857 & .61125 \\
SS_RTP & 14 & .00 & 2.00 & .2857 & .61125 \\
Valid N (listwise) & 7 & & & & \\
\hline
\end{tabular}


Healthy Group

\begin{tabular}{|l|l|l|l|l|l|}
\hline & $\mathrm{N}$ & Minimum & Maximum & Mean & Std. Deviation \\
\cline { 2 - 6 } & Statistic & Statistic & Statistic & Statistic & Statistic \\
\hline SAC_baseline & 17 & 21.00 & 29.00 & 26.4706 & 2.23935 \\
BESS_baseline & 17 & 22.00 & 30.00 & 27.9412 & 2.22122 \\
TS_baseline & 17 & .00 & 9.00 & 1.8235 & 2.57961 \\
SS_baseline & 17 & .00 & 12.00 & 2.5882 & 3.46516 \\
Valid N (listwise) & 17 & & & & \\
\hline
\end{tabular}

Table D49. Mann-Whitney U Test - Standardized Assessment of Concussion differences between Concussed and Healthy Groups

\begin{tabular}{|c|c|c|c|c|}
\hline \multicolumn{5}{|c|}{ Ranks } \\
\hline & Group & $\mathrm{N}$ & Mean Rank & Sum of Ranks \\
\hline \multirow{4}{*}{ SAC_24hr } & Concussed & 12 & 12.63 & 151.50 \\
\hline & Healthy & 17 & 16.68 & 283.50 \\
\hline & Total & 29 & & \\
\hline & Concussed & 15 & 17.40 & 261.00 \\
\hline \multirow[t]{3}{*}{ SAC_SF } & Healthy & 17 & 15.71 & 267.00 \\
\hline & Total & 32 & & \\
\hline & Concussed & 13 & 19.35 & 251.50 \\
\hline \multirow[t]{2}{*}{ SAC_RTP } & Healthy & 17 & 12.56 & 213.50 \\
\hline & Total & 30 & & \\
\hline
\end{tabular}

Test Statistics ${ }^{\text {a }}$

\begin{tabular}{|l|l|l|l|}
\hline & SAC_24hr & SAC_SF & SAC_RTP \\
\hline Mann-Whitney U & 73.500 & 114.000 & 60.500 \\
Wilcoxon W & 151.500 & 267.000 & 213.500 \\
Z & -1.283 & -.517 & -2.121 \\
Asymp. Sig. (2-tailed) & .199 & .605 & .034 \\
Exact Sig. [2*(1-tailed Sig.)] & $211^{\mathrm{b}}$ & $.628^{\mathrm{b}}$ & $.035^{\mathrm{b}}$ \\
\hline
\end{tabular}

a. Grouping Variable: Group

b. Not corrected for ties. 
Table D50. Mann-Whitney U Test - Modified Balance Error Scoring System differences between Concussed and Healthy Groups

\begin{tabular}{|c|c|c|c|c|}
\hline \multicolumn{5}{|c|}{ Ranks } \\
\hline & Group & $\mathrm{N}$ & Mean Rank & Sum of Ranks \\
\hline \multirow{4}{*}{ BESS_24hr } & Concussed & 12 & 10.25 & 123.00 \\
\hline & Healthy & 17 & 18.35 & 312.00 \\
\hline & Total & 29 & & \\
\hline & Concussed & 15 & 13.30 & 199.50 \\
\hline \multirow[t]{3}{*}{ BESS_SF } & Healthy & 17 & 19.32 & 328.50 \\
\hline & Total & 32 & & \\
\hline & Concussed & 13 & 16.04 & 208.50 \\
\hline \multirow[t]{2}{*}{ BESS_RTP } & Healthy & 17 & 15.09 & 256.50 \\
\hline & Total & 30 & & \\
\hline
\end{tabular}

Test Statistics ${ }^{\mathrm{a}}$

\begin{tabular}{|l|l|l|l|}
\hline & BESS_24hr & BESS_SF & BESS_RTP \\
\hline Mann-Whitney U & 45.000 & 79.500 & 103.500 \\
Wilcoxon W & 123.000 & 199.500 & 256.500 \\
Z & -2.553 & -1.833 & -.300 \\
Asymp. Sig. (2-tailed) & .011 & .067 & .764 \\
Exact Sig. [2*(1-tailed Sig.)] & $.011^{\mathrm{b}}$ & $.069^{\mathrm{b}}$ & $.773^{\mathrm{b}}$ \\
\hline
\end{tabular}

a. Grouping Variable: Group

b. Not corrected for ties.

Table D51. Mann-Whitney U Test - Total symptom differences between Concussed and Healthy Groups

\begin{tabular}{|ll|l|l|l|}
\hline \multicolumn{1}{l|l}{ Ranks } \\
\hline \multirow{3}{*}{ TS_24hr } & Group & N & Mean Rank & Sum of Ranks \\
& Concussed & 17 & 23.24 & 395.00 \\
& Healthy & 17 & 11.76 & 200.00 \\
TS_SF & Total & 34 & & \\
& Concussed & 15 & 15.97 & 239.50 \\
& Healthy & 17 & 16.97 & 288.50 \\
TS_RTP & Total & 32 & & 183.50 \\
& Concussed & 14 & 13.11 & 312.50 \\
\hline
\end{tabular}


Test Statistics ${ }^{\mathrm{a}}$

\begin{tabular}{|l|l|l|l|}
\hline & TS_24hr & TS_SF & TS_RTP \\
\hline Mann-Whitney U & 47.000 & 119.500 & 78.500 \\
Wilcoxon W & 200.000 & 239.500 & 183.500 \\
Z & -3.423 & -329 & -1.885 \\
Asymp. Sig. (2-tailed) & .001 & 742 & .059 \\
Exact Sig. [2*(1-tailed Sig.)] & $.000^{\mathrm{b}}$ & $.766^{\mathrm{b}}$ & $109^{\mathrm{b}}$ \\
\hline
\end{tabular}

a. Grouping Variable: Group

b. Not corrected for ties.

Table D52. Mann-Whitney U Test - Symptom Severity differences between Concussed and Healthy Groups

Ranks

\begin{tabular}{|ll|l|l|l|}
\hline & Group & N & Mean Rank & Sum of Ranks \\
\hline \multirow{3}{*}{ SS_24hr } & Concussed & 17 & 23.62 & 401.50 \\
& Healthy & 17 & 11.38 & 193.50 \\
& Total & 34 & & \\
SS_SF & Concussed & 15 & 15.77 & 236.50 \\
& Healthy & 17 & 17.15 & 291.50 \\
& Total & 32 & & \\
SS_RTP & Concussed & 14 & 13.00 & 182.00 \\
& Healthy & 17 & 18.47 & 314.00 \\
\hline
\end{tabular}

Test Statistics ${ }^{\mathrm{a}}$

\begin{tabular}{|l|l|l|l|}
\hline & SS_24hr & SS_SF & SS_RTP \\
\hline Mann-Whitney U & 40.500 & 116.500 & 77.000 \\
Wilcoxon W & 193.500 & 236.500 & 182.000 \\
Z & -3.646 & -.451 & -1.950 \\
Asymp. Sig. (2-tailed) & .000 & .652 & .051 \\
Exact Sig. [2*(1-tailed Sig.)] & $.000^{\mathrm{b}}$ & $.682^{\mathrm{b}}$ & $.100^{\mathrm{b}}$ \\
\hline
\end{tabular}

a. Grouping Variable: Group

b. Not corrected for ties. 
Manuscript III:

Table D53. Descriptive statistics for subject demographics, sport type, and concussion history for concussed and healthy groups.

Concussed Group Descriptive Statistics ${ }^{\mathrm{a}}$

\begin{tabular}{|l|l|l|l|l|l|}
\hline & $\mathrm{N}$ & Minimum & Maximum & Mean & Std. Deviation \\
\hline Age & 16 & 18.00 & 22.00 & 20.0000 & 1.36626 \\
Height & 16 & 149.86 & 195.58 & 175.2600 & 15.18345 \\
Weight & 16 & 46.27 & 150.59 & 85.4454 & 31.52725 \\
Valid N (listwise) & 16 & & & & \\
\hline
\end{tabular}

a. Group $=.00$

\section{Healthy Group Descriptive Statistics ${ }^{\text {a }}$}

\begin{tabular}{|l|l|l|l|l|l|}
\hline & $\mathrm{N}$ & Minimum & Maximum & Mean & Std. Deviation \\
\hline Age & 16 & 18.00 & 21.00 & 19.7500 & .93095 \\
Height & 16 & 157.48 & 198.12 & 177.8000 & 11.91366 \\
Weight & 16 & 54.60 & 133.81 & 82.0901 & 22.25287 \\
Valid N (listwise) & 16 & & & & \\
\hline
\end{tabular}

a. Group $=1.00$

Table D54. Distribution (frequency) of genders, sport type, and number of previous concussion among Concussed Group.

Gender $^{\mathrm{a}}$

\begin{tabular}{|ll|l|l|l|l|}
\hline & & Frequency & Percent & Valid Percent & Cumulative Percent \\
\hline \multirow{4}{*}{ Valid } & .00 & 10 & 62.5 & 62.5 & 62.5 \\
& 1.00 & 6 & 37.5 & 37.5 & 100.0 \\
& Total & 16 & 100.0 & 100.0 & \\
\hline
\end{tabular}

a. Group $=.00$

Sport_type ${ }^{\mathrm{a}}$

\begin{tabular}{|ll|l|l|l|l|}
\hline & & Frequency & Percent & Valid Percent & Cumulative Percent \\
\hline \multirow{4}{*}{ Valid } & 1.00 & 10 & 62.5 & 62.5 & 62.5 \\
& 2.00 & 4 & 25.0 & 25.0 & 87.5 \\
& 3.00 & 2 & 12.5 & 12.5 & 100.0 \\
& Total & 16 & 100.0 & 100.0 & \\
\hline
\end{tabular}

a. Group $=.00$

$\mathrm{CxHx}^{\mathrm{a}}$

\begin{tabular}{|ll|l|l|l|l|}
\hline & & Frequency & Percent & Valid Percent & Cumulative Percent \\
\hline \multirow{4}{*}{ Valid } & .00 & 4 & 25.0 & 25.0 & 25.0 \\
& 1.00 & 12 & 75.0 & 75.0 & 100.0 \\
& Total & 16 & 100.0 & 100.0 & \\
\hline
\end{tabular}

a. Group $=.00$ 


\section{CxNumber $^{\mathrm{a}}$}

\begin{tabular}{|ll|l|l|l|l|}
\hline & & Frequency & Percent & Valid Percent & Cumulative Percent \\
\hline \multirow{4}{*}{ Valid } & .00 & 10 & 62.5 & 62.5 & 62.5 \\
& 1.00 & 3 & 18.8 & 18.8 & 81.3 \\
& 2.00 & 2 & 12.5 & 12.5 & 93.8 \\
& 3.00 & 1 & 6.3 & 6.3 & 100.0 \\
& Total & 16 & 100.0 & 100.0 & \\
\hline
\end{tabular}

a. Group $=.00$

Table D55. Distribution (frequency) of genders, sport type, and number of previous concussion among Concussed Group.

Gender $^{\mathrm{a}}$

\begin{tabular}{|ll|l|l|l|l|}
\hline & & Frequency & Percent & Valid Percent & Cumulative Percent \\
\hline \multirow{4}{*}{ Valid } & .00 & 10 & 56.3 & 56.3 & 56.3 \\
& 1.00 & 6 & 43.8 & 43.8 & 100.0 \\
& Total & 16 & 100.0 & 100.0 & \\
\hline
\end{tabular}

a. Group $=1.00$

Sport_type $^{\mathrm{a}}$

\begin{tabular}{|ll|l|l|l|l|}
\hline & & Frequency & Percent & Valid Percent & Cumulative Percent \\
\hline \multirow{4}{*}{ Valid } & 1.00 & 9 & 56.3 & 56.3 & 56.3 \\
& 2.00 & 7 & 43.8 & 43.8 & 100.0 \\
& Total & 16 & 100.0 & 100.0 & \\
\hline
\end{tabular}

a. Group $=1.00$

$\mathrm{CxHx}^{\mathrm{a}}$

\begin{tabular}{|ll|l|l|l|l|}
\hline & & Frequency & Percent & Valid Percent & Cumulative Percent \\
\hline \multirow{4}{*}{ Valid } & .00 & 13 & 81.3 & 81.3 & 81.3 \\
& 1.00 & 3 & 18.8 & 18.8 & 100.0 \\
& Total & 16 & 100.0 & 100.0 & \\
\hline
\end{tabular}

a. Group $=1.00$

\section{CxNumber $^{a}$}

\begin{tabular}{|ll|l|l|l|l|}
\hline & & Frequency & Percent & Valid Percent & Cumulative Percent \\
\hline \multirow{4}{*}{ Valid } & .00 & 13 & 81.3 & 81.3 & 81.3 \\
& 1.00 & 2 & 12.5 & 12.5 & 93.8 \\
& 3.00 & 1 & 6.3 & 6.3 & 100.0 \\
& Total & 16 & 100.0 & 100.0 & \\
\hline
\end{tabular}

a. Group $=1.00$ 
Table D56. Descriptive statistics for Right and Left Systolic Interval, Steepest Rise Gradient, Peak Interval, and Augmentation Index for Concussed and Healthy Groups.

Concussed Group Descriptive Statistics ${ }^{\mathrm{a}}$

\begin{tabular}{|c|c|c|c|c|c|c|c|c|}
\hline & \multirow[t]{2}{*}{$\mathrm{N}$} & \multirow[t]{2}{*}{ Mean } & \multirow{2}{*}{$\begin{array}{l}\text { Std. } \\
\text { Deviation }\end{array}$} & \multicolumn{5}{|c|}{ Minimum MaximumPercentiles } \\
\hline & & & & & & 25 th & $\begin{array}{l}50 \text { th } \\
\text { (Median) }\end{array}$ & 75 th \\
\hline SysInt_LSF_avg & 16 & .1217 & .04890 & .06 & .23 & .0833 & .1156 & .1365 \\
\hline SRG_LSF_avg & 16 & 3.2394 & 1.57503 & 1.09 & 5.60 & 1.6175 & 3.1218 & 4.7931 \\
\hline PkInt_LSF_avg & 16 & .1792 & .07211 & .06 & .28 & .1172 & .1771 & .2536 \\
\hline Aix_LSF_avg & 16 & 20.6143 & 18.18924 & .00 & 57.04 & 5.9790 & 14.5156 & 39.3735 \\
\hline SysInt_RSF_avg & 16 & .1234 & .03613 & .09 & .24 & 1021 & .1104 & .1365 \\
\hline SRG_RSF_avg & 16 & 3.5674 & 2.59597 & 1.33 & 11.83 & 1.9658 & 2.9587 & 4.2508 \\
\hline PkInt_RSF_avg & 16 & 1986 & .07871 & .06 & .29 & .1130 & .2344 & .2531 \\
\hline Aix_RSF_avg & 16 & 29.9822 & 21.50780 & .00 & 63.56 & 11.1173 & 30.0657 & 50.2006 \\
\hline Group & 16 & .0000 & .00000 & .00 & .00 & .0000 & .0000 & .0000 \\
\hline
\end{tabular}

a. Group $=.00$

Healthy Group Descriptive Statistics ${ }^{\text {a }}$

\begin{tabular}{|c|c|c|c|c|c|c|c|c|}
\hline & \multirow[t]{2}{*}{$\mathrm{N}$} & \multirow[t]{2}{*}{ Mean } & \multirow{2}{*}{$\begin{array}{l}\text { Std. } \\
\text { Deviation }\end{array}$} & \multicolumn{5}{|c|}{ Minimum MaximumPercentiles } \\
\hline & & & & & & 25 th & $\begin{array}{l}50 \text { th } \\
\text { (Median) }\end{array}$ & 75 th \\
\hline SysInt_LSF_avg & 16 & 1070 & .03040 & .06 & .18 & .0854 & .1073 & .1156 \\
\hline SRG_LSF_avg & 16 & 3.8031 & 2.78925 & 1.21 & 10.13 & 1.7600 & 2.8632 & 4.1012 \\
\hline PkInt_LSF_avg & 16 & .1757 & .05837 & .11 & .28 & .1260 & .1552 & .2375 \\
\hline Aix_LSF_avg & 16 & 29.5147 & 21.12440 & 1.04 & 76.98 & 10.2836 & 31.6186 & 44.5405 \\
\hline SysInt_RSF_avg & 16 & .0948 & .01805 & .06 & .13 & .0823 & .0979 & .1078 \\
\hline SRG_RSF_avg & 16 & 3.5617 & 1.49479 & 1.33 & 7.35 & 2.5808 & 3.3663 & 4.1911 \\
\hline PkInt_RSF_avg & 16 & 1949 & .07197 & .01 & .29 & .1474 & .2115 & 2458 \\
\hline Aix_RSF_avg & 16 & 27.2004 & 16.49603 & 7.29 & 64.87 & 12.2806 & 23.6431 & 41.9533 \\
\hline Group & 16 & 1.0000 & .00000 & 1.00 & 1.00 & 1.0000 & 1.0000 & 1.0000 \\
\hline
\end{tabular}

a. Group $=1.00$ 
Table D57. Mann-Whitney U Test - Comparison of Systolic Interval, Steepest Rise Gradient, Peak Interval, and Augmentation Index between Concussed and Healthy Groups.

\section{Ranks}

\begin{tabular}{|c|c|c|c|c|}
\hline & Group & $\mathrm{N}$ & Mean Rank & Sum of Ranks \\
\hline & .00 & 16 & 17.75 & 284.00 \\
\hline \multirow[t]{3}{*}{ SysInt_LSF_avg } & 1.00 & 16 & 15.25 & 244.00 \\
\hline & Total & 32 & & \\
\hline & .00 & 16 & 16.38 & 262.00 \\
\hline \multirow[t]{3}{*}{ SRG_LSF_avg } & 1.00 & 16 & 16.63 & 266.00 \\
\hline & Total & 32 & & \\
\hline & .00 & 16 & 16.31 & 261.00 \\
\hline \multirow[t]{3}{*}{ PkInt_LSF_avg } & 1.00 & 16 & 16.69 & 267.00 \\
\hline & Total & 32 & & \\
\hline & .00 & 16 & 14.81 & 237.00 \\
\hline \multirow[t]{3}{*}{ Aix_LSF_avg } & 1.00 & 16 & 18.19 & 291.00 \\
\hline & Total & 32 & & \\
\hline & .00 & 16 & 21.47 & 343.50 \\
\hline \multirow[t]{3}{*}{ SysInt_RSF_avg } & 1.00 & 16 & 11.53 & 184.50 \\
\hline & Total & 32 & & \\
\hline & .00 & 16 & 15.44 & 247.00 \\
\hline \multirow[t]{3}{*}{ SRG_RSF_avg } & 1.00 & 16 & 17.56 & 281.00 \\
\hline & Total & 32 & & \\
\hline & .00 & 16 & 17.06 & 273.00 \\
\hline \multirow[t]{3}{*}{ PkInt_RSF_avg } & 1.00 & 16 & 15.94 & 255.00 \\
\hline & Total & 32 & & \\
\hline & .00 & 16 & 16.75 & 268.00 \\
\hline \multirow[t]{2}{*}{ Aix_RSF_avg } & 1.00 & 16 & 16.25 & 260.00 \\
\hline & Total & 32 & & \\
\hline
\end{tabular}

Test Statistics

\begin{tabular}{|l|l|l|l|l|}
\hline & SysInt_LSF_avg & SRG_LSF_avg & PkInt_LSF_avg & Aix_LSF_avg \\
\hline Mann-Whitney U & 108.000 & 126.000 & 125.000 & 101.000 \\
Wilcoxon W & 244.000 & 262.000 & 261.000 & 237.000 \\
Z & -.755 & -.075 & -.113 & -1.018 \\
Asymp. Sig. (2-tailed) & .450 & .940 & .910 & .309 \\
Exact Sig. [2*(1-tailed & $.468^{\mathrm{b}}$ & $.956^{\mathrm{b}}$ & $.926^{\mathrm{b}}$ & $.323^{\mathrm{b}}$ \\
Sig.)] & & & & \\
\hline
\end{tabular}

\begin{tabular}{|l|l|l|l|l|}
\hline & SysInt_RSF_avg & SRG_RSF_avg & PkInt_RSF_avg & Aix_RSF_avg \\
\hline Mann-Whitney U & 48.500 & 111.000 & 119.000 & 124.000 \\
Wilcoxon W & 184.500 & 247.000 & 255.000 & 260.000 \\
Z & -3.002 & -.641 & -.339 & -.151 \\
Asymp. Sig. (2-tailed) & .003 & .522 & .734 & .880 \\
Exact Sig. [2*(1-tailed Sig.)] & $.002^{\mathrm{b}}$ & $.539^{\mathrm{b}}$ & $.752^{\mathrm{b}}$ & $.897^{\mathrm{b}}$ \\
\hline
\end{tabular}


Table D58. Descriptive statistics for concussed and healthy group processing speed, complex reaction time, and simple reaction time.

Concussed Group Descriptive Statistics ${ }^{\text {a }}$

\begin{tabular}{|c|c|c|c|c|c|c|c|c|}
\hline & \multirow[t]{2}{*}{$\mathrm{N}$} & \multirow{2}{*}{ Mean } & \multirow{2}{*}{$\begin{array}{l}\text { Std. } \\
\text { Deviation }\end{array}$} & \multirow[t]{2}{*}{ Minimum } & \multicolumn{4}{|c|}{ MaximumPercentiles } \\
\hline & & & & & & 25 th & $\begin{array}{l}50 \text { th } \\
\text { (Median) }\end{array}$ & 75 th \\
\hline CRI_PS & 16 & 2.4613 & .28680 & 1.97 & 2.96 & 2.2225 & 2.4900 & 2.6750 \\
\hline CRI_CRT & 16 & .7625 & .18870 & .59 & 1.31 & .6100 & .7150 & .8275 \\
\hline CRI_SRT & 16 & .4525 & .11498 & .33 & .81 & 3750 & 4250 & 4700 \\
\hline Group & 16 & .0000 & .00000 & .00 & .00 & .0000 & .0000 & .0000 \\
\hline
\end{tabular}

a. Group $=.00$

Healthy Group Descriptive Statistics ${ }^{\text {a }}$

\begin{tabular}{|c|c|c|c|c|c|c|c|c|}
\hline & \multirow[t]{2}{*}{$\mathrm{N}$} & \multirow[t]{2}{*}{ Mean } & \multirow{2}{*}{$\begin{array}{l}\text { Std. } \\
\text { Deviation }\end{array}$} & \multirow[t]{2}{*}{ Minimum } & \multicolumn{4}{|c|}{ MaximumPercentiles } \\
\hline & & & & & & 25 th & $\begin{array}{l}50 \text { th } \\
\text { (Median) }\end{array}$ & 75 th \\
\hline CRI_PS & 16 & 2.6213 & .30316 & 2.11 & 3.27 & 2.3650 & 2.6050 & 2.8150 \\
\hline CRI_CRT & 16 & .7388 & .08437 & .63 & .89 & .6725 & .7150 & .8375 \\
\hline CRI_SRT & 16 & .3900 & .05978 & .31 & .49 & .3300 & .3900 & .4375 \\
\hline Group & 16 & 1.0000 & .00000 & 1.00 & 1.00 & 1.0000 & 1.0000 & 1.0000 \\
\hline
\end{tabular}

a. Group $=1.00$

Table D59. Descriptive statistics for concussed and healthy groups for SAC, BESS, total symptoms, and symptom severity.

Concussed Group Descriptive Statistics ${ }^{\mathbf{a}}$

\begin{tabular}{|c|c|c|c|c|c|c|c|c|}
\hline & \multirow[t]{2}{*}{$\mathrm{N}$} & \multirow[t]{2}{*}{ Mean } & \multirow{2}{*}{$\begin{array}{l}\text { Std. } \\
\text { Deviation }\end{array}$} & \multirow[t]{2}{*}{ Minimum } & \multicolumn{4}{|c|}{ MaximumPercentiles } \\
\hline & & & & & & 25 th & $\begin{array}{l}50 \text { th } \\
\text { (Median) }\end{array}$ & 75 th \\
\hline SAC & 16 & 27.2500 & 1.52753 & 25.00 & 30.00 & 26.0000 & 27.5000 & 28.0000 \\
\hline BESS & 16 & 27.0000 & 2.25093 & 23.00 & 30.00 & 25.0000 & 27.5000 & 29.0000 \\
\hline TotSym & 16 & 4.3125 & 7.58700 & .00 & 22.00 & .0000 & .5000 & 7.5000 \\
\hline SymSev & 16 & 8.2500 & 15.67801 & .00 & 53.00 & .0000 & .5000 & 12.7500 \\
\hline Group & 16 & .0000 & .00000 & .00 & .00 & .0000 & .0000 & .0000 \\
\hline
\end{tabular}

a. Group $=.00$ 
Healthy Group Descriptive Statistics ${ }^{\text {a }}$

\begin{tabular}{|l|l|l|l|l|l|l|l|l|}
\hline & $\mathrm{N}$ & Mean & $\begin{array}{l}\text { Std. } \\
\text { Deviation }\end{array}$ & & Minimum & Maximum & Percentiles & \\
\cline { 7 - 9 } & & & & & & 25 th & $\begin{array}{l}50 \text { th } \\
\text { (Median) }\end{array}$ & 75 th \\
\hline SAC & 16 & 27.1250 & 1.99583 & 23.00 & 30.00 & 26.0000 & 27.0000 & 29.0000 \\
BESS & 16 & 27.3125 & 2.08866 & 22.00 & 29.00 & 26.2500 & 28.0000 & 29.0000 \\
TotSym & 16 & 2.1250 & 2.89540 & .00 & 9.00 & .0000 & .5000 & 3.0000 \\
SymSev & 16 & 3.2500 & 4.10690 & .00 & 12.00 & .0000 & 1.0000 & 6.0000 \\
Group & 16 & 1.0000 & .00000 & 1.00 & 1.00 & 1.0000 & 1.0000 & 1.0000 \\
\hline
\end{tabular}

a. Group $=1.00$

Table D60. Mann-Whitney U Test of Independent Means- Comparison of healthy and concussed group processing speed, complex reaction time, and simple reaction time.

Ranks

\begin{tabular}{|ll|l|l|l|}
\hline & Group & N & Mean Rank & Sum of Ranks \\
\hline \multirow{3}{*}{ CRI_PS } & Concussed & 16 & 14.06 & 225.00 \\
& Healthy & 16 & 18.94 & 303.00 \\
& Total & 32 & & \\
CRI_CRT & Concussed & 16 & 15.78 & 252.50 \\
& Healthy & 16 & 17.22 & 275.50 \\
& Total & 32 & & \\
CRI_SRT & Concussed & 16 & 19.28 & 308.50 \\
& Healthy & 16 & 13.72 & 219.50 \\
& Total & 32 & & \\
\hline
\end{tabular}

Test Statistics

\begin{tabular}{|l|l|l|l|}
\hline & CRI_PS & CRI_CRT & CRI_SRT \\
\hline Mann-Whitney U & 89.000 & 116.500 & 83.500 \\
Wilcoxon W & 225.000 & 252.500 & 219.500 \\
Z & -1.471 & -.434 & -1.681 \\
Asymp. Sig. (2-tailed) & .141 & .664 & .093 \\
Exact Sig. [2*(1-tailed Sig.)] & $149^{\mathrm{b}}$ & $.669^{\mathrm{b}}$ & $.094^{\mathrm{b}}$ \\
\hline
\end{tabular}

a. Grouping Variable: Group

b. Not corrected for ties. 
Table D61. Mann-Whitney U Test of Independent Means- Comparison of healthy and concussed group SAC, BESS, Total Symptoms, and Symptom Severity

Ranks

\begin{tabular}{|ll|l|l|l|}
\hline & Group & N & Mean Rank & Sum of Ranks \\
\hline \multirow{3}{*}{ SAC } & Concussed & 16 & 16.44 & 263.00 \\
& Healthy & 16 & 16.56 & 265.00 \\
& Total & 32 & & \\
BESS & Concussed & 16 & 15.84 & 253.50 \\
& Healthy & 16 & 17.16 & 274.50 \\
& Total & 32 & & \\
TotSym & Concussed & 16 & 16.78 & 268.50 \\
& Healthy & 16 & 16.22 & 259.50 \\
& Total & 32 & & \\
SymSev & Concussed & 16 & 16.59 & 265.50 \\
& Healthy & 16 & 16.41 & 262.50 \\
& Total & 32 & & \\
\hline
\end{tabular}

Test Statistics ${ }^{\mathrm{a}}$

\begin{tabular}{|l|l|l|l|l|}
\hline & SAC & BESS & TotSym & SymSev \\
\hline Mann-Whitney U & 127.000 & 117.500 & 123.500 & 126.500 \\
Wilcoxon W & 263.000 & 253.500 & 259.500 & 262.500 \\
Z & .038 & -.403 & -.182 & -.060 \\
Asymp. Sig. (2-tailed) & .969 & .687 & .856 & .952 \\
Exact Sig. [2*(1-tailed Sig.)] & $.985^{\mathrm{b}}$ & $.696^{\mathrm{b}}$ & $.867^{\mathrm{b}}$ & $.956^{\mathrm{b}}$ \\
\hline
\end{tabular}

a. Grouping Variable: Group

b. Not corrected for ties. 
Table D62. Spearman Rho correlations between CRI and BAM variables.

\begin{tabular}{|c|c|c|c|c|c|}
\hline & & & CRI_PS & CRI_CRT & CRI_SRT \\
\hline \multirow{18}{*}{ Spearman's rho } & SysInt_LSF_avg & $\begin{array}{l}\text { Correlation } \\
\text { Coefficient } \\
\text { Sig. (2-tailed) } \\
\text { N }\end{array}$ & $\begin{array}{l}033 \\
857 \\
32\end{array}$ & $\mid \begin{array}{l}327 \\
067 \\
32\end{array}$ & $\begin{array}{l}-.049 \\
788 \\
32\end{array}$ \\
\hline & SRG_LSF_avg & $\begin{array}{l}\text { Correlation } \\
\text { Coefficient } \\
\text { Sig. (2-tailed) } \\
\text { N }\end{array}$ & $\begin{array}{l}238 \\
189 \\
32\end{array}$ & $\begin{array}{l}227 \\
212 \\
32\end{array}$ & $\begin{array}{l}.068 \\
711 \\
32\end{array}$ \\
\hline & PkInt_LSF_avg & $\begin{array}{l}\text { Correlation } \\
\text { Coefficient } \\
\text { Sig. (2-tailed) } \\
\text { N }\end{array}$ & $\begin{array}{l}.381^{*} \\
.032 \\
32\end{array}$ & $\begin{array}{l}241 \\
184 \\
32\end{array}$ & $\begin{array}{l}-.007 \\
.969 \\
32\end{array}$ \\
\hline & \multirow{3}{*}{ Aix_LSF_avg } & $\begin{array}{l}\text { Correlation } \\
\text { Coefficient }\end{array}$ & .335 & .119 & -.002 \\
\hline & & Sig. (2-tailed) & .061 & .515 & .990 \\
\hline & & $\mathrm{N}$ & 32 & 32 & 32 \\
\hline & \multirow{3}{*}{ SysInt_RSF_avg } & $\begin{array}{l}\text { Correlation } \\
\text { Coefficient }\end{array}$ & -.091 & .127 & .320 \\
\hline & & Sig. (2-tailed) & .620 & 489 & .074 \\
\hline & & $\mathrm{N}$ & 32 & 32 & 32 \\
\hline & \multirow{3}{*}{ SRG_RSF_avg } & $\begin{array}{l}\text { Correlation } \\
\text { Coefficient }\end{array}$ & .181 & .178 & -.069 \\
\hline & & Sig. (2-tailed) & .321 & 331 & .709 \\
\hline & & $\mathrm{N}$ & 32 & 32 & 32 \\
\hline & \multirow{3}{*}{ PkInt_RSF_avg } & $\begin{array}{l}\text { Correlation } \\
\text { Coefficient }\end{array}$ & .349 & .205 & -.069 \\
\hline & & Sig. (2-tailed) & .050 & .260 & .709 \\
\hline & & $\mathrm{N}$ & 32 & 32 & 32 \\
\hline & \multirow{3}{*}{ Aix_RSF_avg } & $\begin{array}{l}\text { Correlation } \\
\text { Coefficient }\end{array}$ & .334 & .181 & -.103 \\
\hline & & Sig. (2-tailed) & .061 & .322 & .573 \\
\hline & & $\mathrm{N}$ & 32 & 32 & 32 \\
\hline
\end{tabular}


Table D63. Spearman Rho correlations between SAC, BESS, Total Symptoms, and Symptom Severity and BAM variables.

\begin{tabular}{|c|c|c|c|c|c|c|}
\hline & & & SAC & BESS & TotSym & SymSev \\
\hline \multirow{24}{*}{ Spearman's rho } & \multirow{3}{*}{ SysInt_LSF_avg } & Correlation Coefficient & -.297 & -.216 & -.021 & -.013 \\
\hline & & Sig. (2-tailed) & .099 & .235 & .908 & .945 \\
\hline & & $\mathrm{N}$ & 32 & 32 & 32 & 32 \\
\hline & \multirow{3}{*}{ SRG_LSF_avg } & Correlation Coefficient & .063 & -.043 & -.095 & -.081 \\
\hline & & Sig. (2-tailed) & .733 & .814 & .603 & .658 \\
\hline & & $\mathrm{N}$ & 32 & 32 & 32 & 32 \\
\hline & \multirow{3}{*}{ PkInt_LSF_avg } & Correlation Coefficient & -.028 & -.194 & .181 & .193 \\
\hline & & Sig. (2-tailed) & .879 & .287 & 322 & .291 \\
\hline & & $\mathrm{N}$ & 32 & 32 & 32 & 32 \\
\hline & \multirow{3}{*}{ Aix_LSF_avg } & Correlation Coefficient & .009 & -.107 & .180 & .197 \\
\hline & & Sig. (2-tailed) & .962 & .560 & 323 & .281 \\
\hline & & $\mathrm{N}$ & 32 & 32 & 32 & 32 \\
\hline & \multirow{3}{*}{ SysInt_RSF_avg } & Correlation Coefficient & -.050 & -.104 & .001 & -.012 \\
\hline & & Sig. (2-tailed) & .785 & .570 & .995 & .948 \\
\hline & & $\mathrm{N}$ & 32 & 32 & 32 & 32 \\
\hline & \multirow{3}{*}{ SRG_RSF_avg } & Correlation Coefficient & -.053 & .085 & -.253 & -.260 \\
\hline & & Sig. (2-tailed) & .775 & .645 & .163 & .151 \\
\hline & & $\mathrm{N}$ & 32 & 32 & 32 & 32 \\
\hline & \multirow{3}{*}{ PkInt_RSF_avg } & Correlation Coefficient & -.158 & .029 & .102 & .093 \\
\hline & & Sig. (2-tailed) & .387 & .875 & .577 & 613 \\
\hline & & $\mathrm{N}$ & 32 & 32 & 32 & 32 \\
\hline & \multirow{3}{*}{ Aix_RSF_avg } & Correlation Coefficient & -.012 & -.164 & -.034 & -.031 \\
\hline & & Sig. (2-tailed) & .948 & .368 & .852 & .865 \\
\hline & & $\mathrm{N}$ & 32 & 32 & 32 & 32 \\
\hline
\end{tabular}


Table D64. Step-wise Linear Regression Model - Prediction of Processing Speed by left and right augmentation index and peak interval.

\section{Model Summary}

\begin{tabular}{|c|c|c|c|c|c|c|c|c|c|}
\hline \multirow[t]{2}{*}{ Model } & \multirow[t]{2}{*}{$\mathrm{R}$} & \multirow{2}{*}{$\begin{array}{l}\mathrm{R} \\
\text { Square }\end{array}$} & \multirow{2}{*}{$\begin{array}{l}\text { Adjusted R } \\
\text { Square }\end{array}$} & \multirow{2}{*}{$\begin{array}{l}\text { Std. Error } \\
\text { of the } \\
\text { Estimate }\end{array}$} & \multicolumn{5}{|c|}{ Change Statistics } \\
\hline & & & & & $\begin{array}{l}\text { R Square } \\
\text { Change }\end{array}$ & \begin{tabular}{|l} 
F \\
Change
\end{tabular} & df1 & df2 & $\begin{array}{l}\text { Sig. F } \\
\text { Change }\end{array}$ \\
\hline 1 & $383^{\mathrm{a}}$ & .147 & .118 & .28309 & .147 & 5.154 & 1 & 30 & .031 \\
\hline
\end{tabular}

a. Predictors: (Constant), PkInt_LSF_avg

Coefficients $^{\mathrm{a}}$

\begin{tabular}{|ll|l|l|l|l|l|}
\hline \multirow{2}{*}{ Model } & \multicolumn{2}{|l|}{ Unstandardized Coefficients } & $\begin{array}{l}\text { Standardized } \\
\text { Coefficients }\end{array}$ & & Sig. \\
\cline { 2 - 5 } & & B & Std. Error & Beta & & \\
\hline \multirow{2}{*}{1} & (Constant) & 2.224 & .148 & & 14.986 & .000 \\
& PkInt_LSF_avg & 1.788 & .788 & .383 & 2.270 & .031 \\
\hline
\end{tabular}

a. Dependent Variable: CRI_PS

Excluded Variables ${ }^{a}$

\begin{tabular}{|ll|l|l|l|l|l|}
\hline Model & & Beta In & $\mathrm{t}$ & Sig. & $\begin{array}{l}\text { Partial } \\
\text { Correlation }\end{array}$ & $\begin{array}{l}\text { Collinearity } \\
\text { Statistics }\end{array}$ \\
\cline { 5 - 7 } & & & & & Tolerance \\
\hline \multirow{2}{*}{1} & Aix_LSF_avg & $.146^{\mathrm{b}}$ & .661 & .514 & .122 & .591 \\
& PkInt_RSF_avg & $.119^{\mathrm{b}}$ & .513 & .612 & .095 & .542 \\
& Aix_RSF_avg & $.149^{\mathrm{b}}$ & .675 & .505 & .124 & .596 \\
\hline
\end{tabular}

a. Dependent Variable: CRI_PS

b. Predictors in the Model: (Constant), PkInt_LSF_avg

$\underline{\text { Table D65. Descriptive statistics for time to assessment for concussed group. }}$

Descriptive Statistics ${ }^{\mathbf{a}}$

\begin{tabular}{|c|c|c|c|c|c|c|c|c|c|}
\hline & $\mathrm{N}$ & \multicolumn{3}{|c|}{ Minimum|Maximum|Mean } & Std. & \multicolumn{2}{|c|}{ Skewness } & \multicolumn{2}{|c|}{ Kurtosis } \\
\hline & Statistic & Statistic & Statistic & Statistic & Statistic & Statisti & $\begin{array}{l}\text { Std. } \\
\text { Error }\end{array}$ & Statisti & $\begin{array}{l}\text { Std. } \\
\text { Error }\end{array}$ \\
\hline $\begin{array}{l}\text { time_of_assessment } \\
\text { Valid N (listwise) }\end{array}$ & $\begin{array}{l}16 \\
16\end{array}$ & 48.00 & 288.00 & 108.0000 & 78.87205 & 1.269 & .564 & .258 & 1.091 \\
\hline
\end{tabular}

a. Group = Concussed 
APPENDIX E

Back Matter 


\author{
Executive Summary \\ Exercise \& Sport Injury Laboratory \\ University of Virginia
}

Project Title: $\quad$ Assessment of cerebral blood flow changes following sport related concussion in collegiate and high school athletes.

Project Supervisor: Susan Saliba, PhD, ATC, MPT, FNATA (Assistant Professor of Kinesiology)

Research Team: Shellie Acocello, MSEd, ATC (PhD student in Sports Medicine) Donna Broshek, $\mathrm{PhD}$ (Director of Department of Pyschiatry and Neurobehavioral Sciences) Joseph Hart, PhD, ATC (Assistant Professor of Kinesiology) Jay Hertel, PhD, ATC, FNATA, FACSM (Professor of Kinesiology)

Purpose: (1) To determine the reliability of arterial stiffness measures obtained using the Brain Acoustic Monitor. (2) To determine if concussed and healthy individuals differ on measures of arterial stiffness obtained by the Brain Acoustic Monitor. (3) To determine if differences in arterial stiffness predict neurocognitive test scores.

\title{
Research questions:
}

\section{Manuscript I:}

- Are the outcomes associated with the Brain Acoustic Monitor consistent in dayto-day assessments?

- Are the outcomes obtained using the Brain Acoustic Monitor repeatable between different clinicians?

\section{Manuscript II:}

- Do measures of arterial stiffness including systolic interval, steepest rise gradient, peak interval, and augmentation index obtained using the Brain Acoustic Monitor differ in concussed individuals 24 to 48 hours post-injury, at resolution of symptoms, and return-to-activity?

- Do concussed individuals and healthy age, gender, and sport contact matched controls differ in systolic interval, steepest rise gradient, peak interval, and augmentation index at important clinical time points following injury?

\section{Manuscript III:}


- Do concussed individuals and healthy, age, gender, and sport contact matched controls differ in systolic interval, steepest rise gradient, peak interval, and augmentation index at resolution of symptoms?

- Are deficits in neurocognitive function correlated with measures of arterial stiffness in healthy and concussed individuals?

- Do any measures of arterial stiffness predict poorer neurocognitive test scores in healthy and concussed individuals?

Subjects: 22 concussed individuals and 22 age, gender, and sport-matched healthy controls.

\section{Inclusion criteria:}

\section{Manuscript 1:}

- Healthy, recreationally active individuals (3 x 20 min per week minimum)

- No history of concussion or other head injury during the previous six months

- No presence of neurological disorder that would affect balance or cognition

- No history of seizures

\section{Manuscript II \& III:}

Healthy control group

- Participants in Division I or interscholastic athletics at UVA, VMI, Longwood, or St. Anne's Belfield School

- Availability of baseline neurocognitive test scores

Concussed group:

- Diagnosis of concussion by athletic trainer or team physician

- Availability of baseline and post-injury neurocognitive test scores

\section{Exclusion criteria:}

\section{Manuscript I:}

- History of concussion or other head injury during previous 6 months

- Presence of neurological disorder that would affect balance or cognition

- History of seizures

\section{Manuscript II \& III:}

Both groups:

$\circ$ No presence of neurological disorder that would affect balance or cognition

- No history of seizures

Healthy group only:

$\circ$ History of concussion or other head injury during previous 6 months 


\section{Study design:}

Manuscript I: repeated measures laboratory study

Manuscript II: case-control, repeated measures

Manuscript II: case-control, descriptive

\section{Independent Variables:}

\section{Manuscript I:}

1. Time: day 1 vs day 2

2. Assessor: assessor 1 vs. assessor 2

\section{Manuscript II:}

1. Time (concussed group only): initial assessment, symptom resolution, return-toplay

2. Group: concussed vs. healthy

\section{Manuscript III:}

1. Group: concussed vs. healthy

\section{Dependent Variables:}

\section{Manuscript I:}

1. BAM variables: Systolic interval, Steepest rise gradient, Peak Interval, Augmentation Index

\section{Manuscript II:}

1. BAM variables: systolic interval, steepest rise gradient, peak interval, augmentation index

2. SCAT3 outcomes: SAC total score, BESS errors, Total Symptoms, Symptom Severity

3. Concussed only: time to symptom resolution, time to return-to-play

Manuscript III:

1. BAM variables: systolic interval, steepest rise gradient, peak interval, augmentation index

2. SCAT3 outcomes: SAC total score, BESS errors, Total Symptoms, Symptom Severity

3. Processing speed, complex reaction time, and simple reaction time z-scores

Procedures:

Manuscript I: 
1. Subjects reviewed for eligibility and consent form signed.

2. Patient demographics and exercise history forms filled out.

3. BAM measures obtained every 15 minutes for 1 hour ( $0 \mathrm{~min}, 15 \mathrm{~min}, 30 \mathrm{~min}, 45$ $\mathrm{min}, 60 \mathrm{~min}$ ). For a subset of 10 subjects, two assessors obtained measurements at each interval.

4. Subjects return $>48$ hours for repeat of BAM measures every 15 minutes for 1 hour (0 min, $15 \mathrm{~min}, 30 \mathrm{~min}, 45 \mathrm{~min}, 60 \mathrm{~min}$ ).

\section{Manuscripts II \& III:}

1. Subjects recruited. For concussed subjects, athletic trainers notified study investigators within 24 hours of injury.

2. Eligibility reviewed and consent form signed.

3. Patient demographics and concussion history forms filled out.

4. SCAT3 administered by certified athletic trainer.

5. BAM measures obtained.

a. For concussed individuals, BAM and SCAT3 was repeated at symptom resolution and upon clearance by team athletic trainer or physician for return-to-activity. (i.e. steps $4 \& 5$ are performed at least 3 times for all concussed individuals).

b. For healthy individuals, BAM and SCAT3 testing was performed prior to first game of season (i.e. during preseason).

6. Assessment using computerized neurocognitive testing battery performed.

a. For all subjects, a baseline assessment was performed prior to preseason practices.

b. For concussed subjects, post-injury neurocognitive assessment occurred at resolution of symptoms as a pre-requisite for initiation of a graded returnto-play protocol.

Significance of the study: Clinical assessment of concussion currently relies on measures of the functional manifestations of the metabolic and molecular dysfunction that occurs following injury. While this method has been useful and a multifactorial approach is necessary, there is a need for more objective tools for determination of true recovery. If we are able to better define recovery from concussion, it is likely the long term consequences of concussion, such as early onset dementia or chronic traumatic encephalopathy, may be avoided or their severity reduced. If the BAM is proven as a useful tool in the assessment of concussion, sports medicine professionals would finally have a direct method of assessing a physiological consequence of concussion and returnto-activity guidelines could be made more effective in reducing re-injury and later life cognitive dysfunction. 


\section{Recommendations for Future Research}

1. Inclusion of baseline data for comparison to post-injury arterial stiffness waveforms

2. Validation of BAM measures as measures of arterial function by inducing changes in systemic arterial blood pressure and/or alterations in the partial pressure of arterial carbon dioxide

3. Stratification of waveform types based on anatomical characteristics of anterior cerebral anatomy

4. Comparison to other measures of cerebral blood flow including advanced neuroimaging, SPECT, NIRS, and/or transcranial Doppler ultrasound. 


\section{BIBLIOGRAPHY}

1. Gardner A, Iverson GL, McCrory P. Chronic traumatic epncephalopathy in sport: A systematic review. Br J Sports Med. 2013;48:84 - 90.

2. Mez J, Stern RA, McKee AC. Chronic traumatic encephalopathy: Where are we and where are we going? Curr Neurol Neurosci Rep. 2013;13:407.

3. Langlois JAs, Rutland-Brown W, Wald MA. The epidemiology and impact of traumatic brain injury: A brief overview. Journal of Head Trauma Rehabilitation. 2006;21(5):375-376-378.

4. McCrory P, Meeuwisse WH, Aubry M, et al. Consensus statement on concussion in sport: The 4th international conference on concussion in sport held in zurich, november 2012. British journal of sports medicine. 2013;47:250.

5. Guskiewicz KM, Bruce SL, Cantu RC, et al. National athletic trainers' association position statement: Management of sport-related concussion. Journal of athletic training. 2004;9(3):280-281-297.

6. Gall B, Parkhouse W, Goodman D. Heart rate variability of recently concussed athletes at rest and exercise. Med Sci Sports Exerc. 2004;36(8):1269-1274.

7. Dutton RP, Prior K, Cohen R, et al. Diagnosing mild traumatic brain injury: Where are we now? Journal of Trauma Injury, Infection, and Critical Care. 2011;70(3):554-555-559.

8. Dutton RP, Sewell J, Aarabi B, Scalea TM. Preliminary trial of a noninvasive brain acoustic monitor in trauma patients with severe closed head injury. Journal of Trauma. 2002;53:857-858-863.

9. LaMonte MP, Sewell J, Bahouth MN, Sewell C. A noninvasive portable acoustic diagnostic system to differentiate ischemic from hemorrhagic stroke. $J$ Neuroimaging. 2005; 15:57.

10. Acocello S, Broshek DK, Saliba S. Inter-rater and intra-rater reliability of cerebral blood flow measures obtained using the brain acoustic monitor. $J$ Neurosci Methods. 2014;221:85-91.

11. Harmon KG, Drezner JA, Gammons M, et al. American medical society for sports medicine position statement: Concussion in sport. Br J Sports Med. 2013;47:15-26.

12. National Center for Injury Prevention and Control. Report to congress on mild traumatic brain injury in the united states: Steps to prevent a serious public health problem. Atlanta, GA: Centers for Disease Control and Prevention; 2003:56.

13. Shultz MR, Marshall SW, Mueller FO, et al. Incidence and risk factors for concussion in high school athletes, north carolina, 1996-1999. Am J Epidemiol. 2004;160(10):937-944.

14. Collins MW, Iverson GL, Lovell MR, McKeag DB, Norwig J, Maroon J. On-field predictors of neuropsychological and symptom deficit following sports-related concussion. Clin J Sports Med. 2003;13(4):222-229.

15. Covassin T, Swanik CB, Sachs ML. Sex differences and the incidence of concussions among collegiate athletes. J Athl Train. 2003;38:238-244. 
16. Powell JW, Barber-Foss KD. Traumatic brain injury in high school athletes. JAMA. 1999;282:958-963.

17. Mansell J, Tierney RT, Sitler MR, Swanik KA, Stearne D. Resistance training and head-neck segment dynamic stabilization in male and female collegiate soccer players. J Athl Train. 2005;40:310-319.

18. Queen RM, Weinhold PS, Kirkendall DT, Yu B. Theoretical study of the effect of ball properties on impact force in soccer heading. Med Sci Sports Exerc. 2003;35:2069-2076.

19. Tierney RT, Sitler MR, Swanik CB, Swanik KA, Higgins M, Torg J. Gender differences in head-neck segment dynamic stabilization during head acceleration. Med Sci Sports Exerc. 2005;37:272-279.

20. Vertinsky PA. The eternally wounded woman: Women, doctors, and exercise in the late nineteeth century. Urbana, IL: University of Illinois Press; 1994.

21. Gessel LM, Fields SK, Collins CL, Dick RW, Comstock RD. Concussions among united states high school and collegiate athletes. J Athl Train. 2007;42(4):495-503.

22. Guskiewicz KM, Weaver NL, Padua DA, Garrett WE. Epidemiology of concussion in collegiate and high school football players. Am J Sports Med. 2000;28:643-650.

23. Guskiewicz KM, Mihalik JP. Biomechanics of sport concussion: Quest for the elusive injury threshold. Exerc Sport Scie Rev. 2011;39(1):4.

24. Castile L, Collins CL, McIlvain NM, Comstock RD. The epidemiology of new versus recurrent sports concussions among high school athletes, 2005 - 2010. Br J Sports Med. 2011

25. Marar M, McIlvain NM, Fields SK, Comstock RD. Epidemiology of concussions among united states high school athletes in 20 sports. A Journal Sports Med. 2012

26. Register-Mihalik JK, Guskiewicz KM, Valovich McLeod TC, Linnan LA, Mueller FO, Marshall SW. Knowledge, attitude, and concussion-reporting behaviors among high school athletes: A preliminary study. J Athl Train. 2013;48(5):645-653.

27. Ommaya AK, Gennarelli TA. Cerebral concussion and traumatic unconsciousness: Correlation of experimental and clinical observations of blunt head injuries. Brain. 1974;97(4):633-654.

28. Mihalik JP, Blackburn JT, Greenwald RM, Cantu RC, Marshall SW, Guskiewicz KM. Collision type and player anticipation affect head impact severity among youth ice hockey players. Pediatrics. 2010;125:e1394-e1401.

29. Mihalik JP, Guskiewicz KM, Jeffries JA, Greenwald RM, Marshall SW. Characteristics of head impacts sustained by youth ice hockey players. Journal of Sports Engineering and Technology. 2008;222:45.

30. Mihalik JP, Bell DR, Marshall SW, Guskiewicz KM. Measurement of head impacts in collegiate football players: An investigation of positional and event-type differences. Neurosurgery. 2007;61:1229-1235.

31. Guskiewicz KM, McCrea M, Marshall SW, et al. Cumulative effects associated with recurrent concussion in collegiate football players: The NCAA concussion study. JAMA. 2003;290:2549-2555. 
32. Schnebel B, Gwin JT, Anderson S, Gatlin R. In vivo study of head impacts in football: A comparison of national collegiate athletic association division I versus high school impacts. Neurosurgery. 2007;60:490-496.

33. Guskiewicz KM, Mihalik JP, Shankar V, et al. Measurement of head impacts in colleigate football players - relationship between head impact biomechanics and acute clinical outcome after concussion. Neurosurgery. 2007;61:1244-1253.

34. Duhaime AC, Beckwith JG, Maerlender AC, et al. Spectrum of acute clinical characteristics of diagnosed concussions in college athletes wearing instrumented helmets. J Neurosurg. 2012;117(6):1092.

35. Viano DC, Pellman EJ. Concussion in professional football: Biomechanics of the striking player. Neurosurgery. 2005;56(2):266 - 280.

36. Dezman ZDW, Ledet EH, Kerr HA. Neck strength imbalance correlates with increased head acceleration in soccer heading. 2013. Sports Healthy: A Multidisciplinary Approach;5(4):320.

37. Barkhoudarian G, Hovda DA, Giza CC. The molecular pathophysiology of concussive brain injury. Clin Sports Med. 2011;30:33-48.

38. Faden AI, Demediuk P, Panter SS, et al. The role of excitatory amino acids and NMDA receptors in traumatic brain injury. Science. 1989;244(4906):798-800.

39. Katayama Y, Becker DP, Tamura T, et al. Massive increases in extracellular potassium and the indiscriminate release of glutamate following concussive brain injury. J Neurosurg. 1990;73(6):889-900.

40. Hovda DA, Yoshino A, Kawamata T, et al. Diffuse prolonged depression of cerebral oxidase histochemistry study. Brain Res. 1991;567(1):1-10.

41. Kalimo H, Rehncrona S, Soderfeldt B. The role of lactic acidosis in the ischemic nerve cell injury. Acta Neuropathol Suppl. 1981;7:20-22.

42. Vagnozzi R, Signoretti S, Cristofori L, et al. Assessment of metabolic brain damage and recovery following mild traumatic brain injury: A multicentre, proton magnetic resonance spectroscopic study in concussed patients. Brain. 2010:1.

43. Vagnozzi R, Signoretti S, Tavazzi B. Temporal window of metabolic brain vulnerability to concussion: A pilot $1 \mathrm{H}$-magnetic resonance spectroscopic study in concussed athletes--part III. Neurosurgery. 2008;62(6):1286-1295.

44. Lynn R, Abou-Chebl A. Cerebral arterial anatomy. In: Macdonald S, Stansby G, eds. Practical carotid artery stenting. London: Springer-Verlag; 2009:63. 10.1007/978-1-84800-299-9_6.

45. Krishnaswamy A, Klein JP, Kapadia SR. Clinical cerebrovascular anatomy. Catherization and Cardiovascular Interventions. 2010;75:530-539.

46. Hendrikse J, van Raamt AF, van der Graaf Y, Mali W, van der Grond J. Distribution of cerebral blod flow in the circle of willis. Radiology. 2005;235:185189.

47. Len TK, Neary JP. Cerebrovascular pathophysiology following mild traumatic brain injury. Clin Physiol Funct Imaging. 2011;31:85-93.

48. Pittman AL, Ping JS, Mark JB. Arterial and central venous pressure monitoring. International Anaesthesiology. 2004;42(1):13-30. 
49. Peterson EC, Wang Z, Britz G. Regulation of cerebral blood flow. International Journal of Vascular Medicine. 2011:1.

50. O'Rourke MF, Safar ME. Relationship between aortic stiffening and microvascular disease in brain and kidney. Hypertension. 2005;46:200.

51. Rangel-Castilla L, Gasco J, Nauta HJ, Okonkwo DO, Robertson CS. Cerebral pressure autoregulation in traumatic brain injury. Neurosurg Focus. 2008;25:E7.

52. Akca O. Optimizing the intraoperative management of carbon dioxide concentration. Curr Opin Anasethesiol. 2006;19:19-25.

53. Ainslie P, Duffin J. Integration of cerebrovascular $\mathrm{CO} 2$ reactivity and chemoreflex control of breathing: Mechanisms of regulation, measurement, and interpretation. Am J Physiol Regul Integr Comp Physiol. 2009;296:R1473-R1495.

54. Len TK, Neary P, Asmundson GJG, Goodman DG, Bjornson B, Bhambhani YN. Cerebrovascular reactivity impairment after sports-induced concussion. Medicine \& Science in Sport \& Exercise. 2011:2241.

55. Tegeler C, Kim J, Collins G, et al. Dynamic vascular assessment of brain circulation for sports-related concussion. European Society of Neurosonology and Cerebral Hemodynamics, Wetzlar, Germany. 2004:1-38.

56. Becelewski J, Pierzchala K. Cerebrovascular reactivity in patients with mild head injury. Neurol Neurochir Pol. 2003;37:339.

57. Junger EC, Ghatan S, Douville CM, Lam AM, Aaslid R, Winn HR. Cerebral autoregulation following minor head injury. N Neurosurg. 1997;86:425-432.

58. McQuire JC, Sutcliffe JC, Coats TJ. Early changes in middle cerebral artery blood flow velocity after head injury. J Neurosurg. 1998;89:526-532.

59. Golding EM, Steenberg CF, Krishnappa I, Robertson CS, Bryan RM. Cerebrovascular reactivity to $\mathrm{CO} 2$ and hypotension after mild cortical impact injury. Am J Physiol Heart Circ Physiol. 1999;277:H1457.

60. Giza CC, Hovda DA. The neurometabolic cascade of concussion. J Athl Train. 2001;36:228-235.

61. Bonne O, Gilboa A, Louzoun Y, et al. Cerebral blood flow in chronic symptomatic mild traumatic brain injury. Pyschiatry Research: Neuroimaging. 2003;124:141.

62. Grindel SH. Epidemiology and pathophysiology of minor traumatic brain injury. Curr Sports Med Rep. 2003;2:18-23.

63. Werner C, Engelhard K. Pathophysiology of traumatic brain injury. Br J Anaesthesia. 2007;1:4.

64. Strebel S, Lam AM, Matta BF, Newell DW. Impaired cerebral autoregulation after mild brain injury. Surg Neurol. 1997;47:128.

65. Schramm P, Klein KU, Pape M, et al. Serial measurement of static and dynamic cerebrovascular autoregulation after brain injury. J Neurosurg Anesthesiol. 2011;23(1):41-44.

66. Golding EM, Robertson CS, Bryan RM. The consequences of traumatic brain injury on cerebral blood flow and autoregulation: A review. Clin Exp Hypertens. 1999;21:299.

67. Maugans TA, Farley C, Altaye M, Leach J, Cecil KM. Pediatric sports-related concussion produces cerebral blood flow alterations. Pediatrics. 2012;129:28. 
68. Ge Y, Patel MB, Chen Q, et al. Assessment of thalamic perfusion in patients with mild traumatic brain injury by true FISP arterial spin labelling MR imaging at 3T. Brain Injury. 2009;23(7-8):666-674.

69. Cote C, Neary P, Goodman D, Parkhouse W, Bhambhani YN. Cerebral oxygenation and blood volume changes during exercise following sport-induced concussion (abstract). Appl Physiol Nutr Metab. 2006;31 (Suppl):S14.

70. Chen JK, Johnston KM, Frey S, Petrides M, Worsley K, Ptito A. Functional abnormalities in symptomatic concussed athletes: An fMRI study. Neuroimage. 2004;22:68-82.

71. Davis GA, Iverson GL, Guskiewicz KM, Ptito A, Johnston KM. Contributions of neuroimaging, balance testing, electrophysiology, and blood markers to the assessment of sport-related concussion. Br J Sports Med. 2009;43 (Suppl):i36-i45.

72. Jantzen KJ, Anderson B, Steinberg FL, Kelso JA. A prospective functional MR imaging study of mild traumatic brain injury in college football players. AJNR Am J Neuroradiol. 2004;25:738-745.

73. Lovell MR, Pardini JE, Welling J, et al. Functional brain abnormalities are related to clinical recovery and time to return-to-play. Neurosurgery. 2007;61:352-360.

74. Dutton RP, Van der Heijden MS, Aarabi B, Sewell J, Scalea TM. Screening TBI patients with the brain acoustic monitor: Association with CT scan findings and neurologic status at hospital discharge. Clinical Intensive Care. 2005;16(2):97-98105.

75. Laurent S, Cockcroft J, Van Bortel L, et al. Expert consensus document on arterial stiffness: Methodological issues and clinical applications. European Heart Journal. 2006;27:2588.

76. Poels MMF, van Oijen M, Mattace-Raso FUS, et al. Arterial stiffness, cognitive decline, and risk of dementia: The rotterdam study. Stroke. 2007;38:888.

77. Pase MP, Pipingas A, Kras M, et al. Healthy middle-aged individuals are vulnerable to cognitive deficits as a result of increased arterial stiffness. $J$ Hypertension. 2010;28:1724.

78. Waldstein SR, Rice SC, Thayer JF, Najjar SS, Scuteri A, Zonderman AB. Pulse pressure and pulse wave velocity are related to cognitive decline in the baltimore longitudinal study of aging. Hypertension. 2008;51:99.

79. McCrory P, Johnston K, Meeuwisse WH, et al. Summary and agreement statement of the 2nd international conference on concussion in sport, prague 2004. Br J Sports Med. 2005;39:196-204.

80. McCrory P, Meeuwise W, Johnston K, et al. Consensus statement on concussion in sport - the 3rd international conference on concussion in sport held in zurich, november 2008. Clin J Sports Med. 2009;19(3):185-200.

81. Barr WB, Prichep LS, Chabot R, et al. Measuring brain electrical activity to track recovery from sport-related concussion. Brain Inj. 2012;26:58-66.

82. McCrea M, Prichep LS, Powell MR, et al. Acute effects and recovery after sportrelated concussion: A neurocognitive and quantitative brain electrical activity study. J Head Trauma Rehabil. 2010;25:283-292. 
83. McCrea M, Guskiewicz KM, Randolph C, et al. Effects of a symptom-free waiting period on clinical outcome and risk of reinjury after sport-related concussion. Neurosurgery. 2009;65:876-882.

84. McCrea M, Kelly JP, Randolph C, et al. Immediate neurocognitive effects of concussion. Neurosurgery. 2002;50:1032-1040.

85. Daniel JC, Nassiri JD, Wilckens J, et al. The implementation and use of the standardized assessment of concussion at the U.S. naval academy. Mil Med. 2002;167:873-876.

86. Jinguji TM, Bompadre V, Harmon KG, et al. Sport concussion assessment tool - 2: Baseline values for high school athletes. Br J Sports Med. 2012;46:365.

87. Valovich McLeod TC, Bay RC, Lam KC, Chhabra A. Representative baseline values on the sport concussion assessment tool 2 (SCAT2) in adolescent athletes vary by gender, grade, and concussion history. Am J Sports Med. 2012;XX(XXX):1.

88. Guskiewicz K, Perrin DH, Gansneder BM. Effect of mild head injury on postural control stability in athletes. J Athl Train. 1996;31(4):300-306.

89. Guskiewicz KM, Riemann BL, Perrin DH, et al. Alternative approaches to the assessment of mild head injury in athletes. Med Sci Sports Exerc. 1997;29(Suppl 7):S213-S221.

90. Mrazik M, Ferrara MS, Peterson CL, et al. Injury severity and neuropsychological and balance outcomes of four college athletes. Brain Inj. 2000;14(10):921-931.

91. Peterson CL, Ferrara MS, Mrazik M, et al. Evaluation of neuropsychological domain scores and postural stability following cerebral concussion in sports. Clin J Sports Med. 2003;13:230-237.

92. Riemann BL, Guskiewicz KM. Effects of mild head injury on postural stability as measured through clinical balance testing. J Athl Train. 2000;35:19-25.

93. Guskiewicz KM, Ross SE, Marshall SW. Postural stability and neuropsychological deficits after concussion in collegiate athletes. J Athl Train. 2001;36:263-273.

94. Guskiewicz KM. Balance assessment in the management of sport-related concussion. Clin J Sports Med. 2011;30:89-102.

95. McCrea M, Guskiewicz KM, Marshall SW, et al. Acute effects and recovery time following concussion in collegiate football players: The NCAA concussion study. JAMA. 2003;290:2556-2563.

96. McCrea M, Barr WB, Guskiewicz K, et al. Standard regression-based methods for measuring recovery after sport-related concussion. J Int Neuropsychol Soc. 2005;11:58-69.

97. Riemann BL, Guskiewicz KM, Shields EW. Relationship between clinical and force plate measures of postural stability. J Sport Rehabil. 1999;8:71-82.

98. Bressel E, Yonker JC, Kras J, et al. Comparison of static and dynamic balance in female collegiate soccer, basketball, and gymnastics athletes. J Athl Train. 2007;42:42-46.

99. Docherty CL, Valovich-McLeod TC, Shultz SJ. Postural control deficits in participants with functional ankle instability as measured by the balance error scoring system. Clin J Sports Med. 2006;16:203-208. 
100. Susco TM, Valovich-McLeod TC, Gansneder BM, et al. Balance recovers within 20 minutes after exertion as measured by the balance error scoring system. $J$ Athl Train. 2004(39):241-246.

101. Wilkins JC, Valovich-McLeod TC, Perrin DH, et al. Performance on the balance error scoring system decreases after fatigue. J Athl Train. 2004;39:156-61.

102. Valovich McLeod TC, Perrin DH, Guskiewicz KM, et al. Serial administration of clinical concussion assessments and learning effects in healthy young athletes. Clin J Sports Med. 2004;14:287-295.

103. Valovich TC, Perrin DH, Gansneder BM. Repeat administration elicits a practice effect with the balance error scoring system but not with the standardized assessment of concussion in high school athletes. J Athl Train. 2003;38:51-56.

104. Erlanger D, Saliba E, Barth J, Almquist J, Webright W, Freeman J. Monitoring resolution of postconcussion symptoms in athletes: Preliminary results of a webbased neuropsychological test protocol. J Athl Train. 2001;36(3):280-287.

105. Iverson GL, Lovell MR, Collins MW. Validiy of ImPACT for measuring processing speed following sports-related concussion. Journal of Clinical and Experimental Neuropsychology. 2005;27:683-689.

106. Iverson GL, Lovell M, Collins MW. Interpreting change in ImPACT following sport concussion. Clin Neuropsychol. 2003;17:460.

107. Allen BJ, Gfeller JD. The immediate post-concussion assessment and cognitive testing battery and traditional neuropsychological measures: A construct and concurrent validity study. Brain Injury. 2011;25(2):179-191.

108. Schatz P, Pardini JE, Lovell MR, Collins MW, Podell K. Sensitivity and specificity of the ImPACT test battery for concussion in athletes. Arch Clin Neuropsychol. 2006;21:91-99.

109. Schatz P, Sandel N. Sensitivity and specificity of the online version of the ImPACT in high school and collegiate athletes. Am J Sports Med. 2012;41(2):321-326.

110. Broglio SP, Macciocchi SN, Ferrara MS. Sensitivity of the concussion assessment battery. Neurosurgery. 2007;60:1050-1058.

111. Lovell MR, Collins MW, Iverson GL, et al. Recovery from mild concussion in high school athletes. J Neurosurg. 2003;98:296-301. 